\title{
The intestinal barrier function : an integrative approach to understanding gastrointestinal pathophysiology
}

Citation for published version (APA):

Keszthelyi, D. (2014). The intestinal barrier function : an integrative approach to understanding gastrointestinal pathophysiology. [Doctoral Thesis, Maastricht University]. Maastricht University. https://doi.org/10.26481/dis.20141023dk

Document status and date:

Published: 01/01/2014

DOI:

10.26481/dis.20141023dk

Document Version:

Publisher's PDF, also known as Version of record

Please check the document version of this publication:

- A submitted manuscript is the version of the article upon submission and before peer-review. There can be important differences between the submitted version and the official published version of record.

People interested in the research are advised to contact the author for the final version of the publication, or visit the DOI to the publisher's website.

- The final author version and the galley proof are versions of the publication after peer review.

- The final published version features the final layout of the paper including the volume, issue and page numbers.

Link to publication

\footnotetext{
General rights rights.

- You may freely distribute the URL identifying the publication in the public portal. please follow below link for the End User Agreement:

www.umlib.nl/taverne-license

Take down policy

If you believe that this document breaches copyright please contact us at:

repository@maastrichtuniversity.nl

providing details and we will investigate your claim.
}

Copyright and moral rights for the publications made accessible in the public portal are retained by the authors and/or other copyright owners and it is a condition of accessing publications that users recognise and abide by the legal requirements associated with these

- Users may download and print one copy of any publication from the public portal for the purpose of private study or research.

- You may not further distribute the material or use it for any profit-making activity or commercial gain

If the publication is distributed under the terms of Article $25 \mathrm{fa}$ of the Dutch Copyright Act, indicated by the "Taverne" license above, 
The intestinal barrier function: an integrative approach to understanding gastrointestinal pathophysiology 
๑ Copyright Daniel Keszthelyi, Maastricht 2014

ISBN: 978-94-6259-325-1

Coverdesign and Layout: Khaya Ludidi

Cover image from: Andreas Vesalius, De humani corporis fabrica, Basel 1555

Printing: Ipskamp Drukkers, Enschede

The studies presented in this thesis were performed within the framework of TI Food and Nutrition and within NUTRIM School for Nutrition, Toxicology and Metabolism, which participates in the Graduate School VLAG (Food Technology. Agrobiotechnology, Nutrition and Health Sciences), accredited by the Royal Netherlands Academy of Arts and Sciences.

Printing of this thesis was financially supported by: Nederlandse Vereniging voor Gastroenterologie, de Sectie Experimentele Gastroenterologie van de NVGE, ABN AMRO, Will Pharma, Grünenthal, academisch ziekenhuis Maastricht, Ferring, Dr Falk Benelux, Abbvie. 


\section{The intestinal barrier function: an integrative approach to understanding gastrointestinal pathophysiology}

\section{Proefschrift}

ter verkrijging van de graad van doctor aan de Universiteit Maastricht, op gezag van de Rector Magnificus, Prof. dr. L.L.G. Soete volgens het besluit van het College van Decanen, in het openbaar te verdedigen op donderdag 23 oktober 2014 om 14.00 uur

door

Daniel Keszthelyi 


\section{Promotor}

Prof. dr. A.A.M. Masclee

Copromotor

Dr. F.J. Troost

\section{Beoordelingscommissie}

Prof. dr. A.M.W.J. Schols, voorzitter

Prof. dr. Q. Aziz, Queen Mary University of London

Prof. dr. C.H.C. Dejong

Prof. dr. W.J. de Jonge, Academisch Medisch Centrum, Amsterdam

Prof. dr. H.W.M. Steinbusch 
To my grandparents 


\section{TABLE OF CONTENTS}

Part I. Intestinal barrier function: Role of tryptophan metabolites

10 Chapter 1

30 Chapter 2

56 Chapter 3

74 Chapter 4

Part II. Visceral hypersensitivity in IBS: methods and mechanisms 88 Chapter 5

112 Chapter 6

156 Chapter 7

Part III. The putative relationship between intestinal barrier function, serotonin metabolism and visceral hypersensitivity in IBS

176 Chapter 8

206 Chapter 9

development

234 Chapter 10

246 Chapter 11

264 Chapter 12

286 Chapter 13 
Introduction

Tryptophan metabolites

Acute tryptophan depletion

Kynurenic acid in IBS

Mechanisms of visceral hypersensitivity

Methods to assess visceral hypersensitivity

TRPV1 and neuropeptides in pain generation

Mechanistic insight using the serotonin precursor 5-

hydroxytryptophan

Systematic review on the role of drugs in microscopic colitis

Drug exposure in microscopic colitis

Drug exposure in IBS

Discussion

Appendices 
D. Keszthelyi

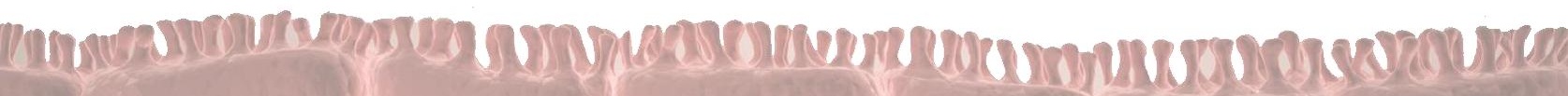




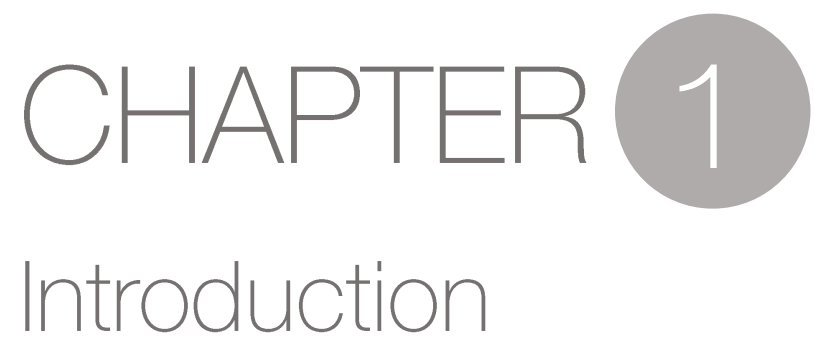

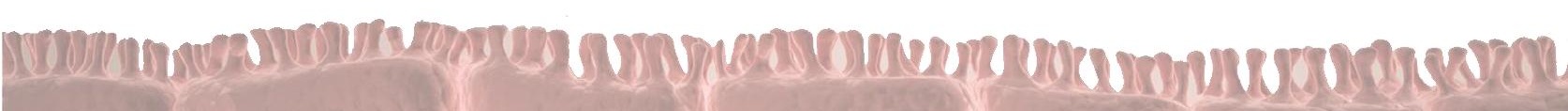




\subsection{GENERAL INTRODUCTION}

The intestinal epithelium is by far the most extensive mucosal surface in the human body that provides an interface between the external environment and the host. Thousands of components derived from ingested food as well as microorganisms transiently or permanently present in the gastrointestinal tract interact continuously with the intestinal epithelium. This condition requires a unique function that enables distinction between substances that are beneficial to the organism (such as nutrients and commensal microbiota), and those that are potentially noxious or toxic (such as pathogenic bacteria). This selective intestinal barrier function ensures homeostatic control of not only the gastrointestinal tract, but is also of importance to systemic function.

The intestinal barrier has several components. First, there is luminal degradation of bacteria and antigens by gastric acid and pancreatic juice; in addition, commensal intestinal bacteria inhibit the colonization of pathogens by production of antimicrobial substances and by competing for nutrients that are required for pathogen growth. Second, the microclimate close to the epithelium consisting of the unstirred water layer, glycocalyx, and mucus layer prevent bacterial adhesion and contain antimicrobial products secreted by Paneth cells and secretory IgA. Third, below the unstirred water layer, glycocalyx, and mucus layer, epithelial cells connected to each other by tight junctions form a physical barrier. ${ }^{1,2}$

Another important component of the intestinal barrier, the immunological barrier, is located subepithelially in the lamina propria. The lamina propria of the intestinal mucosa comprises a large number of immune cells with potent effector functions. Cells of the innate immune system, such as macrophages and mast cells, are instrumental in limiting the extent of invasion by luminal microbes and producing chemokines and cytokines to attract different populations of leucocytes. Effector mechanisms of the adaptive immune system complement these responses by quantitative and qualitative regulation of the local immune response. Approximately $70 \%$ of all the lymphocytes of the human body are concentrated in the intestinal intra-epithelial and subepithelial layers, and the largest 
pool of tissue macrophages is located in the intestinal wall. ${ }^{3}$ Therefore, intestinal immunity must maintain the delicate balance between the capacity for mounting protective immune responses to infectious agents, and the ability to tolerate (or ignore) the luxuriant load of antigens present in the intestinal lumen. ${ }^{4}$ It is also important to note that the immunological barrier is able to maintain mucosal homeostasis in case of epithelial barrier dysfunction or even complete loss of the barrier, but some data suggest that the epithelium orchestrates these immunoregulatory events through direct interactions with innate immune cells. ${ }^{2}$ This again highlights a cardinal role for the intestinal epithelium in regulating homeostasis.

This thesis primarily focuses on the investigation of intestinal epithelium, which, as mentioned previously, constitutes a key element of the intestinal barrier. It has the unique ability to sense and recognize luminal compounds and thereby initiates physiological responses responsibly for orchestrating gastrointestinal function. The intestinal epithelial cells form an adjacent monolayer, which also serves as a selective barrier. This function is established through the formation of complex protein-protein networks that mechanically link adjacent cells and seal the intercellular space. These consist of tight junctions, adherens junctions and desmosomes. Tight junctions are located closest to the lumen and are composed of transmembrane proteins (occludins, claudins) and plaque proteins (ZO protein family, among others) and are associated with the intracellular actinmyosin cytoskeleton. ${ }^{2}$ Nutrients, such as glucose and amino acids are able to induce opening of the tight junctions and increase paracellular permeability, which facilitates absorption of nutrients (see also below). This opening is regulated through a series of signal transductory pathways all resulting in the increased activity of myosin light chain kinase, which phosphorylates myosin and causes a contraction of cytoskeletal components and conformational changes in structures associated with it, such as the tight junctions. Hence, this dynamic process of the opening and closing of the tight junction complex regulates the paracellular transport of luminal substances into the lamina propria. ${ }^{2}$ 


\subsection{PHYSIOLOGICAL REGULATION OF TIGHT JUNCTION}

Regulation of tight junction function is therefore crucial in determining the passage of certain molecules from the intestinal lumen, which communicates with the external environment, to the lamina propria of the intestine, which in this sense ensures entrance via the intestine to the systemic compartment and other areas or organs of the human body connected to the systemic compartment.

The regulation of the gastrointestinal uptake of glucose provides an elegant example of tight junction function. Glucose can be taken up transcellularly by active co-transport with sodium through the sodium-glucose linked transporter (SGLT-1). Accumulation of sodium intracellularly can in turn activate the sodium-hydrogen exchanger 3 (NHE3) on the apical membrane of epithelial cells. Experimental evidence has shown that activation of NHE3 results in phosphorylation of the myosin light chain, leading to actinomyosin condensation and eventually opening of the tight junctions. ${ }^{5}$ This allows, simultaneously to active transcellular transport, the passive paracellular uptake of glucose. This mechanism becomes especially relevant when the luminal concentration of glucose is higher, resulting in saturation of the active transporter. In a physiological sense, opening of the tight junctions in such way facilitates the rapid postprandial uptake of glucose from the lumen.6, 7

\subsection{IMPAIRED INTESTINAL BARRIER FUNCTION}

Dysregulation of the intestinal barrier function, for instance through malfunctioning of the tight junctions, may in theory lead to exposure of the internal milieu to potentially noxious substances. Such exposure can in turn result in an initiation of an immune reaction of the host inducing inflammation, disease development or propagation.?

Interestingly, it appears that molecules involved in the physiological regulation of tight junction function, such as the myosin light chain kinase, are also known to have an important role in intestinal barrier dysfunction under pathological conditions. For instance, 
it has been demonstrated that inflammatory cytokines, such as tumor necrosis factor (TNF)-alpha and interferon (IFN)-gamma can induce the activity of myosin light chain kinase, resulting in increased intestinal permeability.8, 9 This mechanism has been clearly demonstrated to play a role in inflammatory bowel disease (IBD). 10, 11

In the past two decades, we have witnessed an increased appreciation and knowledge of the dysfunction of the intestinal barrier as being an important pathogenetic mechanism and key factor not only in gastrointestinal, but also systemic diseases. Increased gut permeability is associated with several different human diseases, including IBD, celiac disease, and irritable bowel syndrome (IBS), but also diabetes mellitus, allergy and asthma. ${ }^{2}$ However, whether this increase in gut permeability is an epiphenomenon, an early manifestation of disease, or a critical step in disease pathogenesis remains unknown and is still under debate.

\subsection{THE ROLE OF THE INTESTINAL BARRIER IN IRRITABLE BOWEL SYNDROME}

Of particular interest is the role of the intestinal barrier function in irritable bowel disease (IBS). IBS is a common gastrointestinal disorder, affecting $10-15 \%$ of the population in developed countries. ${ }^{13}$ Often referred to as a functional gastrointestinal disorder, it is characterized by lower abdominal discomfort or pain with disturbed defecation in the absence of apparent structural or biochemical abnormalities that might explain the symptoms. ${ }^{13}$ According to current hypotheses, an increase in intestinal permeability in the IBS patients may lead to entrance of noxious substances into the lamina propria, which can, on one hand, excite mucosal nerve endings contributing to pain symptom generation, and on the other hand can initiate an immune reaction resulting in mucosal inflammation. ${ }^{14,15}$ Such mucosal inflammation can hence increase intestinal permeability, for instance through action of inflammatory cytokines, creating a vicious circle of impaired barrier function. 
Figure shows an integrative overview of the current pathophysiological concept of IBS.

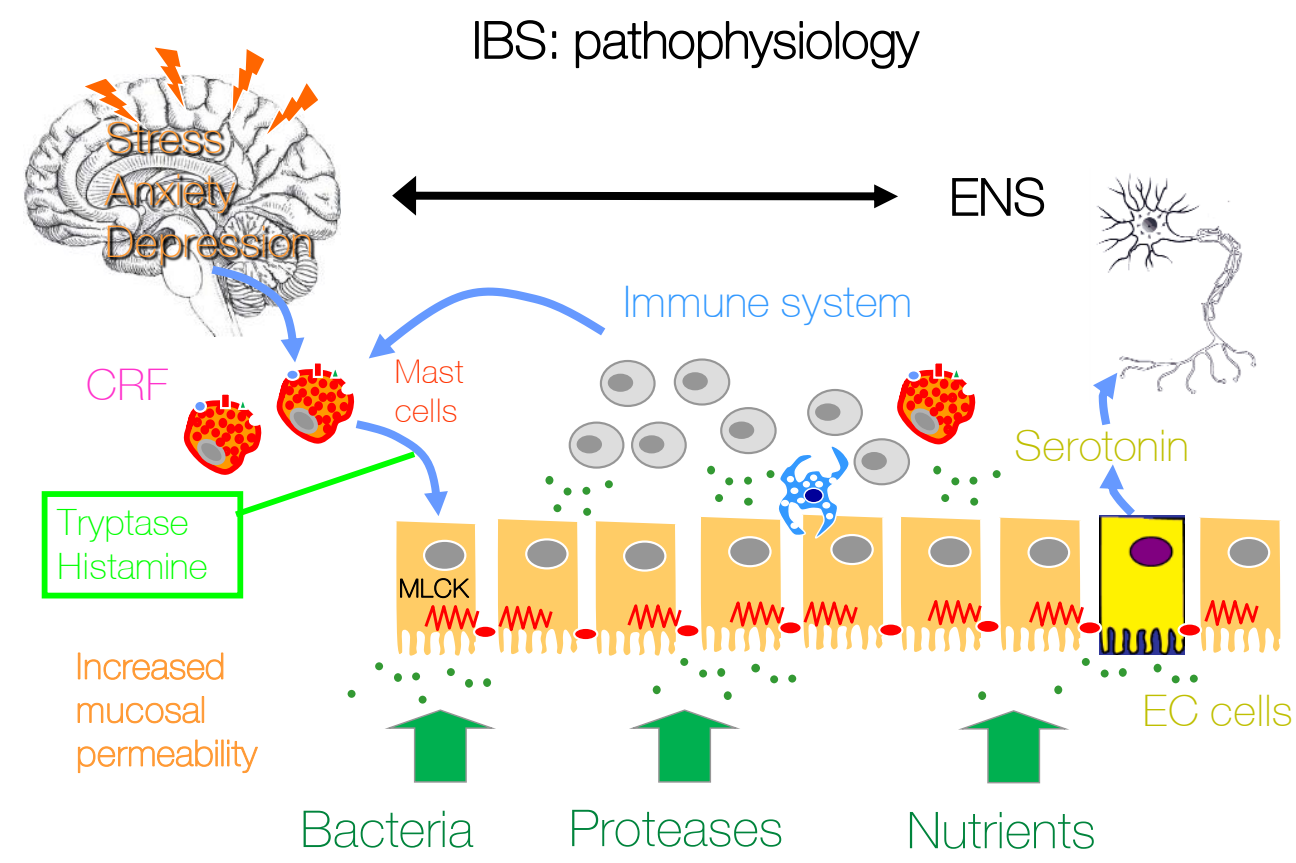

Figure 1: Schematic diagram of pathophysiological mechanisms in IBS.

Important to note are the following components of this pathophysiological concept:

> Numerous luminal agents (bacteria, ${ }^{16}$ proteases $^{17}$ and nutrients ${ }^{18}$ ) can influence the intestinal barrier.

> An increase in permeability can result in interaction of luminal content with immune cells and mast cells in the lamina propria, resulting in immune activation. ${ }^{19}$

> Luminal agents, including food-derived substances, can activate enterochromaffin cells, a group of specialized epithelial cells, which serve as sensory transducers by releasing serotonin upon activation that in turn can activate nervous cells of the enteric nervous system and visceral afferents projecting to the central nervous system through activating serotonin receptors. ${ }^{20}$

> The central nervous system has been also shown to influence intestinal barrier 
function. Stress, anxiety and depression, often-observed comorbid conditions in IBS, can lead to increased release of CRF, which can activate mast cells in the intestinal mucosa, resulting in tryptase and histamine release. ${ }^{21}$ These substances are believed to affect intestinal barrier function and mucosal immune responses and can also contribute to excitation of visceral afferents. ${ }^{14}$ On the other hand, extrinsic vagal and/or sympathetic efferents or enteric nerves influence the mucosal barrier through direct effects via acetylcholine or vasoactive intestinal polypeptide on epithelial cells, tight junction protein expression, or through interaction with immune cells. ${ }^{1}$ These mechanisms illustrate the important bidirectional communication between the central nervous system and the intestine, commonly referred to as the gut-brain axis.

The factors described above briefly illustrate the complexity and redundancy of mechanisms involved in the pathogenesis of IBS. However, there appears to be a common denominator, namely an impaired intestinal barrier function, which is the major focus and subject of this thesis.

With respect to alterations of the intestinal barrier function, the following key triggers have been identified: food, microbial changes (infection and dysbiosis), toxins (including drugs) and stress. Perturbation of the intestinal barrier results in exposure of sensory afferent endings to luminal content, which can lead to sustained sensitization of afferents contributing to visceral hyperalgesia and development of pain symptoms characteristic for IBS. ${ }^{14}$ We are particularly interested in the role of serotonin in relation to this hypothesis, considering the involvement of this mediator in sensory function of the intestinal epithelium. We believe that investigation of the serotonergic metabolism and signaling in terms of the regulation of the intestinal barrier may increase our understanding of the pathophysiology of IBS, and in particular increased visceral perception.

In this thesis, key aim is to explore the interrelationship between intestinal barrier function, serotonergic metabolism and visceral hypersensitivity. We specifically aimed to investigate this interrelationship by modulating serotonergic metabolism through a 


\section{Chapter 1}

nutritional type of intervention (see Figure 2).

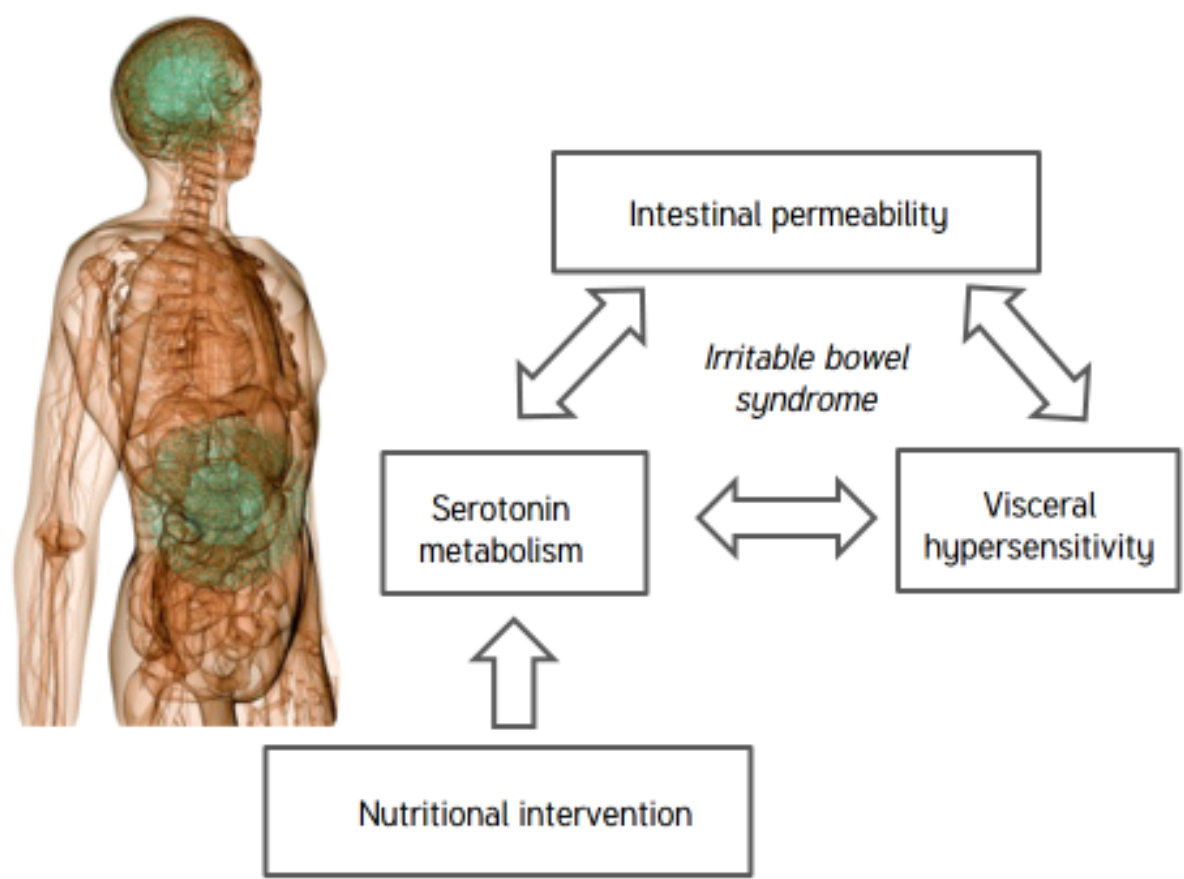

Figure 2: Schematic diagram of the interrelationship of visceral perception, intestinal barrier function and serotonergic metabolism.

\subsection{MICROSCOPIC COLITIS: BARRIER DEFECT LEADING TO OVERT INFLAMMATION}

Microscopic colitis (MC) is increasingly recognized as a cause of chronic watery diarrhea and abdominal pain. In fact, $M C$ is diagnosed in up to $13 \%$ of patients investigated for chronic diarrhea, particularly if middle-aged or elderly. ${ }^{22}$ The annual incidence of around 5 per 100,000 inhabitants.22, 23 It is characterized by macroscopically normal ileocolonoscopy and typical microscopic findings of increased number of intraepithelial CD8+ T-lymphocytes, accompanied by superficial epithelial damage and a variable inflammatory infiltrate in the lamina propria. In addition, in collagenous colitis, a subtype 
of MC, a thickened subepithelial collagen band, which exceeds $10 \mu \mathrm{m}$, is present. ${ }^{24}$ The symptoms of diarrhea-predominant IBS are similar to microscopic colitis, such that it is not possible to differentiate these entities in the clinical setting without colon biopsies. A recent prospective study in fact revealed that 38 to $58 \%$ of patients with $M C$ fulfilled the criteria for IBS. ${ }^{25}$

More interestingly, it appears that MC and IBS may share a common pathophysiological background, i.e. an impairment in the intestinal barrier function. ${ }^{26}$ In fact, some consider IBS and MC to represent two different entities among a wide spectrum of chronic intestinal inflammatory conditions, with active IBD at the outermost end of this spectrum. ${ }^{27}$ According to this point of view, increased permeability results in mucosal inflammation, which can be absent or low-grade (IBS), microscopically detectable (MC) or macroscopically present (acute inflammatory colitis).

With respect to the pathogenesis of $\mathrm{MC}$, our attention has been drawn to the role of exposure to certain drugs prior to the development of symptoms. ${ }^{28} \mathrm{~A}$ remarkable number of case reports have been published in which drug exposure has been described as a clear cause for the development of microscopic colitis. In particular exposure to NSAIDs, which are known to impair intestinal barrier function, have been found to be associated with MC. ${ }^{29}$ This underlies the importance of assessing drug use in not only patients with $\mathrm{MC}$, but also in patients with IBS, considering the overlap in symptoms and potentially also common or shared pathogenetic background.

\subsection{METHODOLOGICAL ASPECTS: MEASUREMENT OF INTESTINAL BARRIER FUNCTION}

\section{IN VIVO MEASUREMENT OF INTESTINAL PERMEABILITY}

Assessment of the permeability of the paracellular pathway in vivo can be performed using simple, noninvasive means relying on the oral administration small probes, such as sugars. Traditionally, these small molecules are inert, water-soluble substances that are 


\section{Chapter 1}

taken up by the intestine and are secreted in unchanged form in urine, where they can be readily detected. Thus far, intestinal permeability has been measured by urinary excretion of two probes of different sizes but similar transit and uptake processes, calculating the excretion ratio of a monosaccharide and a disaccharide such as mannitol and lactulose, respectively. These probes differ in manner of transport, i.e. paracellular or transcellular. In this way, two routes of uptake are compared. The most widely accepted method of measuring intestinal permeability in the small intestine is the lactulose/mannitol or lactulose/rhamnose urine excretion test in humans. In the healthy small bowel, the permeability for larger sugars such as lactulose is much lower than for smaller sugars such as mannitol or rhamnose. Lactulose and other larger molecules pass through the intercellular spaces, which are regulated by intercellular tight junctions. Under pathological conditions (such as mucosal inflammation) the permeability for the larger sugars increases, whereas the permeability of the smaller sugars remains stable or decreases. This results in an increased urinary excretion ratio of large to small sugars. ${ }^{30}$, 31

Lactulose and rhamnose are subject to bacterial degradation in the colon. The ratio of lactulose and rhamnose is therefore believed to reflect permeability of the small intestine. Sucralose and erythritol, on the other hand, are not subject to bacterial degradation and therefore can be used to determine whole-gut permeability. ${ }^{32}$ While these sugars when used for permeability tests are measured in urine, plasma concentrations may also reflect passage through the intestinal barrier and thereby reflect barrier function without any interference with renal function or hydration state. In this thesis we focus on measurements of plasma concentrations instead of urinary concentrations of these sugars. We anticipate that plasma measurements reflect more accurately acutely occurring changes in intestinal permeability.

\section{TIGHT UUNCTION PROTEIN TRANSCRIPTION AND EXPRESSION}

Biopsy specimens are also suitable to assess transcription and expression of different tight junction proteins. Isolation of mRNA and subsequent qPCR allows quantification of 
tight junction transcription. Immunohistochemical staining of tight junction proteins allows not only the determination of the absolute amount of protein, but also the assessment of the intracellular distribution of the different tight junction proteins.

\subsection{AMM AND OUTLINE OF THE THESIS}

In this thesis, we aimed to investigate in detail the intestinal barrier function, with specific attention for its potential role in the pathogenesis of disease. We here focused on two gastrointestinal disorders: irritable bowel syndrome and microscopic colitis. The following main core subjects were investigated:

> The potential role of tryptophan metabolites, in particular serotonin, in the regulation of the intestinal barrier.

> The role of intestinal barrier dysfunction in the development of visceral hypersensitivity, considered a 'hallmark' of IBS.

> The interrelationship between intestinal barrier function, serotonergic metabolism and visceral perception in relation to the pathogenesis of IBS.

> The potential role of drug exposure in the development of IBS and microscopic colitis by virtue of alteration of the intestinal barrier function. 


\section{Chapter 1}

The thesis has been therefore divided into four parts (see Figure 3).

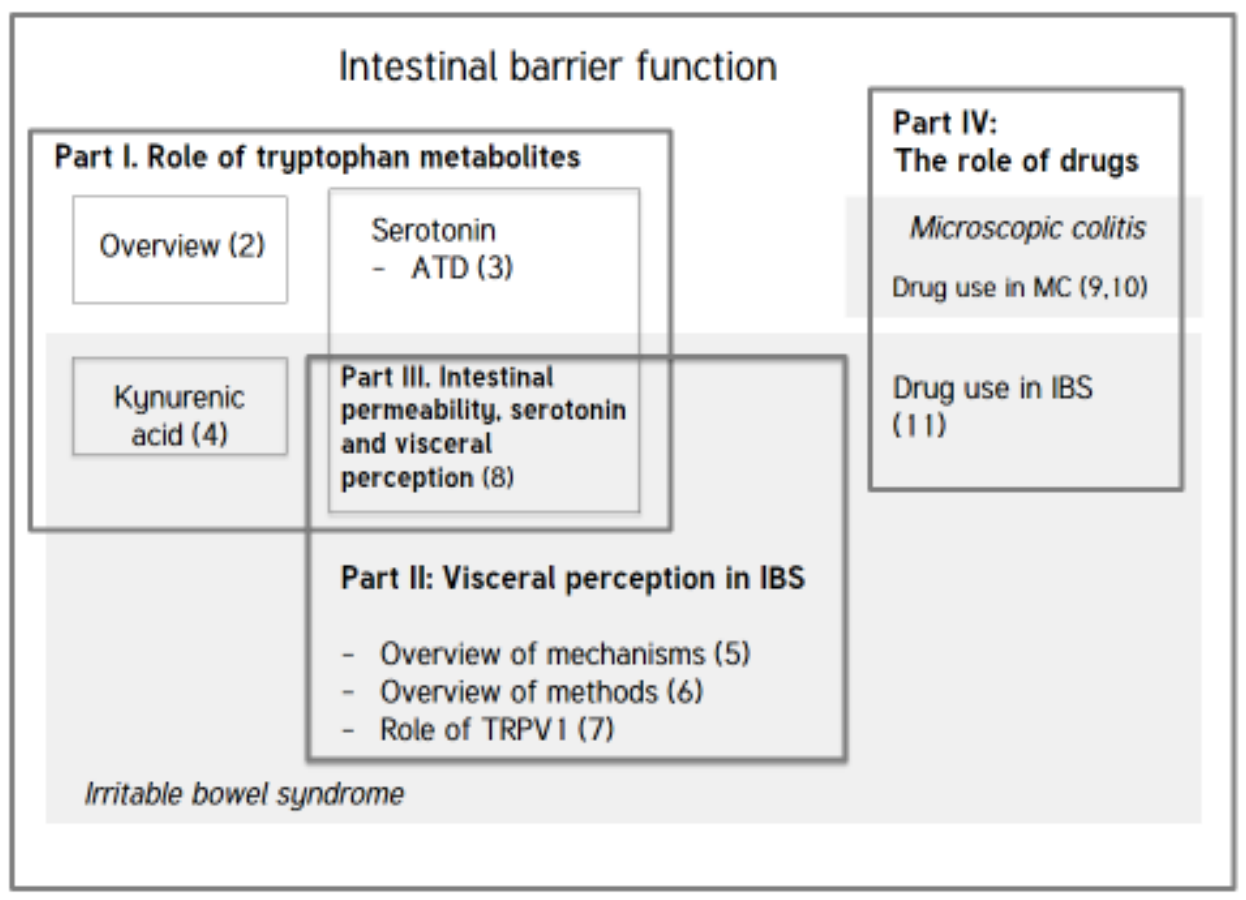

Figure 3: Overview of the thesis.

Part I of this thesis aims to assess the role of tryptophan and its metabolites in gastrointestinal function, and in particular intestinal barrier function (Chapter 2). Tryptophan is an essential amino acid and therefore a principal nutrient, which is required among others for protein synthesis. A small but important proportion of tryptophan is converted to serotonin. Serotonin has a cardinal role in orchestration of intestinal function. It has been demonstrated to initiate secretory and motor reflexes and is also involved in signaling sensory information from the gut to the enteric and central nervous systems. Previous studies have demonstrated profound alterations in serotonergic metabolism in IBS, potentially contributing to the pathogenesis of the disease. Serotonin is also believed to be the key mediator of the interconnection between the intestine and 
the central nervous system, known as the gut-brain axis. Little is known, however, about the potential role of serotonin in modulating intestinal barrier function. We investigated this in both in the in vivo settings (Chapters 3 and 8). In chapter 3, we aimed to negatively influence the serotonergic metabolism by acute tryptophan depletion. In chapter 9, we applied the direct precursor of serotonin, 5-hydroxytryptophan, to positively modulate intestinal serotonin metabolism and assessed effects on intestinal barrier function.

A vast majority of tryptophan is metabolized through the kynurenine pathway. Recent evidence has suggested the involvement of this pathway in IBS. Therefore, we also investigated the putative role of kynurenic acid, a principal kynurenic metabolite, which has been demonstrated earlier to possess anti-inflammatory and neuroprotective properties in the intestine (Chapter 4). Furthermore, the serotonin and kynurenine pathways can compete for their mutual precursor, tryptophan. This implies that diversion of tryptophan to the kynurenine pathway may potentially lead to a relative deficiency of 5-HT synthesis and hence serotonergic dysfunction. Such imbalance has been postulated to play a role in the pathogenesis of IBS. We also investigate this concept of pathway imbalance in this chapter.

Part II of this thesis focuses on the role of visceral perception in IBS, and in particular its relation to intestinal barrier function. Chapter 5 gives an overview of current hypotheses on visceral hypersensitivity in IBS. We also outline some current challenges in terms of investigating visceral perception, with special emphasis in the role in intestinal inflammation, microbiota and serotonin. Chapter 6 summarizes methodological aspects of measuring visceral perception in IBS patients. We critically evaluate the role of rectal balloon distensions in testing visceral hypersensitivity. Also, we give an overview on the most recent developments with regards to brain imaging modalities and neurophysiological recordings and give a comparison of the current methodology.

The perception of pain of visceral origin is a very complex neurobiological process involving a score of nervous elements, which are responsible for converting nociceptive 


\section{Chapter 1}

input from visceral afferents to conscious perception. In this thesis, emphasis is given to the role of peripheral factors of visceral perception, i.e. the role of the visceral afferents and the gastrointestinal mucosa.

In Chapter 7 we investigate the role of certain molecules involved in peripheral pain perception. In this chapter we assess the mucosal transcription of the transient receptor potential vanilloid 1 (TRPV1) receptor. This molecule serves as a pain integrator and is widely expressed throughout the pain neuraxis. Activation of this receptor results in the release of certain neuropeptides from nerve endings, contributing to pro- and antinociceptive processes. We also assess in this chapter the concentration of these peptides in relation to pain symptoms in IBS patients.

Part III of this thesis consists of Chapter 8. This chapter describes an integrative study, in which we simultaneously assess three different pathophysiological aspects in IBS: intestinal permeability, visceral perception and serotonin metabolism - all described to play a certain role in the pathogenesis of IBS. According to one of the current hypotheses on IBS, increased intestinal permeability may contribute to visceral hypersensitivity, as described above. Little is known, however, regarding the potential role of serotonin in relation to this hypothesis. Therefore, in an attempt to gain more insight in the pathophysiology of IBS, we sought to answer the question whether differences in serotonergic signaling underlie the initiation of a cascade of events leading to a reduced epithelial integrity and increased visceral pain perception. In this study, we aimed to specifically modulate the mucosal serotonergic system by administrating the precursor of serotonin, 5-hydroxytryptophan, and measured subsequent effects on intestinal barrier function and visceral perception. We also examined differential responses between IBS patients and healthy controls.

Part IV investigates the potential role for the exposure to certain drugs and the development of diseases related to impairment of the barrier function. Non-steroidal antiinflammatory drugs (NSAIDs) have long been known to possess the ability to impair barrier function. This not related to their inhibitory effect of the cyclooxygenase enzymes, but 
rather a direct toxic effect on the epithelial cells. NSAIDs, already at micromolar concentrations, are able to uncouple oxidative phosphorylation, which reduces intracellular ATP production. This change results in loss of intercellular integrity, as the intercellular junctions are under the control of ATP-dependent actin-myosin complexes, and hence increased intestinal permeability. ${ }^{33}$ Furthermore, experimental analyses have identified a contribution from neutrophils, microcirculatory disturbances, oxygen free radicals, and bile acids in NSAID-induced gastrointestinal damage. ${ }^{12}$

Recent evidence suggests that proton pump inhibitors (PPIs) may also affect intestinal barrier function and thereby contribute to disease pathogenesis. This has been postulated to be related to a) a direct effect on cytoskeletal elements, b) an effect through alteration of intestinal microbiota or c) through electrolyte dysbalance.

Chapter 9 and 10 investigate the potential role of drugs, in particular NSAIDs and PPIs, in the development of microscopic colitis. This condition shares symptomatological characteristics with IBS and increased intestinal permeability has also been demonstrated in these patient. Chapter 9 describes a systematic review of the literature on the putative role of drug exposure in the pathogenesis of microscopic colitis. We also conducted a case-control study (chapter 10), in which we assessed the use of drugs in patients with microscopic colitis in two different centers. Similarly, in Chapter 11, we assessed in a case-control study the putative relation between drug expose and IBS.

Finally, Chapter 12 summarizes the various studies presented in this thesis and discusses the new insights obtained with regards to the role of intestinal permeability, with particular emphasis on its relation to serotonergic metabolism and visceral perception and the pathogenesis of IBS. 
1. Camilleri M, Madsen K, Spiller R, Greenwood-Van Meerveld B, Verne GN. Intestinal barrier function in health and gastrointestinal disease. Neurogastroenterol Motil 2012;24:503-12.

2. Turner JR. Intestinal mucosal barrier function in health and disease. Nat Rev Immunol 2009;9:799-809.

3. Schenk M, Mueller $\mathrm{C}$. The mucosal immune system at the gastrointestinal barrier. Best Pract Res Clin Gastroenterol 2008;22:391-409.

4. Macpherson AJ, Uhr T. Compartmentalization of the mucosal immune responses to commensal intestinal bacteria. Ann N Y Acad Sci 2004;1029:36-43.

5. Shen L, Black ED, Witkowski ED, Lencer WI, Guerriero V, Schneeberger EE, Turner JR. Myosin light chain phosphorylation regulates barrier function by remodeling tight junction structure. J Cell Sci 2006;119:2095-106.

6. Shen L. Tight junctions on the move: molecular mechanisms for epithelial barrier regulation. Ann N Y Acad Sci 2012;1258:9-18.

7. Nusrat A, Turner JR, Madara JL. Molecular physiology and pathophysiology of tight junctions. IV. Regulation of tight junctions by extracellular stimuli: nutrients, cytokines, and immune cells. Am J Physiol Gastrointest Liver Physiol 2000;279:G851-7.

8. Utech M, Ivanov Al, Samarin SN, Bruewer M, Turner JR, Mrsny RJ, Parkos CA, Nusrat A. Mechanism of IFN-gamma-induced endocytosis of tight junction proteins: myosin Il-dependent vacuolarization of the apical plasma membrane. Mol Biol Cell 2005; 16:5040-52.

9. Ye D, Ma I, Ma TY. Molecular mechanism of tumor necrosis factor-alpha modulation of intestinal epithelial tight junction barrier. Am J Physiol Gastrointest Liver Physiol 2006;290:G496-504.

10. Blair SA, Kane SV, Clayburgh DR, Turner JR. Epithelial myosin light chain kinase expression and activity are upregulated in inflammatory bowel disease. Lab Invest 2006;86:191-201.

11. Suenaert P, Bulteel V, Lemmens L, Noman M, Geypens B, Van Assche G, Geboes K, Ceuppens JL, Rutgeerts P. Anti-tumor necrosis factor treatment restores the gut barrier in Crohn's disease. Am J Gastroenterol 2002;97:2000-4.

12. Groschwitz KR, Hogan SP. Intestinal barrier function: molecular regulation and disease pathogenesis. J Allergy Clin Immunol 2009; 124:3-20; quiz 21-2.

13. Drossman DA, Camilleri M, Mayer EA, Whitehead WE. AGA technical review on irritable bowel syndrome. Gastroenterology 2002;123:2108-31.

14. Matricon J, Meleine M, Gelot A, Piche T, Dapoigny M, Muller E, Ardid D. Review article: Associations between immune activation, intestinal permeability and the irritable bowel syndrome. Aliment Pharmacol Ther 2012;36:1009-31.

15. Zhou Q, Verne GN. New insights into visceral hypersensitivity--clinical implications in IBS. Nat Rev Gastroenterol Hepatol 2011;8:349-55.

16. Simren M, Barbara G, Flint HJ, Spiegel BM, Spiller RC, Vanner S, Verdu EF, Whorwell PJ, Zoetendal EG. Intestinal microbiota in functional bowel disorders: a Rome 
foundation report. Gut 2013;62:159-76.

17. Buzza MS, Netzel-Arnett S, Shea-Donohue T, Zhao A, Lin CY, List K, Szabo R, Fasano A, Bugge TH, Antalis TM. Membrane-anchored serine protease matriptase regulates epithelial barrier formation and permeability in the intestine. Proc Natl Acad Sci U S A 2010;107:4200-5.

18. Eswaran S, Goel A, Chey WD. What role does wheat play in the symptoms of irritable bowel syndrome? Gastroenterol Hepatol (N Y) 2013;9:85-91.

19. Wilcz-Villega EM, McClean S, O'Sullivan MA. Mast cell tryptase reduces junctional adhesion molecule-A (JAM-A) expression in intestinal epithelial cells: implications for the mechanisms of barrier dysfunction in irritable bowel syndrome. Am J Gastroenterol 2013;108:1140-51.

20. Gershon MD. 5-Hydroxytryptamine (serotonin) in the gastrointestinal tract. Curr Opin Endocrinol Diabetes Obes 2013;20:14-21.

21. Overman EL, Rivier JE, Moeser AJ. CRF induces intestinal epithelial barrier injury via the release of mast cell proteases and TNF-alpha. PLoS One 2012;7:e39935.

22. Pardi DS, Loftus EV, Jr., Smyrk TC, Kammer PP, Tremaine WJ, Schleck CD, Harmsen WS, Zinsmeister AR, Melton LJ, 3rd, Sandborn WJ. The epidemiology of microscopic colitis: a population based study in Olmsted County, Minnesota. Gut 2007;56:5048.

23. Olesen M, Eriksson S, Bohr J, Jarnerot G, Tysk C. Microscopic colitis: a common diarrhoeal disease. An epidemiological study in Orebro, Sweden, 1993-1998. Gut 2004;53:346-50.

24. Pardi DS, Kelly CP. Microscopic colitis. Gastroenterology 2011;140:1155-65.

25. Abboud R, Pardi DS, Tremaine WJ, Kammer PP, Sandborn WJ, Loftus EV, Jr. Symptomatic overlap between microscopic colitis and irritable bowel syndrome: a prospective study. Inflamm Bowel Dis 2013;19:550-3.

26. Moayyedi P, O'Mahony S, Jackson P, Lynch DA, Dixon MF, Axon AT. Small intestine in lymphocytic and collagenous colitis: mucosal morphology, permeability, and secretory immunity to gliadin. J Clin Pathol 1997;50:527-9.

27. Cremon C, Gargano L, Morselli-Labate AM, Santini D, Cogliandro RF, De Giorgio R, Stanghellini V, Corinaldesi R, Barbara G. Mucosal immune activation in irritable bowel syndrome: gender-dependence and association with digestive symptoms. Am J Gastroenterol 2009; 104:392-400.

28. Fernandez-Banares F, Esteve M, Espinos JC, Rosinach M, Forne M, Salas A, Viver $\mathrm{JM}$. Drug consumption and the risk of microscopic colitis. Am J Gastroenterol 2007;102:324-30.

29. Riddell RH, Tanaka M, Mazzoleni G. Non-steroidal anti-inflammatory drugs as a possible cause of collagenous colitis: a case-control study. Gut 1992;33:683-6.

30. Arrieta MC, Bistritz L, Meddings JB. Alterations in intestinal permeability. Gut 2006:55:1512-20.

31. Bjarnason I, MacPherson A, Hollander D. Intestinal permeability: an overview. Gastroenterology 1995;108:1566-81.

32. Anderson AD, Jain PK, Fleming S, Poon P, Mitchell CJ, MacFie J. Evaluation of a triple sugar test of colonic permeability in humans. Acta Physiol Scand 2004;182:171-7. 
33. Bjarnason I, Takeuchi K. Intestinal permeability in the pathogenesis of NSAIDinduced enteropathy. J Gastroenterol 2009;44 Suppl 19:23-9. 
Introduction 
Understanding the role of tryptophan- and serotonin metabolism in gastrointestinal function

D. Keszthelyi, F.J. Troost, A.A.M. Masclee

Neurogastroenterology and Motility 2009 Dec;21(12):1239-49

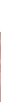




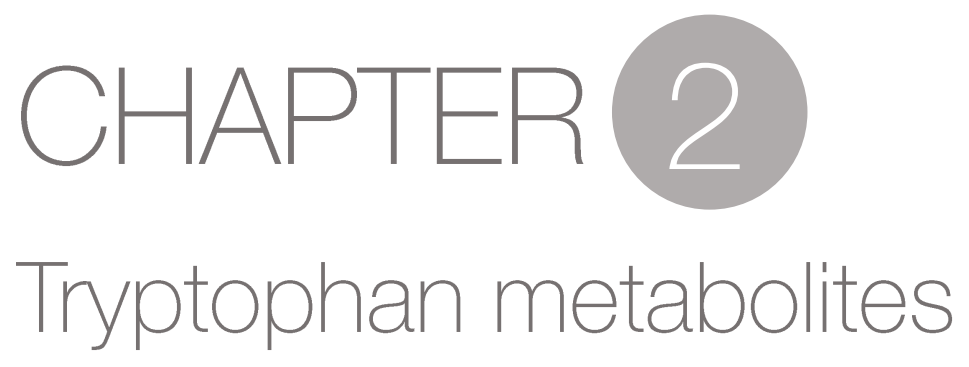

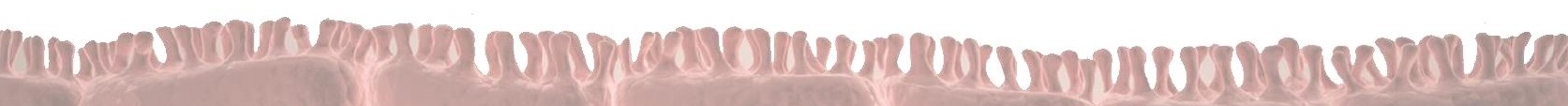




\section{ABSTRACT}

Tryptophan is the precursor of a wide array of metabolites, which are involved in a variety of aspects of human nutrition and metabolism. Accumulating evidence suggests a role of tryptophan metabolites, especially serotonin (5-hydroxytryptamin, 5-HT) in intestinal (patho)physiology, although mechanisms of action are still poorly understood. Alterations of serotonin metabolism may give rise to gastrointestinal dysfunction. Recently, it has been postulated that other metabolites of tryptophan, mostly of the kynurenine pathway, also play a role in regulating gut function. This review analyzes the current knowledge of the interrelationship between tryptophan metabolic pathways and summarizes the existing scientific evidence regarding the role of tryptophan metabolites in intestinal function and in the pathogenesis of gastrointestinal diseases. 


\subsection{INTRODUCTION}

Serotonin (5-hydroxytryptamine, 5-HT), a metabolite of the essential amino acid tryptophan, has been the subject of intense biological research since the early 1950s. With increasing knowledge, it has emerged as a mediator of several functions in the human body, including mood, appetite and hemodynamics. Over the past 20 years, serotonin has also gained recognition in the regulation of gastrointestinal motility, secretion and sensation and has served as a basis for development of novel treatments for gastrointestinal disorders. ${ }^{1,2}$ Recently, the involvement of other tryptophan metabolites in intestinal function has been postulated, which also implicates the clinical relevance of the interrelationship of the different tryptophan metabolic pathways. Of particular importance is the accumulating evidence on the biological role of metabolites of the kynurenine pathway, which are probably involved in the pathogenesis of a number of gastrointestinal disorders. Derangements in this pathway can indirectly lead to changes in serotonin metabolism, which can in turn lead to gastrointestinal dysfunction. We anticipate that the kynurenine pathway will emerge as key modulator in maintaining intestinal homeostasis. Also, metabolic products of human intestinal microbiota will receive increasing attention, By virtue of their catalytic activity, the human gut microbiota have a considerable impact on gastrointestinal function and host health, which is currently subject to intensive investigation. This review will provide a summary of recent developments and will give an insight into the role of tryptophan metabolism and metabolites in intestinal physiology. In addition, clinical implications and perspectives of strategies modulating tryptophan metabolic pathways will be discussed.

\subsection{ABSORPTION OF TRYPTOPHAN}

L-tryptophan is an essential amino acid, with an estimated dietary requirement of 5 $\mathrm{mg} / \mathrm{kg}$ per day. It is the limiting amino acid in nearly all protein sources which are of importance for human nutrition, accounting for 1 to $1.5 \%$ of total amino acids in typical plant and animal proteins, respectively. ${ }^{3}$ Food products containing relatively high tryptophan content are eggs, milk, meat, soybean, potatoes, cereal, broccoli, cauliflower, 
eggplant, kiwi fruit, plums, bananas, walnuts, fish, seafood and tomatoes.

The intestinal absorption of orally ingested tryptophan on the apical membrane of enterocytes is mediated via the $\mathrm{B}^{0} \mathrm{AT} 1$ (Solute Carrier 6A19, SLC6A19) epithelial amino acid transport system, which is also responsible for the absorption of all other neutral amino acids and employs a $\mathrm{Na}^{+}$co-transport mechanism. With the exception of lysine, all other neutral amino acids have a higher affinity to the transport system than tryptophan. A defect in this transport system results in Hartnup disorder, first described in 1956. This is an autosomal recessive disease, characterized by renal aminoaciduria, pellagra-like skin rash and episodes of cerebellar ataxia. An almost complete lack of intestinal tryptophan absorption was demonstrated in vivo and in biopsy material in vitro in patients suffering from this condition, suggesting that the transporter affected in Hartnup disorder is the major mediator of intestinal tryptophan uptake. The pellagra-like skin disorder, which often occurs in Hartnup disorder similar to the classical pellagra caused by dietary niacin deficiency, is the result of the lack of niacin's precursor tryptophan. Impaired absorption leads to the bacterial degradation products indole and derivates, which in turn can play a causative role in cerebellar ataxia. Protein-rich diets usually abolish the symptoms, most likely due to compensation by transport of peptides, although tryptophan breakdown products are still detectable in urine. ${ }^{4}$

The transporter for tryptophan on the basal membrane of enterocytes is the basolateral aromatic amino acid transporter TAT1 (Slc 16a10) protein. This transporter protein is thought to be involved in the pathogenesis of the so-called blue diaper syndrome, whereby malabsorption of tryptophan leads to an overproduction of the bacterial degradation product indican, which is oxidized to indigo causing the blue color of diapers. ${ }^{5}$ To which extent absorption of tryptophan is impaired under this condition remains to be established.

The uptake of tryptophan by peripheral cells, such as tissue macrophages, has not fully been identified yet. Much more is known about tryptophan uptake across the blood brain barrier, which plays a critical role in regulating brain serotonin synthesis. This process is 
based on competitive transport shared by several large neutral amino acids (LNAAs). Therefore, increases in the plasma concentration of LNAAs decrease the rate of tryptophan uptake into the brain and hence brain 5-HT synthesis. ${ }^{6}$ Very little is known about the mechanism underlying uptake into neurons and other cells in the CNS.

\subsection{METABOLISM OF TRYPTOPHAN}

Once in the gastrointestinal tract, tryptophan can enter a number of metabolic pathways: protein synthesis, serotonin pathway, kynurenine pathway and bacterial degradation.

\section{PROTEIN SYNTHESIS}

Given its limited availability in food, tryptophan is often the rate-limiting amino acid in protein synthesis. There is considerable dispute regarding the extent to which dietary tryptophan is incorporated into protein. Some authors suggest a majority of $90 \%,{ }^{3}$ others $30 \%,{ }^{7}$ but other sources agree that there is no net new protein synthesis in steady state nitrogen balanced conditions, therefore, the proportion of dietary tryptophan incorporated in protein is minimal. ${ }^{8}$

\section{SEROTONIN PATHWAY}

About $1-2 \%$ of dietary tryptophan is converted to serotonin. ${ }^{8}$ Serotonin plays an important role in regulating a number of functions in the human body and serves as the precursor for melatonin synthesis in pinealocytes.

\section{KYNURENINE PATHWAY}

The kynurenine pathway is the most tryptophan-consuming metabolic pathway. About $95 \%$ of the ingested tryptophan enters the kynurenine pathway, which can result in the production of NAD, kynuramines, kynurenic acid, quinolinic acid, picolinic acid but most tryptophan is completely metabolized to yield $\mathrm{CO}_{2}$ and ATP via the glutarate pathway. ${ }^{3}$ 


\section{Chapter 2}

bacterial degradation yielding indole, indican, and indole acid derivates. ${ }^{3,} 8$ Approximately $0,5 \%$ of ingested tryptophan is excreted unchanged in urine. ${ }^{8}$

The serotonin and kynurenine pathways as well as the bacterial degradation of tryptophan will be discussed in detail below. Figure provides a schematic representation of these different metabolic pathways.

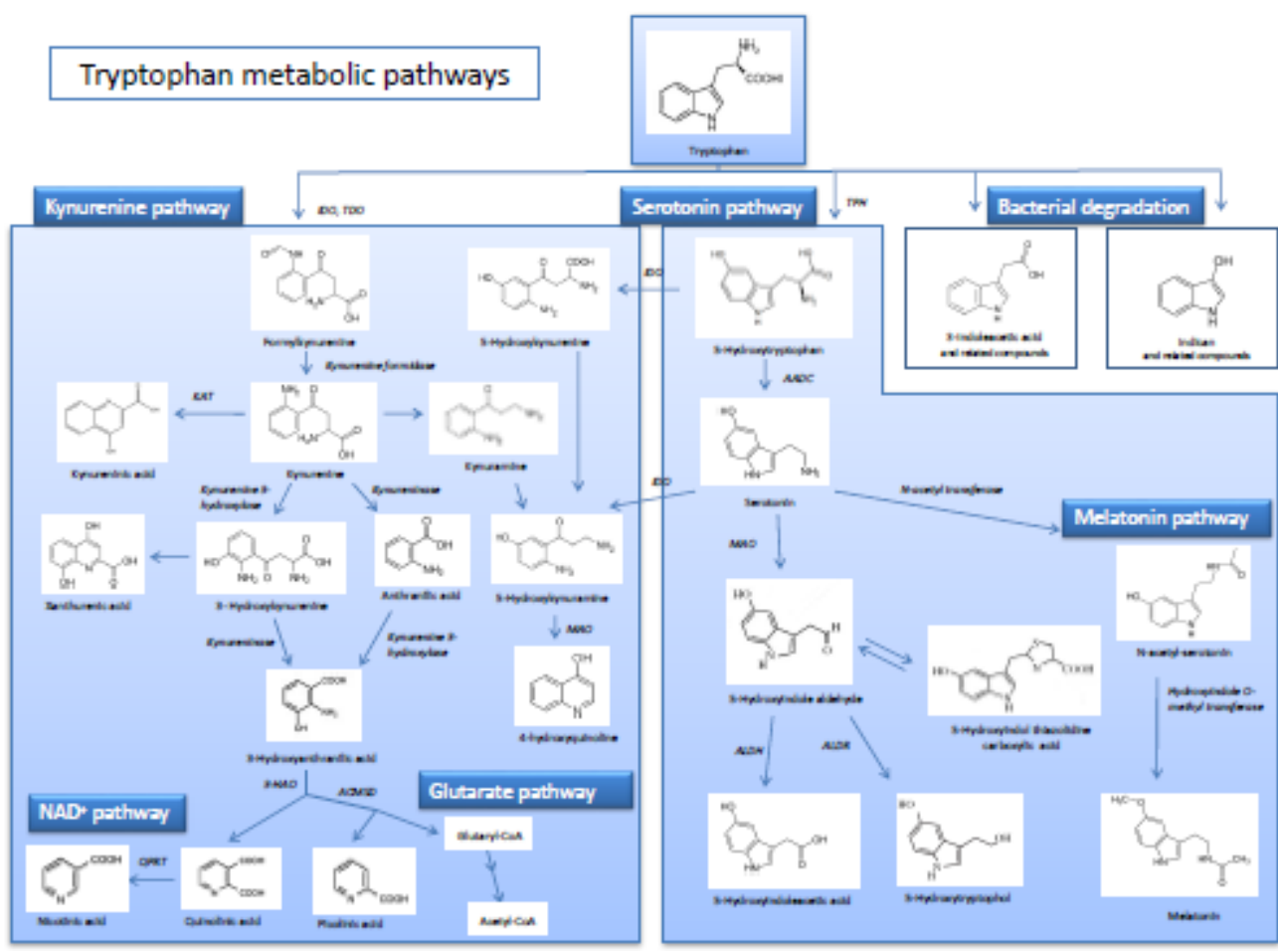

Figure 1: Tryptophan metabolic pathways. TDO, tryptophan 2,3-dioxygenase; IDO, indoleamine 2,3-dioxygenase; KAT, kynurenine aminotransferase;

\subsection{SEROTONIN PATHWAY}

Synthesis - There is quite extensive knowledge on the importance of serotonin in regulating gastrointestinal function. ${ }^{1,2}$ It is one of the most important signaling molecules within the gut, where it plays a pivotal role in initiating secretory and motor reflexes. 
Approximately $95 \%$ of the total serotonin content of the human body is present in the gut, of which $90 \%$ is stored in the enterochromaffin cells of the intestinal mucosa. The remaining $10 \%$ is largely attributed to serotonergic neurons of the ENS. These cells possess the apparatus to produce and store serotonin and hence play an important role in regulating serotonin homeostasis., 2 Platelets are unable to synthesize serotonin themselves, but possess a high-affinity uptake system and hence accumulate high concentrations of serotonin derived from the Gl tract.

Serotonin is synthesized from tryptophan through hydroxylation and decarboxylation. These processes are catalyzed by the tryptophan hydroxylase (TPH) and the aromatic acid decarboxylase (AADC), respectively, the former being the rate-limiting step in the synthesis.1, 2 Tryptophan hydroxylase (tryptophan 5-monooxygenase) expression is limited to a few specific cells: neurons, pinealocytes, mast cells, mononuclear leukocytes, intestinal enterochromaffin cells and bronchopulmonary neuroendocrine epithelial cells. There are two known isoforms of TPH. TPH1 is localized in enterochromaffin cells and the pineal gland and $\mathrm{TPH} 2$ is present in neurons, including serotonergic neurons of the ENS. ${ }^{9}$ Both isoforms employ molecular oxygen and the cofactor tetrahydrobiopterin to convert tryptophan to 5-hydroxytryptophan (5-HTP), which is then converted by AADC to serotonin. AADC, which employs vitamin B6 as cofactor, is found in all aminergic cells of the central and peripheral nervous system and is distributed widely in non-neural tissue. ${ }^{10}$

There are at least 14 different neuroendocrine cells of the Gl tract, one of them being the serotonin-producing enterochromaffin cells. Serotonin is stored in large dense core vesicles and synaptic-like microvesicles in enterochromaffin cells and is released as result of mechanical or chemical stimulation. ${ }^{11}$ Enterochromaffin cells function as sensory transducers. Serotonin released from enterochromaffin cells stimulates mucosal nerve endings of intrinsic primary afferent neurons of the enteric nervous system, which coordinate secretory and motor functions of the intestine. ${ }^{1,2}$ Serotonin also activates vagal afferent nerve endings of extrinsic primary afferent neurons projecting to the 
vomiting center in the brainstem.'

Carcinoids are rare tumors derived from enterochromaffin cells. The carcinoids are most commonly observed in the GI tract and the bronchopulmonary system, which can produce bioactive amines such as serotonin. A subset of patients with carcinoid tumors suffer from carcinoid syndrome, characterized by flushing, diarrhea, cramps, skin abnormalities, asthmatic wheezing and valvular heart disease associated with elevated $5-\mathrm{HT}$ levels. ${ }^{12}$ In carcinoid syndrome patients, an overwhelming majority of tryptophan is converted to serotonin. Some foregut carcinoid tumors are AADC-deficient, and are therefore unable to convert 5-HTP to 5-HT, and may consequently secrete 5-HTP and to lesser extent serotonin. ${ }^{13}$ Increased rates of serotonin turnover indicated by elevated urinary 5hydroxy indoleacetic acid (5-HIAA), a major metabolite of serotonin, can lead to depletion of tryptophan as precursor for other metabolic pathways leading to conditions such as pellagra-like symptoms due to deficient niacin synthesis. ${ }^{8}$ A weak inhibitor of TPH, parachlorophenylalanine (pCPA) has proven effective in the treatment pellagra and diarrhea in carcinoid syndrome patients as well as in chemotherapy induced emesis. ${ }^{14}$ Unfortunately, administration of pCPA has been associated with the onset of depression and other alterations in CNS function. Recently, a novel class of gastrointestine-selective TPH inhibitors has been discovered, which may provide potential treatment for a variety of gastrointestinal diseases caused by the dysregulation of the serotonergic pathway. ${ }^{15}$

Serotonergic neurons are the minor source of serotonin in the gastrointestinal tract. Small populations of interneurons that can synthesize, take up and release $5-\mathrm{HT}$ have been identified in the human ENS. ${ }^{1}$ In guinea pigs, these neurons constitute $2 \%$ of the total amount of neurons in the myenteric plexus and their targets project to other submucosal and myenteric ganglia. About half of the myenteric neurons receive serotonergic input and the serotonergic interneurons themselves are targets for 5-HT innervations. ${ }^{16}$ Serotonergic interneurons play a role in both motor and secretory reflexes by providing synaptic inputs to motor neurons and ascending interneurons. ${ }^{17}$ 


\section{METABOLISM OF SEROTONIN}

Serotonin is metabolized by several metabolic pathways. The majority of serotonin is catabolized by monoamine oxidase (MAO). This enzyme is located in mitochondria of a wide range of cells and catalyzes oxidative deamination of several biogenic amines. At least two forms of MAO are known (MAO-A and MAO-B). MAO-A has the highest affinity for serotonin. ${ }^{18}$

5-hydroxyindole acetaldehyde (5-HIAL), the product of oxidative deamination of serotonin can be metabolized to 5-hydroxy indoleacetic acid (5-HIAA) or 5hydroxytryptophol (5-HTOL). The former reaction is the major pathway under normal conditions. This reaction is catabolyzed by aldehyde dehydrogenase, employing NAD as coenzyme. Reduction of the aldehyde intermediate is catalyzed by aldehyde reductase, which employs NADH as coenzyme. ${ }^{19}$ In case of a 5-HT overload, the known 5-HT catabolic pathways may become overloaded, allowing lesser-used pathways to convert more $5-\mathrm{HT}$ thereby increasing levels of $5-\mathrm{HT}$ metabolites that might not readily be observed under other conditions.

5-hydroxytryptophol (5-HTOL) is a minor serotonin metabolite under normal conditions, accounting for $1 \%$ of the total serotonin turnover. Alcohol consumption leads to an increased synthesis of 5-HTOL and a concomitant decrease in the synthesis of 5-HIAA, resulting in an increased 5-HTOL/5-HIAA ratio, which is a sensitive marker for detection of recent alcohol intake. During ethanol oxidation the conversion of serotonin shifts away from oxidation of the intermediate 5-HIAL producing 5-HIAA toward the reductive pathway forming 5-HTOL. This has been attributed to the competitive inhibition of aldehyde dehydrogenase by ethanol-derived acetaldehyde. ${ }^{19}$

Another proposed metabolic pathway of serotonin involves glucuronidation. Serotonin has been characterized as a highly selective substrate of human UDPglucuronosyltransferase UGT1A6 in Caco-2 cells, an in vitro model of human intestinal epithelium. These findings suggest that UGT1A6 contributes to the homeostatic control 
of intestinal 5-HT metabolism. ${ }^{20}$ However, studies on the expression of UGT IA6 in human intestinal epithelium are not conclusive. Munzel et al. demonstrated the expression of UGT1A6 mRNA in human duodenum with large interindividual differences, ${ }^{21}$ while Radominska-Pandya et al..$^{22}$ found that UGT1A6 was not present in the human intestine, suggesting a regulational control of intestinal $5-\mathrm{HT}$ metabolism that remains to be elucidated.

Recently, the metabolite 5-hydroxyindole thiazolidine carboxylic acid (5-HITCA) has been identified in rodent CNS and ENS tissue samples. This is a condensation product of 5hydroxyindole acetaldehyde and L-cysteine. ${ }^{23} 5$-HITCA can be detected natively in homogenizated rodent ENS samples. In 5-HT incubated central and enteric nervous system tissue samples, 5-HITCA forms at levels equivalent to 5-HIAA. An equilibrium between 5-HITCA and 5-hydroxyindole acetaldehyde coupled to the enzyme aldehyde dehydrogenase, in the CNS and ENS suggests equilibrium prevents this accumulation.23 Imbalances in 5-HT levels within the ENS and the intestine have been associated with various functional gastrointestinal disorders. The most attention perhaps has been given to irritable bowel syndrome, which has been documented extensively.1, 24 Irritable bowel syndrome (IBS), affecting approximately $15-20 \%$ of the adult population, is a functional intestinal disorder characterized by abdominal pain or discomfort associated with altered bowel habits, without indications for an organic cause. An increased number of enterochromaffin cells as well as altered mucosal serotonin metabolism have been described in IBS and serotonergic compounds have been shown to beneficially influence intestinal motor and sensory function.' A functional hallmark of IBS is visceral hypersensitivity, which is present in approximately $50 \%$ of patients with IBS ${ }^{25}$. Previous research highlights the involvement of the $5-\mathrm{HT}_{3}$ receptors on extrinsic primary afferent neurons of the ENS in mediating the pain sensation. ${ }^{1}$ It was also suggested previously that the increased visceral sensitivity observed in IBS patients may at least partly be caused by a decreased epithelial integrity, causing intraluminal compounds to cross the epithelial barrier and trigger the ENS and, thereby, nociception.26 Serotonin could therefore also play a role in regulating the permeability of the intestine. 
Tight regulation of 5-HT levels in nervous tissue and intestinal mucosa is necessary and 5-HT catabolism plays an important role in this regulation. Understanding the catabolic pathways, and knowledge on the enzymes involved and the products of these conversions are particularly important because catabolism is vital to regulation of 5-HT levels. Elucidating 5-HT synthetic and catabolic pathways may provide novel approaches for the therapies designed to treat disorders associated with 5-HT homeostasis.

\subsection{KYNURENINE PATHWAY}

In adult young men, about $95 \%$ of dietary tryptophan is metabolized along the kynurenine pathway. ${ }^{3}$ The biological functions of the kynurenine pathway are: clearance of excess tryptophan and regulation of plasma tryptophan levels, maintenance of nicotinic acid levels, regulation of CNS function and enhancement of macrophage defense function.

Entering the kynurenine pathway, tryptophan is first oxidized by tryptophan 2,3dioxygenase (TDO), which is almost entirely localized at hepatic cells. TDO is the rate limiting enzyme for kynurenine synthesis in the periphery. TDO expression and activity can be induced four- to tenfold by tryptophan loading within a period of a few hours. ${ }^{8}$ The principal branch of the kynurenine pathway generates quinolinic acid and nicotinamide, whereas the side chains generate kynurenic acid and xanthurenic acid (see Figure 1). Several biological features of kynurenine metabolites have been described. Most attention has been given to the imbalance in neurotoxic and neuroprotective properties of these compounds, which have been associated with several CNS pathologies. Quinolinic acid is considered to be an excitotoxic N-methyl D-aspartate (NMDA) receptor agonist, whereas kynurenic acid is a neuroprotective NMDA antagonist and an $\alpha 7$ nicotinic cholinergic agonist. ${ }^{27}$ In mononuclear cells, including tissue macrophages, quinolinic acid is the main end product of the kynurenine pathway and plays a role in immunoregulatory processes. ${ }^{28}$

The kynurenine pathway also provides the precursors for the dietary supplement niacin, a collective term for nicotinamide and nicotinic acid. Under normal conditions, most of the 
tryptophan that enters the oxidative pathway is converted to $\mathrm{CO}_{2}$ and water in the glutarate pathway. Only if this branch of the pathway is saturated, NAD becomes a major product of metabolism. ${ }^{3}$ Although metabolites of the glutarate pathway are present in many tissues, including the intestine, NAD synthesis is only possible in the liver, because this is the only organ that possesses all the necessary enzymes. ${ }^{28}$

Another product of the kynurenine pathway is picolinic acid. Picolinic acid is only produced when the flux of metabolites through the glutarate pathway is high and enzymes of the glutarate pathway are saturated. ${ }^{3}$ Picolinic acid acts as a chelating agent of elements such as chromium, zinc, manganese, copper, iron, and molybdenum in the human body. It forms a complex with zinc that may facilitate the passage of zinc through the gastrointestinal wall and into the circulatory system. ${ }^{8}$ Several of the enzymes of the kynurenine pathway use vitamin B6 as cofactor and a key feature of the enzyme kynureninase is its exceptionally high sensitivity to pyridoxine deficiency. Lack of vitamin $\mathrm{B} 6$ leads to a large increase in xanthurenic acid excretion. This has been used for decades as a diagnostic test for vitamin B6 deficiency. ${ }^{8}$ Vitamin B6 deficiency also compromises serotonin synthesis, and hence can lead to competition between the two pathways for the co-factor.

Besides TDO, another enzyme initializing the kynurenine pathway is indoleamine 2,3dioxygenase (IDO). IDO is widely distributed in peripheral tissues. The human intestine contains a relatively large amount of IDO.29 While TDO exclusively accepts tryptophan as substrate, IDO has a broader specificity and can also take 5-HTP, 5-HT and tryptamin.9 The expression of IDO increases in response to infection and inflammation, with interferon-y being the strongest stimulator. Mononuclear cells that synthesize IDO reduce extracellular tryptophan concentration so that adjacent T-cells, which depend on tryptophan from the extracellular environment, are unable to activate and proliferate upon encountering antigens. Therefore, IDO might play a role in preventing the initiation of autoimmune disease by enforcing T-cell tolerance through suppressing their proliferation. ${ }^{28}$ Hence, high local expression of IDO by mononuclear cells may represent 
an anti-inflammatory and immunosuppressive mechanism tempting to counterbalance tissue damage. ${ }^{30}$ This mechanism could be involved in intestinal pathophysiology, since IDO expression is markedly induced in lesional colonic biopsies of inflammatory bowel disease (IBD) patients ${ }^{30}$ and increased IDO activity has been observed in patients with celiac disease ${ }^{31}$ and diverticulitis. ${ }^{32}$ A similar IDO-based intrinsic immunoescape mechanism is probably employed by colon tumor cells. ${ }^{33}$

Besides through the regulatory effect of IDO on T-cells and immune function, inflammatory responses in the ENS and the gastrointestinal tract related to the kynurenine pathway can also be based on a sensitive balance between the proinflammatory, excitotoxic quinolinic acid and the anti-inflammatory, neuroprotective kynurenic acid. ${ }^{34}$ This balance could have profound influence on the excitability of enteric neurons, which can affect intestinal motor and sensory function. Increased levels of the kynurenine pathway metabolites kynurenine and kynurenic acid have been observed in sera of patients with inflammatory bowel disease (IBD). 35 The increased activity of the kynurenine pathway may represent either a compensatory response to elevated activation of enteric neurons or a primary abnormality which induces a compensatory increase in gut activity. In either case, the data may indicate a role for the kynurenine modulation of glutamate receptors in the symptoms of IBD. ${ }^{35}$

Recent evidence suggests the involvement of the kynurenine pathway in intestinal motility, although the exact roles of kynurenine metabolites in intestinal motor function remains unclear. ${ }^{34}$ Kynurenic acid acts as an antagonist on NMDA receptors on enteric glutamatergic neurons and may cause dysregulation of intestinal motility. Glutamate is likely to play an excitatory role and may modulate cholinergic transmission in the ENS.36 Glutamate immunoreactivity has been detected in submucosal and myenteric neurons in the guinea pig ileum, and NMDA receptors are present on enteric cholinergic neurons, as well as vagal and spinal primary afferent nerve endings. NMDA receptor activation has been shown to stimulate acetylcholine release from myenteric neurons, thereby modulating smooth muscle contraction. ${ }^{36}$ Recent results have revealed a significant 
potential for kynurenic acid to decrease the facilitary pathways of colonic motility. ${ }^{34}$ Kynurenic acid might also play a role in intestinal mechanosensitivity, as it has been proven to act also as glutamate antagonist and inhibit mechanosensitivity of both mucosal and tension vagal afferents. ${ }^{37}$ Furthermore, kynurenic acid also exerts an antiinflammatory effect due to inhibition of xanthine oxydase, resulting in less reactive oxygen species (ROS) production. ${ }^{34}$ Other products of the IDO and formamidases are kynuramin derivates. Formation of kynuramines has been described in various tissues, including the intestine and appears to be directly proportional to tryptophan concentrations. Kynuramines may be important as endogenous agonists or antagonists of 5 -HT receptors in smooth muscle. Marked non-selective serotonergic agonist properties of 5-hydroxykynuramine at multiple 5-HT receptors were demonstrated in rat ileum. 5-hydroxykynuramine is formed from tryptophan to much lesser extent in vivo than $5-\mathrm{HT}$, but pathological conditions or situations in which tryptophan concentrations are increased may lead to an overproduction of kynuramines. Besides its effect on smooth muscle, 5-hydroxykynureamine is a potent inhibitor of the action of serotonin in promoting the aggregation of platelets. This may provide a measure of regulation in cases of over-synthesis of serotonin, not only as an alternative catabolic pathway of the amine, but also to inhibit one of its biological actions. ${ }^{38}$

\subsection{BACTERIAL DEGRADATION PRODUCTS}

Indican - The main bacterial breakdown product of tryptophan is indole. The wide range of bacterial species capable of producing indole include E. coli, Proteus vulgaris, Paracolobactrum coliforme, Achromobacter liquefaciens and Bacteriodes spp. The formation of indole is catabolyzed by the enzyme tryptophanase, which is inducible by tryptophan and repressible by glucose in most bacteria. ${ }^{39}$ By-products of this conversion are pyruvate, which can be used in fermentation or respiration reactions, and ammonia, which can have potentially toxic effects on the intestinal epithelium. High protein diets are therefore able to induce bacterial tryptophanase activity, which in turn results in overproduction of indole and other compounds that can thereby reach toxic 
concentrations in the colon. ${ }^{39}$

After absorption, indole is oxidized to indoxyl, conjugated with sulphate and excreted as urinary indican (also known as indoxyl-sulphate). In normal individuals only small proportions of dietary tryptophan reach the colon because of nearly complete absorption in the small intestine. Approximately $3 \%$ of dietary tryptophan is excreted as urinary indicant. ${ }^{8}$ Because the upper gastrointestinal tract is sparsely populated with bacteria, indican is present in urine at low levels in healthy individuals. An elevated level of urinary indican can be an indication of upper bowel bacterial overgrowth. Nevertheless, even patients with normal intestinal bacterial populations can show increased postprandial indican excretion when they fail to digest dietary protein. Although not used in everyday medical practice due to availability of more specific tests, the relationship between increased indican and incomplete digestion may serve as measure of protein digestive adequacy (Obermeyer test). ${ }^{8}$

Indican is also known to be a nephrotoxin that accumulates in the blood of patients suffering from chronic kidney failure. Because tryptophanase activity derives from only a subset of enteric bacteria, non-indole-producing bacteria, such as various Bifidobacterium species, have been administered as a test probiotic to dialysis patients to decrease their plasma levels of indoxyl sulfate. ${ }^{40}$ Excessive indican excretion also occurs in a rare condition known as the purple urine bag syndrome, first described in 1987. It is associated with urinary tract infections occurring in catheterized patients, generally elderly females with significant co-morbidities and constipation, in which case excess indican is transformed to indirubin and indigo in alkaline urine in the presence of sulphatase/phosphatase producing Gram negative bacteria. ${ }^{41}$

Indolic acid derivates - A smaller quantity of tryptophan is converted by bacterial action to indolic acid derivates: indolyl-3-acetic acid, indolyl-acetyl-glutamine, indolyl-propionic acid, indolyl-lactic acid, indolyl-acrylic acid and indolyl-acryloyl-glycine. Intestinal microorganisms, including Bacteriodes, Clostridia and E.coli, catalyze tryptophan to tryptamin and indolyl-pyruvic acid, which are then converted to indolyl-3-acetic acid, 
indolyl propionic acid and indole lactic acid..$^{39}$ Indolyl acetic acid can be conjugated with glutamine in the liver to yield indolylacetyl glutamine. Indolylpropionic acid can be further converted in the liver or kidney into indolyl acrylic acid (IAcrA) and conjugated with glycine to produce indolylacryloyl glycine (IAcrGly). Some evidence suggests that IAcrA can also be produced in the absence of intestinal microorganisms, although there is no direct evidence for enzymatic or non-enzymatic processes..$^{42}$ Nutritional intervention such as tryptophan loading did not influence urinary IAcrGly levels, but complete elimination of tryptophan from the diet resulted in a marked decrease of IAcrGly in urine, similarly to parenteral alimentation. ${ }^{42}$

The biological role of these compounds still needs to be investigated. Increased and prolonged excretion of urinary indols (e.g. indican, indolyl-3-acetic acid, indolyl-3-acetylglutamine, indolyl-lactic acid, indolyl-acryloyl-glycine) has been observed in a number of diseased states including Hartnup disorder, celiac disease and other malabsorptive states. ${ }^{43}$ It is assumed that this is due to excessive tryptophan overload in the colon possibly with coexistent alteration in gut microbiota, which leads to increased production of bacterial degradation products. ${ }^{43}$

Some bacterial products are toxic to other microbiota, and this provides competitive advantage for the producers. Some indolic compounds are known to have bacteriostatic effect on Gram negative enterobacteria, especially within the genera Salmonella and Shigella. ${ }^{39}$ The increased urinary excretion of indolic compounds reflects variations in gut microbiota composition in relation to nutritional competition. For instance, indolyl acetic acid has been reported to inhibit the growth and survival of Lactobacilli, and specifically L. paracasei. ${ }^{44}$ Also, indolyl propionic acid has been shown to be a powerful antioxidant, and is currently being investigated as a possible treatment for Alzheimer's disease. ${ }^{40}$

Some authors suggest that indolylacryloyl glycine could be a possible marker for autism and is also associated with increased intestinal epithelial permeability. ${ }^{45}$ Autism has been associated with increased intestinal permeability. ${ }^{46}$ Researchers from Sunderland, UK, hypothesized that the changes in intestinal permeability are a result of membrane 
damage through the precursor of IAcrGly, indolyl acrylic acid, a planar and very reactive molecule. Elevated levels of IAcrA are detectable in urine by measuring its metabolite IAcrGly. The membrane dysfunction results in an increased permeability and permeation of compounds which could influence normal homeostasis, including CNS development, which may result in pervasive developmental disorders or autism. ${ }^{45}$ This group has also suggested a gluten-free diet which decreases the urinary levels of IAcrGly and ameliorates some of the symptoms of the pervasive developmental disorder. Their theory is very plausible, but not generally accepted. ${ }^{47}$

\subsection{COMMON GASTROINTESTINAL DISORDERS}

Recent studies suggest the involvement of tryptophan metabolic routes in the pathogenesis of several common gastrointestinal disorders, such as IBD, IBS, celiac disease and diverticulitis.

A common mechanism could possibly be the upregulation of IDO. Elevated kynurenine and kynurenine/tryptophan ratios, indicative of increased IDO activity, have been observed under inflammatory conditions, such as IBD, which result in altered T-cell proliferation and survival. ${ }^{30}$ Such a mechanism could also account for altered tryptophan catabolism in IBS patients, as studies have reported immune activation and proinflammatory cytokine production in IBS. ${ }^{48}$

Moreover, upregulation of IDO induces a metabolic shift, which is presumably also involved in the pathogenesis of IBS. Recent studies have shown that both females ${ }^{49}$ and males $^{50}$ with IBS have increased kynurenine concentrations compared to controls. Furthermore, a positive correlation was found between IBS severity and the kynurenine/tryptophan ratio. Those with severe IBS symptoms have increased shunting of tryptophan along the kynurenine pathway which contributes to the abnormal serotonergic function. ${ }^{49}$ Altered serotonergic conditions have directly been associated with malfunction of the intestine in IBS. 


\section{Chapter 2}

Besides a role in the pathological states of the intestines, serotonin has also been suggested to be involved in the pathogenesis of non-alcoholic steatohepatitis (NASH). Degradation of serotonin by MAO also yields ROS as byproducts. Increased uptake and catabolism of serotonin in the liver therefore leads to overproduction of ROS resulting in hepatocellular injury by mitochondrial damage and inflammation. ${ }^{2}$ In recent years, the understanding of the (patho)physiological role of tryptophan metabolites, mostly serotonin, in the gastrointestinal tract has increased significantly.1, 2, 31, 34, 35, 49 Nevertheless, further investigation will be needed to assess the biological role of other tryptophan derivates. This will lead to a better comprehension of the pathogenesis of the most common causes of gastrointestinal morbidity.

\subsection{NUTRITIONAL MODULATION OF TRYPTOPHAN METABOLISM}

The question arises to which extent tryptophan and serotonin metabolism can be influenced by nutrition and how this affects gut function. In-depth understanding of pathways involved in tryptophan and serotonin metabolism is therefore crucial. Furthermore, the interrelationship between metabolic pathways is of profound pharmacological and physiological importance, in that changes in one pathway might have secondary effect on the others.

Nutritional intervention has been employed since decades in investigating the serotonergic system, which has been proven to be involved in the pathogenesis of several psychiatric disorders, mainly depression. It is also well known that depression and other psychiatric disorders often coincide with functional gastrointestinal disorders and that serotonin is a mediator between brain and intestine, the so-called brain-gut axis. ${ }^{51}$ Furthermore, serotonin also has a pivotal role in regulating appetite, satiety and food intake. ${ }^{52}$

Experimental evidence in humans suggests that an acute decrease in 5-HT synthesis can be achieved by means of the acute tryptophan depletion (ATD) method. ATD employs ingestion of an amino acid mixture devoid of the precursor of $5-\mathrm{HT}$ and subsequently 
lowers 5-HT synthesis between 4 and 7 hours after administration, based on a competitive uptake between tryptophan and other large neutral amino acids. ATD has been used over the past decade in the psychiatric setting; recent evidence suggests that it affects the brain-gut axis and intestinal 5-HT metabolism as well. Using the ATD method, alteration of gastrointestinal and anxiety symptoms, ${ }^{53}$ as well as changes in visceral perception and cognition have been observed in IBS patients. ${ }^{54}$

Historically, tryptophan has been used as an anti-depressive and sleep-inducing substance through increasing $5-\mathrm{HT}$ synthesis. ${ }^{55}$ Similar effects have been observed in case of supplementation with a tryptophan-rich protein, alpha-lactalbumine ${ }^{56}$ and nutritionally-sourced tryptophan. ${ }^{57}$ Furthermore, tryptophan loading increases the catabolism along the kynurenine pathway and can lead to substrate depletion from the serotonin pathway. A similar metabolic shift and subsequent shortage of 5 -HT has also been postulated to be responsible for major depression. ${ }^{58}$ Inflammatory conditions also lead to induction of metabolism along the kynurenine pathway. Therefore, the direct precursor of serotonin, 5-hydroxytryptophan (5-HTP), has also been used to influence the serotonergic system. A natural source of 5-HTP is the seeds of the West-African plant Griffonia simplicifolia. Following oral intake, it is converted by the intestine and liver to serotonin by the action of AADC. ${ }^{10}$ Therefore, it can be expected that administration of 5-HTP causes an increase in the intestinal 5-HT levels. A small amount of 5-HTP is taken up by the central nervous system, the reason for its use as an anti-depressant. ${ }^{55}$

Evidence also suggests that the kynurenine pathway itself can be influenced by nutrition. ${ }^{28}$ Kynurenic acid has been proposed to have a positive effect in conditions accompanied with intestinal hypermotility. ${ }^{34}$ It is present in certain sources of food, such as honeybee products. ${ }^{59}$ Therefore, kynurenic acid and its derivates may be used as a therapeutic target for the treatment of functional gastrointestinal disorders, ${ }^{34}$ possibly through nutritional intervention.

Imbalance between neuroprotective and neurotoxic activities of the kynurenine pathway have been associated with various CNS pathologies. ${ }^{27}$ Based on recent scientific evidence 


\section{Chapter 2}

as discussed above, an imbalance of kynurenine pathway metabolites also need to be considered in the development of gastrointestinal dysfunction and intestinal immune function. The biological effects of bacterial degradation products of tryptophan need to be further clarified as well. An overload of dietary proteins or changes in intestinal microbiota can lead to overproduction of these products and subsequently lead to changes in intestinal physiology with potentially toxic consequences. Interest in the impact of gut microbial activity on human health is expanding rapidly, and a better identification of the host-microbiota interactions can also lead to developing new approaches in disease treatment. Probiotic supplementation, for instance, can aim to replace or reduce the number of potentially harmful proteolytic E.coli and Clostridia producing toxic tryptophan breakdown products by enriching populations of gut microbiota that have more advantageous metabolic activity. Future activities will be directed to influence the gut microbiota in a targeted way, ideally by enhancing beneficial effects and minimizing adverse effects. However, before we are able to do so, further work is required to understand in more detail the processes underlying the bacterial conversion of tryptophan and other dietary components. Given our current knowledge of tryptophan metabolism in the human body, we may assume that nutritional modulation can offer a tool to better understand gastrointestinal functioning and also to open new therapeutic horizons in the treatment of functional gastrointestinal disorders. 


\section{9 .}

REFERENCES

1. Gershon MD, Tack J. The serotonin signaling system: from basic understanding to drug development for functional GI disorders. Gastroenterology 2007;132:397-414.

2. Lesurtel M, Soll C, Graf R, Clavien PA. Role of serotonin in the hepatogastrolntestinal tract: an old molecule for new perspectives. Cell Mol Life Sci 2008;65:940-52.

3. Peters JC. Tryptophan nutrition and metabolism: an overview. Adv Exp Med Biol 1991;294:345-58.

4. Broer S. Amino acid transport across mammalian intestinal and renal epithelia. Physiol Rev 2008;88:249-86.

5. Drummond KN, Michael AF, Ulstrom RA, Good RA. The Blue Diaper Syndrome: Familial Hypercalcemia with Nephrocalcinosis and Indicanuria; a New Familial Disease, with Definition of the Metabolic Abnormality. Am J Med 1964;37:928-48.

6. Fernstrom JD. Branched-chain amino acids and brain function. J Nutr 2005; 135:1539S-46S.

7. Allegri G, Costa CV, Bertazzo A, Biasiolo M, Ragazzi E. Enzyme activities of tryptophan metabolism along the kynurenine pathway in various species of animals. Farmaco 2003;58:829-36.

8. Bender DA. Biochemistry of tryptophan in health and disease. Mol Aspects Med 1983;6:101-97.

9. Walther DJ, Peter JU, Bashammakh S, Hortnagl H, Voits M, Fink H, Bader M. Synthesis of serotonin by a second tryptophan hydroxylase isoform. Science 2003;299:76.

10. Chang YT, Mues G, Hyland K. Alternative splicing in the coding region of human aromatic L-amino acid decarboxylase mRNA. Neurosci Lett 1996;202:157-60.

11. Rindi G, Leiter AB, Kopin AS, Bordi C, Solcia E. The "normal" endocrine cell of the gut: changing concepts and new evidences. Ann N Y Acad Sci 2004;1014:1-12.

12. Modlin IM, Lye KD, Kidd M. A 5-decade analysis of 13,715 carcinoid tumors. Cancer 2003;97:934-59.

13. Oates JA, Sjoerdsma A. A unique syndrome associated with secretion of 5hydroxytryptophan by metastatic gastric carcinoids. Am J Med 1962;32:333-42.

14. Alfieri $A B$, Cubeddu LX. Treatment with para-chlorophenylalanine antagonises the emetic response and the serotonin-releasing actions of cisplatin in cancer patients. Br J Cancer 1995;71:629-32.

15. Shi ZC, Devasagayaraj A, Gu K, Jin H, Marinelli B, Samala L, Scott S, Stouch T, Tunoori A, Wang Y, Zang Y, Zhang C, Kimball SD, Main AJ, Sun W, Yang Q, Nouraldeen A, Yu XQ, Buxton E, Patel S, Nguyen N, Swaffield J, Powell DR, Wilson A, Liu Q. Modulation of peripheral serotonin levels by novel tryptophan hydroxylase inhibitors for the potential treatment of functional gastrointestinal disorders. J Med Chem 2008;51:3684-7.

16. Young HM, Furness JB. Ultrastructural examination of the targets of serotoninimmunoreactive descending interneurons in the guinea pig small intestine. J Comp Neurol 1995;356:101-14.

17. Neal KB, Bornstein JC. Mapping 5-HT inputs to enteric neurons of the guinea-pig small intestine. Neuroscience 2007;145:556-67. 
18. Billett EE. Monoamine oxidase (MAO) in human peripheral tissues. Neurotoxicology 2004;25:139-48.

19. Kema IP, de Vries EG, Muskiet FA. Clinical chemistry of serotonin and metabolites. J Chromatogr B Biomed Sci Appl 2000;747:33-48.

20. Kohle C, Badary OA, Nill K, Bock-Hennig BS, Bock KW. Serotonin glucuronidation by Ah receptor- and oxidative stress-inducible human UDP-glucuronosyltransferase (UGT) 1 A6 in Caco-2 cells. Biochem Pharmacol 2005;69:1397-402.

21. Munzel PA, Bookjans G, Mehner G, Lehmkoster T, Bock KW. Tissue-specific 2,3,7,8tetrachlorodibenzo-p-dioxin-inducible expression of human UDPglucuronosyltransferase UGT1A6. Arch Biochem Biophys 1996;335:205-10.

22. Radominska-Pandya A, Little JM, Pandya JT, Tephly TR, King CD, Barone GW, Raufman JP. UDP-glucuronosyltransferases in human intestinal mucosa. Biochim Biophys Acta 1998;1394:199-208.

23. Squires LN, Jakubowski JA, Stuart JN, Rubakhin SS, Hatcher NG, Kim WS, Chen K, Shih JC, Seif I, Sweedler JV. Serotonin catabolism and the formation and fate of 5hydroxyindole thiazolidine carboxylic acid. J Biol Chem 2006;281:13463-70.

24. Mawe GM, Coates MD, Moses PL. Review article: intestinal serotonin signalling in irritable bowel syndrome. Aliment Pharmacol Ther 2006;23:1067-76.

25. van der Veek PP, Van Rood YR, Masclee AA. Symptom severity but not psychopathology predicts visceral hypersensitivity in irritable bowel syndrome. Clin Gastroenterol Hepatol 2008;6:321-8.

26. Ait-Belgnaoui A, Bradesi S, Fioramonti J, Theodorou V, Bueno L. Acute stressinduced hypersensitivity to colonic distension depends upon increase in paracellular permeability: role of myosin light chain kinase. Pain 2005;113:141-7.

27. Stone TW, Darlington LG. Endogenous kynurenines as targets for drug discovery and development. Nat Rev Drug Discov 2002;1:609-20.

28. Moffett JR, Namboodiri MA. Tryptophan and the immune response. Immunol Cell Biol 2003;81:247-65.

29. Yamazaki F, Kuroiwa T, Takikawa O, Kido R. Human indolylamine 2,3-dioxygenase. Its tissue distribution, and characterization of the placental enzyme. Biochem $\mathrm{J}$ 1985;230:635-8.

30. Wolf AM, Wolf D, Rumpold H, Moschen AR, Kaser A, Obrist P, Fuchs D, Brandacher G, Winkler C, Geboes K, Rutgeerts P, Tilg H. Overexpression of indoleamine 2,3dioxygenase in human inflammatory bowel disease. Clin Immunol 2004; 113:47-55.

31. Torres MI, Lopez-Casado MA, Lorite P, Rios A. Tryptophan metabolism and indoleamine 2,3-dioxygenase expression in coeliac disease. Clin Exp Immunol 2007; 148:419-24.

32. Ferdinande L, Demetter P, Perez-Novo C, Waeytens A, Taildeman J, Rottiers I, Rottiers P, De Vos M, Cuvelier CA. Inflamed intestinal mucosa features a specific epithelial expression pattern of indoleamine 2,3-dioxygenase. Int $\mathrm{J}$ Immunopathol Pharmacol 2008;21:289-95.

33. Uyttenhove C, Pilotte L, Theate I, Stroobant V, Colau D, Parmentier N, Boon T, Van den Eynde BJ. Evidence for a tumoral immune resistance mechanism based on tryptophan degradation by indoleamine 2,3-dioxygenase. Nat Med 2003;9:1269-74.

34. Kaszaki J, Palasthy Z, Erczes D, Racz A, Torday C, Varga G, Vecsei L, Boros M. 
Kynurenic acid inhibits intestinal hypermotility and xanthine oxidase activity during experimental colon obstruction in dogs. Neurogastroenterol Motil 2008;20:53-62.

35. Forrest CM, Gould SR, Darlington LG, Stone TW. Levels of purine, kynurenine and lipid peroxidation products in patients with inflammatory bowel disease. Adv Exp Med Biol 2003;527:395-400.

36. Kirchgessner AL. Glutamate in the enteric nervous system. Curr Opin Pharmacol 200 1;1:591-6.

37. Slattery JA, Page AJ, Dorian CL, Brierley SM, Blackshaw LA. Potentiation of mouse vagal afferent mechanosensitivity by ionotropic and metabotropic glutamate receptors. J Physiol 2006;577:295-306.

38. Hayaishi 0 . Properties and function of indoleamine 2,3-dioxygenase. J Biochem 1976;79:13P-21P.

39. Smith EA, Macfarlane GT. Formation of Phenolic and Indolic Compounds by Anaerobic Bacteria in the Human Large Intestine. Microb Ecol 1997;33:180-8.

40. Wikoff WR, Anfora AT, Liu J, Schultz PG, Lesley SA, Peters EC, Siuzdak G. Metabolomics analysis reveals large effects of gut microflora on mammalian blood metabolites. Proc Natl Acad Sci U S A 2009;106:3698-703.

41. Stott A, Khan M, Roberts C, Galpin IJ. Purple urine bag syndrome. Ann Clin Biochem 1987;24 ( Pt 2):185-8.

42. Marklova E. Where does indolylacrylic acid come from? Amino Acids 1999;17:40113.

43. Haverback BJ, Dyce B, Thomas HV. Indole metabolism in the malabsorption syndrome. N Engl J Med 1960;262:754-7.

44. Nowak A, Libudzisz Z. Influence of phenol, p-cresol and indole on growth and survival of intestinal lactic acid bacteria. Anaerobe 2006;12:80-4.

45. Bull G, Shattock P, Whiteley P, Anderson R, Groundwater PW, Lough JW, Lees G. Indolyl-3-acryloylglycine (IAG) is a putative diagnostic urinary marker for autism spectrum disorders. Med Sci Monit 2003;9:CR422-5.

46. D'Eufemia P, Celli M, Finocchiaro R, Pacifico L, Viozzi L, Zaccagnini M, Cardi E, Giardini 0. Abnormal intestinal permeability in children with autism. Acta Paediatr 1996;85:1076-9.

47. Wright B, Brzozowski AM, Calvert E, Farnworth H, Goodall DM, Holbrook I, Imrie G, Jordan J, Kelly A, Miles J, Smith R, Town J. Is the presence of urinary indolyl-3acryloylglycine associated with autism spectrum disorder? Dev Med Child Neurol 2005; $47: 190-2$.

48. Liebregts T, Adam B, Bredack C, Roth A, Heinzel S, Lester S, Downie-Doyle S, Smith E, Drew P, Talley NJ, Holtmann G. Immune activation in patients with irritable bowel syndrome. Gastroenterology 2007;132:913-20.

49. Fitzgerald P, Cassidy Eugene M, Clarke G, Scully P, Barry S, Quigley Eamonn MM, Shanahan F, Cryan J, Dinan Timothy G. Tryptophan catabolism in females with irritable bowel syndrome: relationship to interferon-gamma, severity of symptoms and psychiatric co-morbidity. Neurogastroenterol Motil 2008;20:1291-7.

50. Clarke G, Fitzgerald P, Cryan JF, Cassidy EM, Quigley EM, Dinan TG. Tryptophan degradation in irritable bowel syndrome: evidence of indoleamine 2,3-dioxygenase activation in a male cohort. BMC Gastroenterol 2009;9:6. 
51. Kim DY, Camilleri M. Serotonin: a mediator of the brain-gut connection. Am J Gastroenterol 2000;95:2698-709.

52. Gruninger TR, LeBoeuf B, Liu Y, Garcia LR. Molecular signaling involved in regulating feeding and other motivated behaviors. Mol Neurobiol 2007;35:1-20.

53. Shufflebotham J, Hood S, Hendry J, Hince DA, Morris K, Nutt D, Probert C, Potokar $J$. Acute tryptophan depletion alters gastrointestinal and anxiety symptoms in irritable bowel syndrome. Am J Gastroenterol 2006;101:2582-7.

54. Kilkens TO, Honig A, van Nieuwenhoven MA, Riedel WJ, Brummer RJ. Acute tryptophan depletion affects brain-gut responses in irritable bowel syndrome patients and controls. Gut 2004;53:1794-800.

55. Agazzi A, De Ponti F, De Giorgio R, Candura SM, Anselmi L, Cervio E, Di Nucci A, Tonini M. Review of the implications of dietary tryptophan intake in patients with irritable bowel syndrome and psychiatric disorders. Dig Liver Dis 2003;35:590-5.

56. Markus CR, Jonkman LM, Lammers JH, Deutz NE, Messer MH, Rigtering N. Evening intake of alpha-lactalbumin increases plasma tryptophan availability and improves morning alertness and brain measures of attention. Am J Clin Nutr 2005;81:102633.

57. Attenburrow MJ, Williams C, Odontiadis J, Powell J, Van de Ouderaa F, Williams M, Cowen PJ. The effect of a nutritional source of tryptophan on dieting-induced changes in brain 5-HT function. Psychol Med 2003;33:1381-6.

58. Miura H, Ozaki N, Sawada M, Isobe K, Ohta T, Nagatsu T. A link between stress and depression: shifts in the balance between the kynurenine and serotonin pathways of tryptophan metabolism and the etiology and pathophysiology of depression. Stress 2008;11:198-209.

59. Turski MP, Turska M, Zgrajka W, Kuc D, Turski WA. Presence of kynurenic acid in food and honeybee products. Amino Acids 2008. 
Tryptophan metabolites 
Does acute tryptophan depletion affect peripheral serotonin metabolism in the intestine?

D. Keszthelyi, FJ. Troost, DM. Jonkers, E. van Donkelaar, J. Dekker, WA. Buurman and AA. Masclee

American Journal of Clinical Nutrition 2012 Mar;95(3):603-8

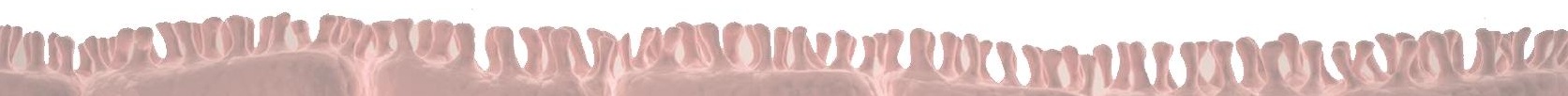




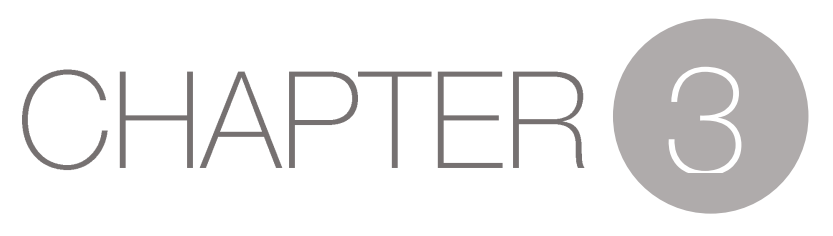

Acute tryptophan depletion

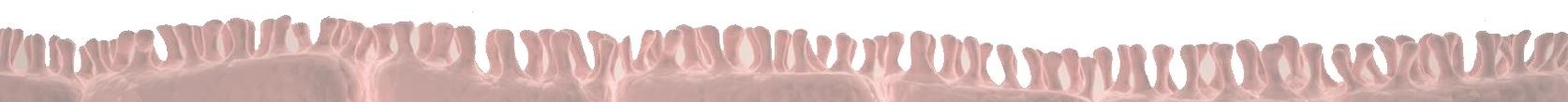




\section{ABSTRACT}

Background - Serotonin (5-hydroxytryptamine; 5-HT), a tryptophan metabolite, plays an important regulatory role in the human central nervous system and in the gastrointestinal tract. Acute tryptophan depletion (ATD) is currently the most widely established method to investigate $5-\mathrm{HT}$ metabolism.

Objective - Aim of this study was to assess the effect of an acute decrease in the systemic availability of the tryptophan on intestinal $5-\mathrm{HT}$ metabolism and permeability.

Design - 33 healthy volunteers (17 ATD of which 3 drop-outs, 16 placebo) participated in this randomized placebo-controlled study. Plasma and duodenal mucosal concentrations of $5-\mathrm{HT}, 5$-hydroxyindoleacetic acid and kynurenic acid were determined by HPLC-MS. Intestinal barrier function was assessed by a multi-sugar plasma test and analysis of tight junction transcription was performed in duodenal biopsies obtained by gastroduodenoscopy.

Results - Mucosal 5-HT, 5-HIAA and KA levels remained unaltered by ATD. In contrast, ATD significantly decreased plasma 5-HT ( $p<0.05)$ and 5-HIAA levels ( $p<0.0001)$. After endoscopy, a significant increase in 5-HT plasma concentrations was observed in the placebo group ( $p=0.029$ ) compared to ATD. Moreover, a significant increase in KA plasma levels over time was found in the placebo group ( $p<0.05)$. No changes in intestinal barrier function were observed.

Conclusion - An acute decrease in precursor availability does not affect mucosal concentrations of serotonergic metabolites, in contrast to systemic concentrations. ATD alters biochemical responses to acute stress of the endoscopic examination reflected by lower 5-HT concentrations. Changes in 5-HT concentrations were paralleled by alterations in KA concentrations, suggesting competition between the two metabolic pathways for the mutual precursor. 


\subsection{INTRODUCTION}

The amino acid tryptophan is precursor of a wide array of metabolites involved in a variety of aspects of human nutrition and metabolism. Studies in the past decades have primarily focused on the metabolite serotonin (5-hydroxytryptamine; 5-HT), a signaling molecule, which has been shown to play an important role in the human body, not only in the central nervous system (CNS), but also in the gut.., 2 Approximately $95 \%$ of total $5-\mathrm{HT}$-content of the human body is found in the gut of which $90 \%$ in the intestinal enterochromaffin (EC) cells. These cells possess the machinery to produce and store 5HT.2, 3 Besides its large mucosal source, $5-\mathrm{HT}$ is also produced by neurons of the enteric nervous system. In the gut, $5-\mathrm{HT}$ plays an important role in mediating gastrointestinal secretion, motility and perception, whereas in the central nervous system, it modulates an extensive range of physiological and behavioral functions including, mood, cognition and sleep. Besides incorporation into protein, only $1 \%$ of ingested tryptophan is converted into $5-\mathrm{HT}$, while the majority of tryptophan is subject to degradation via the kynurenine pathway, the primary route of tryptophan metabolism in the human body. ${ }^{4}$ Apart from 5$\mathrm{HT}$, metabolites of the kynurenine pathway have received increasing attention, as they are believed to have a regulatory role in the CNS and the gastrointestinal tract. ${ }^{4,5}$

The biology of the serotonergic system is often studied using protocols employing depletion of the precursor tryptophan. Acute tryptophan depletion (ATD) is a wellestablished dietary technique to temporarily reduce $5-\mathrm{HT}$ synthesis by decreasing the availability of tryptophan.6,7 ATD involves the ingestion of a pure amino acid mixture devoid of tryptophan which results in a 70-90\% decrease of plasma tryptophan and paralleled by lowering of the central 5-HT synthesis between 4 and $7 \mathrm{~h}$ of administration. ${ }^{6}$ Little is known of the consequences of tryptophan depletion on serotonergic functions outside the CNS. In this study, we aimed to investigate the intestinal mucosal serotonin metabolism and to assess its role in maintaining intestinal barrier function in healthy volunteers. The intestinal barrier plays a paramount role in regulating intestinal physiology and has been reported to be affected by $5-\mathrm{HT} .^{8}$ In order to influence serotonergic metabolism, we employed ATD. We aimed to test the general a priori 
hypotheses that (1) an acute decrease in the systemic availability of the precursor induces changes in serotonergic metabolism in the intestine and (2) that these changes in serotonergic conditions affect the intestinal barrier function. Furthermore, as the effects of precursor depletion on the kynurenine pathway have not been investigated previously, we aimed to assess effects of ATD on the systemic and intestinal mucosal concentrations of kynurenic acid, as an indicator of changes in the kynurenine pathway.

\subsection{SUBJECTS AND METHODS}

Approval for the study was obtained from the Medical Ethics Committee of the Maastricht University Medical Centre (MUMC) in full accordance with the principles of the Declaration of Helsinki (as amended in Tokyo, Venice, Hong Kong, Somerset West and Edinburgh. Note of clarification added in Washington and Tokyo). All volunteers gave their written informed consent prior to participation. The study has been registered in the US National Library of Medicine (http://www.clinicaltrials.gov, NCT00731003).

\section{SUBJECTS}

Participants eligible for inclusion were aged between 18 and 65 yrs. All subjects were screened by means of a standardized general physical examination. General psychological state was assessed using the 17 item Hamilton depression rating scale (HAM-D 17) and the Dutch version of the symptom checklist (SCL-90). Exclusion criteria included: history of gastrointestinal or psychiatric disorders including use of psychoactive medication or psychological symptomatology, defined as a diagnosis on the global severity index score on SCL-90 for females $\geq 150$, for males $\geq 131$, or HAM-D 17 scores $\geq 8$, first-degree family members with psychiatric disorders, use of medication, including vitamin supplementation, except oral contraceptives, within 14 days prior to testing, administration of investigational drugs in the 180 days prior to the study, major abdominal surgery interfering with gastrointestinal function, premenstrual syndrome, dieting (medically prescribed, vegetarian, diabetic, macrobiological, biological dynamic), pregnancy, lactation, excessive alcohol consumption ( $>20$ alcoholic consumptions per week) and smoking. 


\section{ACUTE TRYPTOPHAN DEPLETION}

The amino acid mixtures were obtained from Tiofarma BV, Oud Beijerland, the Netherlands, and blinded by the Department of Pharmacy of the MUMC, Maastricht, the Netherlands. The depleting drink contained 15 amino acids, including five large neutral amino acids, according to the formula of Young et al. ${ }^{9}$ The control mixture contained the same amino acids enriched with $2.3 \mathrm{~g}$ of tryptophan in order to maintain normal tryptophan levels.

\section{STUDY DESIGN}

The study was conducted following a randomized, placebo-controlled, double blind, parallel design and consisted of one test day. All women were tested in the follicular phase of the menstrual cycle or while taking oral contraception. All subjects were tested within 3 months to avoid potential seasonal variation. Participants were requested to abstain from heavy physical exercise and consumption of tryptophan-rich food (coffee, tea, energy drinks, kiwi, avocado, fish, sea food, walnut, banana, tomato) the day prior to their visit. After 10:00 pm, no eating or drinking (except water) was allowed.

Figure 1 gives an overview of the experimental procedures on the test day. Participants were requested to arrive at the MUMC at 8:00 AM after which they received an intravenous cannula in the antecubital vein. After taking blood samples, the amino acid mixtures were dissolved in $300 \mathrm{~mL}$ tap water and subjects were instructed to ingest the mixture as quickly as possible using a nose clap to avoid the unpleasant smell of the mixtures. Four hours later, a venous blood sample was taken and participants received an oral multi-sugar drink to assess intestinal permeability. The multi-sugar drink consisted of $1 \mathrm{~g}$ lactulose (Centrafarm Services, Etten-Leur, the Netherlands), $0.5 \mathrm{~g}$ L-rhamnose (Danisco Sweeteners, Thomson, IL), $1 \mathrm{~g}$ erythritol (Cargill Nederland BV, the Netherlands) and $1 \mathrm{~g}$ sucralose (Tate and Lyle Sucralose Inc., Reading, UK). An additional blood sample was taken $5 \mathrm{~h}$ following ingestion of the amino acid drink. Subsequently, participants underwent a gastroduodenoscopy and mucosal tissue samples of approximately $5 \mathrm{mg}$ were obtained from the $2^{\text {nd }}$ segment of the duodenum using a standard forceps (diameter 
$2.8 \mathrm{~mm}$ ) and immediately frozen in liquid nitrogen. At the end of the test day, a blood sample was drawn at $\mathrm{t}=330$ min following ingestion of the amino acid drink.

\section{Test day for intestinal permeability}

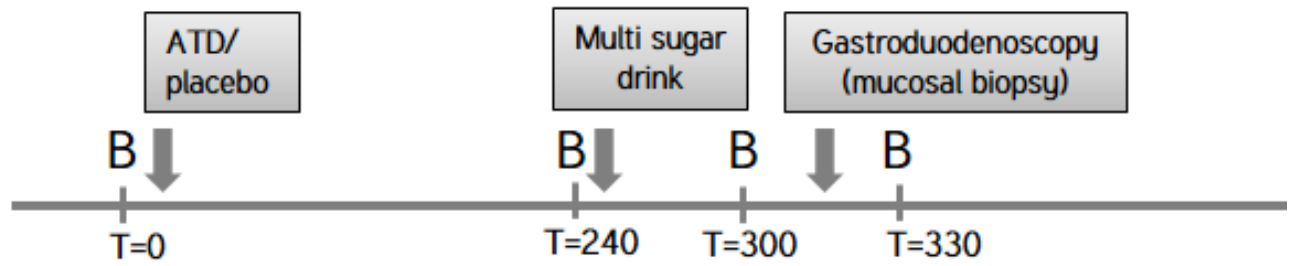

Figure 1: Timeline of the test day, $\mathrm{B}=$ blood sampling. T= time in minutes.

\section{ASSESSMENT OF SEROTONERGIC METABOLISM}

To assess systemic concentrations of 5-HT metabolites, blood samples were collected using pre-chilled K2EDTA tubes. One ml 1.4\% ascorbic acid (Sigma Aldrich, St. Louis, MO) was added to all tubes for $5-\mathrm{HT}$ analysis to prevent oxidative breakdown. Plasma collection tubes were centrifuged at $2000 \mathrm{~g}$ at $4^{\circ} \mathrm{C}$ for 10 minutes to obtain platelet poor plasma. Supernatants were collected and stored at $-80^{\circ} \mathrm{C}$ until analysis. Concentrations of 5-HT, 5-hydroxy-indoleacetic acid (5-HIAA, the main metabolite of 5-HT) and kynurenic acid (a metabolic product of the kynurenine pathway) were determined by highperformance liquid chromatography-mass spectrometry tandem (HPLC-MS) as described by Danaceau et al. ${ }^{10}$

Mucosal concentrations of 5-HT, 5-HIAA and KA were determined using the same method following measurement of the wet weight of tissue samples. Furthermore, the gene expression of the proteins serotonin transporter (SERT, responsible for mucosal clearance of 5-HT released from EC cells) and tryptophan hydroxylase 1 (TPH1, the rate limiting step in 5-HT synthesis in EC cells) were investigated in mucosal biopsy specimens by qRT-PCR (see supplementary material). 


\section{ASSESSMENT OF INTESTINAL BARRIER FUNCTION}

Intestinal barrier function was assessed by employing the sugar recovery test. Measuring the plasma levels of orally ingested sugar is a generally accepted method to investigate intestinal permeability. ${ }^{11}$ The ratio of a monosaccharide and a disaccharide is indicative of intestinal mucosal permeability, as these probes differ in manner of transport, i.e. paracellular or transcellular. In the healthy small bowel, the permeability for larger sugars such as lactulose or sucralose is much lower than for smaller sugars such as erythritol or rhamnose. Lactulose and other larger molecules pass through the intercellular spaces, which are regulated by intercellular tight junctions. ${ }^{11}$ Measurements of plasma sugar concentrations was performed as described by van Wijck et al. ${ }^{12}$

Furthermore, gene transcription of tight junction proteins (occludin, claudin-3, claudin-4 and zonuline occludens-1) and myosin light chain kinase (MLCK, involved in regulating tight junction function) in biopsy specimens was determined by qRT-PCR. Glyceraldehyde 3-phosphate dehydrogenase (GAPDH) and 18S rRNA were used as reference genes.

\section{ASSESSMENT OF THE STRESS RESPONSE TO THE ENDOSCOPIC EXAMINATION}

In order to assess the magnitude of the stress response to the endoscopic examination, serum was collected before and after the endoscopy to measure cortisol concentrations. Serum collection tubes were stored at room temperature for $1 \mathrm{~h}$ and centrifuged at 3000 $\mathrm{g}$ at $4^{\circ} \mathrm{C}$ for $10 \mathrm{~min}$. Serum cortisol concentrations were analyzed by ELISA at Medische Laboratoria Dr. Stein \& Collegae (Maastricht, the Netherlands).

\section{STATISTICAL ANALYSES}

Statistical analyses were performed using SPSS 17.0 for windows (SPSS Inc. Chicago IL). Data was tested for normality by the Kolmogorov-Smirnoff test. Normally distributed data were analyzed by independent Student's $t$ test. Wilcoxon and Mann-Whitney U-test were used in other cases. Coefficients for correlations were calculated according to Pearson. Alterations in plasma concentrations of serotonergic metabolites induced by ATD over 


\section{Chapter 3}

time were also analyzed using a 2-factor ANOVA with interaction. Data are expressed as mean \pm SEM.

\subsection{RESULTS}

\section{STUDY SUBJECT}

Thirty-three healthy volunteers ( $33 \%$ male; age $26 \pm 2$ years; $\mathrm{BMl} 24 \pm 1 \mathrm{~kg} / \mathrm{m}^{2}$ ) participated in this study. Seventeen participants received an amino acid drink devoid of the 5-HT precursor tryptophan (ATD), sixteen received the same drink to which tryptophan was added (placebo). Three participants in the ATD-group dropped out due to side-effects (nausea and vomiting following ingestion of the drink).

\section{EFFECT OF ATD ON MUCOSAL 5-HT METABOLITES}

Mucosal 5-HT, 5-HIAA and KA concentrations were not altered significantly by ATD (Table 1). Furthermore, ATD did not induce changes in the transcription of enzymes involved in serotonergic metabolism, TPH1 or SERT (see Table 2).

\begin{tabular}{l|lll}
\multicolumn{1}{c}{ ATD } & PLACEBO & P-VALUE \\
\hline $5-H T$ & $45.6 \pm 7.3$ & $42.5 \pm 6.7$ & 0.76 \\
$5-H I A A$ & $1.57 \pm 0.3$ & $1.97 \pm 0.3$ & 0.36 \\
KA & $2.07 \pm 1.3$ & $2.29 \pm 1.3$ & 0.91
\end{tabular}

Table 1: Concentrations of 5-hydroxytryptamin (5-HT), 5-hydroxyindoleacetic acid (5HIAA) and kynurenic acid (KA) in duodenal mucosal samples (pmol/mg).

\begin{tabular}{l|lll}
\multicolumn{1}{c}{} & \multicolumn{1}{c}{ ATD } & \multicolumn{1}{c}{ PLACEBO } & \multicolumn{1}{c}{ P-VALUE } \\
\hline TPH1 & $0.0006 \pm 0.0002$ & $0.0004 \pm 0.0001$ & 0.399 \\
SERT & $0.99 \pm 0.35$ & $0.57 \pm 0.20$ & 0.413 \\
CLAUDIN-3 & $1.64 \pm 0.49$ & $1.42 \pm 0.50$ & 0.764 \\
CLAUDIN-4 & $2.02 \pm 0.65$ & $1.75 \pm 0.49$ & 0.741 \\
ZO-1 & $0.58 \pm 0.19$ & $0.79 \pm 0.18$ & 0.446
\end{tabular}



OCCLUDIN
$1.04 \pm 0.35$
$0.76 \pm 0.20$
0.496
MLCK
$1.52 \pm 0.34$
$1.01 \pm 0.25$
0.237

Table 2: Gene transcription of genes associated with serotonergic metabolism and intestinal barrier function.

\section{EFFECT OF ATD ON SYSTEMIC 5-HT AND KYNURENINE METABOLITES}

ATD significantly decreased plasma $5-\mathrm{HT}$ levels at $5 \mathrm{~h}$ after ingestion ( $10.5 \pm 3$ vs $21.8 \pm 6$ nmoVl, ATD vs placebo; $p<0.05$, see Figure $2 A$ ). The two-way ANOVA revealed a significant interaction between treatment and time $(p<0.01)$. Also, the levels of the principal metabolite 5-HIAA were significantly decreased following ATD $(11.7 \pm 1.2$ vs $22.7 \pm 1.8 \mathrm{nmoV} /$, ATD vs placebo; $p<0.0001$, see Figure 2B). Two-way ANOVA showed significant interaction between treatment and time ( $p<0.001)$. Kynurenic acid levels remained unaltered in the ATD group, whereas a significant increase over time was observed in the placebo group ( $6.5 \pm 1.4$ vs $14.2 \pm 3.3$ nmoVl; $t=0$ vs $t=240 \mathrm{~min}, p<0.05$, see Figure $2 \mathrm{C}$ ). Two-way ANOVA showed a significant interaction between treatment and treatment $(p=0.03)$.

\section{EFFECT OF ATD ON INTESTINAL PERMEABILITY}

ATD did not affect the plasma levels of the sugars lactulose, rhamnose, sucralose and erythritol. Therefore, no significant differences were found in lactulose/rhamnose or sucralose/erythritol ratios (Table ). Furthermore, ATD did not lead to any changes in the transcription of the tight junction proteins claudin-3, claudin-4, zonula occludens-1, occludin as well as MLCK (Table).

\begin{tabular}{l|ccl}
\multicolumn{1}{c}{ ATD } & PLACEBO & P-VALUE \\
\hline LACTULOSE/RHAMNOSE & $0.0017 \pm 0.001$ & $0.0021 \pm 0.001$ & 0.434 \\
RATIO & \\
SUCRALOSE/ERYTHRITOL & $0.0044 \pm 0.001$ & $0.0042 \pm 0.001$ & 0.540 \\
RATIO & &
\end{tabular}

Table 3: Plasma sugar ratios measured 1 hour after administration of the multi-sugar drink. 

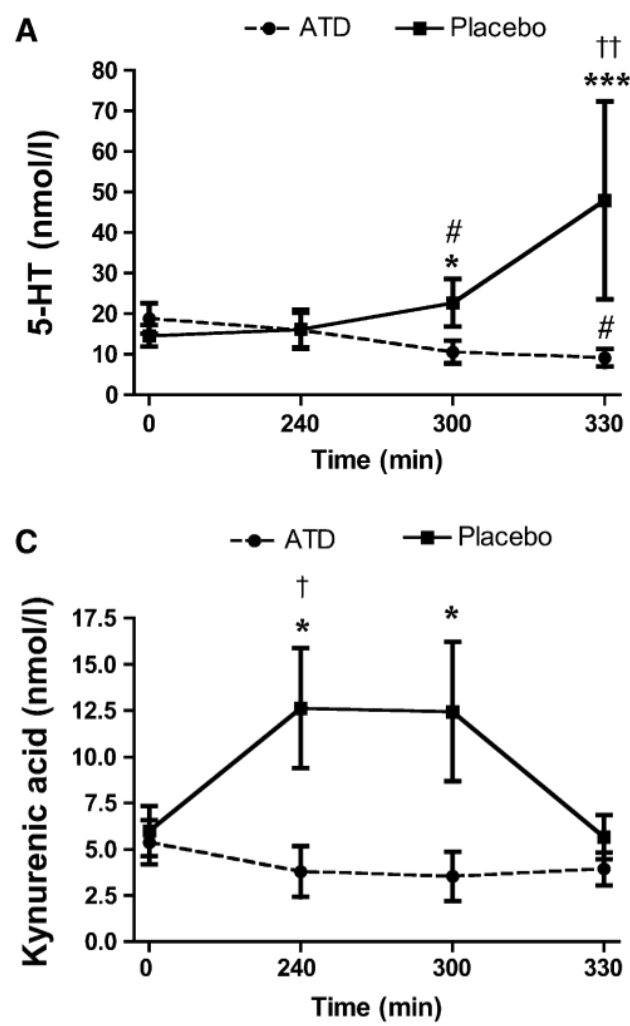

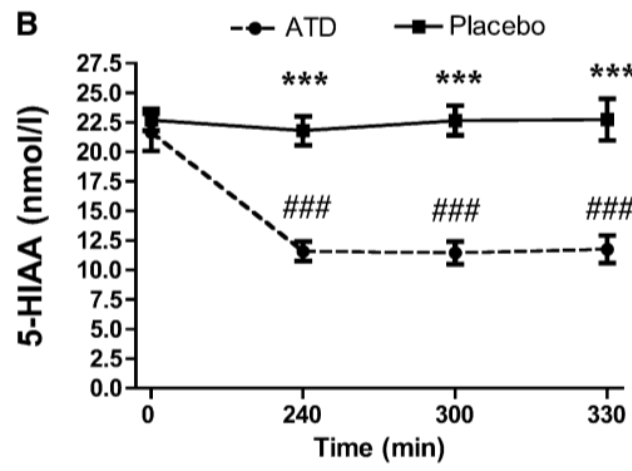

Figure 2: Platelet poor plasma concentrations of 5-hydroxytryptamine (5HT), 5-hydroxyindoleacetic acid (5-HIAA) and kynurenic acid (nmol/l). Data expressed as mean \pm SEM. Data analyzed using twoway ANOVA and Student's t test. $P$ values for interaction $p<0.01, p$

\section{EFFECT OF ACUTE STRESS RESPONSE ON 5-HT AND KYNURENINE METABOLITES}

Serum cortisol concentrations increased significantly following endoscopic examination (from $11.9 \pm 1.6$ to $15.8 \pm 1.3 \mu \mathrm{g} / \mathrm{dl} ; \mathrm{p}=0.001$; before vs. after endoscopy). The increase in cortisol concentrations did not differ significantly between the ATD and placebo group, indicating that the acute stress response as reflected by the increased serum cortisol concentrations was independent of ATD condition (increase corrected for initial cortisol concentrations $3.1 \pm 1.8$ to $4.4 \pm 1.1 \mu \mathrm{g} / \mathrm{dl} ; \mathrm{p}=0.476$, placebo vs ATD). This finding is in line with earlier studies demonstrating that ATD does not influence cortisol release. ${ }^{13,} 14$ Interestingly, a significant increase in plasma 5-HT concentrations was observed in the placebo group following the endoscopy ( $21.8 \pm 6$ vs $48 \pm 24 \mathrm{nmoV} / \mathrm{p} p=0.029$; before vs after endoscopy). This increase between pre-and post-endoscopy concentrations correlated significantly with the increase in serum cortisol levels observed over the same period of 
time ( $r=0.879, p=0.02)$. The observed $5-H \mathrm{~T}$ increase following endoscopy remained absent in the ATD group (see Figure 2A). At the same time, KA concentrations decreased to their initial level following the endoscopy in the placebo group, whereas no significant alterations in KA concentrations were seen in the ATD group.

\subsection{DISCUSSION}

Decreasing tryptophan availability by ATD has been demonstrated to decrease brain 5HT synthesis throughout much of the cerebral cortex in healthy volunteers. ${ }^{15}$ As tryptophan hydroxylase, the rate limiting synthesis of $5-\mathrm{HT}$, is half saturated under normal physiological conditions, ${ }^{16} 5-\mathrm{HT}$ synthesis in the brain is highly dependent on the availability of tryptophan in plasma, which is largely influenced by a competitive uptake of tryptophan and other large neutral amino acids across the blood brain barrier through the large neutral amino acid transporter 1 (LAT1). ${ }^{17}$ Next to the CNS, the gut is a principle organ of 5-HT synthesis and metabolism. A similar competitive uptake is theoretically also possible for the carrier responsible for the intestinal absorption of tryptophan and all other neutral amino acids, $\mathrm{B}^{0} \mathrm{AT} 1$, which has a relatively low affinity for tryptophan. ${ }^{4}$ This could provide a basis for decreased uptake of tryptophan through the intestinal epithelium following ATD, which could potentially affect intestinal 5-HT metabolism. Here we investigated whether an acute decrease in tryptophan availability can affect intestinal mucosal 5-HT metabolism and in particular in the enterochromaffin cells, the primary site of 5 -HT synthesis in human.

This study suggests that the machinery responsible for maintaining serotonergic homeostasis in the intestinal mucosa has the capacity to compensate for an acute decrease in the precursor availability thereby leaving intestinal 5-HT concentrations unaffected. In support of this, we found no alterations in the duodenal mucosal concentrations of serotonergic metabolites during ATD. Neither were changes in the mucosal expression of genes related to intestinal serotonergic metabolism observed. Additionally, the intestinal barrier function was investigated. In a recent study we were demonstrated that oral administration of the precursor of 5-HT, 5-hydroxytryptophan, 
reinforces small intestinal barrier function by lowering intestinal sugar permeability, inducing the expression of the tight junction protein ZO-1 and causing alterations in the intracellular distribution of tight junction proteins. This suggests that changes in serotonergic conditions can potentially influence intestinal barrier function. ${ }^{18}$ However, in the current study employing ATD, neither the expression of genes related to intestinal barrier function, nor plasma levels of orally ingested sugars, indicative of altered intestinal permeability, were affected. Furthermore, the current findings are supported by a recent study suggesting that an acute decrease of precursor availability is unlikely to interfere with serotonergic metabolism in EC cells of the mucosa. ${ }^{19}$ These observations are in line with the fact that maintenance of steady-state mucosal 5-HT concentrations is essential for the orchestration of intestinal function.

The fact that a decrease in precursor availability was indeed achieved by ATD in our study was reflected by profound alterations observed in systemic levels of 5-HT metabolites induced by ATD: plasma concentrations of 5-HT and 5-HIAA, the main metabolic product of $5-\mathrm{HT}$, had decreased significantly 5 hours after ATD in comparison to the placebo drink. Earlier studies have demonstrated that ATD induces a significant decrease in both plasma and cerebrospinal fluid (CSF) concentrations of 5-HIAA. ${ }^{19-22}$ CSF $5-\mathrm{HIAA}$ is thought to reflect central 5-HT turnover and presumed to correlate with brain 5 -HIAA in man, ${ }^{23}$ supporting the hypothesis that ATD affects serotonergic conditions in the CNS. Given the lack of effect on mucosal serotonergic metabolism, it is tempting to assume that alterations seen in systemic levels of 5-HT metabolites originate from changes induced in other than mucosal sources of 5-HT. As 5-HT synthesis in the CNS is highly dependent on plasma tryptophan availability, we postulate that alterations observed in systemic 5-HT levels following precursor depletion originate from changes in the CNS.

The fact that precursor availability strongly affects 5-HT synthesis in the CNS was reflected by an altered reaction to a stress response. In this study, we carried out gastroduodenoscopies to obtain biopsy specimens from the duodenal mucosa. 
Interestingly, we observed an unanticipated, significant increase in plasma 5-HT concentrations in the placebo group following the endoscopic procedure, which was absent in the ATD group. We postulate that the increase was caused by an acute stress response related to the endoscopic examination, and is not the result of normal plasma 5-HT kinetics following tryptophan administration, as an earlier study showed no significant change in plasma 5-HT up to 7 hours following intake of the amino acid drink. ${ }^{20}$ Acute stress and anxiety have been associated with increased plasma concentrations of $5-\mathrm{HT}$, which has been postulated to be the result of impaired uptake of 5-HT into platelets due to increased adrenergic activity. ${ }^{24}$ In order to assess potential stress or anticipatory anxiety, we measured serum cortisol concentrations that generally increase following acute stress due to HPA-axis activation. Both groups showed a significant increase in serum cortisol levels, supporting an acute stress response. The absence of an increase in 5-HT concentrations in the ATD group is likely to be the result of precursor depletion, also supporting the hypothesis that alterations observed in systemic serotonergic metabolite concentrations induced by ATD are driven centrally.

A possible limitation of our study is the fact that the time window of 5.5 hours might have been suboptimal with regards to detecting changes in intestinal 5-HT metabolism. Although Geeraerts et al. demonstrated an absolute decrease in duodenal 5-HT content, ${ }^{19}$ there is currently insufficient evidence to determine the optimal time window to assess changes in intestinal 5-HT metabolism. Furthermore, Geeraerts et al. observed modest effects on gastric physiology 5 hours following ATD, with no alterations in gastric sensitivity and compliance. Therefore, the profound alterations observed in systemic 5HT concentrations, the relative stability of plasma 5-HIAA levels within the time period examined, as well as the modest effects on functional outcomes from both the current study as well as the study by Geeraerts et al., all point to the fact that no profound effects can be observed in the intestine following ATD.

In this study, we also investigated the effects of a decrease in tryptophan availability on the kynurenine pathway. The vast majority (95\%) of ingested tryptophan is subject to 
metabolism along this pathway. ${ }^{4}$ Plasma tryptophan levels are largely under control of the liver by clearing any excess tryptophan through tryptophan dioxygenase, the initiating enzyme of the kynurenine pathway. ${ }^{4}$ Tryptophan, which was administered in the placebo but not in the ATD group, was therefore subject to conversion along the kynurenine pathway, which was reflected by significantly increased plasma concentrations of KA. This is in line with findings from early studies that employed tryptophan loading and demonstrated an increase in urinary KA concentrations. ${ }^{25}$ On the other hand, synthesis of KA apparently was independent of plasma precursor levels, as plasma and intestinal mucosal KA concentrations remained unaltered in the depleted group. Additionally, we observed that alterations in the plasma concentrations of KA were counterbalanced by changes in plasma concentrations of $5-\mathrm{HT}$. As a result of the acute stress response, the initially increased KA concentrations observed in the placebo group dropped to baseline, whereas 5-HT concentrations increased significantly. This suggests that the two metabolic pathways (5-HT and kynurenine) compete for their mutual precursor, tryptophan, leading to lower concentrations of KA upon increased 5-HT synthesis. Vice versa, diversion of tryptophan to the kynurenine pathway may potentially lead to a relative deficiency of $5-\mathrm{HT}$ synthesis and hence serotonergic dysfunction. ${ }^{26}$ This is supported by previous reports suggesting that such metabolic imbalance plays a role in the development of disorders associated with serotonergic dysfunction in both CNS and periphery, such as mood disorder and irritable bowel syndrome.4, 27

\subsection{CONCLUSION}

In this study, we demonstrated that the serotonergic metabolism in the intestinal mucosa was not affected by an acute dietary decrease in precursor availability, whereas profound effects on systemic concentrations of serotonergic metabolites were observed. We also show that precursor depletion alters responses to acute stress, as systemic 5-HT concentrations did not increase in the ATD group due to depletion of the precursor. Furthermore, changes in plasma 5-HT concentrations were paralleled by alterations in systemic concentrations of $\mathrm{KA}$, suggesting competition between the two metabolic 
pathways for the mutual precursor. Findings of this study contribute to a more complete understanding of $5-\mathrm{HT}$ and kynurenine metabolism and provides further basis for the development of novel therapeutic entities for disorders associated with serotonergic dysfunction. 


\subsection{REFERENCES}

1. Beattie DT, Smith JA. Serotonin pharmacology in the gastrointestinal tract: a review. Naunyn Schmiedebergs Arch Pharmacol 2008;377:181-203.

2. Gershon MD, Tack J. The serotonin signaling system: from basic understanding to drug development for functional GI disorders. Gastroenterology 2007;132:397-414.

3. Lesurtel M, Soll C, Graf R, Clavien PA. Role of serotonin in the hepatogastrolntestinal tract: an old molecule for new perspectives. Cell Mol Life Sci 2008;65:940-52.

4. Keszthelyi D, Troost FJ, Masclee AA. Understanding the role of tryptophan and serotonin metabolism in gastrointestinal function. Neurogastroenterol Motil 2009;21:1239-49.

5. Stone TW. Kynurenines in the CNS: from endogenous obscurity to therapeutic importance. Prog Neurobiol 2001;64:185-218.

6. Bell C, Abrams J, Nutt D. Tryptophan depletion and its implications for psychiatry. Br J Psychiatry 200 1;178:399-405.

7. van Donkelaar EL, Blokland A, Ferrington L, Kelly PA, Steinbusch HW, Prickaerts J. Mechanism of acute tryptophan depletion: is it only serotonin? Mol Psychiatry 2011;16:695-713.

8. Haub S, Kanuri G, Volynets V, Brune T, Bischoff SC, Bergheim I. Serotonin reuptake transporter (SERT) plays a critical role in the onset of fructose-induced hepatic steatosis in mice. Am J Physiol Gastrointest Liver Physiol 20 10;298:G335-44.

9. Young SN, Smith SE, Pihl RO, Ervin FR. Tryptophan depletion causes a rapid lowering of mood in normal males. Psychopharmacology (Berl) 1985;87:173-7.

10. Danaceau JP, Anderson GM, McMahon WM, Crouch DJ. A liquid chromatographictandem mass spectrometric method for the analysis of serotonin and related indoles in human whole blood. J Anal Toxicol 2003;27:440-4.

11. Bjarnason I, MacPherson A, Hollander D. Intestinal permeability: an overview. Gastroenterology 1995; 108:1566-81.

12. van Wijck K, van Eijk HM, Buurman WA, Dejong $\mathrm{CH}$, Lenaerts K. Novel analytical approach to a multi-sugar whole gut permeability assay. J Chromatogr B Analyt Technol Biomed Life Sci 2011;879:2794-801.

13. Sobczak S, Honig A, Nicolson NA, Riedel WJ. Effects of acute tryptophan depletion on mood and cortisol release in first-degree relatives of type I and type II bipolar patients and healthy matched controls. Neuropsychopharmacology 2002;27:83442.

14. Porter RJ, Gallagher P, O'Brien JT. Effects of rapid tryptophan depletion on salivary cortisol in older people recovered from depression, and the healthy elderly. $J$ Psychopharmacol 2007;21:71-5.

15. Nishizawa S, Benkelfat C, Young SN, Leyton M, Mzengeza S, de Montigny C, Blier P, Diksic M. Differences between males and females in rates of serotonin synthesis in human brain. Proc Natl Acad Sci U S A 1997;94:5308-13.

16. Young SN, Gauthier S. Effect of tryptophan administration on tryptophan, 5hydroxyindoleacetic acid and indoleacetic acid in human lumbar and cisternal cerebrospinal fluid. J Neurol Neurosurg Psychiatry 1981;44:323-8. 
17. Fernstrom JD. Role of precursor availability in control of monoamine biosynthesis in brain. Physiol Rev 1983;63:484-546.

18. Keszthelyi D, Troost F, van Eijk H, Schaepkens E, Lindsey PJ, Jonkers D, Buurman WA, Dekker J, Masclee A. The Serotonin Precursor 5-Hydroxytryptophan Reinforces Intestinal Barrier Function. Gastroenterology 2011;140:S370-S370.

19. Geeraerts B, Van Oudenhove L, Boesmans W, Vos R, Vanden Berghe P, Tack J. Influence of acute tryptophan depletion on gastric sensorimotor function in humans. Am J Physiol Gastrointest Liver Physiol 2011;300:G228-35.

20. Kilkens TO, Honig A, van Nieuwenhoven MA, Riedel WJ, Brummer RJ. Acute tryptophan depletion affects brain-gut responses in irritable bowel syndrome patients and controls. Gut 2004;53:1794-800.

21. Moreno FA, McGavin C, Malan TP, Gelenberg AJ, Heninger GR, Mathe AA, Delgado PL. Tryptophan depletion selectively reduces CSF 5-HT metabolites in healthy young men: results from single lumbar puncture sampling technique. Int $\mathrm{J}$ Neuropsychopharmacol 2000;3:277-283.

22. Williams WA, Shoaf SE, Hommer D, Rawlings R, Linnoila M. Effects of acute tryptophan depletion on plasma and cerebrospinal fluid tryptophan and 5hydroxyindoleacetic acid in normal volunteers. J Neurochem 1999;72:1641-7.

23. Stanley M, Traskman-Bendz L, Dorovini-Zis K. Correlations between aminergic metabolites simultaneously obtained from human CSF and brain. Life Sci 1985;37:1279-86.

24. Naesh 0 , Hindberg I, Bruun AB. Decreased reuptake of serotonin in human platelets after surgery. Clin Physiol 200 1;21:39-43.

25. Leklem JE. Quantitative aspects of tryptophan metabolism in humans and other species: a review. Am J Clin Nutr 1971;24:659-72.

26. Laugeray A, Launay JM, Callebert J, Surget A, Belzung C, Barone PR. Evidence for a key role of the peripheral kynurenine pathway in the modulation of anxiety- and depression-like behaviours in mice: focus on individual differences. Pharmacol Biochem Behav 2011;98:161-8.

27. Miura H, Ozaki N, Sawada M, Isobe K, Ohta T, Nagatsu T. A link between stress and depression: shifts in the balance between the kynurenine and serotonin pathways of tryptophan metabolism and the etiology and pathophysiology of depression. Stress 2008;11:198-209. 
Decreased levels of kynurenic acid in the intestinal mucosa of IBS patients: relation to serotonin and psychological state

D. Keszthelyi, FJ. Troost, DM. Jonkers, JW. Kruimel, C. Leue, AAM. Masclee

Journal of Psychosomatic Research 2013 Jun;74(6):501-4

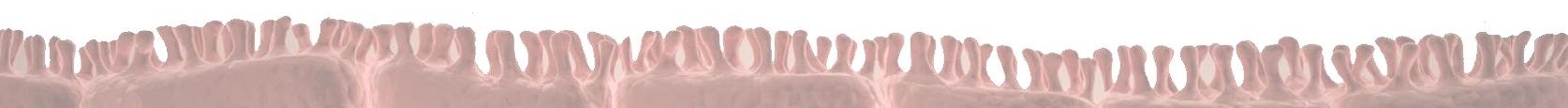




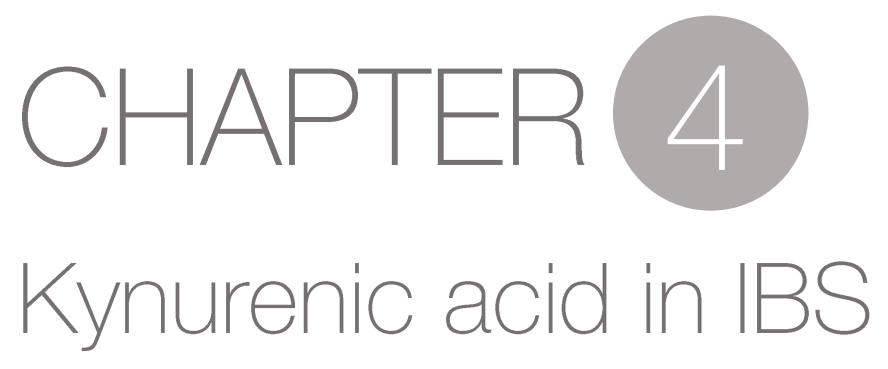

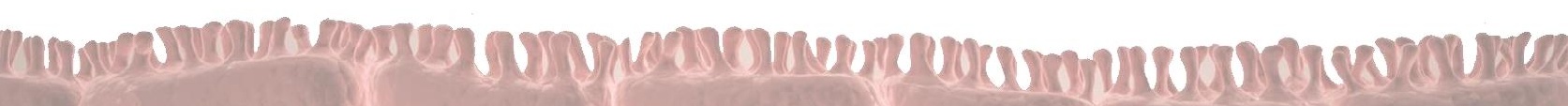




\section{ABSTRACT}

Objective: Irritable bowel syndrome (IBS) has been associated with psychiatric comorbidity and alterations in serotonergic metabolism. Tryptophan is the precursor of serotonin (5-HT), but it is mainly catabolized through the kynurenine pathway. This pathway may also be involved in the pathogenesis of IBS by virtue of deviating tryptophan from the 5-HT pathway resulting in 5-HT deficiency. We therefore aimed to ascertain the mucosal and systemic concentrations of 5-HT and kynurenic acid (KYNA), a principle kynurenine metabolite.

Methods: Duodenal mucosal biopsy specimens and platelet poor plasma samples were obtained from 15 healthy volunteers and 15 IBS patients. Psychological state was assessed using the Hospital Anxiety and Depression Scale and the 90-item symptom checklist.

Results: IBS patients showed significantly lower mucosal and higher systemic concentrations of both 5-HT and KYNA compared to healthy controls. Also, significant correlation between mucosal but not plasma concentrations of KYNA and 5-HT and psychological state in IBS was observed.

Conclusion: The observation that mucosal KYNA and 5-HT are both decreased in IBS does not support the hypothesis that increased activation along the kynurenic pathway results in relative 5-HT deficiency. However, an increased release of these substances from the intestine to the systemic compartment may lead to a decrease in intestinal KYNA and 5-HT levels, resulting in disturbance of intestinal homeostasis. Thus, changes in psychological states observed in IBS patients may be secondary to alterations in gastrointestinal function, and in particular kynurenine and/or 5-HT metabolism. 


\subsection{INTRODUCTION}

Irritable bowel syndrome (IBS) is a functional gastrointestinal disorder affecting up to $15 \%$ of the Western population. Despite being very common, the pathophysiology of IBS is incompletely understood.' Several studies have provided evidence that IBS is associated with a dysregulation of the brain-gut axis - a theoretical concept describing a bidirectional connection, of which serotonin (5-hydroxytryptamine, 5-HT) appears to be an important modulator. ${ }^{2}$ In the periphery, 5-HT is involved in mediating gastrointestinal secretion, motility and perception, whereas in the central nervous system (CNS), 5-HT modulates an extensive range of physiological and behavioral processes. ${ }^{3}$ - 5 T is derived from tryptophan. Merely 1\% of ingested tryptophan is converted into 5-HT, while the majority is catabolized via the kynurenine pathway, the primary route of tryptophan metabolism in the human body. ${ }^{4}$ Metabolites of the kynurenine pathway have recently received increasing attention, as they are also believed to have a regulatory role in both the CNS and the gastrointestinal tract. ${ }^{4,5}$

We have recently demonstrated that these two metabolic pathways (5-HT and kynurenine) compete for their mutual precursor tryptophan, in particular following acute stress. ${ }^{6}$ This implies that diversion of tryptophan to the kynurenine pathway may potentially lead to a relative deficiency of $5-\mathrm{HT}$ synthesis and hence serotonergic dysfunction. This concept of pathway imbalance has been suggested as a factor involved in the development of disorders associated with serotonergic dysfunction in both CNS and periphery.4, 7 Such a metabolic dysfunction may also provide a mechanistic link between IBS and its high co-morbidity with psychiatric conditions such as affective and anxiety disorders. Aim of this study was therefore three-fold. First, we aimed to assess the mucosal and systemic concentration of kynurenic acid (KYNA), a principle kynurenine metabolite, suggested to play a role in gastrointestinal function, ${ }^{8}$ in IBS patients compared to healthy controls. Second, we aimed to assess the mucosal and systemic concentration of $5-\mathrm{HT}$, in order to ascertain the relationship between the two pathways. Third, we aimed to ascertain the association between potential alterations in 5-HT and KYNA concentrations to psychological state. 


\subsection{METHODS}

This study was part of a larger project investigating the role of 5-HT in IBS approved by the Medical Ethics Committee of the Maastricht University Medical Centre (MUMC) and was conducted according to the revised version of the Declaration of Helsinki (October 2008, Seoul). All participants gave their written informed consent prior to participation. The study has been registered at the US National Library of Medicine (http://www.clinicaltrials.gov, NCT00731003).

\section{PARTICIPANTS}

Fifteen healthy volunteers and 15 patients with IBS diagnosed according to the Rome III criteria were included. Exclusion criteria included history of gastrointestinal disorders other than IBS; use of psychoactive medication (including serotonin reuptake inhibitors); use of medication influencing gastrointestinal function within 14 days prior to testing. During the screening period, psychological state was assessed using the 17-item Hospital Anxiety and Depression Scale (HADS) and the Symptom Checklist-90 (SCL-90). Global severity index on the SCL-90 was used to assess general psychological state.

\section{STUDY PROCEDURES}

Participants arrived at the MUMC after an overnight fast at 8:00 AM. Blood samples were drawn from the antecubital vein. Hereafter, at 10:00 AM, participants underwent a gastroduodenoscopy, during which mucosal samples from the horizontal part of the duodenum were obtained and immediately frozen in liquid nitrogen. The rationale for taking duodenal samples was that this part of the intestine has a high turnover of 5-HT and recent reports suggesting an impairment of serotonergic function in the duodenum in IBS. 9

\section{ASSESSMENT OF KYNURENIC ACID AND 5-HT CONCENTRATIONS}

Blood samples were collected using pre-chilled K2EDTA tubes. To avoid oxidative breakdown, $1 \mathrm{ml}$ 1.4\% ascorbic acid (Sigma Aldrich, St. Louis, MO) was added to tubes. 
Platelet poor plasma samples were obtained by centrifuging tubes at $2000 \mathrm{~g} \mathrm{at} 4^{\circ} \mathrm{C}$ for 10 minutes. Supernatants were allocated and immediately frozen at $-80^{\circ} \mathrm{C}$ until analysis. Biopsy specimens were weighed and homogenized prior to analysis. Concentrations of 5HT and KYNA were determined by HPLC-MS as described previously. ${ }^{6}$

\section{STATISTICAL ANALYSES}

The descriptive statistical analyses were performed using SPSS 20.0 for windows (SPSS Inc., Chicago IL). Data were tested for normality by the Kolmogorov-Smirnoff test. Normally distributed data were analyzed by Student's test. Mann-Whitney U-test was used for non-parametric data. Coefficients for correlations were calculated according to Pearson and Spearmen, respectively. Data are expressed as mean \pm SEM, if not otherwise indicated. A Bonferroni correction was applied for multiple testing of correlations with psychiatric scores.

\subsection{RESULTS}

Table 1 describes demographic characteristics and psychological score. Distribution of IBS subtypes was as follows: $46 \%$ diarrhea predominant, $33 \%$ constipation predominant, $20 \%$ mixed subtype.

\section{Demographic characteristics and psychological score}

\begin{tabular}{l|lll}
\multicolumn{1}{c}{$\begin{array}{l}\text { IBS } \\
\text { Patients } \\
(\mathrm{N}=15)\end{array}$} & $\begin{array}{l}\text { Healthy } \\
\text { Controls } \\
(\mathrm{N}=15)\end{array}$ & p value \\
\hline Age & $44 \pm 13$ & $33 \pm 17$ & 0.06 \\
Gender & $46 \%$ male & $33 \%$ male & 0.35 \\
BMI & $27 \pm 5$ & $24 \pm 4$ & 0.08 \\
HADS & $3(0-10)$ & $0(0-4)$ & 0.001 \\
HADS-D & $2(0-6)$ & $0(0-4)$ & 0.007 \\
HADS-A & $0(0-4)$ & $0(0-0)$ & 0.03 \\
Global Severity Index (SCL-90) & $117(91-164)$ & $94(90-106)$ & $<0.001$
\end{tabular}




\section{Chapter 4}

Table 1: IBS = irritable bowel syndrome; BMI = body mass index; HADS = hospital anxiety and depression scale; SCL-90 = 90-item symptom checklist

\section{PLASMA AND MUCOSAL KYNA CONCENTRATIONS}

Plasma concentration of KYNA was significantly higher in IBS compared to healthy controls (2.96 \pm 0.33 vs $0.36 \pm 0.019 \mathrm{nmol} /, \mathrm{p}<0.0001$, see Figure $1 \mathrm{~A})$. On the contrary, mucosal concentrations of KYNA were significantly lower in IBS patients compared to healthy controls $(0.30 \pm 0.10$ vs $1.36 \pm 0.52 \mathrm{pmol} / \mathrm{mg}$ wet tissue, $p=0.02$, see Figure $B)$. $A$ significant negative correlation was found between mucosal and plasma concentration of KYNA ( $r=-0.45, p=0.03$ ), suggesting an inverse relationship between these two pools of KYNA. No association was found between age, gender or IBS subtype and KYNA concentrations.

\section{PLASMA AND MUCOSAL CONCENTRATIONS OF 5-HT}

A similar pattern of alterations was found in IBS with regards to 5-HT concentrations. Plasma concentration of 5-HT was significantly higher in IBS compared to healthy controls (26.2 \pm 4.7 vs $1.9 \pm 0.35 \mathrm{nmol} /, \mathrm{p}<0.0001$, see Figure C). In contrast, mucosal concentrations of 5-HT were significantly lower in IBS patients compared to healthy controls (12.6 \pm 3.4 vs $51.9 \pm 17.6 \mathrm{pmol} / \mathrm{mg}$ wet tissue, $p=0.008$, see Figure $D$ ). On the contrary, A significant negative correlation was found between mucosal and plasma concentration of $5-\mathrm{HT}(r=-0.42, p<0.05)$. No association was found between age, gender or IBS subtype and 5-HT concentrations.

A

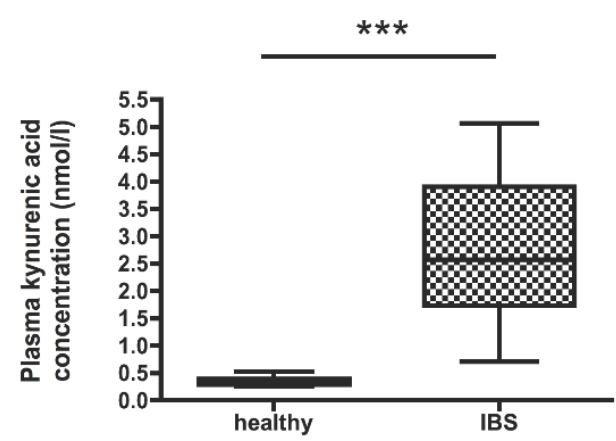

B

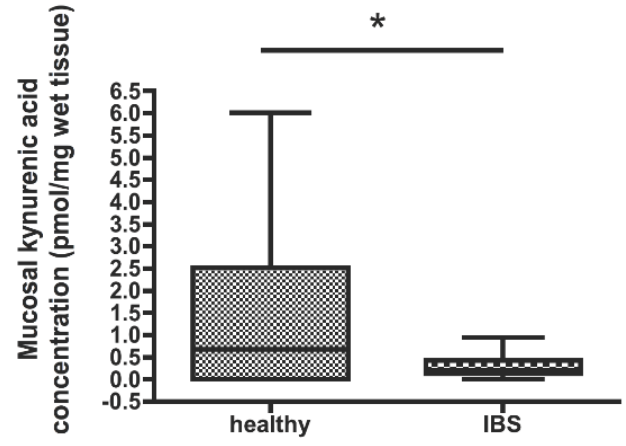



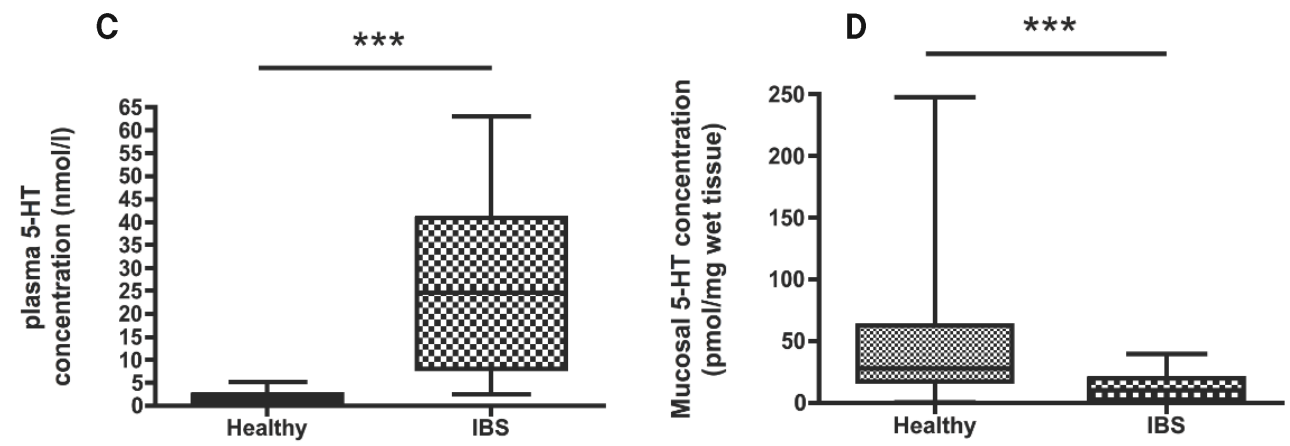

Figure 1: Plasma and mucosal concentrations of kynurenic acid and serotonin (5-HT) in patients with irritable bowel syndrome (IBS) and healthy controls, ${ }^{*} p<0.05$; ${ }^{* *} p<0.001$.

\section{CORRELATION BETWEEN 5-HT AND KYNA CONCENTRATIONS}

We also investigated whether there was any relation between levels of $5-\mathrm{HT}$ and KYNA. We found a significant negative correlation between plasma 5-HT and KYNA concentrations in both healthy controls $(r=-0.60, p=0.03)$ and in IBS patients $(r=-0.62$, $p=0.02$ ), suggesting an inverse relation. On the other hand, no correlation was found between mucosal concentrations of 5-HT and KYNA in either group (data not shown).

\section{CORRELATIONS WITH PSYCHOLOGICAL SCORE}

In IBS patients, a significant correlation was found between mucosal 5-HT and HADS score $(r=0.66, p=0.02)$ but not with the SCL-90 score $(r=0.15, p=0.64)$. When subdividing the HADS score into domains of depression and anxiety, mucosal 5-HT concentrations significantly correlated with the HADS-D score $(r=0.74, p=0.006)$ but not with HADS-A score $(r=0.25, p=0.42)$. Furthermore, a significant negative correlation was found between mucosal KYNA concentration and SCL-90 ( $r=-0.57$, p<0.05) but no correlation was found with the HADS score $(r=-0.34, p=0.27)$. In contrast to mucosal 5-HT and KYNA, no correlations were found for plasma 5-HT and KYNA concentrations and HADS or SCL 90 in IBS. It is important to note that following Bonferroni correction for multiple testing, only correlation between mucosal 5-HT and HADS-D score remained significant. 


\section{Chapter 4}

In healthy controls, no correlation was found between HADS scores and SCL-90 and either plasma or mucosal 5-HT concentrations or plasma or mucosal KYNA concentrations (data not shown).

\subsection{DISCUSSION}

A number of studies have found evidence for a dysregulation in the kynurenine pathway in IBS. The present study, although limited in sample size, is the first to report on both mucosal and systemic levels of KYNA. We found decreased mucosal levels and increased systemic levels of KYNA in IBS patients. KYNA is an antagonist at the N-methyl-Daspartate and nicotinic cholinoreceptors and an agonist at the orphan G-protein-coupled receptor GPR35, at which KYNA is one of the most potent endogenous agonists yet identified. ${ }^{10}$ GPR35 has been shown to have anti-nociceptive properties and has therefore been suggested as an important target to counteract inflammatory pain. ${ }^{11}$ The highest levels of GPR35 are in the intestine, and may hence play an important role in mediating the actions of KYNA. ${ }^{12}$ In the intestine, KYNA is known to have neuroprotective, anti-oxidative and anti-inflammatory properties and is believed to play a role in gut motility and sensory functions. ${ }^{13}$ Decreased mucosal concentrations of KYNA may therefore potentially contribute to functional neural, metabolic or inflammatory derangements that facilitate the development of gastrointestinal disorders, such as IBS.

Interestingly, we found increased plasma levels of KYNA in IBS, although it is important to note that findings in literature not consistent. ${ }^{14-16}$ Earlier studies showing increased plasma levels of kynurenic acid in patients with inflammatory bowel disease suggested that this increase may represent either a compensatory response to elevated activation of enteric neurons or a primary abnormality which induces a compensatory increase in gut activity. ${ }^{17}$ Systemic levels of kynurenine metabolites are largely under control of the liver by clearing any excess tryptophan through tryptophan dioxygenase (TDO), the initiating enzyme of the kynurenine pathway. Another source of kynurenine metabolites in plasma is derived from the activity of extrahepatic indoleamine-2,3-dioxygenase, largely expressed by the intestine. ${ }^{4}$ KYNA is synthesized from kynurenine by action of 
kynurenine aminotransferase, expressed primarily in the liver, intestine and kidney. ${ }^{18}$ The source of increased plasma KYNA concentrations is therefore most probably peripheral, as KYNA only poorly penetrates the blood-brain barrier. ${ }^{19}$ Increased systemic concentrations of KYNA may reflect either 1) increased activity of hepatic TDO or 2) increased release of KYNA from extrahepatic sources, such as the intestine. The former seems less likely since previous reports have suggested decreased TDO activity in IBS. ${ }^{15}$ The latter, however, is supported by the inverse relationship between intestinal mucosal and systemic levels of KYNA observed in our study. An increased release of KYNA from the mucosa into to systemic compartment could therefore result in mucosal KYNA deficiency, potentially contributing to intestinal dysfunction.

We also assessed 5-HT concentrations to ascertain their relation to KYNA concentrations. Levels of 5-HT in both plasma and mucosa showed a pattern similar to KYNA levels in IBS: significantly elevated plasma concentrations and decreased mucosal concentrations compared to healthy controls. Previous studies in IBS have also demonstrated higher plasma $5-\mathrm{HT}$ levels ${ }^{20}$ and lower $5-\mathrm{HT}$ levels in mucosa of IBS patients, ${ }^{21}$ although it is noteworthy that considerable discrepancies still exist in data from various reports. ${ }^{22}$

Plasma concentrations of 5-HT and KYNA were found to be inversely related in our study. However, the observation that plasma KYNA and 5-HT are both increased in IBS does not support the hypothesis that increased activation along the kynurenic pathway results in relative 5-HT deficiency contributing to disease development. Furthermore, no correlation was found in mucosal concentrations of $5-\mathrm{HT}$ and KYNA. It is important to note, that we measured only a single metabolite of the kynurenine pathway, which is not necessarily representative for the entire spectrum of kynurenine metabolites. Thus, it cannot be excluded that both alterations in 5-HT and kynurenine metabolism play a role in IBS, regardless of whether these changes are interdependent. A more complete profiling of mucosal and systemic concentration of these metabolites should therefore add to our understanding of the role of the kynurenine pathway in IBS. 


\section{Chapter 4}

We also ascertained whether these changes in 5-HT and KYNA concentration were associated with psychological state. We found no correlation with plasma concentration of 5-HT or KYNA and HADS or SCL-90 in IBS, which is in line with findings of Park et al. ${ }^{23}$ However, more interestingly, we observed a significant correlation between mucosal concentrations of 5-HT and psychological state in IBS patients. Apart from the disturbances in the 5-HT pathway, kynurenic pathway dysregulation has previously been associated with affective disorders. ${ }^{24}$ Plasma levels of kynurenic acid have either been shown to be decreased ${ }^{25}$ or unchanged in this patient group. ${ }^{26}$ Patients with depression (without gastrointestinal comorbidity) generally also have lowered plasma levels of 5HT. 24, 27 Considering the increased plasma levels of 5-HT and KYNA in IBS and the lack of correlation with psychological scores, we postulate that the changes in psychological state observed in IBS patients in our study may be secondary to alterations in gastrointestinal function, and in particular kynurenine and/or 5-HT metabolism. This is an important assumption since IBS patients have been shown to be hypervigilant regarding gastrointestinal symptoms. ${ }^{28}$ Potential alterations in gastrointestinal homeostasis may therefore be augmented by hypervigilance and contribute to increased disease burden. Consistent with this hypothesis is that cognitive behavioral therapy only indirectly improves bowel symptoms through improvement of mood and anxiety. ${ }^{29}$

Due to the preliminary nature of our findings and the relatively small sample size, further research is warranted to confirm the formulated hypotheses. It is also important to note that no differentiation was made with regards to different IBS subtypes. Furthermore, correlations with psychological scale should be interpreted with caution, as the small sample size and stringent multiple hypothesis corrections are factors possibly causing false negatives results. Findings of this study will therefore need to be substantiated in a larger group of patients. 


\subsection{REFERENCES}

1. Drossman DA, Camilleri M, Mayer EA, Whitehead WE. AGA technical review on irritable bowel syndrome. Gastroenterology 2002;123:2108-31.

2. Kim DY, Camilleri M. Serotonin: a mediator of the brain-gut connection. Am J Gastroenterol 2000;95:2698-709.

3. Berger M, Gray JA, Roth BL. The expanded biology of serotonin. Annu Rev Med 2009;60:355-66.

4. Keszthelyi D, Troost FJ, Masclee AA. Understanding the role of tryptophan and serotonin metabolism in gastrointestinal function. Neurogastroenterol Motil 2009;21:1239-49.

5. Stone TW. Kynurenines in the CNS: from endogenous obscurity to therapeutic importance. Prog Neurobiol 200 1;64:185-218.

6. Keszthelyi D, Troost FJ, Jonkers DM, van Donkelaar EL, Dekker J, Buurman WA, Masclee AA. Does acute tryptophan depletion affect peripheral serotonin metabolism in the intestine? Am J Clin Nutr 2012;95:603-8.

7. Miura H, Ozaki N, Sawada M, Isobe K, Ohta T, Nagatsu T. A link between stress and depression: shifts in the balance between the kynurenine and serotonin pathways of tryptophan metabolism and the etiology and pathophysiology of depression. Stress 2008;11:198-209.

8. Kaszaki J, Palasthy Z, Erczes D, Racz A, Torday C, Varga G, Vecsei L, Boros M. Kynurenic acid inhibits intestinal hypermotility and xanthine oxidase activity during experimental colon obstruction in dogs. Neurogastroenterol Motil 2008;20:53-62.

9. Foley S, Garsed K, Singh G, Duroudier NP, Swan C, Hall IP, Zaitoun A, Bennett A, Marsden C, Holmes G, Walls A, Spiller RC. Impaired uptake of serotonin by platelets from patients with irritable bowel syndrome correlates with duodenal immune activation. Gastroenterology 2011;140:1434-43 e 1.

10. Stone TW, Stoy N, Darlington LG. An expanding range of targets for kynurenine metabolites of tryptophan. Trends Pharmacol Sci 2012.

11. Cosi C, Mannaioni G, Cozzi A, Carla V, Sili M, Cavone L, Maratea D, Moroni F. Gprotein coupled receptor 35 (GPR35) activation and inflammatory pain: Studies on the antinociceptive effects of kynurenic acid and zaprinast. Neuropharmacology 2011;60:1227-31.

12. Wang J, Simonavicius N, Wu X, Swaminath G, Reagan J, Tian H, Ling L. Kynurenic acid as a ligand for orphan $G$ protein-coupled receptor GPR35. J Biol Chem 2006;281:22021-8.

13. Kaszaki J, Erces D, Varga G, Szabo A, Vecsei L, Boros M. Kynurenines and intestinal neurotransmission: the role of $\mathrm{N}$-methyl-D: -aspartate receptors. J Neural Transm 2012;119:211-23.

14. Wollny T RG, Pawlak D, Turecka-Kulesza E, Buczko W, Łaszewicz W. Kynurenic pathway metabolites in serum of patients with irritable bowel syndrome - possible role in the mechanism of visceral pain. Gastroenterologia Polska 2006;13:159-162.

15. Christmas DM, Badawy AA, Hince D, Davies SJ, Probert C, Creed T, Smithson J, Afzal M, Nutt DJ, Potokar JP. Increased serum free tryptophan in patients with diarrheapredominant irritable bowel syndrome. Nutr Res 2010;30:678-88. 
16. Fitzgerald P, Cassidy Eugene M, Clarke G, Scully P, Barry S, Quigley Eamonn MM, Shanahan F, Cryan J, Dinan Timothy G. Tryptophan catabolism in females with irritable bowel syndrome: relationship to interferon-gamma, severity of symptoms and psychiatric co-morbidity. Neurogastroenterol Motil 2008;20:1291-7.

17. Forrest CM, Youd P, Kennedy A, Gould SR, Darlington LG, Stone TW. Purine, kynurenine, neopterin and lipid peroxidation levels in inflammatory bowel disease. $J$ Biomed Sci 2002;9:436-42.

18. Walczak K, Dabrowski W, Langner E, Zgrajka W, Pilat J, Kocki T, Rzeski W, Turski WA. Kynurenic acid synthesis and kynurenine aminotransferases expression in colon derived normal and cancer cells. Scand J Gastroenterol 2011;46:903-12.

19. Fukui S, Schwarcz R, Rapoport SI, Takada Y, Smith QR. Blood-brain barrier transport of kynurenines: implications for brain synthesis and metabolism. J Neurochem 1991;56:2007-17.

20. Atkinson W, Lockhart S, Whorwell PJ, Keevil B, Houghton LA. Altered 5hydroxytryptamine signaling in patients with constipation- and diarrheapredominant irritable bowel syndrome. Gastroenterology 2006;130:34-43.

21. Coates MD, Mahoney CR, Linden DR, Sampson JE, Chen J, Blaszyk H, Crowell MD, Sharkey KA, Gershon MD, Mawe GM, Moses PL. Molecular defects in mucosal serotonin content and decreased serotonin reuptake transporter in ulcerative colitis and irritable bowel syndrome. Gastroenterology 2004;126:1657-64.

22. Mawe GM, Coates MD, Moses PL. Review article: intestinal serotonin signalling in irritable bowel syndrome. Aliment Pharmacol Ther 2006;23:1067-76.

23. Park SY, Park MH, Yoon KW, Cho SB, Lee WS, Park CH, Kim HS, Choi SK, Rew JS. Plasma 5-hydroxytryptamine concentration and its correlation with psychopathology in patients with irritable bowel syndrome. Gut Liver 2009;3:26-30.

24. Sarrias MJ, Artigas F, Martinez E, Gelpi E, Alvarez E, Udina C, Casas M. Decreased plasma serotonin in melancholic patients: a study with clomipramine. Biol Psychiatry 1987;22:1429-38.

25. Myint AM, Kim YK, Verkerk R, Scharpe S, Steinbusch H, Leonard B. Kynurenine pathway in major depression: evidence of impaired neuroprotection. J Affect Disord 2007;98:143-51.

26. Hughes MM, Carballedo A, McLoughlin DM, Amico F, Harkin A, Frodl T, Connor TJ. Tryptophan depletion in depressed patients occurs independent of kynurenine pathway activation. Brain Behav Immun 2012.

27. Gao HQ, Zhu HY, Zhang YQ, Wang LX. Reduction of cerebrospinal fluid and plasma serotonin in patients with post-stroke depression: A preliminary report. Clin Invest Med 2008;31:E351-6.

28. Posserud I, Svedlund J, Wallin J, Simren M. Hypervigilance in irritable bowel syndrome compared with organic gastrointestinal disease. J Psychosom Res 2009;66:399-405.

29. Jones M, Koloski N, Boyce P, Talley NJ. Pathways connecting cognitive behavioral therapy and change in bowel symptoms of IBS. J Psychosom Res 2011;70:278-85. 
Kynurenic acid in IBS 
Revisiting concepts of visceral nociception in irritable bowel syndrome

D. Keszthelyi, FJ. Troost, M. Simrén, S. Ludidi, JW. Kruimel, JM. Conchillo, AA. Masclee

European Journal of Pain 2012 Nov; 16(10):1444-54

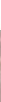




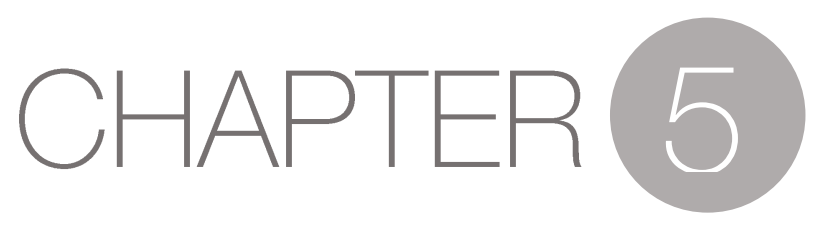

Mechanisms of visceral hypersensitivity 


\section{ABSTRACT}

Background and objective: Irritable bowel syndrome (IBS) is a common disorder characterized by abdominal pain related to defecation with a change in bowel habit. Patients with IBS often exhibit increased visceral sensitivity, which can be tested clinically by rectal balloon distension procedures. This paper aims to give an overview of mechanisms involved in visceral hypersensitivity in IBS by reviewing recent literature.

Databases and data treatment: A literature search in the electronic databases Pubmed and MEDLINE was executed using the search terms 'visceral pain' or 'visceral nociception' or 'visceral hypersensitivity' and 'irritable bowel syndrome.' Both original articles and review articles were considered for data extraction.

Results: Recent advances in molecular neurophysiology provide knowledge to better understand the underlying mechanism in pain generation in the human gut, in particular, in IBS patients. Sensitization of peripheral nociceptive afferents, more specifically high threshold afferents, has been proposed as one of the principle mechanism in the development of visceral hypersensitivity. On the other hand, central mechanisms also play an important role. In terms of clinical testing of visceral perception, considerable discrepancies remain, however, accross different centers.

Conclusion: Alterations in the modulatory balance of pro- and anti-nociceptive cental processing of noxious peripheral input may serve as in integrative hypothesis for explaining visceral hypersensitivity in IBS. Nevertheless, it remains troublesome to estimate the contribution of central and peripheral factors in visceral hypersensitivity, posing a challenge in determining effective therapeutic entities. 


\subsection{INTRODUCTION}

Irritable bowel syndrome (IBS) is a commonly seen disorder in clinical practice, affecting $10-15 \%$ of the population in developed countries. Referred to as a functional gastrointestinal disorder, it is often characterized by lower abdominal pain with disturbed defecation in absence of structural or biochemical abnormalities that might explain the symptoms. ${ }^{1}$ Despite being very common, the pathophysiology of IBS is incompletely understood, which poses problems in the search for effective therapeutic alternatives. Nonetheless, it is now widely accepted that an altered visceral sensitivity through abnormal endogenous pain processing plays an important role in the pathogenesis of IBS, ${ }^{2}$ although not all IBS patients exhibit visceral hypersensitivity when clinically tested using rectal distension. Both central and peripheral mechanisms have been suggested to be involved in the development of pain symptoms. Several studies have provided evidence that IBS is associated with a dysregulation of the brain-gut axis, with peripheral alterations dominating in some patients and disturbed central processing dominating in others. ${ }^{3}$ Recently, molecular findings have prompted interest in peripheral sensory physiology to explain pain and other symptoms in patients with IBS. Further knowledge on the exact mechanisms of pain generation will provide foundation for the development of potential therapeutic agents.

\subsection{NEUROANATOMY OF VISCERAL PAIN: NOCICEPTIVE AFFERENTS}

The biological function of pain sensation (nociception) is to detect potentially harmful stimuli. In the healthy situation, pain is produced only by noxious stimuli that are potentially or actually causing damage to tissue. When pain perception becomes too intense, it causes pain sensation without benefit and can therefore lead to considerable morbidity. This is generally true for somatic nociception. Earlier consensus considered visceral pain to merely being a variant of somatic pain. However, tissue injury (or threat of such injury) may not be required or necessary for induction of visceral pain, as it is for pain from somatic (mainly cutaneous) structures. The gastrointestinal tract is under normal conditions not a source of conscious sensory experiences apart from registration 
of physiological sensations such as fullness, satiety, hunger and urgency. While cutting, crushing or burning often fail to be detected, distension of hollow organs reliably causes sensations in humans, including pain. ${ }^{4}$ Unpleasant sensations are generally felt acutely or perceived as painful when stimuli exceed the physiological range or when sensory afferent are sensitized as a consequence of tissue injury or inflammation. ${ }^{5}$

Over the past few decades, researchers have attempted to identify the functional mechanisms and anatomical structures responsible for visceral pain generation. Several distinct anatomic pathways have been described morphologically and functionally in several species (rat, guinea pig, cat), but the most comprehensive studies have been executed on the murine intestinal wall.6 Accordingly, the innervation of the distal part of the colon and rectum is under control of two, anatomically and functionally distinct systems: the lumbar splanchnic nerves, which terminate in the thoracolumbar spinal cord, and the sacral pelvic nerves, which terminate in the lumbosacral spinal cord. Splanchnic and pelvic afferents follow the path of sympathetic and parasympathetic efferents that project to the gut wall and terminate within the muscle, mucosal epithelia and ganglia of the enteric nervous system.

Both systems contain different sensory fibers that can further be divided in the following categories: $^{7}$

> Mucosal afferents respond exclusively to fine tactile and chemical stimuli on the luminal surface. In the anal region, they are probably responsible for discrimination of solid, liquid and gas and for providing feedback from physiological stimuli such as the normal passage of fecal material through the gastrointestinal tract. ${ }^{8}$

> Muscular afferents respond to distension at physiological levels $(<20 \mathrm{mmHg})$ and provide input for the perception of distension or contraction. ${ }^{6}$ These afferents are considered to contribute to sustained filling, bloating or distension sensations.

> Serosal and mesenteric afferents respond to noxious levels of distension (>30 $\mathrm{mmHg}){ }^{9}$ They detect overdistension and high amplitude contractions.

> Muscular-mucosal afferents respond both to tactile and distension stimuli (both 
noxious and physiologicall. ${ }^{6}$ Their functional role is undetermined, but it is possible they may play a specialized role in detecting bolus or stool passage.

Regarding the functional characteristics of these afferents, consensus appears to be that there are fibers with low- and high thresholds to distension, fibers that are distension insensitive (probably mucosal afferents) and a separate class of mechanically insensitive fibers that respond only to chemical stimuli. Low-threshold mechanosensory afferents (sometimes referred to as "wide dynamic range" afferents) respond initially to physiological (innocuous) distension but continue to encode proportionally to organ distension into the noxious range. It is important to note that no matter how prolonged the innocuous stimulus may be, low-threshold afferents will not signal noxious events until the stimulus intensity increases above the noxious level. As a group, these afferents give greater magnitude responses throughout the noxious range than high-threshold fibres. ${ }^{10}$ High-threshold (or phasic) afferents, on the other hand, exert low resting activity and respond only to noxious intensities of organ distension. ${ }^{11}$ Although high-threshold mechanonociceptors are located in the serosal and mesenteric membranes, they have transduction sites in the submucosa which provides an explanation that inflammatory conditions in the intestinal wall can also sensitize these afferents. Moreover, inflammation seems to sensitize low-threshold afferents to lesser extent in comparison to highthreshold afferents and high-threshold serosal and mesenteric afferents appear to be responsible for post-inflammatory mechanical hypersensitivity. ${ }^{12}$

A number of visceral afferents that are silent at rest and are insensitive to all but highest intensity of mechanical stimulation have been suggested to be 'silent' nociceptors. ${ }^{11}$ They are assumed but not proven to play a role in the viscera similar to that observed in somatic pain. ${ }^{5}$ These afferents have been suggested to generate spontaneous activity and hence mechanosensitivity in the noxious range during or after inflammation. ${ }^{11}$ They may in fact constitute a different class of nociceptors for which colonic distension is not an adequate stimulus but become sensitized following inflammation, ischemia or in the presence of specific chemicals. However, recent studies by Hughes et al. found no 
evidence of recruitment of these 'silent afferents' following inflammation in mice. Therefore, post-inflammatory hypersensitivity is probably explained based on the existing population of afferents. ${ }^{12}$ More recently, on the other hand, Feng and Gebhart documented a significant proportion of these silent nociceptors in colorectal pelvic and lumbar splanchnic pathways in mice and demonstrated their ability to acquire mechanosensitivity under brief inflammatory conditions. However, this sensitization was short-lasting and reversible, not necessarily replicating pathophysiological circumstances. ${ }^{13}$ Therefore, the exact biological function of these nociceptors remains to be established.

It is still not known to which extent this current understanding of visceral nociceptors obtained from animal models can be extrapolated to the human situation. Very recently, recordings from human intestinal afferents were obtained from isolated intestine. Techniques have been developed to dissect mesenteric nerve bundles to enable direct electrophysiological recordings from human visceral afferents. ${ }^{14,15}$ The ability to perform such human recordings will potentially open new horizons in understanding human visceral signaling. In fact, the study by Jiang et al. presented preliminary evidence that the sub-populations of afferent as described in murine models exist in human tissue as well. ${ }^{15}$ Furthermore, Peiris et al. demonstrated that stimulation of human visceral afferents with an inflammatory 'soup,' containing prostaglandin, histamin, serotonin, bradykinin and ATP, resulted in an increased activation of spontaneously active units and recruitment of previously silent units. ${ }^{14}$ This adds additional evidence to the presumed role of 'silent' nociceptors in visceral nociception.

\subsection{MOLECULAR CHARACTERISTICS OF NOCICEPTORS}

The functional characteristics of nociceptive afferents may even be much more relevant than their anatomical location. The identification of nociceptors therefore often relies on cellular characterization, such as biochemical markers, rather than functional or anatomical definitions. Several pain sensing molecules (nociceptive transducers) have been identified in the last decades. Ion channels in the transient receptor potential (TRP) 
channel family have a role, to varying degree, in gastrointestinal chemo-, thermo- and mechanonociception (TRPV1, TRPA1, TRPV4), which has extensively been reviewed recently. ${ }^{5} 8$ Peiris et al. ${ }^{14}$ and Jiang et al. ${ }^{15}$ both demonstrated sensitivity to capsaicin, a well-known TRPV 1 agonist, which indicates that human visceral afferents express TRPV 1 receptors suggesting a role for TRPV 1 in modulating mechanosensivity. TRPV 1 receptors have in fact been shown to be up-regulated in mucosal biopsy specimens of IBS patients. ${ }^{16}$ Besides TRPV1, the TRPA 1 receptor seems to emerge as one of the key molecules involved in visceral mechanonociception. Experimental evidence suggests a mechanosensory role for TRPA 1 in 4 afferent subtypes: mucosal afferents in the lower gut, mesenteric and serosal afferents in the colon, and mucosal responses of the muscular/mucosal afferents in the colon. ${ }^{17}$ TRPA 1 also contributes to the tactile function of mucosal afferents, suggesting a multimodal mechano- and chemosensing function. ${ }^{17}$ The TRPA 1-mediated mechanosensitivity is also enhanced in inflammatory conditions associated with visceral hyperalgesia. ${ }^{17,} 18$ Furthermore, involvement of serotonergic pathways has been proposed in TRPA 1-mediated mechanonociception: TRPA1 is expressed by enterochromaffin cells and releases serotonin upon luminal stimulation including distension. ${ }^{19}$ Serotonin can activate different receptor subtypes, in particular 5$\mathrm{HT}_{3}$, that have been shown to be involved in visceral sensitivity. ${ }^{20}$ We anticipate that in future research, more attention will be given to the characterization of these signaling molecules.

\subsection{HYPERALGESIA, ALLODYNIA: A PERIPHERAL PERSPECTIVE}

Hyperalgesia and allodynia, terms originally used in reference to somatic sensation, have been adapted to studies of visceral sensation. It is well established that a substantial group, albeit not all IBS patients experience more pain than healthy controls, in particular during rectal disension. ${ }^{21}$ This physiological phenomenon can either be regarded as hyperalgesia or allodynia (usually grouped together under the term visceral hypersensitivity). An increase in perception in response to normally painful stimuli is referred to as hyperalgesia, whereas an increased sensation to normally innocuous stimuli 
is termed allodynia. In somatic nociception, hyperalgesia may reflect an increase in the excitability of tissue nociceptors, as well as neurons in the central nervous system (CNS) involved in nociceptive processing. This increase in excitability is referred to as sensitization. Allodynia appears to be conveyed by low-threshold afferents signaling to an already sensitized CNS. ${ }^{22}$ Although these mechanisms have been first described for somatic pain processing, new insights in the neuroanatomy of visceral pain may implicate the existence of similar processing in viscera. In case of IBS, an initial event, such as an inflammatory reaction or altered serotonergic transmission, can induce a change in the neurobiology of pain perception, resulting in altered peripheral pain processing. These peripheral changes can hence lead to sensitization of both peripheral and central nervous elements. For instance, inflammatory mediators such as bradykinins, tachykinins, prostaglandin, serotonin, ATP and protons can sensitize the molecules involved in pain perception such as TRPV1 or TRPA1, which in turn will be activated by sub-threshold stimuli. ${ }^{23,} 24$ Such sensitization of visceral afferents seems also evident in the human gut as recently demonstrated by Peiris et al using a 'soup' of inflammatory mediators to increase the firing rate of appendicular afferents. ${ }^{14}$

Serosal mechanosensitive afferents are highly sensitive to endogenously released substances such as bradykinin, serotonin and substance P. ${ }^{25}$ Furthermore, even mild inflammatory events that do not necessarily cause acute sensitization can induce chronic hypersensitivity. ${ }^{26}$ Such a peripheral sensitization of primary afferent neuron terminals within the gut results in a decrease in the intensity and/or amplitude of the stimulus required to initiate their depolarization and in an increase in the number and/or amplitude of neuronal discharges in response to a given chemical or mechanical stimulus.

\section{SEROTONIN}

A number of mechanisms have been proposed to play a pivotal role in the pathogenesis of visceral hypersensitivity in IBS. Accumulating evidence suggest that alterations in serotonergic signaling contribute to altered peripheral pain processing in IBS, which is not surprising given the important role of serotonin (5-hydroxytryptamine, 5-HT) in 
orchestrating intestinal function. ${ }^{27}$ Altered $5-\mathrm{HT}$ levels as well as increased number of enterochromaffin cells, responsible for the majority of $5-\mathrm{HT}$ synthesis in the gut, have been found in mucosal samples of IBS patients. ${ }^{28,} 29$ Mucosal 5-HT release has in fact been show to correlate with the intensity of perceived abdominal pain in IBS, ${ }^{29}$ and postprandial symptom exacerbation in female patients with diarrhea-predominant IBS (IBS-D) has been shown to be associated with increased levels of plasma $5-\mathrm{HT} .{ }^{30} \mathrm{~A}$ recent study using human enteric neurons showed that supernatants of biopsies from IBS patients released $5-\mathrm{HT}$ causing neural activation, which could be blocked by the $5-\mathrm{HT}_{3}$ receptor antagonist cilansetron. ${ }^{31}$ Also, treatment with the $5-\mathrm{HT}_{3}$ receptor antagonist alosetron significantly increased perception thresholds to colonic distension in IBS patients with diarrhea, ${ }^{32}$ although safety concerns have been raised recently regarding the use of serotonergic agents in the treatment of IBS. ${ }^{33}$ More recently, an experimental drug aimed at the selective inhibition of the enzyme responsible for 5-HT synthesis in enterochromaffin cells, tryptophan hydroxylase-1, has been demonstrated to improve symptoms in nonconstipating IBS patients. ${ }^{34}$ It remains to be established whether this inhibition of 5-HT synthesis can also have beneficial effects on visceral hypersensitivity. Although there appears to be consenus that alterations in serotonergic conditions is an important factor in the pathophysiology of IBS, changes observed in different studies are not entirely congruent. ${ }^{35}$ Also, a clinical study with the serotonin reuptake inhibitor fluoxetine found no significant effect on rectal hypersensitivity and abdominal pain in a mixed group of diarrhea and constipation-predominant IBS patients. ${ }^{36}$ The heterogenous nature of IBS and the complexity of serotorgic signalling and metabolism can provide a possible explanation for this inconsistency over the different studies. Either way, alterations in serotonergic conditions are most probably not the sole reason for visceral hypersensitivity and the constellation of symptoms observed in patients with IBS.

\section{MUCOSAL INFLAMMATION}

Another important mechanism of visceral hypersensitivity in IBS is related to sustained low-grade inflammation in the intestinal mucosa. Studies in patients with post-infectious IBS (PI-IBS), a condition which offers best insight into peripheral mechanisms involved in 
IBS, have shown an increased number of T-lymphocytes in the lamina propria 3 months following the initial infection. ${ }^{28}$ Several other studies have also demonstrated morphologically similar mucosal immune activation in non-PI-IBS patients. ${ }^{37-39}$ This immune activation is consistent with the hypothesis of low-grade immune activation in IBS and may also be involved in symptom generation in IBS. ${ }^{40}$ However, only about $50 \%$ of IBS patients show a significant infiltration of immunocytes - they remain undetectable in the remaining 50\%, even though they have the same symptoms as those with mild mucosal inflammation. ${ }^{37}$ It is also unclear whether this inflammation is sub-clinical or pathogenic and whether it effectively contributes to an altered sensitivity. ${ }^{41} \mathrm{~A}$ prospective study by Gwee et al. showed that patients who developed IBS following an enteral infection had higher mucosal inflammatory cell counts compared to those who did not develop IBS, however, no differences were found in rectal sensitivity. ${ }^{42}$

Nevertheless, post-inflammatory hyperalgesia is a well-documented phenomenon. ${ }^{43,44} \mathrm{~A}$ possible explanation for an initial infection resulting in post-inflammatory hyperalgesia could be explained by an impairment of the intestinal barrier function. Epithelial cells of the mucosa are bound together by tight junctions and form a selective barrier which is continually challenged by luminal pathogens. This interaction allows a physiological level of immune activation, which is necessary to protect the gut wall from the hostile luminal environment. This basal level of inflammation does not affect the overall integrity of the epithelial barrier. In case of an intestinal infection, the acute inflammatory reaction may potentially impair epithelial barrier function, as has been demonstrated in patients with $\mathrm{PI}-\mathrm{IBS} .{ }^{45}$ This allows luminal content to enter the lamina propria thereby triggering further immune response. Exposure of sensory afferent endings to these inflammatory mediators and/or luminal content can lead to sustained sensitization of afferents contributing to visceral hyperalgesia even when the initial infection has resolved. This idea suggests that low-grade mild mucosal inflammation seen in IBS is rather an epiphenomenon of impaired barrier function. Several groups have in fact demonstrated alterations in barrier function $\mathrm{PI}-\mathrm{IBS}, 45,46$ but also non-PI-IBS, with these changes being mostly observed in IBS-D. ${ }^{47-50}$ Furthermore, a subset of IBS-D patients with increased intestinal permeability have also 
been shown to exhibit visceral hypersensitivity. ${ }^{51}$ We therefore postulate that impairment of the intestinal barrier, possibly through an intestinal infection, may very well be one of the key, albeit not exclusive, mechanisms in visceral hypersensitivity in IBS.

Intestinal infection, on the other hand, can also cause alterations in gut microbiota by a loss of containment of the indigenous colonic microbial community or small intestinal bacterial overgrowth, which can also contribute to visceral afferent sensitization in IBS, 52 possibly also through interference with the intestinal barrier function. ${ }^{53}$ Further studies are needed to establish the role of these peripheral processes and to which extent they can contribute to alterations in pain perception in IBS.

The argument that there is indeed a selective nociceptive pathway in the gastrointestinal tract that is prone to sensitization underlines the role for peripheral factors in the pathogenesis of visceral hypersensitivity in IBS. On the other hand, IBS patients often complain of bloating, cramps, urgency and discomfort in addtion to experiencing pain. This observation argues against a selective sensitization of a specialized peripheral nociceptive pathway. Indeed, in patients with functional dyspepsia, another functional gastrointestinal disorder characterized by altered visceral perception, increased perception of both painful and non-painful stimuli was observed, pointing to sensitization of both selective nociceptive and multimodal afferent pathways. ${ }^{54}$ It is noteworthy futhermore, that on average, only $50 \%$ of IBS patients demonstrate visceral hypersensitivity, although more than $90 \%$ of them complain of abdominal pain and cramping. ${ }^{55-57}$ Also, IBS patients experience pain for long periods of time, which cannot be related to ongoing luminal distention at noxious levels. Even more so, nociceptor activation alone may not be sufficient or even necessary to cause chronic pain or discomfort. ${ }^{58}$ This suggests that central mechanisms are also involved in the processing of visceral sensations. 


\subsection{THE ROLE FOR CENTRAL PAIN PROCESSING MECHANISMS IN VISCERAL HYPERSENSITIVITY}

Earlier reports have acknowledged that an increased peripheral input to the CNS can trigger neuroplastic changes, which result in central sensitization, amplifying further signals originating from the affected viscus. ${ }^{59}$ More recently, imaging studies have indeed demonstrated that increased peripheral sensory input can alter brain activity. ${ }^{60}$ Enlarged viscerosomatic referral observed in IBS also suggest the role for sensitization of spinal neurons that have viscerosomatic convergence and exhibit somatotopic overlap with the gut. 56

On the other hand, it has also been established that IBS patients appear to have disrupted emotional modulation of neural responses to visceral stimuli, possibly reflecting the neural basis for altered visceral interoception by stress and negative emotion. ${ }^{61}$ Furthermore, it has also been proposed that increased sensitivity to visceral stimuli in IBS is explained by hypervigilance and an increased tendency to report pain, rather than enhanced neurosensory input.62 These phenomena can be explained by cortical processes prominent in IBS, such as vigilance, distraction, expectation, and increased symptomdirected anxiety, which exert profound descending modulation via brainstem-spinal pathways. ${ }^{23}$ Therefore, it remains unclear whether abnormal brain activation observed in imaging studies are caused by hypersensitive nerves within the gut, by abnormal processing of afferent input at spinal level, or by abnormal cognitive-affective supraspinal processing. ${ }^{63}$ More recent reports highlight that an individual's pain reponse is largely dependent on a dynamic balance between pain inhibition and facilitation, orchestrated by different anatomic levels of pain processing: spinal, medullar, limbic and cortical. ${ }^{64}$

Considering our current scientific knowledge, we can generally assume that all levels of visceral pain processing (peripheral sensory input, spinal cord and supraspinal centra) contribute to the development of hypersensitivity in IBS, but the relative contribution of peripheral versus central factors may differ between subjects, possibly reflecting the heterogenous character of the disorder. Accordingly, peripheral and/or central 
sensitization alters the perception of peripheral sensory input from the gastrointestinal tract and summation of peripheral sensory input and central modulatory processing determines the overall sensory perception of an individual. An altered modulatory balance of inbitition and facilitation may therefore well be a uniting pathophysiological mechanism for visceral hypersensitivity in IBS (see Figure 1). Unraveling this complexity of visceronociception will potentially open new horizons in the development novel therapeutic entities in treating pain symptoms in IBS.

\section{VISCERAL HYPERSENSITIVITY}

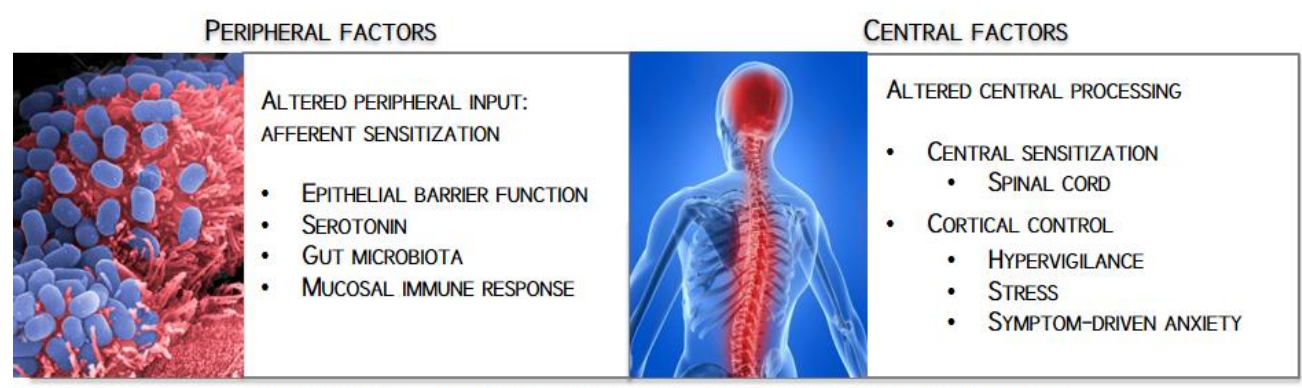

MODULATORY BALANCE OF NOCICEPTIVE INHIBITION AND FACILITATION

Figure 1: Mechanisms involved in visceral hypersensitivity.

\subsection{ASSESSMENT OF VISCERAL PAIN IN IBS: A PERIPHERAL APPROACH}

Since Ritchie et al. ${ }^{65}$ first demonstrated increased sensory responses to rectal balloon distension in IBS patients, numerous studies have suggested that this hypersensitivity could be a biological marker of IBS.2, 66-68 Rectal visceral sensitivity can be tested by using polyethylene bags in conjunction with a barostat. ${ }^{69}$ The barostat is a device that maintains a constant pressure within an air-filled polyethylene bag and can measure variations in rectal tone by recording changes in the intrarectal pressure and volume. Rectal distension can be done according to a certain protocol, during which different pressures are applied to the rectal wall. During this procedure, the intensity and quality of perception can be measured by means of rating scales, which may be analogue, numerical or descriptive. 
During rectal distension, several parameters of visceral sensitivity can be collected to assess overall perceptional status. These include perceived intensity of the stimulus, extent of the viscerosomatic referral area and the threshold at which different sensations (pain, discomfort, urge) appear during the distension protocol. ${ }^{56}$ These components of visceral perception are not uniformly used by different groups making comparison of study results troublesome. The majority of the groups investigating visceral perception using the barostat techniques have focused on identifying the thresholds for perception in IBS patients as these have been shown to be lowered in comparison to healthy volunteers.

Accordingly, assuming that healthy controls do not have sensitized peripheral afferents or CNS, rectal distension protocols can be used to assess the normal pain perception threshold, i.e. an attempt can be made to define the least intense stimulus that can consciously be perceived as pain. This could then be identified as the pain perception threshold. Thresholds can be defined for the following categories: first perception (rectal sensation threshold, the pressure level that is first perceived, not necessarily being painful), first pain (rectal pain threshold, the pressure level at which pain is first perceived) and pain tolerance threshold (the pressure level at which the measurement needs to be terminated due to increased pain perception, see Figure 2). Moreover, thresholds for other sensations, such as gas, urge to defecate and discomfort have been used in different distention protocols, as well. Several recent studies have found that there is no significant difference in the first perception threshold and the first pain threshold between IBS patients compared to healthy volunteers. 68,70 These are pressure levels at which only low-threshold mechanoreceptors respond. However, pain tolerance thresholds are significantly lower in IBS.68, 70 This implies that lowered sensory thresholds in IBS are mainly restricted to noxious stimuli, thus IBS patients could exhibit hyperalgesia, but not allodynia, an observations also supported by the study of Camilleri et al. ${ }^{71}$ Furthermore, a study investigating the specificity and the sensitivity of the rectal barostat in IBS patients found that only severe hypersensitivity was specific for IBS.66 This could be explained by an increased sensitization of high-threshold but not low-threshold afferents, 


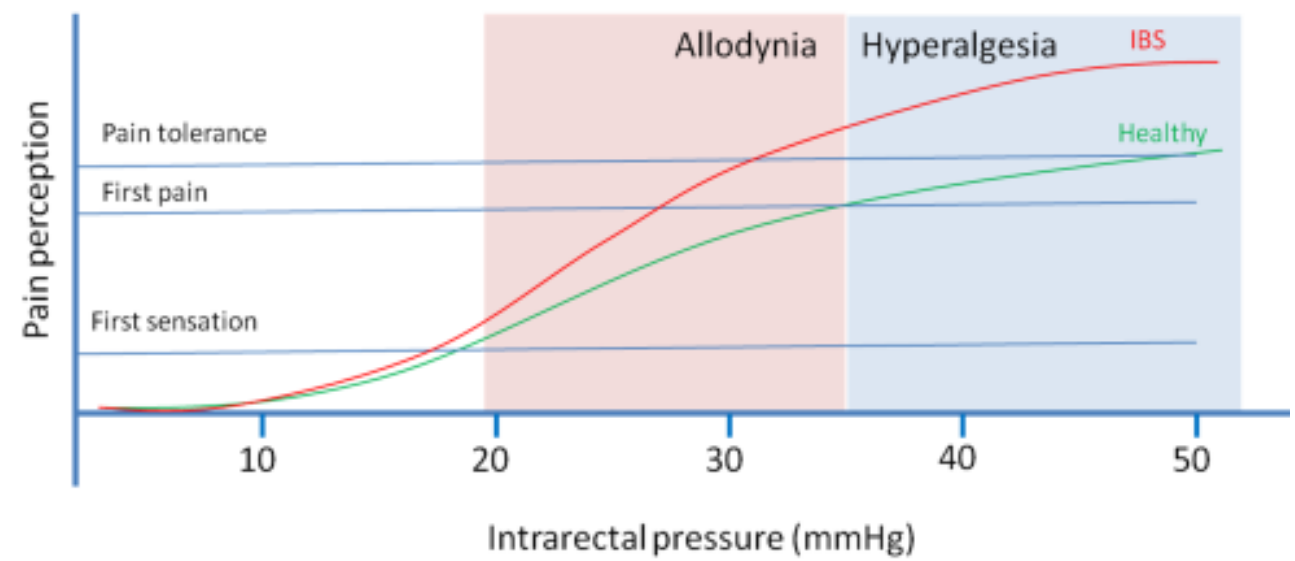

Figure 2: Schematic diagram of visceral perception induced by rectal distension in healthy volunteers and irritable bowel syndrome (IBS) patients.

as observed recently in a murine model. ${ }^{12}$ Although the high-threshold serosal and mesenteric afferents have shown not to reduce their minimum threshold to distension after inflammation, more of them are recruited above threshold and they generate a larger response, indicating visceral hyperalgesia. ${ }^{12}$ These afferents are particularly chemosensitive to inflammatory and enteroendocrine mediators such as serotonin, which suggests a role for nociception in conditions in which sensitization of visceral afferents is involved. Accepting that these afferents are at least in part responsible for the development of visceral hypersensitivity in IBS, rectal distensions theoretically offer an ideal tool to assess perceptional status in these patients.

Although increased perception to mechanical distension remains the best characterized clinical manifestation, certainly not all IBS patients exhibit this hypersensitivity. Perhaps this is due to the incomplete understanding of mechanisms underlying visceral pain perception during a barostat procedure. Techniques to quantify perceptual abnormalities related to visceral sensations, recently reviewed by Andresen, such as rectal distension by barostat methodology, remain suboptimal. ${ }^{72}$ Even though numerous groups have reported lowered rectal pain thresholds in IBS patients. $66,68,70$ these groups use different distension protocols. It has been demonstrated earlier that rapid phasic distensions 
preferentially stimulate serosal receptors and that more IBS patients seem hypersensitive when applying a protocol with rapid distensions as opposed to stepwise or ramp distensions. 57, 73, 74 Furthermore, complex distension protocols with non-random presentation of stimuli such as double random staircase distension reduce psychological response bias by making the stimulus unpredictable to the subject. ${ }^{75}$ IBS patients may be particularly prone to such bias due to hypervigilance and anticipatory anxiety, even though it is to be established if this anxiety is a cause or consequence of increased visceral perception. However, these complex protocols usually require more distension levels and increasing the number of interrogations about sensations may introduce bias or errors in a fatigued and stressed or disinterested participant.

Moreover, there are considerable differences in definitions used for pain thresholds among laboratories. Some groups define "threshold" as grade $3 / 5$ or 3/10 on the ordinal (verbal) or visual analogue scale for pain.66, 71 Others consider rectal sensation as a continuum ranging from defecatory urge, discomfort and eventually pain. ${ }^{56}$ Furthermore, there is no consensus with regards to the threshold defining the boundary between normal and exaggerated sensitivity, i.e. to discriminate between normo- and hypersensitivity. A study by Bouin et al. suggested a pain tolerance threshold of 40 $\mathrm{mmHg}$, which correctly excluded the diagnosis of IBS in $90 \%$ of the non-IBS cases. ${ }^{66}$ It is noteworthy that this group employed an ascending phasic distention, which maximizes hypervigilance. In other studies, the average pain threshold was $28 \mathrm{mmHg}$ in IBS patients, and $35 \mathrm{mmHg}$ in healthy controls, ${ }^{67,76}$ so one could argue that $40 \mathrm{mmHg}$ of pressure is already in the noxious range which might be less relevant as normal rectal pressures do not go above physiological levels. On the other hand, these higher pressure levels might be important in terms of distinguishing IBS patients from healthy volunteers. Given the understanding of visceral nociception obtained from clinical and experimental data, we can assume that high-threshold mechanoreceptors, which are prone to sensitize, can at least in part be accounted for the hypersensitive responses during rectal distension in IBS patients. Therefore, visceral perception protocols involving random phasic distensions should be optimized to stimulate these receptors for a more efficient assessment of 
perceptional status. Furthermore, as no widely accepted definition of visceral hypersensitivity is currently available, the use of a standard distension protocols and pain assessment methods in all centers would be desirable. We also suggest using all three categories/qualities (first sensation, first pain, pain tolerance) to be able to assess complete perception status. Moreover, investigation of the perceived intensity of the given stimulus as well as recording the viscerosomatic referral area can give extra information on processing of visceral stimuli.2, 56 On this basis, clear-cut definitions for normo- and hypersensitivity can be defined which allows better characterization of the sensitivity status in patients with IBS.

\subsection{FUTURE DIRECTIONS}

Novel imaging studies have provided further insight into mechanisms involved in pathological nociceptive signaling in IBS. Alterations in the modulatory balance of proand anti-nociceptive cental processing of peripheral input have received increasing attention in serving as an integrative mechanism for visceral hypersensitivity. This hypothesis could also provide an explanation for the fact that not all IBS patients exhibit this hypersensitivity: intact central (spinal and/or cortical) nociceptive control has the capacity to suppress noxious peripheral input from afferents, regardless whether these are sensitized, hereby ensuring that these sensations remain below a conscious level. Accumulating evidence from brain imaging studies confirms the role for corticol mechanisms in visceral nociception, however, these techniques fail to provide information on alterations in peripheral nociception. Therefore, it remains troublesome to estimate the contribution of central and peripheral factors in visceral hypersensitivity. Integrative approaches, in which both central and peripheral nociception is examined, could possibly provide explanations for this unanswered question.

\subsection{CONCLUSION}

The last decade has seen a surge of interest in the processing of augmented visceral sensitivity of the gut in several diseased states, especially IBS. For further advance of 


\section{Chapter 5}

this discipline, novel diagnostic tests and treatments are necessary but these await a more detailed understanding of the mechanisms and pathophysiology of visceral sensation. Ultimately, the relevance of the concept will depend on proof that symptoms are attributed to altered sensitivities and that therapies aimed at the correction of hypersensitivity result in clinical benefit. A prerequisite for such correction is a more thorough understanding of the pathways, mechanisms, structures, receptors, transmitters and other mediators involved in visceral sensitivity. With reports emerging about the varieties of mechanisms involved in the pathological conditions of visceral pain, we anticipate that better characterization of visceral nociception will be achieved relatively soon, which will open new horizons in the therapy of chronic visceral pain. As visceral hypersensitivity remains a paramount clinical phenomenon observed in IBS, the rectal disension using the barostat technique has been proven to be a valid human experimental model for visceral pain and is able to detect changes in algesia. Further insight in visceral nociception will also increase the value of rectal distension is assessing changes in visceral perception. 


\subsection{REFERENCES}

1. Drossman DA, Camilleri M, Mayer EA, Whitehead WE. AGA technical review on irritable bowel syndrome. Gastroenterology 2002;123:2 108-31.

2. Mertz H, Naliboff B, Munakata J, Niazi N, Mayer EA. Altered rectal perception is a biological marker of patients with irritable bowel syndrome. Gastroenterology 1995; 109:40-52.

3. Azpiroz F, Bouin M, Camilleri M, Mayer EA, Poitras P, Serra J, Spiller RC. Mechanisms of hypersensitivity in IBS and functional disorders. Neurogastroenterol Motil 2007; 19:62-88.

4. Cervero F. Sensory innervation of the viscera: peripheral basis of visceral pain. Physiol Rev 1994;74:95-138.

5. Knowles $\mathrm{CH}$, Aziz Q. Basic and clinical aspects of gastrointestinal pain. Pain 2009; 141:191-209.

6. Brierley SM, Jones RC, 3rd, Gebhart GF, Blackshaw LA. Splanchnic and pelvic mechanosensory afferents signal different qualities of colonic stimuli in mice. Gastroenterology 2004; 127:166-78.

7. Brierley SM. Molecular basis of mechanosensitivity. Auton Neurosci 2010;153:5868.

8. Blackshaw LA, Brierley SM, Hughes PA. TRP channels: new targets for visceral pain. Gut 2010;59:126-35.

9. Brierley SM, Page AJ, Hughes PA, Adam B, Liebregts T, Cooper NJ, Holtmann G, Liedtke W, Blackshaw LA. Selective role for TRPV4 ion channels in visceral sensory pathways. Gastroenterology 2008;134:2059-69.

10. Gebhart GF. Pathobiology of visceral pain: molecular mechanisms and therapeutic implications IV. Visceral afferent contributions to the pathobiology of visceral pain. Am J Physiol Gastrointest Liver Physiol 2000;278:G834-8.

11. Robinson DR, Gebhart GF. Inside information: the unique features of visceral sensation. Mol Interv 2008;8:242-53.

12. Hughes PA, Brierley SM, Martin CM, Brookes SJ, Linden DR, Blackshaw LA. Postinflammatory colonic afferent sensitisation: different subtypes, different pathways and different time courses. Gut 2009;58:1333-41.

13. Feng B, Gebhart GF. Characterization of silent afferents in the pelvic and splanchnic innervations of the mouse colorectum. Am J Physiol Gastrointest Liver Physiol 2011;300:G 170-80.

14. Peiris M, Bulmer DC, Baker MD, Boundouki G, Sinha S, Hobson A, Lee K, Aziz Q, Knowles $\mathrm{CH}$. Human visceral afferent recordings: preliminary report. Gut 2011;60:204-8.

15. Jiang W, Adam IJ, Kitsanta P, Tiernan J, Hill C, Shorthouse A, Grundy D. 'First-inman': characterising the mechanosensitivity of human colonic afferents. Gut 2011;60:281-2.

16. Akbar A, Yiangou Y, Facer P, Walters JR, Anand P, Ghosh S. Increased capsaicin receptor TRPV 1-expressing sensory fibres in irritable bowel syndrome and their correlation with abdominal pain. Gut 2008;57:923-9.

17. Brierley SM, Hughes PA, Page AJ, Kwan KY, Martin CM, O'Donnell TA, Cooper NJ, 
Harrington AM, Adam B, Liebregts T, Holtmann G, Corey DP, Rychkov GY, Blackshaw LA. The ion channel TRPA1 is required for normal mechanosensation and is modulated by algesic stimuli. Gastroenterology 2009;137:2084-2095 e3.

18. Cattaruzza F, Spreadbury I, Miranda-Morales M, Grady EF, Vanner S, Bunnett NW. Transient receptor potential ankyrin-1 has a major role in mediating visceral pain in mice. Am J Physiol Gastrointest Liver Physiol 2010;298:G81-91.

19. Nozawa K, Kawabata-Shoda E, Doihara H, Kojima R, Okada H, Mochizuki S, Sano Y, Inamura $\mathrm{K}$, Matsushime $\mathrm{H}$, Koizumi $\mathrm{T}$, Yokoyama $\mathrm{T}$, Ito $\mathrm{H}$. TRPA 1 regulates gastrointestinal motility through serotonin release from enterochromaffin cells. Proc Natl Acad Sci U S A 2009;106:3408-13.

20. Greenwood-van Meerveld B. Importance of 5-hydroxytryptamine receptors on intestinal afferents in the regulation of visceral sensitivity. Neurogastroenterol Motil 2007;19 Suppl 2:13-8.

21. van der Veek PP, Van Rood YR, Masclee AA. Symptom severity but not psychopathology predicts visceral hypersensitivity in irritable bowel syndrome. Clin Gastroenterol Hepatol 2008;6:321-8.

22. Koltzenburg M, Lundberg LE, Torebjork HE. Dynamic and static components of mechanical hyperalgesia in human hairy skin. Pain 1992;51:207-19.

23. Anand $P, A z i z Q$, Willert $R$, van Oudenhove L. Peripheral and central mechanisms of visceral sensitization in man. Neurogastroenterol Motil 2007;19:29-46.

24. Bueno L, Fioramonti J, Garcia-Villar R. Pathobiology of visceral pain: molecular mechanisms and therapeutic implications. III. Visceral afferent pathways: a source of new therapeutic targets for abdominal pain. Am J Physiol Gastrointest Liver Physiol 2000;278:G670-6.

25. Sengupta JN. Visceral pain: the neurophysiological mechanism. Handb Exp Pharmacol 2009:31-74.

26. Jones RC, 3rd, Otsuka E, Wagstrom E, Jensen CS, Price MP, Gebhart GF. Short-term sensitization of colon mechanoreceptors is associated with long-term hypersensitivity to colon distention in the mouse. Gastroenterology 2007;133:18494.

27. Spiller R. Recent advances in understanding the role of serotonin in gastrointestinal motility in functional bowel disorders: alterations in 5-HT signalling and metabolism in human disease. Neurogastroenterol Motil 2007;19 Suppl 2:25-31.

28. Dunlop SP, Jenkins D, Neal KR, Spiller RC. Relative importance of enterochromaffin cell hyperplasia, anxiety, and depression in postinfectious IBS. Gastroenterology 2003;125:1651-9.

29. Cremon C, Carini G, Wang B, Vasina V, Cogliandro RF, De Giorgio R, Stanghellini V, Grundy D, Tonini M, De Ponti F, Corinaldesi R, Barbara G. Intestinal serotonin release, sensory neuron activation, and abdominal pain in irritable bowel syndrome. Am J Gastroenterol 2011;106:1290-8.

30. Houghton LA, Atkinson W, Whitaker RP, Whorwell PJ, Rimmer MJ. Increased platelet depleted plasma 5-hydroxytryptamine concentration following meal ingestion in symptomatic female subjects with diarrhoea predominant irritable bowel syndrome. Gut 2003;52:663-70.

31. Buhner S, Li Q, Vignali S, Barbara G, De Giorgio R, Stanghellini V, Cremon C, Zeller 
F, Langer R, Daniel H, Michel K, Schemann M. Activation of human enteric neurons by supernatants of colonic biopsy specimens from patients with irritable bowel syndrome. Gastroenterology 2009;137:1425-34.

32. Delvaux M, Louvel D, Mamet JP, Campos-Oriola R, Frexinos J. Effect of alosetron on responses to colonic distension in patients with irritable bowel syndrome. Aliment Pharmacol Ther 1998; 12:849-55.

33. Ford AC, Brandt LJ, Young C, Chey WD, Foxx-Orenstein AE, Moayyedi P. Efficacy of 5-HT3 antagonists and 5-HT4 agonists in irritable bowel syndrome: systematic review and meta-analysis. Am J Gastroenterol 2009;104:1831-43; quiz 1844.

34. Brown PM, Drossman DA, Wood AJ, Cline GA, Frazier KS, Jackson Jl, Bronner J, Freiman J, Zambrowicz B, Sands A, Gershon MD. The tryptophan hydroxylase inhibitor LX1031 shows clinical benefit in patients with nonconstipating irritable bowel syndrome. Gastroenterology 2011;141:507-16.

35. Mawe GM, Coates MD, Moses PL. Review article: intestinal serotonin signalling in irritable bowel syndrome. Aliment Pharmacol Ther 2006;23:1067-76.

36. Kuiken SD, Tytgat GN, Boeckxstaens GE. The selective serotonin reuptake inhibitor fluoxetine does not change rectal sensitivity and symptoms in patients with irritable bowel syndrome: a double blind, randomized, placebo-controlled study. Clin Gastroenterol Hepatol 2003;1:219-28.

37. Cremon C, Gargano L, Morselli-Labate AM, Santini D, Cogliandro RF, De Giorgio R, Stanghellini V, Corinaldesi R, Barbara G. Mucosal immune activation in irritable bowel syndrome: gender-dependence and association with digestive symptoms. Am J Gastroenterol 2009; 104:392-400.

38. Ohman L, Isaksson S, Lindmark AC, Posserud I, Stotzer PO, Strid H, Sjovall H, Simren M. T-cell activation in patients with irritable bowel syndrome. Am J Gastroenterol 2009; 104:1205-12.

39. Ohman L, Lindmark AC, Isaksson S, Posserud I, Strid H, Sjovall H, Simren M. B-cell activation in patients with irritable bowel syndrome (IBS). Neurogastroenterol Motil 2009;21:644-50, e27.

40. Barbara G. Mucosal barrier defects in irritable bowel syndrome. Who left the door open? Am J Gastroenterol 2006;101:1295-8.

41. Sarna SK. Lessons Learnt from Post-Infectious IBS. Front Physiol 2011;2:49.

42. Gwee KA, Leong YL, Graham C, McKendrick MW, Collins SM, Walters SJ, Underwood $\mathrm{JE}$, Read NW. The role of psychological and biological factors in postinfective gut dysfunction. Gut 1999;44:400-6.

43. Hughes PA, Brierley SM, Blackshaw LA. Post-inflammatory modification of colonic afferent mechanosensitivity. Clin Exp Pharmacol Physiol 2009;36:1034-40.

44. Vergnolle N. Visceral afferents: what role in post-inflammatory pain? Auton Neurosci 2010;153:79-83.

45. Marshall JK, Thabane M, Garg AX, Clark W, Meddings J, Collins SM. Intestinal permeability in patients with irritable bowel syndrome after a waterborne outbreak of acute gastroenteritis in Walkerton, Ontario. Aliment Pharmacol Ther 2004;20:1317-22.

46. Spiller RC, Jenkins D, Thornley JP, Hebden JM, Wright T, Skinner M, Neal KR. Increased rectal mucosal enteroendocrine cells, T lymphocytes, and increased gut 
permeability following acute Campylobacter enteritis and in post-dysenteric irritable bowel syndrome. Gut 2000;47:804-11.

47. Dunlop SP, Hebden J, Campbell E, Naesdal J, Olbe L, Perkins AC, Spiller RC. Abnormal intestinal permeability in subgroups of diarrhea-predominant irritable bowel syndromes. Am J Gastroenterol 2006;101:1288-94.

48. Gecse K, Roka R, Ferrier L, Leveque M, Eutamene H, Cartier C, Ait-Belgnaoui A, Rosztoczy A, Izbeki F, Fioramonti J, Wittmann T, Bueno L. Increased faecal serine protease activity in diarrhoeic IBS patients: a colonic lumenal factor impairing colonic permeability and sensitivity. Gut 2008;57:591-9.

49. Piche T, Barbara G, Aubert P, Bruley des Varannes S, Dainese R, Nano JL, Cremon C, Stanghellini V, De Giorgio R, Galmiche JP, Neunlist M. Impaired intestinal barrier integrity in the colon of patients with irritable bowel syndrome: involvement of soluble mediators. Gut 2009;58:196-201.

50. Bertiaux-Vandaele N, Youmba SB, Belmonte L, Lecleire S, Antonietti M, Gourcerol G, Leroi AM, Dechelotte P, Menard JF, Ducrotte P, Coeffier M. The expression and the cellular distribution of the tight junction proteins are altered in irritable bowel syndrome patients with differences according to the disease subtype. Am $J$ Gastroenterol 2011;106:2165-73.

51. Zhou Q, Zhang B, Verne GN. Intestinal membrane permeability and hypersensitivity in the irritable bowel syndrome. Pain 2009;146:41-6.

52. Al-Khatib K, Lin HC. Immune activation and gut microbes in irritable bowel syndrome. Gut Liver 2009;3:14-9.

53. Rescigno M. The intestinal epithelial barrier in the control of homeostasis and immunity. Trends Immunol 2011;32:256-64.

54. Vandenberghe J, Vos R, Persoons P, Demyttenaere K, Janssens J, Tack J. Dyspeptic patients with visceral hypersensitivity: sensitisation of pain specific or multimodal pathways? Gut 2005;54:914-9.

55. Kuiken SD, Lindeboom R, Tytgat GN, Boeckxstaens GE. Relationship between symptoms and hypersensitivity to rectal distension in patients with irritable bowel syndrome. Aliment Pharmacol Ther 2005;22:157-64.

56. Posserud I, Syrous A, Lindstrom L, Tack J, Abrahamsson H, Simren M. Altered rectal perception in irritable bowel syndrome is associated with symptom severity. Gastroenterology 2007; 133:1113-23.

57. Lembo T, Munakata J, Mertz H, Niazi N, Kodner A, Nikas V, Mayer EA. Evidence for the hypersensitivity of lumbar splanchnic afferents in irritable bowel syndrome. Gastroenterology 1994;107:1686-96.

58. Levinthal DJ, Bielefeldt K. Differences in post-inflammatory hypersensitivity between splanchnic and pelvic afferents: mechanisms and implications for human disease. Gut 2009;58:1317-8.

59. Giamberardino MA. Recent and forgotten aspects of visceral pain. Eur $J$ Pain 1999;3:77-92.

60. Wilder-Smith $\mathrm{CH}$, Schindler D, Lovblad K, Redmond SM, Nirkko A. Brain functional magnetic resonance imaging of rectal pain and activation of endogenous inhibitory mechanisms in irritable bowel syndrome patient subgroups and healthy controls. Gut 2004;53:1595-601. 
61. Elsenbruch S, Rosenberger C, Bingel U, Forsting M, Schedlowski M, Gizewski ER. Patients with irritable bowel syndrome have altered emotional modulation of neural responses to visceral stimuli. Gastroenterology 2010;139:1310-9.

62. Dorn SD, Palsson OS, Thiwan SI, Kanazawa M, Clark WC, van Tilburg MA, Drossman DA, Scarlett Y, Levy RL, Ringel Y, Crowell MD, Olden KW, Whitehead WE. Increased colonic pain sensitivity in irritable bowel syndrome is the result of an increased tendency to report pain rather than increased neurosensory sensitivity. Gut 2007;56:1202-9.

63. Rapps N, van Oudenhove L, Enck P, Aziz Q. Brain imaging of visceral functions in healthy volunteers and IBS patients. J Psychosom Res 2008;64:599-604.

64. Wilder-Smith $\mathrm{CH}$. The balancing act: endogenous modulation of pain in functional gastrointestinal disorders. Gut 2011;60:1589-99.

65. Ritchie J. Pain from distension of the pelvic colon by inflating a balloon in the irritable colon syndrome. Gut 1973;14:125-32.

66. Bouin M, Plourde V, Boivin M, Riberdy M, Lupien F, Laganiere M, Verrier P, Poitras P. Rectal distention testing in patients with irritable bowel syndrome: sensitivity, specificity, and predictive values of pain sensory thresholds. Gastroenterology 2002; 122:1771-7.

67. Delvaux M. Do we need to perform rectal distention tests to diagnose IBS in clinical practice? Gastroenterology 2002;122:2075-8.

68. Piche M, Arsenault M, Poitras P, Rainville P, Bouin M. Widespread hypersensitivity is related to altered pain inhibition processes in irritable bowel syndrome. Pain 2010;148:49-58.

69. van der Schaar PJ, Lamers CB, Masclee AA. The role of the barostat in human research and clinical practice. Scand J Gastroenterol Suppl 1999;230:52-63.

70. Nozu T, Kudaira M. Altered rectal sensory response induced by balloon distention in patients with functional abdominal pain syndrome. Biopsychosoc Med 2009;3:13.

71. Camilleri M, McKinzie S, Busciglio I, Low PA, Sweetser S, Burton D, Baxter K, Ryks M, Zinsmeister AR. Prospective study of motor, sensory, psychologic, and autonomic functions in patients with irritable bowel syndrome. Clin Gastroenterol Hepatol 2008;6:772-81.

72. Andresen V. Visceral sensitivity testing. Best Pract Res Clin Gastroenterol 2009;23:313-24.

73. Corsetti M, Cesana B, Bhoori S, Basilisco G. Rectal hyperreactivity to distention in patients with irritable bowel syndrome: role of distention rate. Clin Gastroenterol Hepatol 2004;2:49-56.

74. Sun WM, Read NW, Prior A, Daly JA, Cheah SK, Grundy D. Sensory and motor responses to rectal distention vary according to rate and pattern of balloon inflation. Gastroenterology 1990;99:1008-15.

75. Mayer EA, Bradesi S, Chang L, Spiegel BM, Bueller JA, Naliboff BD. Functional GI disorders: from animal models to drug development. Gut 2008;57:384-404.

76. Park JH, Baek YH, Park DI, Kim HJ, Cho YK, Sohn Cl, Jeon WK, Kim BI, Rhee PL. Analysis of rectal dynamic and static compliances in patients with irritable bowel syndrome. Int J Colorectal Dis 2008;23:659-64. 
Methods to assess visceral hypersensitivity in irritable bowel syndrome

D. Keszthelyi, FJ. Troost, AA. Masclee

American Journal of Physiology-Gastrointestinal Liver Physiology $2012 \mathrm{Jul}$ 15;303(2):G141-54

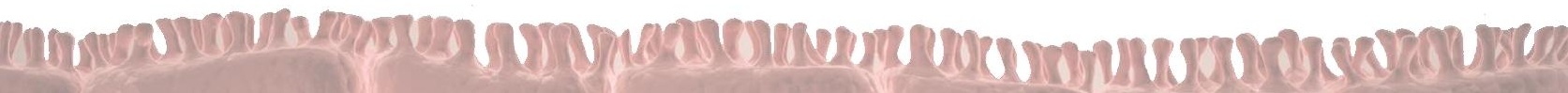




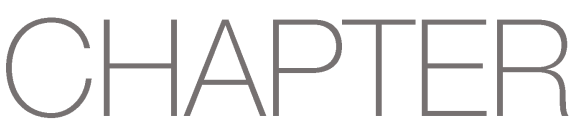

Methods to assess visceral hypersensitivity

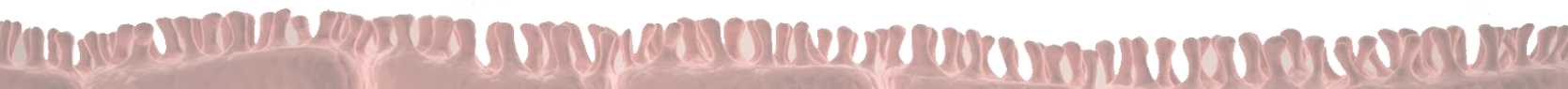




\section{ABSTRACT}

Irritable bowel syndrome (IBS) is a common functional gastrointestinal disorder, characterized by recurrent abdominal pain or discomfort in combination with disturbed bowel habits in the absence of identifiable organic cause. Visceral hypersensitivity has emerged as a key hypothesis in explaining the painful symptoms in IBS, and has been proposed as a 'biological hallmark' for the condition. Current techniques of assessing visceral perception include the computerized barostat using rectal distensions, registering responses induced by sensory stimuli including the flexor reflex and cerebral evoked potentials, as well as brain imaging modalities such as functional magnetic resonance imaging and positron emission tomography. These methods have provided further insight into alterations in pain processing in IBS, although the most optimal method and condition remain to be established. In an attempt to give an overview of these methods, a literature search in the electronic databases Pubmed and MEDLINE was executed using the search terms 'assessment of visceral pain/visceral nociception/visceral hypersensitivity, 'irritable bowel syndrome.' Both original articles and review articles were considered for data extraction. This review aims to discuss currently used modalities in assessing visceral perception, along with advantages and limitations, and aims also to define future directions for methodological aspects in visceral pain research. Although novel paradigms such as brain imaging and neurophysiological recordings have been introduced in the study of visceral pain, confirmative studies are warranted to establish their robustness and clinical relevance. Therefore, subjective verbal reporting following rectal distension currently remains the best-validated technique in assessing visceral perception in IBS. 


\subsection{INTRODUCTION}

Irritable bowel syndrome (IBS) is a common gastrointestinal disorder, affecting 10-15\% of the population in developed countries. Referred to as a functional gastrointestinal disorder, it is characterized by lower abdominal discomfort or pain with disturbed defecation in the absence of apparent structural or biochemical abnormalities that might explain the symptoms. ${ }^{1}$ Despite being very common, the pathophysiology of IBS is incompletely understood, which poses problems in the search for effective therapeutic approaches. Nonetheless, it is now widely accepted that an altered visceral sensitivity through abnormal endogenous pain processing plays an important role in the pathogenesis of IBS. ${ }^{2}$ Both central and peripheral mechanisms have been suggested to be involved in the development of pain symptoms. ${ }^{3}$ Several studies have provided evidence that IBS is associated with a dysregulation of the brain-gut axis, with peripheral sensory alterations dominating in some patients and disturbed central processing dominating in others. ${ }^{4}$ As the treatment and management strategies deployed to ameliorate these different aberrant mechanisms are distinct (i.e. pharmacological vs. psychological therapy), identifying the predominant underlying pathology remains an important clinical and therapeutic objective. Several tests have been developed over the past decades to assess and quantify visceral perception in IBS. The majority of current knowledge about visceral perception, however, is largely dependent on studies using subjective perception threshold determination and identifying altered cortical activity using neuroimaging, with far less rigorous and validated experimental paradigms than used in the area of somatic research. This review aims to give an overview of these methods, highlighting the advantages and limitations, and to define future directions for visceral pain research. 
6.2. ALTERED VISCERAL PAIN PROCESSING IN IBS: WHERE DOES IT GO WRONG? - A THEORETIC OVERVIEW

\section{PERIPHERAL AFFERENT PATHWAYS}

Innervation of the $\mathrm{Gl}$ tract from the esophagus through the transverse colon is provided by vagal afferents originating from the nodose ganglia and projecting centrally to the nucleus of the solitary tract. Vagal afferents are therefore important in the sensory innervation of the upper gastrointestinal tract, in particular the esophagus and stomach ${ }^{5}$ and are believed to mediate non-noxious physiological sensations, such as satiety and nausea. ${ }^{6}$ Vagal innervation decreases down the length of the $\mathrm{Gl}$ tract and is sparse in the distal colon. The more distal parts of the lower bowel are innervated by pelvic nerve afferents, originating from the sacral dorsal root ganglia and projecting to the sacral spinal cord. Besides, afferent innervation is provided throughout the entire GI tract by the splanchnic nerves projecting to T5-L2 segments of the spinal cord. More specifically, colonic afferents fibers project both to pelvic and splanchnic nerves. Pelvic afferents innervate the rectum, whereas splanchnic afferents innervate the distal colon and extend more proximally.7, 8 In general, the pelvic pathway is a predominantly low threshold pathway, 7,9 responding to low intensity of mechanical stimulation, whereas the splanchnic pathway is predominantly a high threshold pathway. ${ }^{7}$ Recently, Kyloh et al. demonstrated that the sensory innervation of the distal colon and rectum is significantly greater than the mid-proximal colon, probably due to additional afferent nerve pathways recruited for the defecation reflex. ${ }^{10}$

Both the pelvic and splanchnic systems carry afferents, which can be functionally divided into different subclasses:

> Mucosal afferents respond exclusively to fine tactile stimuli on the luminal surface. The majority of these afferents are also chemosensitive ${ }^{11}$ but have not been demonstrated to exhibit sensitization to chemical stimuli.

> Muscular afferents respond to distension at physiological levels $(<20 \mathrm{mmHg})$ and provide input for the perception of distension or contraction. Muscular-mucosal 
afferents respond both to tactile and distension stimuli (both noxious and physiological). These afferents predominate in the pelvic pathway. ${ }^{7}$

> Serosal and mesenteric afferents respond to noxious levels of distension (>30 mmHg). They detect overdistension and high amplitude contractions. ${ }^{12}$ These afferents are most commonly found in the splanchnic pathway.

> Mechanically insensitive afferents have the ability to acquire mechanosensitity in inflammatory conditions and generate spontaneous activity in the noxious range. ${ }^{13}$ On average, about $25 \%$ of pelvic and splanchnic afferents are mechanically insensitive..$^{13}$ It is also important to acknowledge, however, that not all mechanically insensitive afferent are able sensitize to mechanical stimuli. ${ }^{14,15}$

Sensitization of these distal peripheral afferents has been identified as an important mechanism in visceral hypersensitivity in IBS.16 The important question arises which afferents can in fact potentially contribute to altered visceral perception. Hughes et al. demonstrated that high-threshold mesenteric and serosal afferents of the splanchnic pathway show sustained sensitization following mild transient inflammation. ${ }^{12}$ These afferents also exhibit chemosenstivity to a various number of mediators, including bradykinin and ATP,14 providing evidence for a role of these afferents in postinflammatory hypersensitive conditions.

On the other hand, a significant proportion of mechanically insensitive afferents in colorectal pelvic and lumbar splanchnic pathways in mice have been demonstrated to acquire mechanosensitivity, albeit short lasting ( 20 minutes), under brief inflammatory conditions. Also, following treatment with zymosan resulting in long-lasting visceral hypersensitivity, a switch from mechanically insensitive phenotype to mechanically sensitive serosal phenotype was seen in a significant number of pelvic afferents. ${ }^{17}$ In fact, mechanically insensitive afferents in the pelvic nerve are more likely to acquire such mechanosensitivity than their splanchnic counterparts. ${ }^{13}$ This suggest a superior role for these 'silent' afferents - in particular in the pelvic nerve - in conditions of visceral hypersensitivity, which is supported by the observation that visceromotor responses to 


\section{Chapter 6}

noxious colorectal distension are absent after transection of the pelvic pathway but remain unaffected after transection of the splanchnic pathway. ${ }^{10}$ However, other studies were unable to find an increase in the total number of afferents showing firing activity following inflammation, ${ }^{12}$ which would indicate recruitment of these otherwise mechanically insensitive afferents. This suggests that post-inflammatory hypersensitivity is probably explained based on the existing population of afferents. Therefore, the role of 'silent' afferents in sustained visceral hypersensitivity remains to be further elucidated.

Overall, the selective sensitization of a single nociceptive pathway seems unlikely in IBS. First, IBS patients often complain of bloating, cramps, urgency and discomfort in addition to experiencing pain, suggesting the involvement of multiple or multi-modal afferent pathways. Second, IBS patients experience pain for long periods of time, which cannot be related to ongoing luminal distension at noxious levels. Third, nociceptor activation alone may not be sufficient to cause chronic pain or discomfort, suggesting the importance of other - in particular central - modulatory factors.

\section{SPINAL CORD}

Visceral afferents constitute less than $10 \%$ of all spinal afferent input. ${ }^{18}$ Upon entering the spinal cord, visceral afferents terminate in spinal cord laminae I, II, V and $X^{19}$ and converge onto spinal neurons in the lumbosacral and thoracolumbar segments.

In the spinal cord, two major ascending pathways have been proposed to be involved in visceral nociception: the anterolateral and the dorsal column pathways (see Figure 1). Ascending pathways of the anterolateral quadrant, most importantly the spinothalamic tract, have been proposed to conduct nociceptive information of visceral origin, besides transmission of signals evoked by noxious cutaneous stimuli. Spinothalamic projections are largely to the ventral posterior lateral (VPL) and ventral posterior inferior (VPI) nuclei of the thalamus, which in turn project to primary (SI) and secondary (SII) somatosensory cortices. These pathways transmit information regarding the intensity, duration, and location of noxious stimuli. However, unlike somatic sensation, which has strong 
homuncular representation in the primary somatosensory cortex, visceral representation is more diffuse. This could be the explanation for visceral sensation being poorly localized in comparison to somatic sensation.

Apart from projection to ventral posterior thalamic nuclei, spinothalamic afferents have been shown to connect with the medical thalamus at the level of the nucleus submedius. ${ }^{20}$ This region has been consistently shown to be activated in brain imaging studies. ${ }^{21}$ From these nuclei, ascending pain signals spread bilaterally to the prefrontal cortex (PFC), including the anterior cingulate cortex (ACC). ${ }^{18}$ In addition, there are projections to the periaqueductal gray matter (PAG), which has reciprocal connections with the medial thalamic nuclei and receives a direct input from the ACC. Neural terminations arising from medial thalamic nuclei are ideal for providing motivational and affective qualities associated with noxious stimulation, which should be activated by visceral stimuli that have a dominant affective component.

The spinoreticular tract, another constituent of the anterolateral system, conducts sensory information from the spinal cord to the reticular formation in the brainstem (see Figure 1). The reticular formation is mainly involved in the reflexive, affective and motivational properties of such stimulation. ${ }^{22}$ Third-order reticulothalamic tract neurons project from the dorsal and caudal medullary reticular formation to the medial and intralaminar nuclei of the thalamus. The spinomesencephalic tract, yet another component of the anterolateral system, ascends the spinal cord with fibers to various regions in the brain stem, including the PAG, locus coeruleus, and dorsal reticular nucleus in the medulla. ${ }^{23}$

Early studies have demonstrated that sensation of visceral distension was retained following extensive anterolateral chordotomy, ${ }^{24}$ lesioning the anterolateral afferent system, suggesting the existence of an alternative spinal ascending nociceptive pathway. Although the dorsal column system has traditionally been viewed as a pathway responsible for the discriminative aspects of tactile sensations and for kinesthesia, more recent studies have in fact identified the dorsal column as being of superior importance 


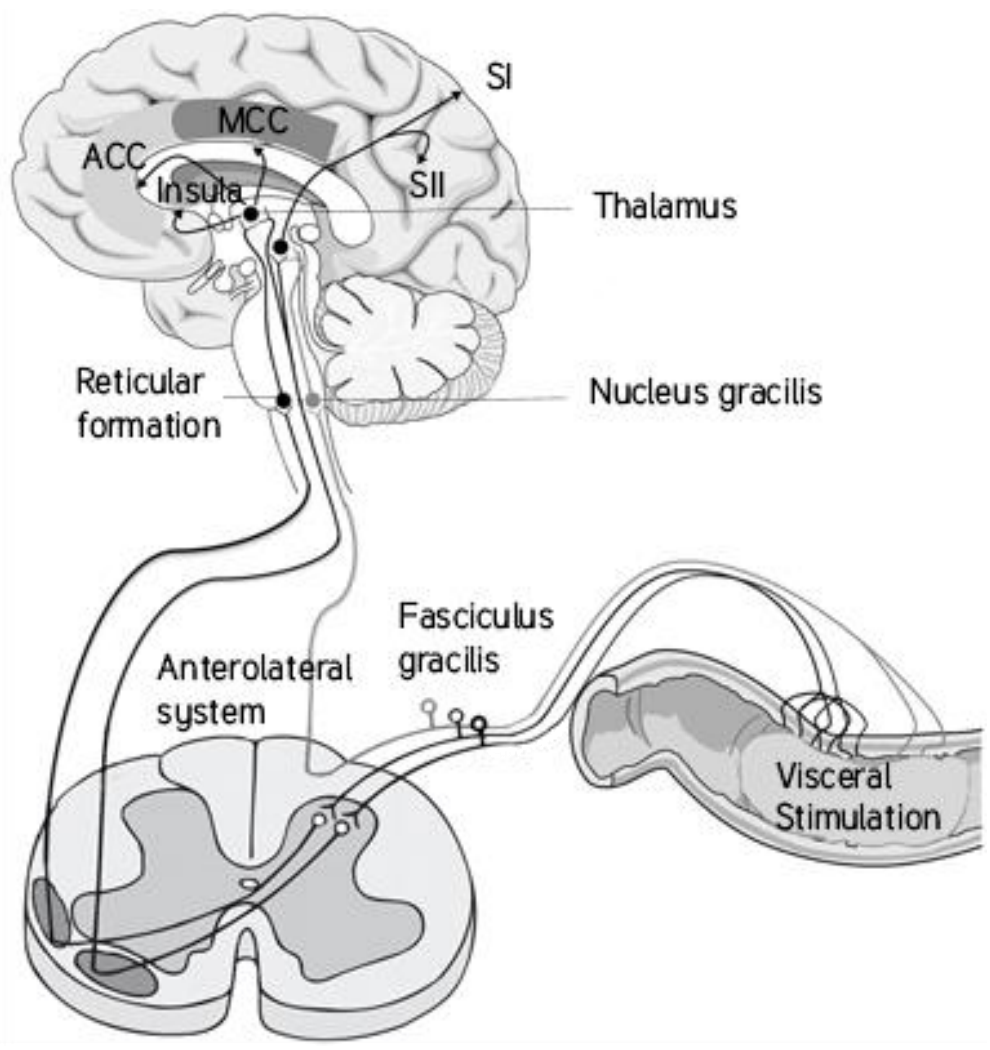

Figure 1: Schematic overview of ascending pathways involved in visceral nociceptive signaling. $\mathrm{ACC}=$ anterior cingulate cortex, $\mathrm{MCC}=$ mid-cingulate cortex, $\mathrm{SI}=$ primary somatosensory cortex, S\|l = secondary somatosensory cortex. The anterolateral system includes

in visceral nociception compared to spinothalamic and spinoreticular tracts. ${ }^{25} \mathrm{~A}$ dorsal column lesion in monkeys has been demonstrated to reduce the response of VPL neurons previously excited by colorectal distension. ${ }^{26}$ Furthermore, subpopulations of neurons within the dorsal horn have also been demonstrated to project to the lateral parabranchial nucleus and the PAG, ${ }^{25}$ supporting their role in nociceptive processes. Whichever spinal pathway is used to transport afferent input, from the thalamus, information is conveyed to cortical areas involved in sensory processing or those involved in processing emotional or affective information. ${ }^{27}$ 


\section{CORTICAL PAIN PROCESSING}

Because pain is a complex, multifactorial subjective and conscious experience, a large distributed brain network is accessed during nociceptive processing. A variety of regions identified as the 'homeostatic afferent network' (previously referred to as the 'central pain matrix'), consisting mainly of the posterior insula (primary interoceptive cortex), the anterior mid-cingulate cortex (aMCC) and the thalamus, have been found to be consistently activated in response to visceral stimuli.28, 29 This network is primarily responsible for pain processing, but also mediates affective, motivational, and motor aspects of a certain noxious stimulus. The single most consistent activation in brain imaging studies investigating visceral pain was found in the insular cortex, which is involved in integration of somatic and visceral information (posterior insula) and its affective component (anterior insula). Furthermore, lower gastrointestinal stimulation has been consistently shown to activate the anterior portions of the MCC, having direct connections with limbic and brain stem structures. ${ }^{21}$

Besides the homeostatic afferent network, brain imaging studies have also demonstrated a greater activation of the emotional arousal network following painful visceral stimuli, including the locus coeruleus complex, amygdala, hypothalamus, parahippocampal gyrus, perigenual anterior cingulate cortex (pACC), the anterior insula, and orbitofrontal cortex.21 This arousal network is engaged in the emotional processes modulating visceral responses, such as anticipatory anxiety or fear. The greater engagement of the emotional arousal network may also play a role in central pain amplification and is consistent with a model of IBS characterized by increased stress, anxiety, vigilance, and altered autonomic responses.

More specifically, anterior activation of MCC extending into the superior portion of the pACC has been reported in studies of non-nociceptive pain, eg. anticipated, imagined or empathy-related pain. ${ }^{30}$ The pACC is considered to be the primary cortical region in the descending pain inhibition system, with a high concentration of opioid receptors ${ }^{31}$ and connections to the PAG. ${ }^{32}$ IBS patients demonstrating hypersensitivity to rectal distension 
in fact showed reduced deactivation in this region compared to normosensitive IBS patients and healthy controls, suggesting alterations in descending modulatory nociceptive control. ${ }^{33}$ Also, Berman et al. showed that in IBS patients, alterations during cued expectation of an aversive rectal stimulus correlated with subsequent brain responses to the delivered stimulus in the PACC. Also, IBS patients have been shown to engage the anterior insula ${ }^{34}$ and the thalamus ${ }^{35}$ to a greater extent during pain expectation. The greater activation of these regions is consistent with inappropriate engagement of descending pain facilitatory mechanisms triggered by the prediction of an aversive stimulus and this has been interpreted as a possible prediction bias related to the expected pain intensity. ${ }^{34}$

\section{DESCENDING CONTROL OF PAIN PERCEPTION}

Neural circuits have been described originating from supraspinal sites that influence nociceptive activity in the spinal cord and primary afferents. The function of this supraspinal pain control is to either amplify or subordinate noxious or potentially noxious stimuli in coordination with the individual's homeostatic needs. ${ }^{36}$ Key component of this descending system is the PAG. Stimulation of this area leads to selective repression of pain, leaving other sensory modalities intact, an effect, which appears to be mediated via both the presynaptic inhibition of primary afferent input into the dorsal horn and the inhibition of dorsal horn projection neurons. ${ }^{37}$ The PAG receives input from collaterals of ascending fibers as well as from higher cortical centers such as the frontal neocortex, the pACC, the central nucleus of the amygdala, hippocampus and hypothalamus. Output of the PAG is to the rostroventral medulla and the dorsolateral pontine tegmentum. These two regions project to spinal cord dorsolateral funiculus and selectively target the dorsal horn laminae that accommodate nociceptive relay neurons. This circuit can therefore selectively modulate nociceptive transmission by acting on primary afferent nociceptive terminals and dorsal horn neurons responsive to nociceptive stimulation. ${ }^{38}$

Some neurons of the dorsal horn of the spinal cord are strongly inhibited when a noxious stimulus is applied to any part of the body, distinct from their excitatory receptive fields. 
This phenonemon is termed 'diffuse noxious inhibitory control' (DNIC). ${ }^{39}$ Accordingly, acute aversive heterotopic stimulation provides a temporary relief on chronic and recurrent pain. DNIC paradigms, such as the RIII reflex (see below), typically involve measurement of the nociceptive threshold for a 'test' stimulus before, during and after application of a second noxious 'conditioning' stimulus to an anatomically remote area of the body. DNIC have been proposed to be triggered by ascending spinoreticular fibers, with the medullary reticular formation playing a crucial role in the subsequent descending modulation of spinal nociception. ${ }^{40}$

More recently, it has become evident that descending pathways do not only suppress, but can also facilitate nociception. ${ }^{41,42}$ Overall, studies of visceral perception have demonstrated that nociceptive and anti-nociceptive information is processed in parallel. This is particularly true for supraspinal sites, where evidence of nociceptive-specific neurons is rare and evidence of supraspinal nociceptive-specific nuclei is nonexistent. ${ }^{43}$ Furthermore, current studies have not been able to delineate distinct inhibitory and facilitatory pathways, but rather indicate that the modulatory nociceptive balance appears to be distributed over the same functional circuits. ${ }^{41}$

\subsection{ASSESSMENT OF VISCERAL NOCICEPTION IN IBS}

\section{GENERAL PRINCIPLES}

The complexity of visceral nociceptive processes, as illustrated above, has imposed a challenge in assessing visceral perception in IBS. Traditionally, visceral perception has been tested by delivering a certain controlled sensory stimulus and measuring the nociceptive response evoked by it (see Figure 2). The gastrointestinal (GI) tract contains afferents that encode a wide range of intensities and modalities of stimulation including electrical, mechanical, thermal and chemical (including nutrient) stimuli. Choosing the correct stimulation method or interpreting differential responses to multiple stimuli may be of superior relevance in delineating pathological mechanisms. For instance, electrical stimulation is often thought of as non-physiological and therefore of limited use in 
assessing GI sensation. Furthermore, manipulation of the environment in which a sensory stimulus is given, pre- and post-nutrient infusion, or heterotopic stimulation for instance, can yield important physiological and pathophysiological information above and beyond that gained purely from quantitative sensory testing alone. With respect to the assessment of visceral perception, perhaps the most important aspect is the objectivity and robustness of the measuring the magnitude of the pain response, used as primary end-point for the experiment. Measurement of the pain response using verbal scores and scales to assess perceptive intensity and thresholds of nociception is largely influenced by subjective components, making it prone to reporting bias. Brain imaging and neurophysiological measurements, on the other hand, theoretically offer a more objective assessment of nociceptive responses. However, as will be illustrated later, these outcome measures are also greatly affected by cognitive-affective mechanisms, posing a challenge on the search for a surrogate marker of altered visceral perception in IBS. 


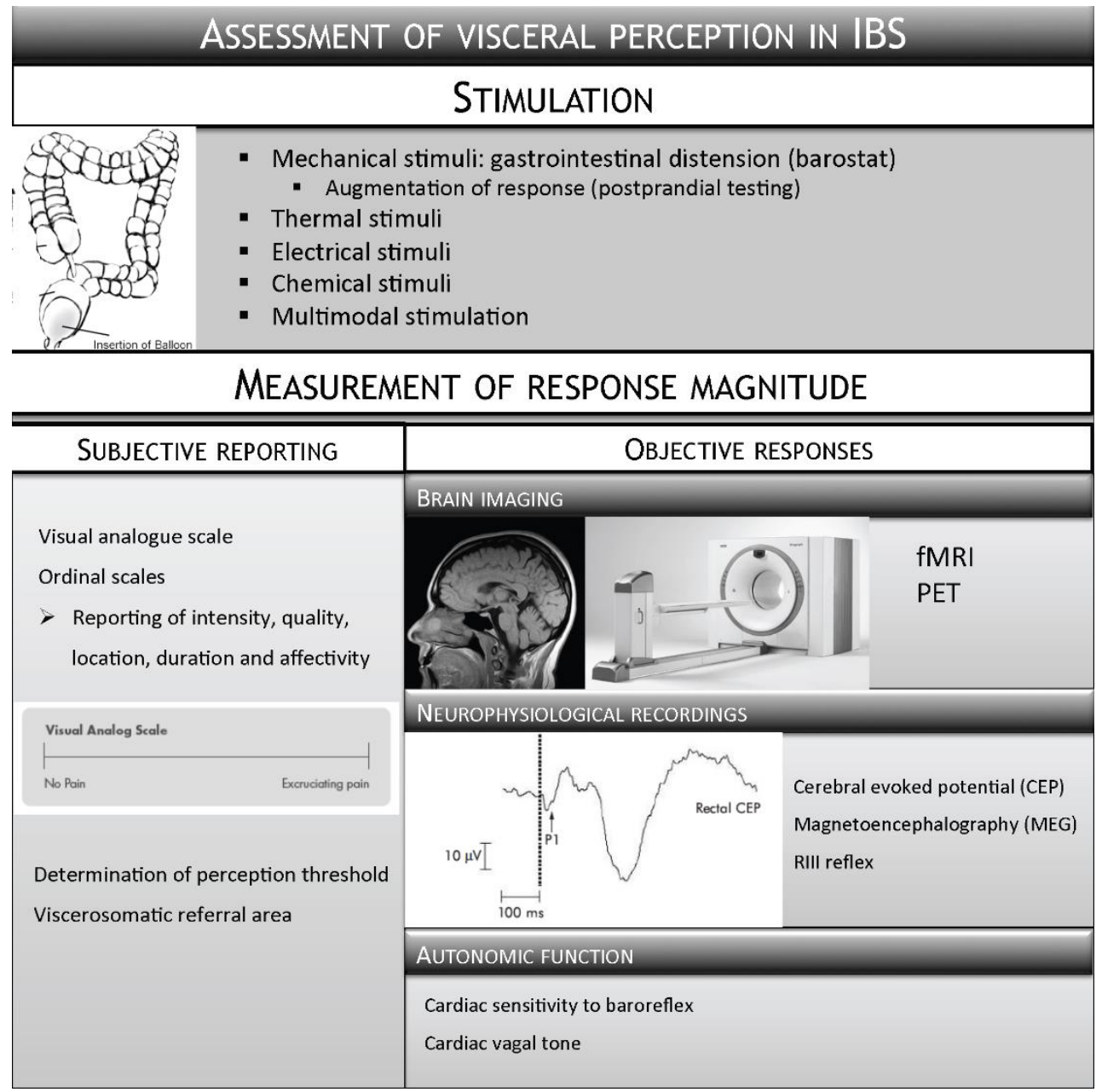

Figure 2: Schematic overview of assessment of visceral perception in irritable bowel syndrome (IBS). $\mathrm{fMRI}=$ functional magnetic resonance imaging. PET = positron emission tomography.

\section{MECHANICAL STIMULI: RECTAL DISTENSION}

Until recently, employing gut distensions has most widely been used to test visceral perception in IBS. Previous studies indicate that 35-60\% IBS patients exhibit increased visceral sensitivity to rectal balloon distension and that this hypersensitivity could be a biological marker of IBS.2, 44-46 The distal colon has also been used as an anatomic location 
to assess visceral perception. Both rectal and sigmoid distensions have been employed, although the overwhelming majority of studies have applied rectal distensions. It appears that when phasic distensions are applied, only minor differences can be observed with regards to the visceroperceptive responses between rectal and sigmoid distensions. ${ }^{47,48}$ As rectal distension has emerged as an attractive and more easily reachable alternative, we will aim further to focus on the discussion of this technique. The recent observation that the rectum also contains more afferents makes the rectum indeed an ideal target in assessing visceral nociception. ${ }^{10}$

Rectal visceral sensitivity can be tested by using polyethylene bags in conjunction with a barostat. ${ }^{49}$ The barostat is a device that maintains a constant pressure within an airfilled polyethylene bag and can measure variations in rectal tone by recording changes in the intrarectal pressure and volume. Rectal distension can be done according to a certain protocol, during which different pressures are applied to the rectal wall. During this procedure, the intensity and quality of perception can be measured by means of rating scales.

Several different distention protocols have been applied to assess visceral perception in IBS. The lowered sensory thresholds seen in IBS has been shown to be elicited by rapid rates of rectal distension, while in the majority of patients, the perception threshold during slow rectal distension pressure is not different from normal control subjects. It has also been postulated earlier that rapid phasic distensions preferentially stimulate serosal afferents. ${ }^{50}$ These afferents are particularly chemosensitive to inflammatory and enteroendocrine mediators such as serotonin, which suggests a role for nociception in conditions in which sensitization of visceral afferents is involved. ${ }^{12}$ Accepting that these afferents are at least in part responsible for the development of visceral hypersensitivity in IBS, rapid rectal distensions theoretically offer an ideal tool to assess perceptional status in these patients. Furthermore, complex distension protocols with non-random presentation of stimuli such as double random staircase distension reduce psychological response bias by making the stimulus unpredictable to the subject, ${ }^{51}$ which is desirable 
considering that IBS patients may be particularly prone to such bias due to anticipatory anxiety. However, these complex protocols usually require more distension levels and increasing the number of interrogations about sensations may introduce bias or errors in a fatigued and stressed or disinterested participant.

\section{MEASUREMENT OF PERCEPTIONAL RESPONSE}

During rectal distension, several parameters of visceral sensitivity can be collected to assess perceptional status. These include perceived intensity of the stimulus, extent of the viscerosomatic referral area and the threshold at which different sensations (pain, discomfort, urge) appear during the distension protocol. ${ }^{52}$ These components of visceral perception are not uniformly used by various groups, making comparison of study results troublesome. Standardization of testing procedures is therefore highly desirable, such as application of similar distension protocols and definitions in visceroperception assessment.

As for the intensity of perception, different scales including numeric rating scales, verbal rating scales, verbal descriptor scales, magnitude estimation and visual analogue scales (VAS) are employed. VAS has emerged as having psychometric properties that are superior to the other pain scaling methods because they fulfill multiple criteria for ideal pain measurement and assessment. ${ }^{53}$ These criteria include ratio scale properties, high test-retest reliability and repeatability, the capacity to detect small differences, internally consistent measures of clinical and experimental pain, sensitivity to variables that increase or decrease pain, detection of individual differences in pain sensitivity and ease of use. ${ }^{54}$

The majority of the research groups investigating visceral perception using the barostat techniques have focused on identifying the thresholds for perception in IBS patients as these have been shown to be lowered in comparison to healthy volunteers. However, threshold measurements of pain perception are limited as they fail to assess alterations in pain intensity that may occur over a wide range of noxious intensities. On the other 


\section{Chapter 6}

hand, investigation of the perceived intensity of the given stimulus as well as recording the viscerosomatic referral area can give extra information on processing of visceral stimuli.2, 52 In addition to lowered thresholds to visceral stimuli, marked alterations in somatic referral patterns in response to balloon distension of the colon have been described in patients with IBS. ${ }^{55}$ Atypical referral of internal sensation is a strong indication for altered spinal processing of visceral sensory information and is a manifestation of central sensitization. Therefore, assessment of viscerosomatic referral may have added value in determining the perceptional status in IBS.

Currently, distension paradigms differ in duration and type of inflation (tonic vs phasic), level of bias (ascending vs randomized distension) and measurement of response magnitude (perceptual threshold or rating). Despite the fact that not all patients exhibit hypersensitivity to rectal distensions, the barostat procedure appears to be a reliable and valid approach to assess visceral nociception. The barostat has been shown by several studies to be reproducible in a single subject for at least to testings, ${ }^{56-58}$ with the best reproducibility seen in higher pressure ranges $(36-48 \mathrm{mmHg}) .{ }^{59}$ This is underlined by the fact that the barostat has also shown robust changes following administration of a narcotic analgesic even is small crossover samples, further supporting the measure's validity for assessing pain perception.60, 61 . It is also important to note that these perceptual alterations were independent of changes in compliance or tone..$^{62}$ Evidence from crossover studies points to considerable reliability and validity for rectal distensions to testing visceral perception, reviewed extensively by Mayer et al. ${ }^{51}$ Furthermore, the barostat method in conjunction with verbal reporting has shown to have high sensitivity and specificity for detecting visceral hypersensivity in IBS patients $(95.5 \%$ and $71.8 \%$, respectively). ${ }^{44}$

However, given its subjective nature, rectal distensions in combination with verbal reporting can only be an indirect measure of the underlying neurophysiological processes. Due to the influence of cognitive and emotional factors, the relationship between visceral afferent sensitivity and conscious pain perception is highly non-linear. This could also 
account for the fact that numerous studies have found poor correlation between sensitivity testing by barostat procedure and IBS symptom severity. ${ }^{51,52,63-65}$

\section{AUGMENTATION OF THE SENSORY RESPONSE: POSTPRANDIAL VISCERAL PERCEPTION}

Visceral sensitivity studies are generally performed in the fasted state. However, bowel symptoms in IBS patients often exacerbate after consuming food. ${ }^{66}$ There is ample evidence that gastrointestinal nutrient content may either be the source or contribute to abnormal, painful, or uncomfortable visceral perception in IBS. ${ }^{67}$ Several groups have reported on the effect of nutrients on visceral sensitivity in IBS patients. Rectal and colonic perception thresholds in IBS patiens have been shown to be lowered following intraduodenal infusion of fat. 68,69 Furthermore, a liquid meal was shown to enhance rectal sensitivity in IBS, ${ }^{70-72}$ with a fatty meal producing more pronounced effect in comparison to a carbohydrate meal..$^{71}$ These results could represent normal nutrient sensing, with which rectal distensions interacts abnormally resulting in an augmented perceptional response. On the other hand, it may also suggest a malfunction in intestinal nutrient sensing resulting in increased visceral perception following a meal. It has been postulated that enteroendocrine cells in the intestinal mucosa respond to changes in luminal contents by releasing mediators such as cholecystokinin and serotonin (5-HT), which in turn activate specific receptors on primary afferent nerve terminals. Cholecystokinin, a peptide released in response to intake of fat, has been postulated to sensitize mechanoreceptors as it has been demonstrated to increase rectal nociception in healthy volunteers. ${ }^{73}$ Serotonin, on the other hand, could also be a mediator of meal-induced nociception as higher postprandial levels of $5-\mathrm{HT}$ have been reported in IBS patients. ${ }^{74}$ Furthermore, Simrén et al demonstrated that antagonizing $5-\mathrm{HT}_{3}$ receptors reduces lipidinduced colonic hypersensitivity. ${ }^{75}$ Therefore, testing of visceral sensitivity in IBS patients in a postprandial state can also have an added value in assessing visceral perceptional status.

\section{NONMECHANICAL STIMULI: ELECTRICAL STIMULATION}

Besides mechanical stimulation, a number of other stimulation modalities have been used 


\section{Chapter 6}

to assess visceral sensitivity. Transmucosal electric nerve stimulation, for instance, induces perception by nonspecific stimulation of afferent pathways without the involvement of a specific receptive unit. Transmucosal electrical stimulation of the jejunum in healthy controls and IBS patients resulted in subjective sensations similar to those elicited by jejunal balloon distension. However, while IBS patients had lower sensory thresholds to mechanical stimulation of the small intestine compared to controls, thresholds to electrical intestinal stimulation were similar in IBS patients and controls. On the other hand, altered viscerosomatic referral areas were seen in response to both types of jejunal stimulation. ${ }^{76}$ A decrease in sensory thresholds in IBS has also been demonstrated to electrical stimulation of the rectum. ${ }^{77}$ Disadvantage of using electrical stimulation is the nonspecific activation of nerve endings, resulting in neural discharge from nerves, which might not otherwise be activated during nociceptive processes.

\section{THERMAL STIMULI}

Thermal stimulation of the gut can be provided via intraluminal bags by recirculating water at adjusted temperatures. Thermal stimuli are also potentially applicable in conjunction with mechanical and electrical stimuli for the evaluation of visceronociceptive responses. It has been demonstrated that similarly to mechanical stimuli, IBS patients also demonstrate lower thresholds to thermal stimulation of the rectum. ${ }^{78}$

\section{CHEMICAL STIMULI}

Chemical stimuli have been used widely to study pain mechanisms. Capsaicin is commonly applied as a sensitizing stimulus in studies of somatic pain. Hammer et al. demonstrated that intestinal capsaicin infusion resulted in a sensation of warmth and burning, in addition to the generation of cramps. This cramping sensation was independent of changes in bowel motility or biomechanics and the authors concluded that capsaicin was acting directly on mechanosensitive afferents. ${ }^{79}$ In addition to capsaicin producing these unique sensory characteristics, it was also noted that the time to first sensation of a second capsaicin infusion was significantly reduced suggesting the induction of peripheral sensitization of capsaicin-sensitive afferents. ${ }^{79}$ Capsaicin has therefore also been 
successfully used to evaluate chemical hypersensitivity in functional dyspepsia. ${ }^{80}$ On the other hand, drawbacks of chemical stimuli include relative long latency time to onset of effects, in comparison to other stimulation modalities, and that these effects are often not reproducible. ${ }^{81}$ Therefore, validation of results using chemical stimulation in IBS is still warranted.

\subsection{BRAN IMAGING}

In the past decade, accumulating evidence on visceral nociceptive processes has emerged from results using brain imaging studies. Brain imaging techniques rely on the principle that increased neuronal activity within the brain is associated with increased metabolism and regional cerebral blood flow. Comparison of images recorded during resting and active periods following administration of a nociceptive stimulus, for instance rectal distension, reveals regions of increased cortical activity and these measures have been used to increase understanding of the functional properties of the brain.

\section{FUNCTIONAL MAGNETIC RESONANCE IMAGING (FMRI)}

The most commonly used brain imaging entity, fMRI, is based on the difference in the magnetic properties of oxygenated and deoxygenated blood, specifically the difference in decay time of the MR signal from oxyhemoglobin and deoxyhemoglobin, referred to as the blood oxygenation level dependent (BOLD) technique. This difference in signal characteristics allows for the localized detection of regional cerebral blood flow (rCBF). These regional changes in blood flow, volume and oxygenation of haemoglobin derive from changes in neuronal activity and, thus, regions of activation may be identified by subtracting regional cerebral blood flow during a control condition from blood flow during a stimulus condition or by correlating regional blood flow with the intensity or time course of a stimulus or its perception. ${ }^{82} \mathrm{~A}$ major advantage of $\mathrm{fMRl}$ is that it is non-invasive and non-cumulative, allowing subjects to be studied repetitively. fMRI has an excellent spatial resolution (2-5 mm), especially in the more superficial layers. Limitations are seen in the deeper structures, such as the brainstem and thalamus, due to pulsation artifacts. The 


\section{Chapter 6}

temporal resolution is poor (1-3 s) and therefore fMRI is not a specific tool for investigating the neuronal activity directly related to the painful stimuli. Since the exogenous brain activity takes place within the first 150 ms post stimulus, the response may miss the fast occurring activity and model, instead, the endogenous activity rather than brain responses due to pain.

\section{POSITRON EMISSION TOMOGRAPHY (PET)}

PET scanning utilizes biological molecules synthesized with radiolabeled isotopes, such as ${ }^{150}$-labeled water to monitor regional changes in cerebral blood flow. The most commonly used in visceral pain research is $\mathrm{H}_{2}{ }^{15} \mathrm{O}$. In the normal brain, rCBF is closely coupled to regional cellular metabolism, therefore ${ }^{15} 0$-labeled water serves as a reliable monitor of cerebral interneuronal activity. ${ }^{83}$ Alterations in rCBF can then be mapped to specific intracerebral structures and foci, using either MRI or a computerized stereotactical method. PET has excellent spatial resolution $(2-5 \mathrm{~mm}$ ) and allows the operator to tag important biological molecules that bind to targeted receptor groups or glucose metabolism in active neuronal tissue. PET is superior in imaging radiopharmaceuticals and/or other ligands as it offers the ability to study receptor distribution and explore the site of action. This also allows an insight into the organization of functional networks in the brain, which cannot be achieved merely morphological investigations or regional blood flow analysis. However, the temporal resolution of PET is poor (minutes), and as the subject receives a considerable dose of radiation, group analyses are needed for meaningful results, interpreting endogenous brain activity following pain rather than exogenous brain activity following painful stimulation. Another major disadvantage is the expense and limited availability of a PET scanner.

\section{BRAIN IMAGING TECHNIQUES: VALIDITY REMAINS TO BE CONFIRMED}

Several brain imaging studies have demonstrated altered activation patterns in IBS following rectal distension compared to health controls. ${ }^{21,29}$ Following initial excitement, given the relative novelty of the technique in studying visceral pain, a number of questions remain regarding the validity of these testing modalities. These modalities rely 
on observing between-scan or between-session differences that are attributed to changes in the experimental paradigm, for example measurements at baseline, during rectal distension or during anticipation of such stimulus. However, changes occurring in brain activity between sessions can be attributed to many other factors including nonsystematic variations in the scanner's acquisition characteristics, variations in head orientation, movement, and psychophysiological effects such as the level of arousal that can vary from session to session. ${ }^{84}$ In fact, Coen et al. demonstrated a decrease in perceived stimulus intensity due to habituation over three different sessions in healthy volunteers using esophageal stimulation by fMRI. ${ }^{84}$ Another study by Naliboff et al. examined perceptual and brain activity responses to rectal distensions in IBS and healthy controls using $\mathrm{H}_{2}{ }^{15} \mathrm{O}$ PET 6 times over a 12-month period. ${ }^{85}$ Interestingly, it was found that perceptual rating normalized over the 12 months of the study, while IBS symptom severity did not, indicating decreased vigilance. In response to rectal distention, stable activation of regions of the homeostatic afferent network (including thalamus and insula) was observed over the 12-month period, while activity in the emotional arousal network decreased. These findings indicate a differential habituation response to repeated rectal distensions during this testing procedure over these distinct functional networks.

A limited number of studies have evaluated brain activation responses to different treatments in IBS. Morgan et al. reported on the effect of low-dose amitriptyline on rectal distension-induced brain activation combined with a psychological stressor in 19 women with painful IBS using fMRI. Although decreased activation was demonstrated in the pACC when applying the psychological stressor, no effect of amitriptyline was found on symptoms or distension alone. ${ }^{86}$ Berman et al. reported on the effects of a 3-week alosetron treatment in 49 non-constipated IBS patients using $\mathrm{H}_{2}{ }^{15} \mathrm{O}$ PET. Treatment was associated with reduced blood flow in the amygdala, PFC and the pontine region, but not in the homeostatic afferent network. Furthermore, these changes were only observed at baseline and during expectation, not during rectal distension, suggesting an effect on the arousal network, rather than those associated with primary processing of sensory input. ${ }^{87}$ A small study by Lackner et al. investigated the effect of cognitive behavioral therapy in 
6 IBS patients using $\mathrm{H}_{2}{ }^{15} \mathrm{O}$ PET. Reduces regional flow in the pACC and the parahippocampal gyrus were observed, which was accompanied improvement in GI symptoms and psychological functioning..$^{88} \mathrm{~A}$ recent study demonstrated that treatment with neurokinin-1 antagonist resulted in reduced activation in the emotional arousal network, which was paralleled by an improvement in mood and pain rating. ${ }^{89}$ Similarly, normalizations between key regions of the emotional-arousal circuit in patients were shown following treatment with a CRF-1 antagonist during expectation of abdominal pain, in particular in patients with high level of anxiety.90 Although studies using classical analgesics are still lacking, it appears that brain imaging is more sensitive in detecting changes in the emotional-arousal network, responsible for modulatory effects of pain sensation, rather than in the homeostatic afferent network. However, additional studies will be necessary to confirm the robustness of measuring brain activation patters to assess therapeutic efficacy in IBS, especially in patients with comorbid anxiety.

Another limitation of brain imaging studies is its poor correlation with symptoms. A recent fMRI study found significant alterations in brain activity patterns comparing normosensitive and hypersensitive IBS patients, even though no significant differences were observed between the two groups in terms of GI symptoms. ${ }^{33}$ This questions the fact whether changes in brain activity, as reflected by imaging studies, show any correlation with symptom severity.

\subsection{NEUROPHYSIOLOGICAL RECORDINGS TO ASSESS VISCERAL NOCICEPTION}

CEREBRAL EVOKED POTENTIAL (CEP)

CEPs are the electrical manifestation of the brain's response to an external stimulus. This technique involves the brief presentation of a sensory stimulus, which is time- and phaselocked to the recording of the electroencephalogram (EEG) via surface electrodes placed on the scalp. Stimulus-specific CEPs occur at a fixed time after each stimulus, while other brain activity occurs randomly. CEP amplitudes are typically lower than the amplitudes 
of spontaneous EEG; but the CEP amplitudes become higher during the averaging process, and most of the background noise cancels out. In order to extract the CEPs with a good signal-to-noise ratio, a number of stimulations are presented at a certain frequency, and these stimulation trials are then cleaned for artifacts and averaged. The main CEP peak components are termed P1, N1, P2, and N2. Neurophysiological convention describes negative potentials as upward deflections (N1 and N2) and positive potentials as downward deflections (P1 and P2). Each peak in the CEP represents a synaptic event associated with the synchronous transmission of afferent information from one group of neurons to another. Latency refers to time (in $\mathrm{ms}$ ) from the stimulus trigger to the peak component. Amplitude is defined as the voltage difference between consecutive CEP peaks. Early recorded CEP complex ( $\sim 100 \mathrm{~ms}$ ) represents stimulus-specific activation of the primary or secondary somatosensory cortex and insula and depends on transmitted afferent signal strength intensity. P1 latency represents the time interval between stimulation and activation of these regions. In healthy subjects, as the perceived stimulus intensity increases, there is a concurrent reduction in latency and an increase in amplitude of the CEP components. ${ }^{91}$ Cortical activity of $\sim 250$ ms or more after visceral stimulation reflects brain endogenous processing of the afferent information and represents a more general 'arousal'-type response. Hobson et al.92 established the following relationship between CEP morphology and pain thresholds: 1) Afferent hypersensitivity refers to pain perception at relatively low stimulus intensity with a normal-latency P1 component. 2) Hypervigilance refers to pain perception at low stimulation intensity, but with prolongedlatency P1 responses (indicating that afferent signaling pathways are not sensitized, instead patients were "amplifying" normal-intensity stimuli once the stimulus reached the brain). 3) Hyposensitivity refers to maximum stimulus application (limited by the stimulator and safety reasons) without eliciting a normal CEP response. On this basis, it has been proposed that CEP can neurophysiologically distinguish non-cardiac chest pain patients using esophageal stimulation into altered afferent transmission and increased secondary processing. ${ }^{92}$ More recently, IBS patients were also characterized according to their neurophysiological profile by recording CEPs following rectal stimulation. An overwhelming majority (49\%) had normal pain threshold and normal afferent sensory 


\section{Chapter 6}

transmission. Only a minority (24\%) of the patients afferent hypersensitivity, $12 \%$ were hypervigilant and $15 \%$ were even hyposensitive to rectal electrical stimuli. ${ }^{93}$ This study, however, did not assess the relationship of CEP responses to IBS symptom severity.

Other groups $94-96$ have observed shorter latencies in IBS patients compared to healthy controls. This was further shortened following meal intake ${ }^{96}$ or thermal stress (drinking cold water) ${ }^{94}$ in IBS patients, whereas no effect was observed in healthy controls. This decreased latency was suggested to be caused by an increase in the number of rectal afferents recruited and their higher conduction. However, no evidence exists that the conductance velocity of these otherwise silent afferents would differ from the preexistent population of afferent nerves. These silent afferents could rather account for the larger amplitude of the evoked potentials observed in IBS. 95

It is also to be noted that there are considerable discrepancies between the studies that have employed CEP, which makes comparison somewhat troublesome. This is probably due to methodological differences. Both electrical and mechanical stimuli have been used to generate neural responses. Electrical stimulations have the advantage that they allow precise latency measurements; in case of balloon distension, the responses are preceded by a variable latency of $50 \mathrm{~ms}$ due to balloon inflation. On the other hand, electrical stimuli can provoke contractile activity during and after stimulation, which causes increased variability in the responses recorded.

Overall, CEP is an intriguing method to assess visceral perception as it has excellent temporal resolution and potentially allows discrimination between peripheral and central components of nociception. Furthermore, CEPs recorded following rectal distension are perfectly reproducible in the same subject. ${ }^{97,} 98$ Although surrogate measurements of visceral pain can be obtained from other visceral function studies, such as barostat or metabolic imaging techniques, evaluation of pain using these tools has several limitations, including anticipation, habituation and lack of non-stimulus-specific activity. CEP, on the other hand, potentially provides an objective measurement of the pain response and does have the required temporal resolution, with early potentials indicative of peripheral 
afferent input and later potentials of central processing, thereby allowing discrimination of the origin of underlying pathology. However, knowledge on CEP responses in IBS is still limited. It remains to be established, furthermore, which parameter of CEP recordings (e.g. peak latency or amplitude) are of clinical relevance, in particular in terms of detecting visceral hypersensitivity and defining response to a certain therapy. ${ }^{99}$ Validation of the technique is warranted to legitimize its role in assessing visceral perception, such as definition of the relation of the CEP response to symptom severity. Currently, research involving registration of CEP is being practiced in only a few laboratories and further research is necessary to validate findings and address the observed discrepancies in results.

\section{MAGNETOENCEPHALOGRAPHY (MEG)}

MEG is a non-invasive neuroimaging modality that allows detection of cortical neuromagnetic activity arising from postsynaptic current flow in neurons, as opposed to metabolic changes, which are considered secondary markers of neural activity. Visceral stimulation leads to cortical activation that generates minute magnetic fields, which are $1 / 8$ of the magnetic field of the earth. ${ }^{100}$ These can be detected with highly sensitive sensors known as SQUIDS (super conduction quantum interference device), which are contained within a helmet-like apparatus that covers the subject's head. ${ }^{101}$ By mathematically modeling the topographic distribution of the magnetic field pattern on the scalp, the three-dimensional location of the neurons generating the signal can be inferred using mathematical models. As well as having comparable spatial resolution to PET and fMRI, MEG has a superior (millisecond) temporal resolution that permits a more dynamic interpretation of brain area activation after visceral stimulation. This latter quality and the technological advances allowing deeper regions of the brain to be studied offer the exciting prospect of providing a second by second evaluation of pain pathway activation and patterns of aberrant pain processing.

In CEP, the electrical signal recorded is distorted by the structures that lie between the cortical source and the recording electrode, making the localization of the neural 
correlates of the response difficult, therefore resulting in poor spatial resolution. Unlike the electrical signal recorded with CEP, in MEG the magnetic field generated by an active group of cortical neurons passes through these structures without attenuation. Because the early MEG responses are stimulus-specific, this type of approach has a greater potential for becoming a robust and objective outcome measure to study GI sensory processing. MEG is especially sensitive to the tangential activity in the cortex. A technical limitation of MEG is however that it is less able to resolve the radial current, which makes imaging in deeper regions of the brain troublesome. Magnetic fields generated by subcortical neurons are not readily detectable because, unlike cortical neurons, which are arranged in columns, many of the subcortical neurons are arranged in spherically symmetric manner so that the magnetic fields emitted are mutually cancelled. Currently, MEG is not widely available; systems are only present in specialist centers. Also, a very limited amount of studies have investigated visceral nociception using MEG, ${ }^{102}$ with no studies published to date in IBS.

\section{RECORDING SPINAL REFLEX RESPONSES: THE RIII REFLEX}

Apart from registering changes in neural activity using either brain imaging or EEG/MEG, assessment of reflex responses to noxious stimulation also offers an objective marker of spinal nociceptive processes, in particular. The flexion reflex, also known as the nociceptive withdrawal reflex and described first by Sherrington, ${ }^{103}$ is a polysynaptic and multisegmental spinal reflex that induces a complex flexion synergy of the ipsilateral limb following a cutaneous stimulus. The mechanical response is a rapid withdrawal movement that constitutes a protective mechanism against possible limb damage. The flexion reflex can be elicited experimentally by the electrical stimulation of a cutaneous sensory nerve (most often the sural nerve) and consists of the activation of an ipsilateral flexor muscle (usually the biceps femoris), which is recorded using standard electromyographic equipment. Hugon distinguished two excitatory components of this reflex having latencies of 40-60 ms (RII) and 85-120 ms (RIII) and explained these as due to activation of different cutaneous group A-fiber afferents (A-beta and A-delta, respectively). The RII reflex is a less stable and poorly investigated response, elicited at low non-nociceptive 
stimulation intensity. Therefore, the threshold of the Rll is significantly below the pain threshold and is not seen in all subjects. ${ }^{104}$ On the contrary, the RIII reflex, a widely investigated and ever-present response, is considered to correspond to the intensity of the stimulus (six- to seven-fold the detection threshold). ${ }^{105}$ Evidence exists that the threshold of the RIII reflex, defined as the average minimal current that elicited the reflex response, corresponds to the pain threshold and that the size of the reflex is related to the level of pain perception. ${ }^{106}$ Therefore, the RIII reflex has been proposed to be a useful tool in assessing nociception, free of the subjective nature of verbal reporting and subsequent report bias.

Several studies have investigated the influence of rectal distension on the RIII reflex in humans, offering further scope for studying the spinal projections from viscera afferents and their correlations with the nociceptive pathways. Bouhassira et al. showed that rapid rectal distensions facilitated the RIII reflex recorded from the lower limb, but at the highest distension level (i.e., $40 \mathrm{mmHg}$ ) facilitation was followed by inhibition. ${ }^{107}$ In contrast, slowramp distensions induced gradual inhibition of the RIII reflex that correlated with both the distension volume and the visceral sensation. It has been proposed that reflex inhibitions were probably related to the activation of spinal and/or supraspinal pain modulation systems, possibly through a DNIC-like mechanism. One plausible explanation for the facilitatory effects following rectal distension, which were not observed when the reflex was recorded at the upper limb, was that they were caused by the convergence of rectal and RIII afferents at the same levels of the spinal cord. Furthermore, the authors suggested that the opposite effects of rapid- and slow-ramp distensions on the RIII reflex could be due to the selective activation of different populations of rectal mechanoreceptors.

In a later study employing the same methodology in IBS patients, it was demonstrated that the RIII reflex was significantly facilitated during slow-ramp distension (as opposed to the inhibition seen in healthy controls). Inhibitions induced by rapid distensions were significantly reduced compared to healthy controls. ${ }^{108}$ These results suggest a failure of 


\section{Chapter 6}

descending antinociceptive control, leading to hyperexcitability of spinal nociceptive neurons in IBS. However, factors such as sustained attention, anticipation of pain, sustained muscle contraction, the type of which may occur when contracting the anal sphincter during rectal distension, can have either facilitatory or inhibitory effects on RIII and therefore these must be taken into when assessing nociceptive responses. Therefore, acceptance of the RIII reflex as a true surrogate marker will require additional conformation. Furthermore, with regards to assessment of responsiveness to drug treatment, initial studies investigating the effects of tegaserod using the RIII reflex have been reported 109,110 demonstrating reduction of the facilitatory effects of rectal distension on the RIII reflex in a selected group of IBS patients. However, effects of classical analgesics such as opioids have not yet been studied. It remains also to be elucidated which order of magnitude of inhibition of the Rlll reflex can translate into clinically relevant alterations in symptoms severity.

\subsection{MEASUREMENT OF AUTONOMIC DYSFUNCTION IN IBS}

Experimental data indicate that increased sympathetic activity magnifies perception of gut stimuli.111 In man, cardiac sensitivity to the baroreflex (CSB - afferent limb of the ANS) and cardiac vagal tone (CVT - efferent limb of the ANS) have been used to measure responses related to autonomic nervous system (ANS), reviewed recently by Mazurak et al. ${ }^{112}$ A study by van der Veek et al. revealed that IBS patients exhibited increased baseline CSB relative to healthy controls. ${ }^{113}$ CSB was also measured following visceral afferent stimulation using mild and intense rectal distension. During intense stimulation, the CSB increase observed following mild stimulation persisted in controls, whereas it declined to baseline in IBS. The authors suggested that this difference was due to altered information processing in the brainstem, more specifically in the nucleus of the solitary tract. More recently, Spaziani et al. were not able to reproduce the findings, showing lower CSB in IBS compared to controls. ${ }^{114}$ This can either be explained by the fact that changes in ANS function are not uniformly present in IBS or that the techniques in order to record these alterations are still suboptimal. A clear disadvantage of this approach, for instance, 
is that it requires off-line analysis of data acquired over a 60- to 90-second period, which limits the investigation of on otherwise dynamic regulatory process. This poorer temporal resolution masking fluctuations may result in a false impression of a solitary rise or drop in the ANS measure of interest. New advances in technology may give us the opportunity to expand on these experiments in a degree of detail not previously possible.

\subsection{MEASURING VISCERAL PAIN IN IBS: WHERE DO WE STAND NOW? - OPPORTUNITIES AND PITFALLS}

Currently, a considerable number of methods are employed to assess visceral perception in IBS (see Tables 1 and 2 for comparison of the different techniques). Over the past decades, rectal distensions in combination with verbal reporting has emerged as a robust, valid, practically applicable and widely accepted method to test visceral perception in IBS. Increased sensitivity to rectal distension has been suggested to represent a biological marker for IBS.2 Also, several additional approaches have been applied to increase the diagnostic sensitivity of the barostat, including meal stimuli.72 However, not all IBS patients represent this hypersensitivity to rectal distension. Furthermore, the invasive nature of this barostat method has somewhat withheld the widespread application of rectal distensions in clinical practice. Moreover, a number of recent observations have implicated that subjective reporting during rectal distension can largely be influenced by psychological factors, such as vigilance and anxiety. IBS patients have in fact been shown to have a psychological tendency to report urge and pain rather than increased neurosensory sensitivity. ${ }^{15}$ This makes the identification of patients who have true visceral hypersensitivity, without the interference of subjective reporting, especially challenging. This is considerably true in a patient group with a high incidence $(50-80 \%)$ of psychological disorders such as heightened anxiety, depression, somatization, dysthymia, and panic disorders have been reported. ${ }^{1}$ 


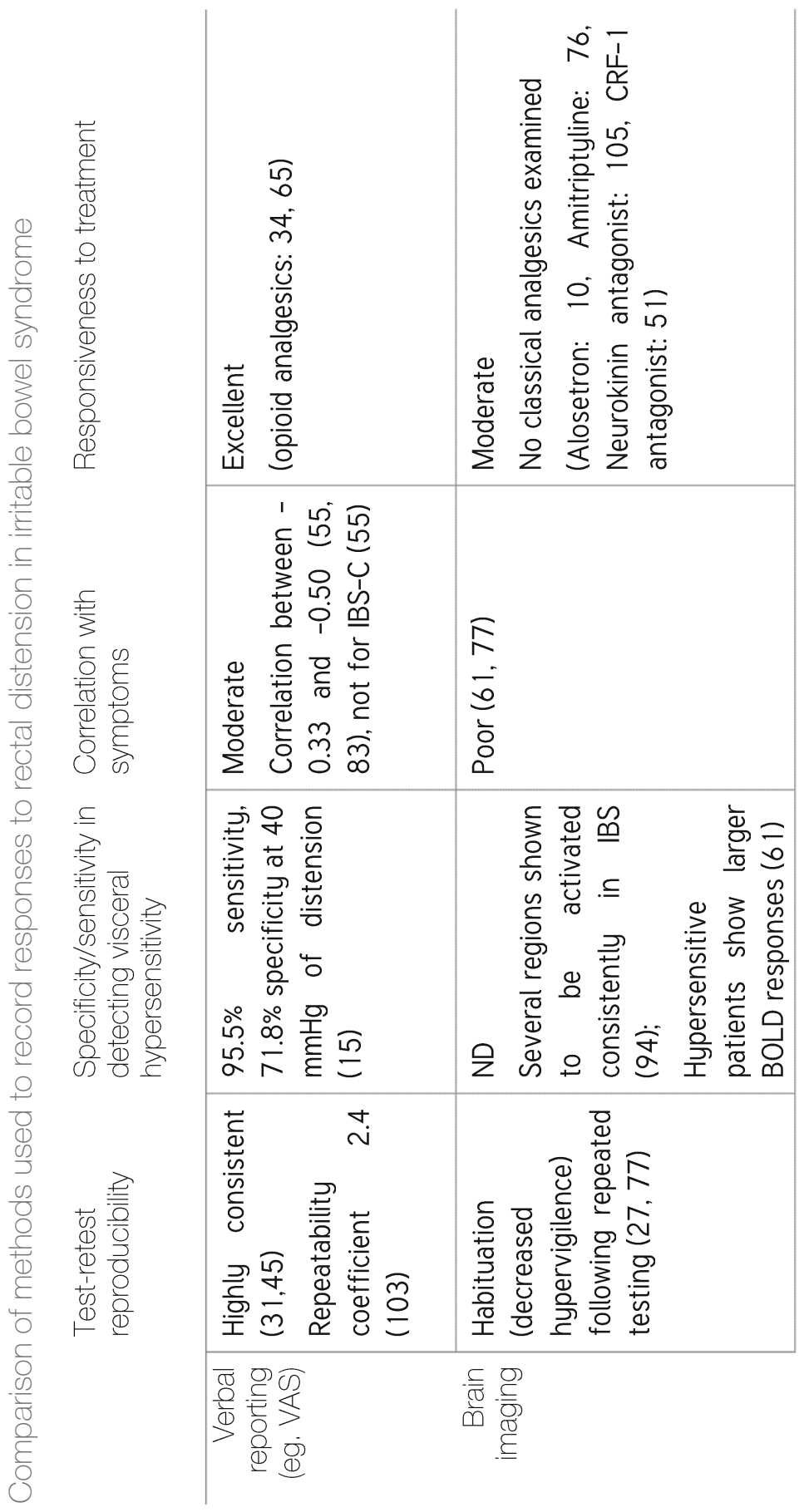




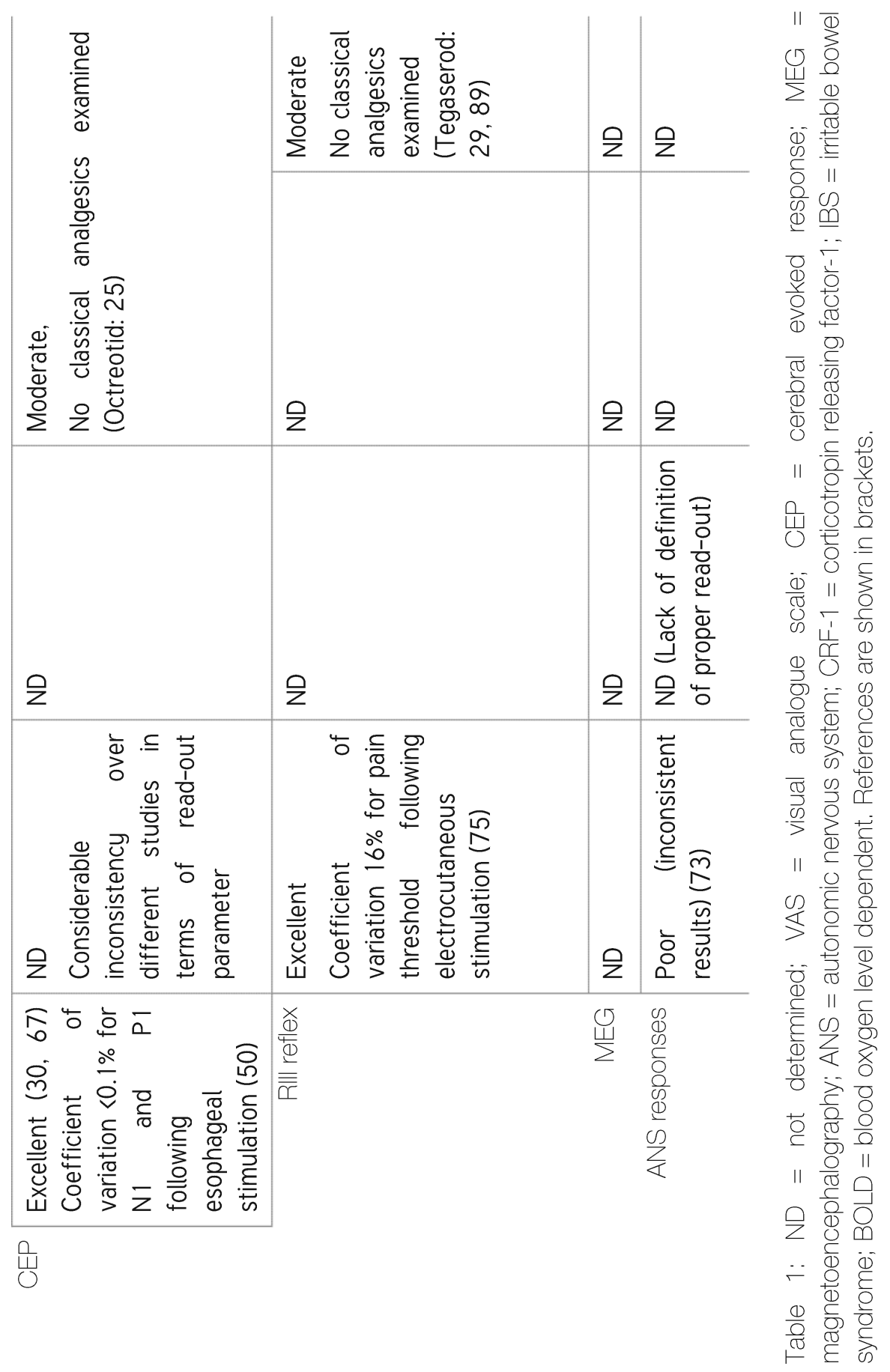


As for rectal distension and verbal reporting, almost 40 years have passed since its first introduction by Ritchie, allowing extensive characterization of the method. The use of brain imaging techniques in the study of visceral pain, on the other hand, is a relative novelty. Although these techniques represent an unparalleled opportunity to observe cortical processes during nociception, up to now, results of brain imaging studies in IBS have varied considerably or have been contradictory. This imposes a burden in establishing alterations in brain activity as an objective and reliable measure of visceral perception. In fact, these techniques potentially raise more questions than answers. Reaching a consensus about observed changes has proven to be difficult due to the variety of experimental paradigms (including subliminal, percept-related and stimulus related), analytic techniques, reporting practices and heterogeneous study populations (including patients with and without visceral hypersensitivity). Furthermore, it has become apparent that responses observed during brain imaging are also largely influenced by psychological factors. This questions the idea whether brain activation patterns can become superior to verbal reporting as a marker for visceral hypersensivity. Although both methods share the same shortcomings (influence by psychological factors, poor symptom correlation), verbal reporting seems to be more reproducible and relevant in detecting responsiveness to treatment (see Table 1). Certainly, brain imaging will not answer all of the questions but used in combination with other physiological, neurophysiological, and psychological techniques, it will surely remain an important tool in the gastrointestinal researchers armory with respect to the systematic evaluation of the mechanisms in pain processing.

Despite the growing body of literature, a number of challenges in the field still remain. First, chronic pain is not linearly related to peripheral sensory input. Current techniques involve stimulation of the distal colon with certain stimuli (that be mechanical, thermal, electrical or chemical) to excite peripheral afferents and evoke a sensory response, which is assessed by recording changes in brain activity or scoring subjective perception. These approaches are compounded by strong emotional and neurobehavioral components of pain, even though stimulation protocols can be optimized to minimalize effects of 
hypervigilance. The fact that activation of the same gastrointestinal pain neuromatrix can occur in the absence of a stimulus means that it is imperative that such factors are taken into consideration when assessing visceral perception in IBS. An anxious claustrophobic patient placed inside a noisy magnetic resonance scanner, with the anticipation of a large balloon to be painfully distended, may be forgiven for sustaining their attention throughout the investigation. ${ }^{116}$ Therefore, the subjective endpoint of pain or discomfort when assessing visceral perception remain inadequate because of susceptibility to response bias related to the psychological factors, with a higher tendency of patients with functional gut disorders to report pain.

Much of this difficulty lies in the complex nature and intrinsic heterogeneity of the condition. Brain imaging studies have also showed extensive activation in the arousal network, even in the absence of the actual stimulus. Therefore, it can be assumed that unless specific study paradigms are designed to differentiate brain responses to anticipation from the actual stimulus, brain responses during distension would also largely be influenced by the anticipation response. This distinction is especially difficult as these processes are functionally distributed in the same neural circuits.

Second, delineation between different mechanisms involved in pain generation in individual patients is necessary in order to determine therapeutic strategies. Following initial excitement of the clear demonstration of altered activation patterns in brain imaging studies, it remains unclear whether abnormal central activation patterns seen in brain imaging studies are related to a) enhanced selective attentional processes (hypervigilance); b) compromised endogenous pain inhibition system or facilitation of central pain processing at spinal or supraspinal level or c) normal processing of pathological visceral input from the periphery due to peripheral sensitization. Recent advances in electrophysiological recordings have provided very intriguing results with respect to the identification of these different mechanisms in this heterogeneous population of patients, however these results still await validation and characterization. Future research will have to establish whether these neurophysiological responses can 
become legitimate and objective measures for visceral hypersensivity in IBS. As pain is a conscious feeling, the ultimate goal in pain-imaging will be to follow the pain stimulus throughout the neuraxis, and to identify the locations of altered nociceptive processing, which is a prerequisite for adequate therapeutic correction.

Third, a further challenge in visceral sensitivity testing is that currently used methods only poorly correlate with symptoms. Poor correlation has been found between perception thresholds to rectal stimuli and symptom severity, 52,63 as well as between changes in brain responses to rectal stimulation during repeated stimulation and associated changes in symptom severity. ${ }^{17}$ This implies that changes observed during sensory testing using administration of an acute stimulus might not even relate to the generation of chronic pain symptoms. Therefore, a further provision for exploring novel therapeutic entities will be providing proof that symptoms are attributed to altered sensitivities, which can be tested clinically and that therapies aimed at the correction of hypersensitivity result in clinical benefit. ${ }^{118}$ As visceral hypersensitivity remains a paramount clinical phenomenon observed in IBS, methods to assess visceral perception, including the barostat approach and novel imaging techniques, have prompted further interest to unravel mechanisms of altered pain processing in IBS. Addressing the methodological shortcomings will potentially result in the identification of a robust objective clinical marker. Multimodal approaches, using the combination of verbal reporting as well as hemodynamic and electrophysiological responses for instance, have the potential to compensate for the shortcomings of the individuals methods. This will allow us to further elucidate visceral pain mechanisms and indeed open new horizons in the treatment of chronic pain in IBS. 
Comparison of brain imaging and neurophysiological techniques.

\begin{tabular}{|c|c|c|}
\hline $\begin{array}{l}\text { Temporal } \\
\text { resolution }\end{array}$ & $\begin{array}{l}\text { Spatial } \\
\text { resolution }\end{array}$ & Advantage \\
\hline
\end{tabular}

\begin{tabular}{|c|c|c|c|c|}
\hline fMRI & $1-3 \mathrm{sec}$ & $2-5 \mathrm{~mm}$ & $\begin{array}{ll}\text { Safe } & \text { (no } \\
\text { radiation) }\end{array}$ & $\begin{array}{l}\text { Artifacts produced by } \\
\text { pulsation, interference of } \\
\text { endogenous brain activity }\end{array}$ \\
\hline PET & $40-60 \mathrm{sec}$ & $2-5 \mathrm{~mm}$ & $\begin{array}{l}\text { Allows receptor } \\
\text { studies }\end{array}$ & $\begin{array}{l}\text { Radiation, costs, limited } \\
\text { information on anatomy, } \\
\text { interference of endogenous } \\
\text { brain activity }\end{array}$ \\
\hline P & $100 \mathrm{~ms}$ & poor & $\begin{array}{l}\text { Safe and } \\
\text { inexpensive }\end{array}$ & $\begin{array}{l}\text { Time-consuming, limited } \\
\text { availability, lacking } \\
\text { validation, Considerable } \\
\text { signal distortion }\end{array}$ \\
\hline MEG & $100 \mathrm{~ms}$ & $2-5 \mathrm{~mm}$ & $\begin{array}{l}\text { Allows dynamic } \\
\text { interpretation of } \\
\text { brain area } \\
\text { activation after } \\
\text { visceral } \\
\text { stimulation }\end{array}$ & $\begin{array}{l}\text { Limited availability, very } \\
\text { limited knowledge on } \\
\text { visceral pain testing, poor } \\
\text { signals from deeper brain } \\
\text { regions }\end{array}$ \\
\hline $\begin{array}{r}\text { Rill } \\
\text { reflex }\end{array}$ & $85-120 \mathrm{~ms}$ & poor & $\begin{array}{l}\text { Safe and } \\
\text { inexpensive }\end{array}$ & Lacking validation \\
\hline
\end{tabular}

Table 2: $\mathrm{fMRI}=$ functional magnetic resonance imaging; $\mathrm{PET}=$ positron emission tomography; CEP = cerebral evoked potential; $\mathrm{MEG}$ = magnetoencephalography. 


\subsection{REFERENCES}

1. Drossman DA, Camilleri M, Mayer EA, Whitehead WE. AGA technical review on irritable bowel syndrome. Gastroenterology 2002;123:2108-31.

2. Mertz H, Naliboff B, Munakata J, Niazi N, Mayer EA. Altered rectal perception is a biological marker of patients with irritable bowel syndrome. Gastroenterology 1995; 109:40-52.

3. Keszthelyi D, Troost FJ, Simren M, Ludidi S, Kruimel JW, Conchillo JM, Masclee AA. Revisiting concepts of visceral nociception in irritable bowel syndrome. Eur J Pain 2012.

4. Azpiroz F, Bouin M, Camilleri M, Mayer EA, Poitras P, Serra J, Spiller RC. Mechanisms of hypersensitivity in IBS and functional disorders. Neurogastroenterol Motil 2007; 19:62-88.

5. Blackshaw LA, Gebhart GF. The pharmacology of gastrointestinal nociceptive pathways. Curr Opin Pharmacol 2002;2:642-9.

6. Andrews PL, Sanger GJ. Abdominal vagal afferent neurones: an important target for the treatment of gastrointestinal dysfunction. Curr Opin Pharmacol 2002;2:650-6.

7. Brierley SM, Jones RC, 3rd, Gebhart GF, Blackshaw LA. Splanchnic and pelvic mechanosensory afferents signal different qualities of colonic stimuli in mice. Gastroenterology 2004;127:166-78.

8. Olsson C, Chen BN, Jones S, Chataway TK, Costa M, Brookes SJ. Comparison of extrinsic efferent innervation of guinea pig distal colon and rectum. J Comp Neurol 2006;496:787-801.

9. Feng B, Brumovsky PR, Gebhart GF. Differential roles of stretch-sensitive pelvic nerve afferents innervating mouse distal colon and rectum. Am J Physiol Gastrointest Liver Physiol 20 10;298:G402-9.

10. Kyloh M, Nicholas S, Zagorodnyuk VP, Brookes SJ, Spencer NJ. Identification of the visceral pain pathway activated by noxious colorectal distension in mice. Front Neurosci 2011;5:16.

11. Sengupta JN. Visceral pain: the neurophysiological mechanism. Handb Exp Pharmacol 2009:31-74.

12. Hughes PA, Brierley SM, Martin CM, Brookes SJ, Linden DR, Blackshaw LA. Postinflammatory colonic afferent sensitisation: different subtypes, different pathways and different time courses. Gut 2009;58:1333-41.

13. Feng B, Gebhart GF. Characterization of silent afferents in the pelvic and splanchnic innervations of the mouse colorectum. Am J Physiol Gastrointest Liver Physiol 2011;300:G 170-80.

14. Brierley SM, Carter R, Jones W, 3rd, Xu L, Robinson DR, Hicks GA, Gebhart GF, Blackshaw LA. Differential chemosensory function and receptor expression of splanchnic and pelvic colonic afferents in mice. J Physiol 2005;567:267-81.

15. Brierley SM, Jones RC, 3rd, Xu L, Gebhart GF, Blackshaw LA. Activation of splanchnic and pelvic colonic afferents by bradykinin in mice. Neurogastroenterol Motil 2005; 17:854-62.

16. Anand P, Aziz Q, Willert R, van Oudenhove L. Peripheral and central mechanisms of visceral sensitization in man. Neurogastroenterol Motil 2007;19:29-46. 
17. Feng B, La JH, Schwartz ES, Tanaka T, McMurray TP, Gebhart GF. Long-term sensitization of mechanosensitive and -insensitive afferents in mice with persistent colorectal hypersensitivity. Am $J$ Physiol Gastrointest Liver Physiol 2012;302:G676-83.

18. Al-Chaer ED, Traub RJ. Biological basis of visceral pain: recent developments. Pain 2002;96:221-5.

19. Sugiura Y, Terui N, Hosoya Y, Tonosaki Y, Nishiyama K, Honda T. Quantitative analysis of central terminal projections of visceral and somatic unmyelinated (C) primary afferent fibers in the guinea pig. J Comp Neurol 1993;332:315-25.

20. Ammons WS, Girardot MN, Foreman RD. T2-T5 spinothalamic neurons projecting to medial thalamus with viscerosomatic input. J Neurophysiol 1985;54:73-89.

21. Tillisch K, Mayer EA, Labus JS. Quantitative meta-analysis identifies brain regions activated during rectal distension in irritable bowel syndrome. Gastroenterology 2011;140:91-100.

22. Casey KL. Reticular formation and pain: toward a unifying concept. Res Publ Assoc Res Nerv Ment Dis 1980;58:93-105.

23. Willis WD, Westlund KN. Neuroanatomy of the pain system and of the pathways that modulate pain. J Clin Neurophysiol 1997;14:2-31.

24. White JC. Early studies have demonstrated that sensation of visceral distension was retained following extensive anterolateral chordotomy Research Publications Association for Research in Nervous and Mental Disease 1943;23:373-390.

25. Willis WD, Al-Chaer ED, Quast MJ, Westlund KN. A visceral pain pathway in the dorsal column of the spinal cord. Proc Natl Acad Sci U S A 1999;96:7675-9.

26. Chung JM, Lee KH, Surmeier DJ, Sorkin LS, Kim J, Willis WD. Response characteristics of neurons in the ventral posterior lateral nucleus of the monkey thalamus. J Neurophysiol 1986;56:370-90.

27. Price DD. Central neural mechanisms that interrelate sensory and affective dimensions of pain. Mol Interv 2002;2:392-403, 339.

28. Derbyshire SW. A systematic review of neuroimaging data during visceral stimulation. Am J Gastroenterol 2003;98:12-20.

29. Sheehan J, Gaman A, Vangel M, Kuo B. Pooled analysis of brain activity in irritable bowel syndrome and controls during rectal balloon distension. Neurogastroenterol Motil 2011;23:336-46, e158.

30. Pessoa L. On the relationship between emotion and cognition. Nat Rev Neurosci 2008;9:148-58.

31. Wager TD, Rilling JK, Smith EE, Sokolik A, Casey KL, Davidson RJ, Kosslyn SM, Rose RM, Cohen JD. Placebo-induced changes in FMRI in the anticipation and experience of pain. Science 2004;303:1162-7.

32. Bingel U, Lorenz J, Schoell E, Weiller C, Buchel C. Mechanisms of placebo analgesia: rACC recruitment of a subcortical antinociceptive network. Pain 2006;120:8-15.

33. Larsson MB, Tillisch K, Craig AD, Engstrom M, Labus J, Naliboff B, Lundberg P, Strom M, Mayer EA, Walter SA. Brain responses to visceral stimuli reflect visceral sensitivity thresholds in patients with irritable bowel syndrome. Gastroenterology 2012;142:463-472 e3.

34. Berman SM, Naliboff BD, Suyenobu B, Labus JS, Stains J, Ohning G, Kilpatrick L, 
Bueller JA, Ruby K, Jarcho J, Mayer EA. Reduced brainstem inhibition during anticipated pelvic visceral pain correlates with enhanced brain response to the visceral stimulus in women with irritable bowel syndrome. J Neurosci 2008;28:34959.

35. Mayer EA. Spinal and supraspinal modulation of visceral sensation. Gut 2000;47 Suppl 4:iv69-72; discussion iv76.

36. Mason P. Chapter 15 Descending pain modulation as a component of homeostasis. Handb Clin Neurol 2006;81:211-8.

37. Basbaum Al, Fields HL. Endogenous pain control systems: brainstem spinal pathways and endorphin circuitry. Annu Rev Neurosci 1984;7:309-38.

38. Sharma A, Aziz Q. Mechanisms of visceral sensitization in humans. In: Pasricha PJ, Willis WD, Gebhart GF, eds. Chronic Abdominal and Visceral Pain. New York, NY: Informa Healthcare USA, 2007.

39. Le Bars D, Dickenson AH, Besson JM. Diffuse noxious inhibitory controls (DNIC). I. Effects on dorsal horn convergent neurones in the rat. Pain 1979;6:283-304.

40. De Broucker T, Cesaro P, Willer JC, Le Bars D. Diffuse noxious inhibitory controls in man. Involvement of the spinoreticular tract. Brain 1990;113 ( Pt 4):1223-34.

41. Wilder-Smith $\mathrm{CH}$. The balancing act: endogenous modulation of pain in functional gastrointestinal disorders. Gut 2011;60:1589-99.

42. Gebhart GF. Descending modulation of pain. Neurosci Biobehav Rev 2004;27:72937.

43. Gold M. Overview of pain and sensitization. In: Pasricha PJ, Willis WD, Gebhart GF, eds. Chronic Abdominal and Visceral Pain. New York, NY: Informa Healthcare USA, 2007.

44. Bouin M, Plourde V, Boivin M, Riberdy M, Lupien F, Laganiere M, Verrier P, Poitras P. Rectal distention testing in patients with irritable bowel syndrome: sensitivity, specificity, and predictive values of pain sensory thresholds. Gastroenterology 2002; 122:1771-7.

45. Delvaux M. Do we need to perform rectal distention tests to diagnose IBS in clinical practice? Gastroenterology 2002;122:2075-8.

46. Piche $M$, Arsenault $M$, Poitras $P$, Rainville $P$, Bouin $M$. Widespread hypersensitivity is related to altered pain inhibition processes in irritable bowel syndrome. Pain 2010;148:49-58.

47. Lembo T, Munakata J, Naliboff B, Fullerton S, Mayer EA. Sigmoid afferent mechanisms in patients with irritable bowel syndrome. Dig Dis Sci 1997;42:111220.

48. Drewes AM, Petersen P, Rossel P, Gao C, Hansen JB, Arendt-Nielsen L. Sensitivity and distensibility of the rectum and sigmoid colon in patients with irritable bowel syndrome. Scand J Gastroenterol 2001;36:827-32.

49. van der Schaar PJ, Lamers CB, Masclee AA. The role of the barostat in human research and clinical practice. Scand J Gastroenterol Suppl 1999;230:52-63.

50. Lembo T, Munakata J, Mertz H, Niazi N, Kodner A, Nikas V, Mayer EA. Evidence for the hypersensitivity of lumbar splanchnic afferents in irritable bowel syndrome. Gastroenterology 1994;107:1686-96.

51. Mayer EA, Bradesi S, Chang L, Spiegel BM, Bueller JA, Naliboff BD. Functional GI 
disorders: from animal models to drug development. Gut 2008;57:384-404.

52. Posserud I, Syrous A, Lindstrom L, Tack J, Abrahamsson H, Simren M. Altered rectal perception in irritable bowel syndrome is associated with symptom severity. Gastroenterology 2007;133:1113-23.

53. Price DD, Robinson ME, Verne GN. Measuring pain and hyperalgesia in persistent pain conditions with a special emphasis on irritable bowel syndrome. In: Pasricha PJ, Willis WD, Gebhart GF, eds. Chronic Abdominal and Visceral Pain. New York, NY: Informa Healthcare USA, 2007.

54. Price DD, Bush FM, Long S, Harkins SW. A comparison of pain measurement characteristics of mechanical visual analogue and simple numerical rating scales. Pain 1994;56:217-26.

55. Kingham JG, Dawson AM. Origin of chronic right upper quadrant pain. Gut 1985;26:783-8.

56. Spetalen S, Jacobsen MB, Vatn MH, Blomhoff S, Sandvik L. Visceral sensitivity in irritable bowel syndrome and healthy volunteers: reproducibility of the rectal barostat. Dig Dis Sci 2004;49:1259-64.

57. Hammer HF, Phillips SF, Camilleri M, Hanson RB. Rectal tone, distensibility, and perception: reproducibility and response to different distensions. Am J Physiol 1998;274:G584-90.

58. Harris ML, Hobson AR, Hamdy S, Thompson DG, Akkermans LM, Aziz Q. Neurophysiological evaluation of healthy human anorectal sensation. Am J Physiol Gastrointest Liver Physiol 2006;291:G950-8.

59. Cremonini F, Houghton LA, Camilleri M, Ferber I, Fell C, Cox V, Castillo EJ, Alpers DH, Dewit OE, Gray E, Lea R, Zinsmeister AR, Whorwell PJ. Barostat testing of rectal sensation and compliance in humans: comparison of results across two centres and overall reproducibility. Neurogastroenterol Motil 2005;17:810-20.

60. Lembo T, Naliboff BD, Matin K, Munakata J, Parker RA, Gracely RH, Mayer EA. Irritable bowel syndrome patients show altered sensitivity to exogenous opioids. Pain 2000;87:137-47.

61. Delvaux M, Louvel D, Lagier E, Scherrer B, Abitbol JL, Frexinos J. The kappa agonist fedotozine relieves hypersensitivity to colonic distention in patients with irritable bowel syndrome. Gastroenterology 1999;116:38-45.

62. Bradette M, Delvaux M, Staumont G, Fioramonti J, Bueno L, Frexinos J. Octreotide increases thresholds of colonic visceral perception in IBS patients without modifying muscle tone. Dig Dis Sci 1994;39:1171-8.

63. van der Veek PP, Van Rood YR, Masclee AA. Symptom severity but not psychopathology predicts visceral hypersensitivity in irritable bowel syndrome. Clin Gastroenterol Hepatol 2008;6:321-8.

64. Kuiken SD, Lindeboom R, Tytgat GN, Boeckxstaens GE. Relationship between symptoms and hypersensitivity to rectal distension in patients with irritable bowel syndrome. Aliment Pharmacol Ther 2005;22:157-64.

65. Kanazawa M, Palsson OS, Thiwan SI, Turner MJ, van Tilburg MA, Gangarosa LM, Chitkara DK, Fukudo S, Drossman DA, Whitehead WE. Contributions of pain sensitivity and colonic motility to IBS symptom severity and predominant bowel habits. Am J Gastroenterol 2008;103:2550-61. 
66. Simren M, Mansson A, Langkilde AM, Svedlund J, Abrahamsson H, Bengtsson U, Bjornsson ES. Food-related gastrointestinal symptoms in the irritable bowel syndrome. Digestion 200 1;63:108-15.

67. Ragnarsson $G$, Bodemar $G$. Pain is temporally related to eating but not to defaecation in the irritable bowel syndrome (IBS). Patients' description of diarrhea, constipation and symptom variation during a prospective 6-week study. Eur J Gastroenterol Hepatol 1998; 10:415-21.

68. Simren M, Abrahamsson H, Bjornsson ES. An exaggerated sensory component of the gastrocolonic response in patients with irritable bowel syndrome. Gut 2001;48:207.

69. Caldarella MP, Milano A, Laterza F, Sacco F, Balatsinou C, Lapenna D, Pierdomenico SD, Cuccurullo F, Neri M. Visceral sensitivity and symptoms in patients with constipation- or diarrhea-predominant irritable bowel syndrome (IBS): effect of a low-fat intraduodenal infusion. Am J Gastroenterol 2005;100:383-9.

70. van der Veek PP, Steenvoorden M, Steens J, van der Schaar PJ, Brussee J, Masclee AA. Recto-colonic reflex is impaired in patients with irritable bowel syndrome. Neurogastroenterol Motil 2007;19:653-9.

71. Simren M, Agerforz P, Bjornsson ES, Abrahamsson H. Nutrient-dependent enhancement of rectal sensitivity in irritable bowel syndrome (IBS). Neurogastroenterol Motil 2007;19:20-9.

72. Ludidi S, Conchillo JM, Keszthelyi D, Koning CJ, Vanhoutvin SA, Lindsey PJ, Leufkens AM, Kruimel JW, Jonkers DM, Masclee AA. Does meal ingestion enhance sensitivity of visceroperception assessment in irritable bowel syndrome? Neurogastroenterol Motil 2012;24:47-53, e3.

73. Sabate JM, Gorbatchef C, Flourie B, Jian R, Coffin B. Cholecystokinin octapeptide increases rectal sensitivity to pain in healthy subjects. Neurogastroenterol Motil 2002; 14:689-95.

74. Bearcroft CP, Perrett D, Farthing MJ. Postprandial plasma 5-hydroxytryptamine in diarrhoea predominant irritable bowel syndrome: a pilot study. Gut 1998;42:42-6.

75. Simren M, Simms L, D'Souza D, Abrahamsson H, Bjornsson ES. Lipid-induced colonic hypersensitivity in irritable bowel syndrome: the role of 5-HT3 receptors. Aliment Pharmacol Ther 2003; 17:279-87.

76. Accarino AM, Azpiroz F, Malagelada JR. Symptomatic responses to stimulation of sensory pathways in the jejunum. Am J Physiol 1992;263:G673-7.

77. Rossel P, Drewes AM, Petersen P, Nielsen J, Arendt-Nielsen L. Pain produced by electric stimulation of the rectum in patients with irritable bowel syndrome: further evidence of visceral hyperalgesia. Scand J Gastroenterol 1999;34:1001-6.

78. Li Y, Wang Y, Zuo X, Guo Y, Zhang H, Lu X, Li J, Desmond PV. Visceral perception thresholds after rectal thermal and pressure stimuli in irritable bowel syndrome patients. J Gastroenterol Hepatol 2004;19:187-91.

79. Lynn PA, Blackshaw LA. In vitro recordings of afferent fibres with receptive fields in the serosa, muscle and mucosa of rat colon. J Physiol 1999;518 ( Pt 1):271-82.

80. Fuhrer M, Vogelsang H, Hammer J. A placebo-controlled trial of an oral capsaicin load in patients with functional dyspepsia. Neurogastroenterol Motil 2011;23:918e397. 
81. Ness TJ, Gebhart GF. Visceral pain: a review of experimental studies. Pain 1990;41:167-234.

82. Turner R. Magnetic resonance imaging of brain function. Am J Physiol Imaging 1992;7:136-45.

83. Heiss WD, Herholz K. Brain receptor imaging. J Nucl Med 2006;47:302-12.

84. Coen SJ, Gregory LJ, Yaguez L, Amaro E, Jr., Brammer M, Williams SC, Aziz Q. Reproducibility of human brain activity evoked by esophageal stimulation using functional magnetic resonance imaging. Am J Physiol Gastrointest Liver Physiol 2007:293:G 188-97.

85. Naliboff BD, Berman S, Suyenobu B, Labus JS, Chang L, Stains J, Mandelkern MA, Mayer EA. Longitudinal change in perceptual and brain activation response to visceral stimuli in irritable bowel syndrome patients. Gastroenterology 2006;131:352-65.

86. Morgan V, Pickens D, Gautam S, Kessler R, Mertz H. Amitriptyline reduces rectal pain related activation of the anterior cingulate cortex in patients with irritable bowel syndrome. Gut 2005;54:601-7.

87. Berman SM, Chang L, Suyenobu B, Derbyshire SW, Stains J, Fitzgerald L, Mandelkern M, Hamm L, Vogt B, Naliboff BD, Mayer EA. Condition-specific deactivation of brain regions by 5-HT3 receptor antagonist Alosetron. Gastroenterology 2002;123:96977.

88. Lackner JM, Lou Coad M, Mertz HR, Wack DS, Katz LA, Krasner SS, Firth R, Mahl TC, Lockwood AH. Cognitive therapy for irritable bowel syndrome is associated with reduced limbic activity, GI symptoms, and anxiety. Behav Res Ther 2006;44:621-38.

89. Tillisch K, Labus J, Nam B, Bueller J, Smith S, Suyenobu B, Siffert J, McKelvy J, Naliboff B, Mayer E. Neurokinin-1-receptor antagonism decreases anxiety and emotional arousal circuit response to noxious visceral distension in women with irritable bowel syndrome: a pilot study. Aliment Pharmacol Ther 2012;35:360-7.

90. Hubbard CS, Labus JS, Bueller J, Stains J, Suyenobu B, Dukes GE, Kelleher DL, Tillisch K, Naliboff BD, Mayer EA. Corticotropin-releasing factor receptor 1 antagonist alters regional activation and effective connectivity in an emotionalarousal circuit during expectation of abdominal pain. J Neurosci 2011;31:12491500 .

91. Hobson AR, Sarkar S, Furlong PL, Thompson DG, Aziz Q. A cortical evoked potential study of afferents mediating human esophageal sensation. Am J Physiol Gastrointest Liver Physiol 2000;279:G 139-47.

92. Hobson AR, Furlong PL, Sarkar S, Matthews PJ, Willert RP, Worthen SF, Unsworth BJ, Aziz Q. Neurophysiologic assessment of esophageal sensory processing in noncardiac chest pain. Gastroenterology 2006;130:80-8.

93. Arebi N, Bullas DC, Dukes GE, Gurmany S, Hicks KJ, Kamm MA, Hobson AR. Distinct neurophysiological profiles in irritable bowel syndrome. Am J Physiol Gastrointest Liver Physiol 2011;300:G 1086-93.

94. Zuo XL, Li YQ, Huang KM, Kuang RG, Lv GP, Lu XF, Li JM, Desmond PV. Alterations in cerebral potentials evoked by rectal distention and drinking ice water in patients with irritable bowel syndrome. J Gastroenterol Hepatol 2006;21:1844-9.

95. Sinhamahapatra P, Saha SP, Chowdhury A, Chakrabarti SK, Ghosh A, Maiti B. Visceral 
afferent hypersensitivity in irritable bowel syndrome--evaluation by cerebral evoked potential after rectal stimulation. Am J Gastroenterol 2001;96:2150-7.

96. Chan YK, Herkes GK, Badcock C, Evans PR, Bennett E, Kellow JE. Alterations in cerebral potentials evoked by rectal distension in irritable bowel syndrome. Am J Gastroenterol 2001;96:2413-7.

97. Collet L, Meunier P, Duclaux R, Chery-Croze S, Falipou P. Cerebral evoked potentials after endorectal mechanical stimulation in humans. Am J Physiol 1988;254:G47782.

98. Loening-Baucke V, Read NW, Yamada T. Cerebral evoked potentials after rectal stimulation. Electroencephalogr Clin Neurophysiol 1991;80:490-5.

99. Chey WD, Beydoun A, Roberts DJ, Hasler WL, Owyang C. Octreotide reduces perception of rectal electrical stimulation by spinal afferent pathway inhibition. Am J Physiol 1995;269:G821-6.

100. Bromm B, Scharein E, Vahle-Hinz C. Cortex areas involved in the processing of normal and altered pain. Prog Brain Res 2000;129:289-302.

101. Williamson SJ, Lu ZL, Karron D, Kaufman L. Advantages and limitations of magnetic source imaging. Brain Topogr 1991;4:169-80.

102. Smith JK, Humes DJ, Head KE, Bush D, White TP, Stevenson CM, Brookes MJ, Marciani L, Spiller RC, Gowland PA, Francis ST. fMRI and MEG analysis of visceral pain in healthy volunteers. Neurogastroenterol Motil 2011;23:648-e260.

103. Sherrington CS. Flexion-reflex of the limb, crossed extension-reflex, and reflex stepping and standing. J Physiol 1910;40:28-121.

104. Hugon M. Exteroceptive reflexes to stimulation of the sural nerve in normal man. In: Desmedt JE, ed. New Developments in Electromyo- graphy and Clinical Neurophysiology. Basel: Karger, 1973.

105. Micalos PS, Drinkwater EJ, Cannon J, Arendt-Nielsen L, Marino FE. Reliability of the nociceptive flexor reflex (RIII) threshold and association with Pain threshold. Eur $\mathrm{J}$ Appl Physiol 2009; 105:55-62.

106. Sandrini G, Serrao M, Rossi P, Romaniello A, Cruccu G, Willer JC. The lower limb flexion reflex in humans. Prog Neurobiol 2005;77:353-95.

107. Bouhassira D, Sabate JM, Coffin B, Le Bars D, Willer JC, Jian R. Effects of rectal distensions on nociceptive flexion reflexes in humans. Am $J$ Physiol 1998;275:G410-7.

108. Coffin B, Bouhassira D, Sabate JM, Barbe L, Jian R. Alteration of the spinal modulation of nociceptive processing in patients with irritable bowel syndrome. Gut 2004;53:1465-70.

109. Coffin B, Farmachidi JP, Rueegg P, Bastie A, Bouhassira D. Tegaserod, a 5-HT4 receptor partial agonist, decreases sensitivity to rectal distension in healthy subjects. Aliment Pharmacol Ther 2003;17:577-85.

110. Sabate JM, Bouhassira D, Poupardin C, Wagner A, Loria Y, Coffin B. Sensory signalling effects of tegaserod in patients with irritable bowel syndrome with constipation. Neurogastroenterol Motil 2008;20:134-41.

111. lovino P, Azpiroz F, Domingo E, Malagelada JR. The sympathetic nervous system modulates perception and reflex responses to gut distention in humans. Gastroenterology 1995; 108:680-6. 
112. Mazurak N, Seredyuk N, Sauer H, Teufel M, Enck P. Heart rate variability in the irritable bowel syndrome: a review of the literature. Neurogastroenterol Motil 2012;24:206-16.

113. van der Veek PP, Swenne CA, Vooren H, Schoneveld AL, Maestri R, Masclee AA. Viscerosensory-cardiovascular reflexes: altered baroreflex sensitivity in irritable bowel syndrome. Am J Physiol Regul Integr Comp Physiol 2005;289:R970-6.

114. Spaziani R, Bayati A, Redmond K, Bajaj H, Mazzadi S, Bienenstock J, Collins SM, Kamath MV. Vagal dysfunction in irritable bowel syndrome assessed by rectal distension and baroreceptor sensitivity. Neurogastroenterol Motil 2008;20:336-42.

115. Dorn SD, Palsson OS, Thiwan SI, Kanazawa M, Clark WC, van Tilburg MA, Drossman DA, Scarlett Y, Levy RL, Ringel Y, Crowell MD, Olden KW, Whitehead WE. Increased colonic pain sensitivity in irritable bowel syndrome is the result of an increased tendency to report pain rather than increased neurosensory sensitivity. Gut 2007;56:1202-9.

116. Hobson AR, Aziz Q. Brain imaging and functional gastrointestinal disorders: has it helped our understanding? Gut 2004;53:1198-206.

117. Naliboff BD, Mayer EA. Brain imaging in IBS: drawing the line between cognitive and non-cognitive processes. Gastroenterology 2006;130:267-70.

118. Camilleri M. Testing the sensitivity hypothesis in practice: tools and methods, assumptions and pitfalls. Gut 2002;51 Suppl 1:i34-40. 
Alterations in mucosal neuropeptides in patients with irritable bowel syndrome and ulcerative colitis in remission: a role in pain symptom generation?

D. Keszthelyi, FJ. Troost, DM. Jonkers, Zs. Helyes, HM. Hamer, S. Ludidi,

S. Vanhoutvin, K. Venema, J. Dekker, J. Szolcsányi, AA. Masclee

European Journal of Pain 2013 Oct;17(9):1299-306

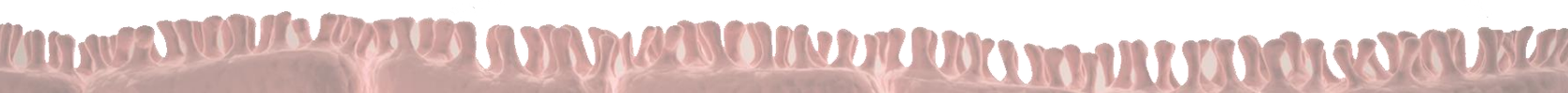




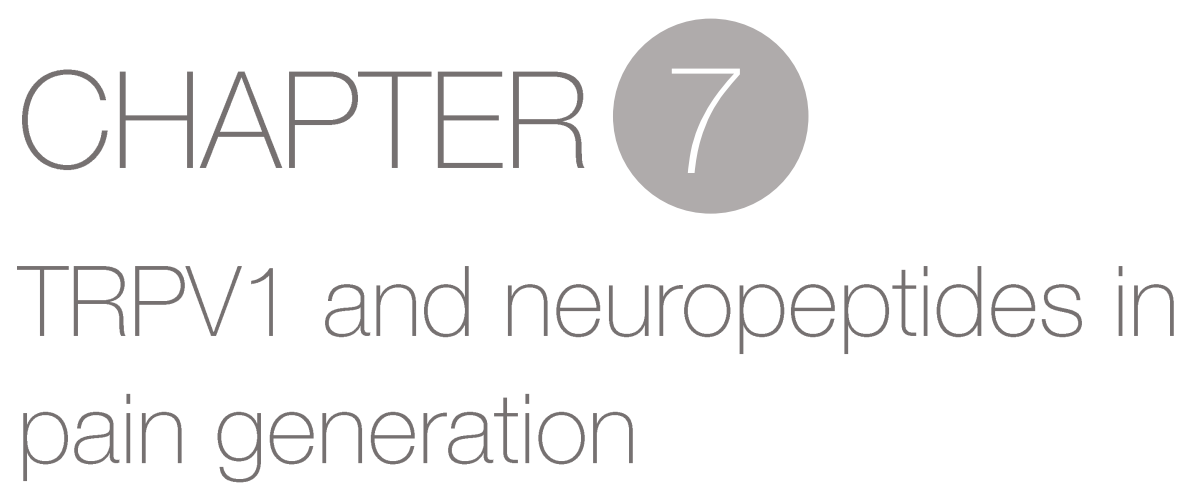

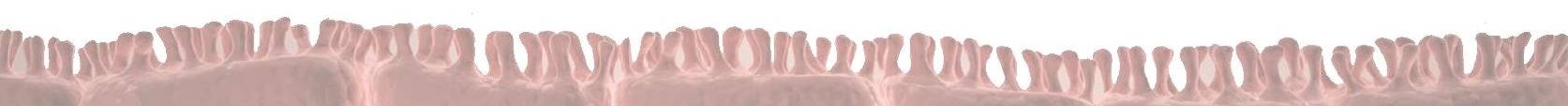




\section{ABSTRACT}

Background: Irritable bowel syndrome (IBS) is a functional gastrointestinal disorder characterized by chronic abdominal pain. The Transient Receptor Potential Vanilloid 1 (TRPV1) channel, which is involved in visceral pain signaling, has been shown to be upregulated in IBS. Activation of TRPV 1 leads to the release of neuropeptides, such as somatostatin and substance $P$ (SP). We hypothesized that increased pain perception in IBS could be explained by increased transcription in TRPV 1 and/or altered levels of neuropeptides. We therefore assessed the transcription of TRPV 1 and the mucosal concentration of somatostatin and SP in IBS in comparison to healthy volunteers and patients with ulcerative colitis (UC) in remission as disease controls, and to ascertain their relationship to pain symptoms.

Method: Sigmoid colonic mucosal samples were collected from 12 patients with IBS, 34 patients with UC in remission and 9 healthy volunteers, in which groups TRPV 1 mRNA levels were determined using $\mathrm{QPCR}$ and neuropeptide concentrations by radioimmunoassay. Pain symptom intensity was determined by questionnaires.

Results: Transcription of TRPV1 as well as the concentration of neuropeptides were significantly higher in IBS, but only the former correlated with pain symptom severity.

Conclusion: Increased transcription of TRPV 1 may provide a possible explanation for pain generation in IBS. While the neuropeptides SP and somatostatin were both found to be increased in IBS, these changes are not sufficient to explain pain generation. Pain generation in IBS is probably explained by a complex redundancy in the regulation of local nociceptive mechanisms, which remains subject of intensive investigation. 


\subsection{INTRODUCTION}

Irritable bowel syndrome (IBS) is a functional gastrointestinal disorder characterized by chronic abdominal pain and altered bowel habits. Although the pathophysiology of pain generation in IBS is still poorly understood, one of the possible mechanisms involved is an increase in input from mucosal afferents resulting in augmented pain perception. Recently, molecular findings have prompted interest in peripheral sensory physiology to explain increased pain perception in IBS.' Evidence suggests a role for the Transient Receptor Potential Vanilloid 1 (TRPV1) receptor, which functions as an important transducer of nociceptive signals. Increased expression of the TRPV 1 protein has been demonstrated in mucosal nerve fibres of IBS patients, which correlated with their pain symptom severity. ${ }^{2}$ Inflammatory processes have been postulated to underlie this increased expression. ${ }^{2}$ Interestingly, it appears that following inflammation, in contrast to normal conditions, not the TRPV 1 protein, but its mRNA is transported to the terminals. ${ }^{3}$ The increased biosynthesis of TRPV 1 at the nerve terminals is thought to be involved in an augmented sensitivity of TRPV $1,{ }^{4}$ potentially resulting in increased afferent discharge. In this respect, assuming that mucosal inflammation is a potential mechanistic factor in IBS, it is of important relevance to investigate potential alterations in mucosal mRNA levels in IBS.

To investigate the possible role for a preceding mucosal inflammation in inducing such alterations in pain processing, we also investigated mucosal samples from patients with ulcerative colitis (UC) in remission. One-third of patients with UC in remission demonstrate bowel symptoms compatible with IBS, in absence of signs of ongoing active inflammation.5, 6 Furthermore, TRPV 1 immunoreactivity has been shown to be elevated in mucosal samples of IBD patients with active diseases. ${ }^{7}$

TRPV 1 is expressed throughout the gastrointestinal tract by primary sensory neurons projecting to the mucosa. Activation of the receptor leads to depolarization resulting in pain sensation (afferent function). ${ }^{8}$ TRPV 1 activation also results in local release of sensory neuropeptides including calcitonin-gene related peptide (CGRP) and substance $P$ 
(SP), which in turn induce vasodilatation and plasma protein extravasation collectively called neurogenic inflammation (local efferent function). ${ }^{9}$ Activation of TRPV 1 also results in release of somatostatin. Endogenous somatostatin is thought to exert a tonic antinociceptive effect by acting prejunctionally on the sensory nerve terminals inhibiting the release and action of pro-nociceptive transmitters. ${ }^{10,11}$

Therefore, changes in TRPV 1 transcription and function might result in an imbalance between pro- and anti-nociceptive neuropeptides in the mucosa. Alterations in this precisely regulated balance in favor of the pro-nociceptive neuropeptides consequently may contribute to dysfunction in intestinal signaling and symptom generation in IBS and UC in remission. Therefore, the aim of our current study was to assess the transcription of TRPV 1, the mucosal concentration of the neuropeptides SP and somatostatin, and to ascertain their relationship to symptoms in IBS, UC patients in remission and healthy controls.

\subsection{METHODS}

\section{PATIENTS}

IBS patients, UC patients in clinical and endoscopic remission and healthy volunteers participated in this study. Patients were recruited via the gastroenterology outpatient clinic of the Maastricht University Medical Center, healthy volunteers via local advertisements. IBS patients were diagnosed according to the Rome III criteria. Patients with UC were diagnosed by clinical criteria (using the colitis activity index, CAI) and endoscopic examination. UC patients were in clinical remission for at least two years prior to inclusion, i.e. had no complaints of increased stool frequency or blood/mucus loss, and were included in the study if the Endoscopic Grading System ${ }^{12}$ score was lower than 5 at the time of mucosal sampling. All patients and healthy volunteers were between 18 and 65 years of age and consumed a stable Western diet. Exclusion criteria included pregnancy, lactation, changes in medication intake 2 weeks before and during the study, the use of medication altering intestinal physiology (NSAIDs, PPIs, H2 blockers, 
corticosteroids, SSRIs) 2 weeks before or during the study, the use of antibiotics 3 months before or during the study, other clinically significant systemic diseases and earlier radiotherapy or chemotherapy. Exclusion criteria for healthy volunteers also included any medical history of disease. All healthy volunteers and patients gave their written informed consent to participate. The collection of biopsy specimens obtained in this study had been approved by the Medical Ethics Committee of the Maastricht University Medical Centre (MUMC) and was conducted according to the revised version of the Declaration of Helsinki (October 2008, Seoul).

\section{STUDY PROCEDURES}

Sigmoid colonic mucosal samples were collected during standard sigmoidoscopy from all patients and healthy volunteers. Each sigmoidoscopy was performed in the morning after an overnight fasting period without prior bowel cleansing. During all sigmoidoscopies, four mucosal biopsies were taken from a standardized location of the sigmoid (approximately $20-25 \mathrm{~cm}$ from the anal verge at the location of the arteria iliaca communis). One biopsy specimen was prepared for a pathological examination using regular haematoxylin-eosin $(\mathrm{HE})$ staining, the other three specimens were frozen immediately and stored at $-80^{\circ} \mathrm{C}$ until analysis.

\section{SYMPTOM ASSESSMENT}

UC and IBS patients and healthy controls completed a questionnaire seven days prior to the endoscopy regarding bowel habits. The following symptoms were scored as absent (0), mild (1), moderate (2) or severe (3): nausea, abdominal cramps, abdominal pain, bloating, flatulence, blood in the feces, visible mucus in the feces and other, as described previously. ${ }^{13}$ For instance, if a patient experienced abdominal pain on a particular day, the patient gave this perception a score of 1 to 3 depending on the intensity of the pain perceived. It is important to note here that the questionnaire was developed for UC patients and not IBS patients. Nevertheless, in this study, only scores for pain symptoms have been analyzed. All scores were added up for each complaint over the seven-day period. The maximum score per item was therefore 21 . A composite pain score was 
calculated by adding up the daily scores for the items 'abdominal pain' and 'abdominal cramps'. Therefore, the composite score ranged from 0 to 42. In addition, the Colitis Activity Index (CAI) was determined in UC patients at time of the endoscopy.

\section{TRANSCRIPTION OF TRPV1}

Quantitative PCR was used to assess mRNA expression levels of TRPV 1, using 18S RNA and glyceraldehyde 3-phosphate dehydrogenase (GAPDH) as reference genes. Total RNA was extracted from the frozen biopsies using trizol (Invitrogen), followed by a cleaning step with the RNeasy Mini kit (Qiagen) combined with a DNase treatment using the RNasefree DNase Set (Qiagen) to efficiently remove DNA present. Quantification of the purified RNA was done using the NanoDrop method (Qiagen). CDNA was synthesized using the iScript cDNA Syntheses Kit (Bio-rad). PCR amplifications were run on the MylQ Real-Time PCR detection system. PCRs were performed with $5 \mu \mathrm{l}$ of CDNA template diluted to a concentration of $4 \mathrm{ng} / \mu \mathrm{l}, 12.5 \mu \mathrm{l}$ iQ Sybr Green supermix (Bio-rad), $1 \mu \mathrm{l}$ forward en reversed primers $(10 \mu \mathrm{M})$ and $5.5 \mu \mathrm{l}$ of sterile water. Cycling conditions were as follows: $3 \mathrm{~min}$ at $95^{\circ} \mathrm{C}$ for denaturation of cDNA followed by 40 amplification cycles of 10 seconds at $95^{\circ} \mathrm{C}$ and annealing for $45 \mathrm{~s}$ at $60^{\circ} \mathrm{C}$. The following primers were used:

> GAPDH forward: 5'-TGCACCACCAACTGCTTAGC-3'

> GAPDH reverse: 5'-GGCATGGACTGTGGTCATGAG-3'

> 18S rRNA forward: 5'-GTAACCCGTTGAACCCCATT-3'

> 18S rRNA reverse: 5'-CCATCCAATCGGTAGTAGCG-3'

> TRPV 1 forward: 5'-TCTCACCTACATCCTCCTGCTCAA-3'

> TRPV 1 reverse: 5'-TTGCTCTCCTGTGCGATCTTGT-3'

\section{CONCENTRATIONS OF SOMATOSTATIN AND SUBSTANCE P (SP)}

Mucosal concentrations of somatostatin and SP were determined using radioimmunoassay (RIA) as previously described in details.14, 15 In brief, samples were weighed and homogenized in $1 \mathrm{ml}$ ice-cold $20 \mathrm{mM}$ potassium-phosphate buffer (pH 7.4) with a manual potter homogenizer. The homogenates were centrifuged at 10,000 rpm at 
$4{ }^{\circ} \mathrm{C}$ for $10 \mathrm{~min}$ and then at $12,000 \mathrm{rpm}$ for another $10 \mathrm{~min}$, and $70 \mu \mathrm{l}$ samples of the supernatants were used for RIA measurements of SP and somatostatin. The detection limit of the assay was $2 \mathrm{fmol} / \mathrm{ml}$ in case of $\mathrm{SP}$ and $0.2 \mathrm{fmol} / \mathrm{ml}$ for somatostatin determination. Measurements of neuropeptide concentrations were limited to somatostatin and substance $P$ due to relatively large amounts of material necessary for RIA measurements.

\section{STATISTICAL ANALYSES}

Statistical analyses were performed using Graphpad Prism 4.0 (GraphPad Software Inc, LaJolla, CA) and SPSS 20 (SPSS Inc, Chicago, IL). Normality of the data was determined using Kolmogorov-Smirnoff test. Data normally distributed are expressed as mean $\pm \mathrm{SEM}$, and were analyzed using one-way ANOVA and Tukey's post-hoc test. Data not normally distributed are expressed as median and range, and were analyzed by Kruskall-Wallis and Dunn's post-hoc test. Correlations were determined according to Pearson and Spearmen for parametric and non-parametric data, respectively. PCR data are expressed as mean expression ratios \pm SEM, normalized for the housekeeping genes. Gender differences and relation to IBS subtypes were assessed by $x^{2}$ test.

\subsection{RESULTS \\ PATIENT CHARACTERISTICS}

Twelve patients with IBS (age $41 \pm 4$ years, 33\% male), 34 patients with UC in clinical and endoscopic remission (age $52 \pm 2$ years, $50 \%$ male) and the 9 healthy volunteers ( $35 \pm 4$, $33 \%$ male) were included. All participants were of Caucasian race. IBS subtypes consisted of $58 \%$ IBS-D (diarrhea-predominant), 33\% IBS-C (constipation-predominant) and $8 \%$ IBS$M$ (mixed subtype). All UC patients included have had the disease for at least 4 years prior to inclusion. $58 \%$ of the UC patients were on anti-inflammatory medication (mostly mesalazine, mercaptopurine or azathioprine). No association was found between age, gender, IBS subtype, use of anti-inflammatory drugs and any of the studied parameters such as neuropeptide concentrations, TRPV 1 transcription and composite pain score. 


\section{COMPOSITE PAIN SCORE}

IBS patients had a median composite score of 10.5 (range 7-30) for abdominal pain and/or cramping. All IBS patients complained of some degree of abdominal pain or cramping (see Figure 1).

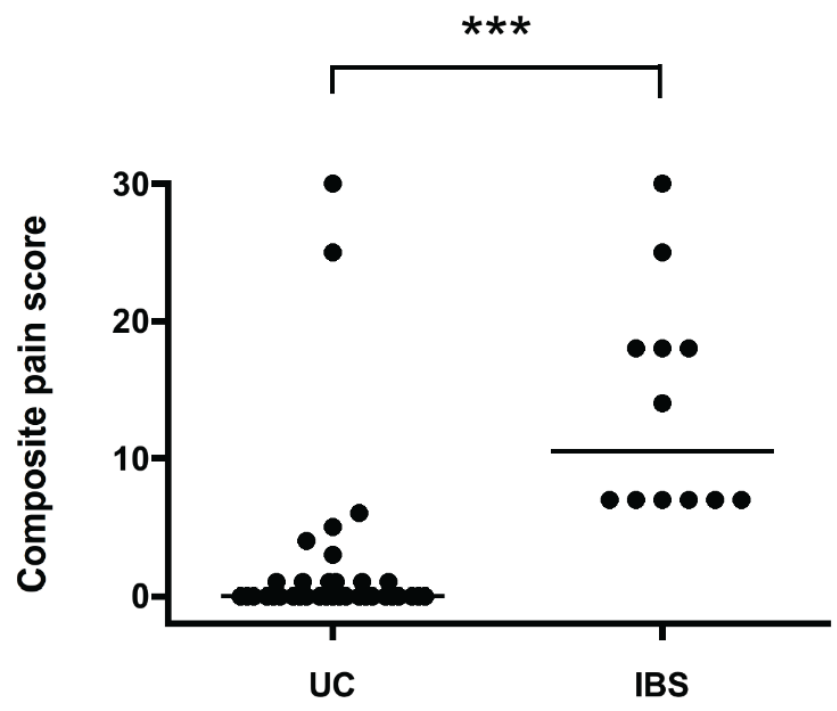

Figure 1: Composite pain score in patients with ulcerative colitis $(U C)$ in remission and irritable bowel syndrome (IBS), ${ }^{* \star *} \mathrm{p}<0.001$

UC patients had a median composite pain score of 0 (range $0-30$, see Figure 1). Of the UC patients, 11 (32\%) complained of abdominal symptoms (age $50 \pm 3$ yrs, 45\% male). These patients had a median composite pain score of 2 (range 1-30). UC patients with or without complaints did not differ significantly in terms of age or gender.

A median of 2 (range $0-10$ ) for CAI was observed in UC patients. No correlation was found between CAI score and any of the investigated markers (neuropeptides and TRPV 1 transcription).

None of the healthy controls complained of abdominal pain or cramping. Therefore, all 
healthy controls had a composite pain score of 0 .

Mucosal samples from all patients and healthy controls were also examined microscopically for mucosal inflammation. None of the UC patients showed signs of active inflammation confirming that their disease was in remission. Similarly, IBS patients showed no inflammatory changes and/or microscopic colitis.

\section{TRPV1 TRANSCRIPTION}

Significant differences were observed regarding the transcription of TRPV 1 between IBS patients, UC patients and healthy controls $(p=0.02)$. Post hoc analysis revealed a higher transcription in IBS patients compared to controls $(p=0.01)$ and to UC patients $(p=0.03)$ (see Figure 2). No significant differences were found in the transcription of TRPV 1 when comparing UC patients with or without complaints.

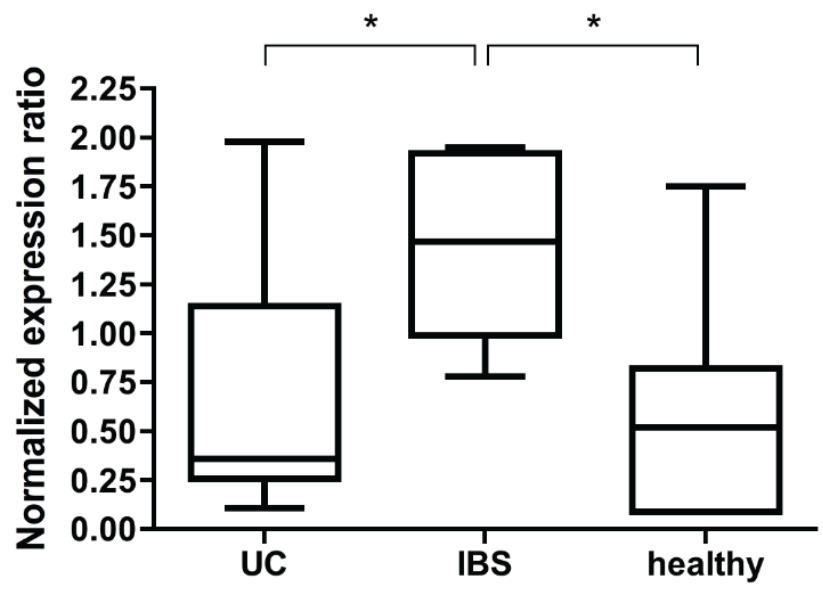

Figure 2: Normalized expression ratios of the Transient Receptor Potential Vanilloid 1 (TRPV1) receptor in patients with irritable bowel syndrome (BSS), ulcerative colitis (UC) in remission and healthy controls. ${ }^{*} \mathrm{p}<0.05$

In IBS patients, significant correlation was found between the composite score pain symptom severity and TRPV1 transcription $(r=0.76, p=0.04)$. In UC patients, as a group, 


\section{Chapter 7}

no correlation was found between symptom severity and TRPV1 transcription. However, when UC patients with pain complaints were analyzed separately, TRPV 1 transcription correlated significantly with the composite score for abdominal pain $(r=0.70, p=0.004)$.

\section{CONCENTRATIONS OF SOMATOSTATIN AND SUBSTANCE P}

The concentration of somatostatin was significantly higher in IBS patients compared to UC patients in remission and vs healthy controls (see Table 1 and Figure 3A). Mucosal somatostatin concentration was also higher in IBS when compared to UC patients without complaints, but not compared to UC patients with abdominal complaints. Mucosal levels of SP in IBS patients were significantly higher in IBS patients compared to UC patients, but not compared to healthy controls (Table 1; Figure 3B). Similarly to somatostatin, higher concentration of SP was found in IBS when compared to UC patients without complaints, but not compared to UC patients with abdominal complaints (Table 1). No significant differences in neuropeptide concentrations were observed when comparing UC patients with or without abdominal complaints.

A
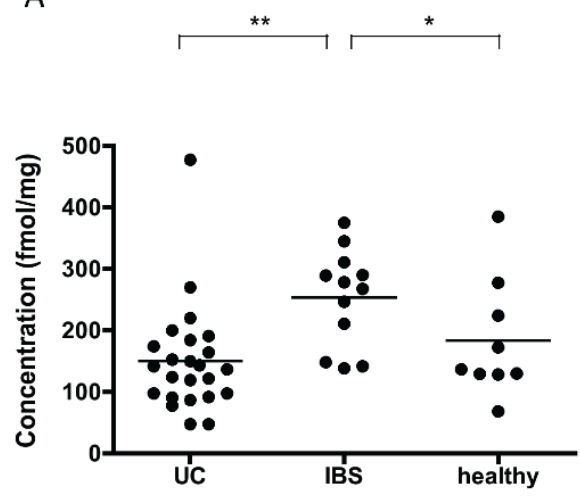

B
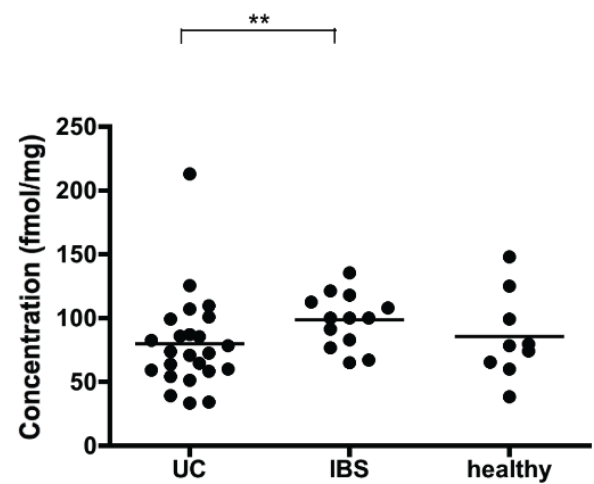

Figure 3: (A) Mucosal concentrations of somatostatin (fmol/mg tissue) in in patients with irritable bowel syndrome (IBS), ulcerative colitis $(U C)$ in remission and healthy controls. ${ }^{*} \mathrm{P}<0.05,{ }^{* *} \mathrm{P}<0.01$. (B) Mucosal concentrations of substance $P(\mathrm{SP}, \mathrm{fmol} / \mathrm{mg}$ tissue) in in patients with irritable bowel syndrome (IBS), ulcerative colitis (UC) in remission and healthy controls. ${ }^{* *} \mathrm{p}<0.01$. 


\begin{tabular}{r|lllll}
\multicolumn{2}{c}{$\begin{array}{l}\text { IBS patients } \\
N=12\end{array}$} & $\begin{array}{l}\text { UC } \\
\text { patients } \\
\text { (all) } \\
N=34\end{array}$ & $\begin{array}{l}\text { UC patients } \\
\text { with } \\
\text { complaints } \\
N=11\end{array}$ & $\begin{array}{l}\text { UC patients } \\
\text { without } \\
\text { complaints } \\
N=23\end{array}$ & $\begin{array}{l}\text { Healthy } \\
\text { controls } \\
N=9\end{array}$ \\
\hline $\begin{array}{r}\text { Somatostatin } \\
(\mathrm{fmol} / \mathrm{mg})\end{array}$ & $257 \pm 21^{\mathrm{a}, \mathrm{b}, \mathrm{c}}$ & $173 \pm 18$ & $213 \pm 46$ & $150 \pm 18$ & $173 \pm 31$ \\
$\mathrm{SP}$ & $95 \pm 6^{\mathrm{a}, \mathrm{b}}$ & $83 \pm 7$ & $85 \pm 19$ & $80 \pm 8$ & $88 \pm 11$ \\
$(\mathrm{fmol} / \mathrm{mg})$ & & & & &
\end{tabular}

Table 1: Neuropeptide concentrations in irritable bowel syndrome (IBS) patients, patients with UC (ulcerative colitis) in remission (also subdivided into patients with and without symptoms) and healthy controls. Data expressed as mean \pm SEM.

${ }^{a} \mathrm{p}<0.01$ IBS vs UC (all)

${ }^{b} \mathrm{p}<0.01$ IBS vs UC without complaints

${ }^{\circ} \mathrm{p}<0.05$ IBS vs healthy

Concentrations of SP and somatostatin correlated significantly when data from each group was pooled together $\left(r^{2}=0.68, p<0.0001\right)$. When analyzed per group, significant correlation between the neuropeptides SP and somatostatin was observed in UC patients $\left(r^{2}=0.76, p<0.0001\right)$ and healthy controls $\left(r^{2}=0.56, p=0.02\right)$, but not in IBS patients $\left(r^{2}=0.25\right.$, p>0.05). This lack of positive correlation in the IBS group was seen together with relatively higher concentrations of somatostatin compared to SP.

No significant correlation was found between pain symptom severity with any of the neuropeptides in either patient group, neither when subdividing UC patients into groups with or without abdominal pain complaints. Similarly, no significant correlation was found between TRPV 1 transcription and SP or somatostatin concentration, possibly due to the fact that TRPV 1 positive nerve fibres are not the sole source of mucosal neuropeptides. 


\subsection{DISCUSSION}

In this study, we found evidence of an increased transcription of the TRPV 1 receptor, as well as increased mucosal concentrations of the neuropeptides substance $P$ and somatostatin in IBS patients. Significant correlation was found between pain symptom severity and TRPV1 transcription, but not with mucosal neuropeptide concentrations.

The role of TRPV1 in visceral pain signaling has received increased attention in the past decade. To date, a significant increase in the number of TRPV1-immunoreactive fibres was demonstrated in mucosal samples of IBS patients and this has been shown to correlate with abdominal pain score. ${ }^{2}$ However, alterations in mucosal mRNA levels of TRPV 1 in IBS have previously not been reported. We demonstrate here an increased transcription of the TRPV 1 receptor, which, in line with the protein expression demonstrated by Akbar et al., was found to correlate with pain symptom severity.

The increased mRNA levels seen in the mucosa of IBS patients could therefore reflect an increased transport of mRNA to the mucosal nerve terminals. It has been described previously that following inflammation, increased axonal transport and peripheral biosynthesis of TRPV 1 occurs. ${ }^{4}$ The increased biosynthesis of TRPV 1 in nerve terminals may contribute to an augmented visceral sensory discharge and symptom generation. We have not examined the exact location of the TRPV 1 mRNA transcripts in the mucosal samples. We assume that TRPV 1 mRNA in the mucosa is derived from the TRPV 1-positive extrinsic afferents projecting in the mucosa representing processes of spinal afferents and, in more proximal parts of the intestine, of some vagal afferents. ${ }^{16-18}$ This hypothesis is supported by the observation that TRPV 1 mRNA disappears from the rat gastric wall following total extrinsic denervation of the stomach. ${ }^{17}$

The involvement of neuropeptides, among which SP released by TRPV 1-positive nerve terminals upon activation, has also been postulated to play a role in mediating visceral nociception. ${ }^{19}$ We here provide evidence for elevated mucosal concentrations of SP in IBS patients compared to UC patients. However, we found no correlation between pain 
symptom severity and mucosal SP concentrations. This finding is supported by recent observations of Kerckhoffs et al., who also found elevated mucosal levels of SP in IBS, but did not find any correlation with visceral hypersensitivity as determined by rectal distensions. ${ }^{20}$ Similarly, substance $P$ immunoreactive fibres were shown to be increased in mucosal samples of IBS patients compared to controls, but this was not related to abdominal pain.?

A possible explanation for these observations is that the release of substance $P$ is probably not the sole mechanism responsible for pain generation in IBS. Viscerosensation at mucosal level is largely under influence of redundant local mechanisms involving other signaling molecules, such as serotonin, norepinephrine, CGRP. Even more so, pain generation is a complex neurobiological process, which is largely influenced by other than mucosal processes, such as central pain processing at spinal and cortical levels, as well as influenced by the autonomic nervous system. This concept is in line with the current hypotheses on visceral hypersensitivity in IBS, emphasizing that alterations in the modulatory balance of pro- and anti-nociceptive local (mucosal) and central processing of peripheral input is paramount in determining the overall sensory perception of an individual. ${ }^{1}$ This is especially relevant in this patient population, which is often characterized by frequent psychiatric comorbidity (anxiety and/or depression). Future studies should therefore be aimed at delineating predominant pathogenetic mechanisms in the development of visceral pain conditions.

In order to assess the potential role of a preceding mucosal inflammation in visceral pain generation, we examined UC patients in remission. Evidence supports the role for TRPV 1 and SP in the pathogenesis of active inflammation in UC., 21-23 In fact, the density of SPcontaining nerve terminals in the lamina propria of active UC patients correlates with the severity of disease: a decrease in substance $P$ immunoreactivity was observed when the active disease became inactive, and an increase upon disease reactivation. ${ }^{24}$ In this study, we observed no significant increase in mucosal concentrations of SP, which is not surprising, as all patients were in endoscopic and microscopic remission, and only a 
minority (32\%) had symptoms such as abdominal pain and cramping. In UC patients reporting pain symptoms, a positive correlation was found with the transcription of TRPV 1 receptor, but not with mucosal SP concentrations, an observation also seen in the IBS group. This is in line with findings of Akbar et al. who demonstrated that UC patients in remission complaining of abdominal pain had significantly higher number of TRPV 1posititive nerve fibres in the rectosigmoid mucosa. ${ }^{25}$ Overall, these results support the hypothesis that altered nociception in UC patients in remission complaining of abdominal pain and IBS patients may share similar pathophysiology: an initial event, such as mucosal inflammation, can induce a change in the neurobiology of pain perception, resulting in altered peripheral pain processing and increased pain generation even when the inflammation has resolved.' We postulate that post-inflammatory upregulation of the TRPV 1 receptor through increased biosynthesis in the nerve terminals of mucosal afferents resulting in increased nociceptive sensitivity represents a mechanism potentially responsible for such sustained effect.

In our study, we also measured concentrations of the anti-nociceptive neuropeptide somatostatin, which is also released upon activation of the TRPV 1 receptor from mucosal nerve terminals. ${ }^{10} \mathrm{It}$ is also important to note, that nerve terminals are not the sole source of somatostatin in the mucosa, as large amounts can also be found in enteroendocrine cells. ${ }^{26}$ In UC patients, we found no significant changes in somatostatin concentrations. Although altered somatostatin levels in active UC have been reported, ${ }^{27}$ a pathophysiological role for somatostatin seems unlikely, as trials using somatostatin analogues have failed to show clear beneficial effects. ${ }^{28,29}$ On the other hand, we report here that IBS patients exhibit higher colonic mucosal levels of somatostatin. Somatostatin has been described to exert tonic analgesic control of peripheral nociceptors by modulating the TRPV 1 receptor. ${ }^{30}$ Therefore, elevated mucosal levels of somatostatin may potentially represent an increase in this tonic inhibitory control, aiming to counteract pro-nociceptive processes in the mucosa. In mucosal samples of IBS patients, the increase in somatostatin concentrations appeared to be relatively higher compared to SP. This increase is not necessarily related to increased TRPV1 activation, as other than 
neural sources of somatostatin have also been described in the intestinal mucosa. ${ }^{31}$ Apparently, this increase in mucosal somatostatin, whichever the source, is insufficient to counter-balance potential mucosal pro-nociceptive mechanisms as the majority of patients complain of abdominal pain. The fact that no correlation was found between neuropeptide concentrations and pain symptoms suggest the involvement of other mechanisms and may very well reflect the complexity of pain generation in IBS. Therefore, it remains to be elucidated whether this apparent dysregulation in neuropeptide concentrations has a legitimate role in the pathogenesis of altered nociceptive conditions in IBS.

It is important to note that our study has a number of limitations. Analyses were conducted in relatively small patient population. Also, due to the current lack of validated questionnaires for visceral pain, the questionnaires used in this study were not able to fully characterize visceral pain perception, and mainly focused on measuring pain intensity. Furthermore, the duration of disease and therefore symptom history is difficult to establish in IBS patients, as there may be a significant delay between diagnosis and onset of symptoms. Our study results will therefore need future substantiation.

In conclusion, our study demonstrates an increased transcription of TRPV 1 in mucosal samples of IBS patients, which correlated with pain symptom severity. This provides evidence for a role of TRPV 1 in pain generation in IBS and underlines a potential role for inflammation is developing sustained augmented pain perception. While the neuropeptides SP and somatostatin are both increased in IBS, these alterations seem insufficient to explain pain symptom generation in IBS patients. Pain is likely to be explained not only by a complex redundancy in the regulation of local nociceptive mechanisms, but also extensive central nociceptive processing, which remains to be subject of intensive further investigations. 


\subsection{REFERENCES}

1. Keszthelyi D, Troost FJ, Simren M, Ludidi S, Kruimel JW, Conchillo JM, Masclee AA. Revisiting concepts of visceral nociception in irritable bowel syndrome. Eur J Pain 2012.

2. Akbar A, Yiangou Y, Facer P, Walters JR, Anand P, Ghosh S. Increased capsaicin receptor TRPV 1-expressing sensory fibres in irritable bowel syndrome and their correlation with abdominal pain. Gut 2008;57:923-9.

3. Szallasi A, Nilsson S, Farkas-Szallasi T, Blumberg PM, Hokfelt T, Lundberg JM. Vanilloid (capsaicin) receptors in the rat: distribution in the brain, regional differences in the spinal cord, axonal transport to the periphery, and depletion by systemic vanilloid treatment. Brain Res 1995;703:175-83.

4. Tohda C, Sasaki M, Konemura T, Sasamura T, Itoh M, Kuraishi Y. Axonal transport of VR1 capsaicin receptor mRNA in primary afferents and its participation in inflammation-induced increase in capsaicin sensitivity. J Neurochem 200 1;76:162835.

5. Isgar B, Harman M, Kaye MD, Whorwell PJ. Symptoms of irritable bowel syndrome in ulcerative colitis in remission. Gut 1983;24:190-2.

6. Simren M, Axelsson J, Gillberg R, Abrahamsson H, Svedlund J, Bjornsson ES. Quality of life in inflammatory bowel disease in remission: the impact of IBS-like symptoms and associated psychological factors. Am J Gastroenterol 2002;97:389-96.

7. Yiangou Y, Facer P, Dyer NH, Chan CL, Knowles C, Williams NS, Anand P. Vanilloid receptor 1 immunoreactivity in inflamed human bowel. Lancet 2001;357:1338-9.

8. Holzer P. Transient receptor potential (TRP) channels as drug targets for diseases of the digestive system. Pharmacol Ther 2011;131:142-70.

9. Geppetti P, Trevisani M. Activation and sensitisation of the vanilloid receptor: role in gastrointestinal inflammation and function. Br J Pharmacol 2004;141:1313-20.

10. Pinter E, Helyes Z, Szolcsanyi J. Inhibitory effect of somatostatin on inflammation and nociception. Pharmacol Ther 2006;112:440-56.

11. Green PG, Basbaum Al, Levine JD. Sensory neuropeptide interactions in the production of plasma extravasation in the rat. Neuroscience 1992;50:745-9.

12. van der Heide H, van den Brandt-Gradel V, Tytgat GN, Endert E, Wiltink EH, Schipper ME, Dekker W. Comparison of beclomethasone dipropionate and prednisolone 21phosphate enemas in the treatment of ulcerative proctitis. $J$ Clin Gastroenterol 1988; 10:169-72.

13. Hamer HM, Jonkers DM, Vanhoutvin SA, Troost FJ, Rijkers G, de Bruine A, Bast A, Venema K, Brummer RJ. Effect of butyrate enemas on inflammation and antioxidant status in the colonic mucosa of patients with ulcerative colitis in remission. Clin Nutr 2010;29:738-44.

14. Nemeth J, Oroszi G, Than M, Helyes ZS, Pinter E, Farkas B, Szolcsanyi J. Substance $P$ radioimmunoassay for quantitative characterization of sensory neurotransmitter release. Neurobiology (Bp) 1999;7:437-44.

15. Nemeth J, Helyes Z, Gorcs T, Gardi J, Pinter E, Szolcsanyi J. Development of somatostatin radioimmunoassay for the measurement of plasma and tissue contents of hormone. Acta Physiol Hung 1996;84:313-5. 
16. Patterson LM, Zheng H, Ward SM, Berthoud HR. Vanilloid receptor (VR1) expression in vagal afferent neurons innervating the gastrointestinal tract. Cell Tissue Res 2003;311:277-87.

17. Schicho R, Florian W, Liebmann I, Holzer P, Lippe IT. Increased expression of TRPV 1 receptor in dorsal root ganglia by acid insult of the rat gastric mucosa. Eur $\mathrm{J}$ Neurosci 2004;19:1811-8.

18. Ward SM, Bayguinov J, Won KJ, Grundy D, Berthoud HR. Distribution of the vanilloid receptor (VR1) in the gastrointestinal tract. J Comp Neurol 2003;465:121-35.

19. Bueno L, Fioramonti J, Garcia-Villar R. Pathobiology of visceral pain: molecular mechanisms and therapeutic implications. III. Visceral afferent pathways: a source of new therapeutic targets for abdominal pain. Am J Physiol Gastrointest Liver Physiol 2000;278:G670-6.

20. Kerckhoffs AP, Ter Linde JJ, Akkermans LM, Samsom M. SERT and TPH-1 mRNA expression are reduced in Irritable Bowel Syndrome patients irrespective of visceral sensitivity state in large intestine. Am J Physiol Gastrointest Liver Physiol 2012.

21. Goode T, O'Connell J, Anton P, Wong H, Reeve J, O'Sullivan GC, Collins JK, Shanahan F. Neurokinin-1 receptor expression in inflammatory bowel disease: molecular quantitation and localisation. Gut 2000;47:387-96.

22. Bernstein CN, Robert ME, Eysselein VE. Rectal substance $P$ concentrations are increased in ulcerative colitis but not in Crohn's disease. Am J Gastroenterol 1993;88:908-13.

23. Keranen U, Kiviluoto T, Jarvinen H, Back N, Kivilaakso E, Soinila S. Changes in substance P-immunoreactive innervation of human colon associated with ulcerative colitis. Dig Dis Sci 1995;40:2250-8.

24. Watanabe T, Kubota Y, Muto T. Substance $P$ containing nerve fibers in rectal mucosa of ulcerative colitis. Dis Colon Rectum 1997;40:718-25.

25. Akbar A, Yiangou Y, Facer P, Brydon WG, Walters JR, Anand P, Ghosh S. Expression of the TRPV 1 receptor differs in quiescent inflammatory bowel disease with or without abdominal pain. Gut 2010;59:767-74.

26. Penman E, Wass JA, Butler MG, Penny ES, Price J, Wu P, Rees LH. Distribution and characterisation of immunoreactive somatostatin in human gastrointestinal tract. Regul Pept 1983;7:53-65.

27. Yamamoto H, Morise K, Kusugami K, Furusawa A, Konagaya T, Nishio Y, Kaneko H, Uchida K, Nagai H, Mitsuma T, Nagura H. Abnormal neuropeptide concentration in rectal mucosa of patients with inflammatory bowel disease. J Gastroenterol 1996;31:525-32.

28. Van Assche G, Ferrante M, Vermeire S, Noman M, Rans K, Van der Biest L, Penninckx F, Wolthuis A, Rutgeerts P, D'Hoore A. Octreotide for the treatment of diarrhoea in patients with ileal pouch anal anastomosis: a placebo-controlled crossover study. Colorectal Dis 2012;14:e181-6.

29. van Bergeijk JD, Wilson JH, Nielsen OH, von Tirpitz C, Karvonen AL, Lygren I, Radler A, Waldum HL, Mulder CJ, Friis S, Tefera S, Hoogkamer JF. Octreotide in patients with active ulcerative colitis treated with high dose corticosteroids (OPUS 1). Eur $\mathrm{J}$ Gastroenterol Hepatol 2002;14:243-8.

30. Carlton SM, Zhou S, Du J, Hargett GL, Ji G, Coggeshall RE. Somatostatin modulates 
the transient receptor potential vanilloid 1 (TRPV1) ion channel. Pain 2004;1 10:61627.

31. Teitelbaum DH, Del Valle J, Reyas B, Post L, Gupta A, Mosely RL, Merion R. Intestinal intraepithelial lymphocytes influence the production of somatostatin. Surgery 1996; 120:227-32; discussion 232-3. 
TRPV1 and neuropeptides in pain generation 
Impaired barrier function in Irritable Bowel Syndrome: a key factor to serotonin-modulated visceral hypersensitivity

D. Keszthelyi, FJ. Troost, DM. Jonkers, J. Dekker, H. van Eijk, PJ. Lindsey, WA. Buurman, AA. Masclee

Part of this chapter has been published in Alimentary Pharmacology and Therapeutics 2014

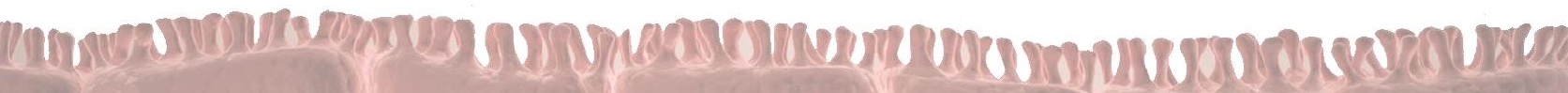


Part III. The putative relationship between intestinal barrier function, serotonin metabolism and visceral hypersensitivity in IBS

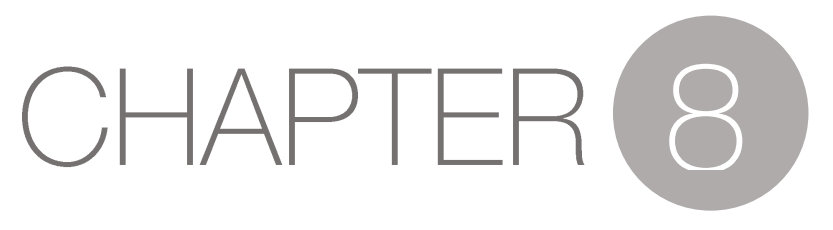

Mechanistic insight using the serotonin precursor

5-hydroxytryptophan

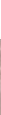




\section{ABSTRACT}

Background \& Aims: Altered serotonergic (5-HT) metabolism, visceral perception and impaired intestinal barrier function have been associated with the pathogenesis of irritable bowel syndrome (IBS). We hypothesized that the direct precursor of 5-HT, 5hydroxytryptophan (5-HTP), modulates 5-HT metabolism and consequently affects visceral perception and intestinal barrier function.

Methods: 15 IBS patients and 15 healthy volunteers recruited at the Maastricht University Medical Center participated in this randomized double-blind placebo controlled study. The $100 \mathrm{mg}$ 5-HTP or placebo was ingested orally. Serotonergic metabolites were assessed in platelet poor plasma and duodenal mucosal samples. Visceroperception was measured by rectal barostat. Intestinal barrier function was assessed using a dual-sugar test and tight junction protein transcription and expression in duodenal mucosa.

Results: 5-HTP induced an increase in systemic and mucosal serotonergic metabolites in both healthy controls and IBS patients. 5-HTP also induced a pro-nociceptive response, which was most apparent in hypersensitive patients. Administration of 5-HTP resulted in increased barrier function in healthy controls, whereas the opposite effect was seen in IBS. Patients with IBS had an impaired barrier function, regardless whether patients were found normo- or hypersensitive.

Conclusion: IBS patients demonstrated an increased perceptive and metabolic response to 5-HTP accompanied by a failure to reinforce intestinal integrity. We postulate therefore that hypersensitivity in IBS develops as a consequence of impaired barrier function. Our data contribute to a better understanding of serotonergic signaling, in particular in relation to changes in intestinal integrity and visceral hypersensitivity in the pathophysiology of IBS and may pave ways to develop novel therapeutic modalities in functional gastrointestinal disorders such as IBS. 


\subsection{INTRODUCTION}

Irritable bowel syndrome (IBS) is a common gastrointestinal disorder affecting up to $15 \%$ of the Western population. It is characterized by lower abdominal discomfort or pain with disturbed defecation in the absence of apparent structural or biochemical abnormalities that might explain the symptoms.' Despite the high prevalence of IBS, the pathophysiology is still poorly understood due to the heterogeneous nature of the disorder. Consequently, IBS currently lacks effective therapeutic approaches.

Previous studies have pointed to a number of alterations in intestinal physiology in IBS. First, alterations in serotonergic metabolism have been associated with IBS. ${ }^{2}$ Serotonin (5-hydroxytryptamin, 5-HT) has an important role in regulating human gastrointestinal function, including intestinal sensing and signaling. ${ }^{3}$ The alterations in serotonergic metabolism may therefore lead to profound changes in intestinal function and contribute to IBS pathogenesis. Recent evidence suggests an important role for the duodenum with respect to abnormal mucosal $5-\mathrm{HT}$ metabolism in IBS. ${ }^{4}$

Second, it is now widely accepted that an altered visceral sensitivity through abnormal endogenous pain processing plays an important role in the pathogenesis of IBS. ${ }^{5}$ Although not all IBS patients exhibit visceral hypersensitivity, increased sensory responses to rectal balloon distension have been reported in up to $60 \%$ of the patients.

Third, accumulating evidence points to impaired intestinal barrier function in IBS, documented in 12-50\% of IBS patients by sugar permeability tests. ${ }^{6}$ In addition, recent reports suggest abnormalities in tight junction proteins of both colonic ${ }^{7}$ and small intestinal (jejunal) tissue 8,9 of IBS patients. An impairment of barrier function has been postulated to facilitate the passage of potentially noxious luminal substances into the lamina propria, leading to mucosal immune activation, with subsequent sensitization of nerve endings generating abdominal complaints. ${ }^{10}$ Increased permeability has also been shown to be associated with increased sensory responses to visceral stimuli. ${ }^{11}$

In an attempt to gain more insight in the pathophysiology of IBS, we sought to answer 


\section{Chapter 8}

the question whether differences in serotonergic signaling underlie the initiation of a cascade of events leading to a reduced epithelial integrity and increased visceral pain perception. The present study investigates the relation between 5-HT metabolism, intestinal barrier function and visceral perception both in healthy controls and IBS patients. To this end, we measured local and systemic serotonin metabolites, tested visceral perception using rectal balloon distensions, used a dual-sugar permeability test and assessed duodenal mucosal expression and transcription of tight junction proteins.

\subsection{METHODS}

The study was approved by the Medical Ethics Committee of the Maastricht University Medical Centre+ and was conducted according to the revised version of the Declaration of Helsinki (October 2008, Seoul). All volunteers gave their written informed consent prior to participation. The study has been registered at the US National Library of Medicine (http://www.clinicaltrials.gov, NCT00731003). All authors had access to the study data and had reviewed and approved the final manuscript.

\section{PARTICIPANTS}

Fifteen healthy volunteers and 15 patients with IBS diagnosed according to the Rome III criteria were included in this randomized double-blind placebo-controlled cross-over study (see supplementary material for sample size calculation). All participants completed the study. All subjects were screened involving a standardized general physical examination. Exclusion criteria included history of gastrointestinal disorders (other than IBS) or psychiatric disorders including use of psychoactive medication, use of medication affecting gastrointestinal function (including NSAIDs) or serotonergic metabolism within 14 days prior to testing. Patients fulfilling the Rome III criteria for functional dyspepsia were excluded from the study. During the screening procedure, participants were familiarized with the rectal distension procedure by inserting a rectal barostat catheter following a self-administered rectal saline enema $(50 \mathrm{~mL})$ and applying three random distensions at different pressure levels. 


\section{STUDY DESIGN}

The study included four test days (first two test days for visceroperception and a second two test days for intestinal permeability/serotonin metabolism), which were all separated by a minimum wash-out period of seven days (see supplementary Figure 1). For each of the two test days, participants received either 5-HTP or placebo based on a random preselection at 8:00 AM following an overnight fast. Randomisation was performed by the Pharmacy Department of our hospital.

All subjects were tested within three months to avoid possible seasonal variation. Participants were requested to abstain from heavy physical exercise and consumption of alcohol and of tryptophan-rich food the day prior to their visit.

Study medication containing $100 \mathrm{mg}$ 5-HTP was obtained from Tiofarma BV, Oud Beijerland, the Netherlands, and prepared by the Department of Pharmacy of our hospital. Identical tablets without 5-HTP were used as placebo medication. Previous studies indicated that $100 \mathrm{mg}$ of 5 -HTP was well tolerated without serious side effects and has elimination half-life of 1.5 hours. ${ }^{12}$ In our study nausea and/or belching was experienced by three healthy volunteers and three IBS patients following 5-HTP intake. Furthermore, two IBS patients complained of abdominal pain, one of bloating, one of diarrhea following intake of 5-HTP. No side effects were experienced following placebo intake.

\section{Rectal sensitivity test}

One hour following 5-HTP or placebo intake, visceroperception was measured by phasic distensions using a rectal barostat (Distender II; GEJ Electronics, Toronto, Canada) as described previously ${ }^{13}$, see also supplementary material. Pain sensation was scored using a 100-mm visual analogue scale (VAS). 


\section{Intestinal permeability test}

One hour following 5-HTP or placebo ingestion, participants received an oral sugar drink to assess intestinal permeability. The sugar drink consisted of $1 \mathrm{~g}$ lactulose (Centrafarm Services, Etten-Leur, the Netherlands) and $0.5 \mathrm{~g}$ L-rhamnose (Danisco Sweeteners, Thomson, IL). Blood samples were taken following 60 minutes of sugar ingestion. Directly hereafter, participants underwent a gastroduodenoscopy performed by an experienced gastroenterologist. Eight mucosal samples from the horizontal part of the duodenum were obtained by standard gastroduodenoscopy using standard forceps (diameter $2.8 \mathrm{~mm}$ ) and immediately frozen in liquid nitrogen. Two of the samples were imbedded in Tissue-tek ${ }^{\circledR}$ (Sakura Finetek, Tokio, Japan) prior to freezing for immunohistochemical analyses.

\section{Assessment of plasma sugar concentrations}

Samples for plasma were collected using pre-chilled K2EDTA tubes prior to, and 60 after ingestion of the sugar drink. The tubes were centrifuged at $3000 \mathrm{~g}$ at $4^{\circ} \mathrm{C}$ for 10 minutes. Plasma was aliquoted and immediately frozen at $-80^{\circ} \mathrm{C}$ until analysis. Plasma sugar concentrations were determined in the $t=60$ min samples with correction for initial sugar concentrations in the fasted blood samples by HPLC-MS, as described by van Wijck et al. ${ }^{14}$ Ratios for lactulose/L-rhamnose were calculated to indicate small intestinal permeability, which has been validated recently. ${ }^{14}$

\section{Expression of tight junction proteins in mucosal samples}

Gene transcription of tight junction proteins (zonula occludens-1, ZO-1, and occludin) in biopsy specimens was measured by qRT-PCR. Glyceraldehyde 3-phosphate dehydrogenase (GAPDH) and 18S RNA were used as reference genes (see also supplementary material).

Immunofluorescent staining for ZO-1 and occludin was performed on frozen sections of the biopsy specimens (see also supplementary material). To quantify the fluorescent 
staining of ZO-1 and occludin in the $\mathrm{TJ}$ region, each coded tissue sample was subjected to confocal analysis of uniform $Z$ sections perpendicular to the apical cell surface of the epithelium in 3 different areas per section (Figure 1A). These areas were selected randomly within three different $Z$ sections. Staining was analyzed in the medium region of the villi. Plot profiles (e.g., insert in Figure $1 A$ ) of the staining intensity along the perpendicular lines were generated using Image $\mathrm{J}$ software (National Institutes of Health, Bethesda, MD), with the LSM toolbox plug-in. The fluorescent intensities were plotted as a function of the cell location using the peak fluorescence signal from the tight junction region to align each intensity profile beginning from the apical side of the cell.

\section{Assessment of systemic and mucosal 5-HT metabolism}

Samples for plasma and platelet poor plasma were collected using pre-chilled $K_{2}$ EDTA tubes prior to, and 60 and 120 min after administration of 5-HTP or placebo. To avoid oxidative breakdown, $1 \mathrm{ml} 1.4 \%$ ascorbic acid (Sigma Aldrich, St. Louis, MO) was added to tubes for 5-HT analysis. Platelet poor plasma collection tubes were centrifuged at 2000 $\mathrm{g}$ at $4^{\circ} \mathrm{C}$ for 10 minutes. Supernatants were allocated and immediately frozen at $-80^{\circ} \mathrm{C}$ until analysis. Biopsy specimens were weighed and homogenized prior to analysis. Concentrations of 5-HTP, 5-HT, 5-HIAA mucosa were determined by HPLC-MS as described by previously (see also supplementary material). ${ }^{15}$

\section{Statistical analyses}

Statistical analyses were performed using SPSS 20 (SPSS Inc., Chicago IL). Data were tested for normality by the Kolmogorov-Smirnov test. Normally distributed data were analyzed by Student's test and two-way ANOVA. Wilcoxon, Mann-Whitney U-test, Kruskal-Wallis test and Friedman test (for repeated measures) were used for nonparametric data. Coefficients for correlations were calculated according to Pearson and Spearman, respectively. Data expressed as mean \pm SEM or as median (range), unless otherwise stated. PCR data are presented as expression normalized for GAPDH and $18 \mathrm{~S}$ RNA. 


\section{Chapter 8}

Data analysis from the immunohistochemical staining were performed in the software application 'R' using the elliptic library ${ }^{16}$ by a multivariate non-linear Gaussian regression in order the integrate the plot profiles of the staining intensities and to ascertain the differences in staining intensities for ZO-1 and occludin between the two treatment conditions (see supplementary data). Confidence intervals were calculated on basis of the maximum and minimum difference in staining intensities between the two treatments (5HTP and placebo).

Thresholds for pain perception were defined as the pressure step during distension when the VAS score reached or surpassed $10 \mathrm{~mm}$. In case scores were not higher than $10 \mathrm{~mm}$ in the distention range used in the study, the pain threshold was defined as the maximum pressure $(50 \mathrm{mmHg}$ ). Hypersensitivity to rectal distension was defined as a pain threshold below $26 \mathrm{mmHg} .^{17}$

\subsection{RESULTS}

\section{DEMOGRAPHIC CHARACTERISTICS}

IBS patients did not differ significantly from healthy controls regarding age $(33 \pm 17$ yrs vs $44 \pm 13$ yrs, $p=0.06$ ), gender ( $46 \%$ male vs $33 \%$ male, $p=0.35$ ) or $\mathrm{BMl}(24 \pm 4$ vs $27 \pm 5$, $p=0.08$, healthy vs IBS, respectively). IBS subtypes were $47 \%$ diarrhea predominant, 33\% constipation predominant, $20 \%$ mixed subtype. No association was found between age, gender or IBS subtype and any of the parameters studied.

\section{INCREASED BASELINE SYSTEMIC CONCENTRATIONS OF 5-HT AND 5-HIAA IN IBS}

Two aspects of serotonergic metabolism were assessed: 1) baseline differences between IBS patient and controls and 2) differential response to 5-HTP administration. Therefore, systemic and mucosal concentrations of 5-HTP, 5-HT and 5-HIAA, the main metabolic breakdown product of $5-\mathrm{HT}$, were measured (Figure 2).

Baseline platelet poor plasma concentrations of $5-\mathrm{HT}(1.9 \pm 0.4 \mathrm{nmoV} / \mathrm{vs} 29.5 \pm 5.6$, healthy 
vs IBS) and 5-HIAA (5.1 $\pm 0.4 \mathrm{nmoV} /$ vs $15.1 \pm 1.8$, healthy vs IBS) were significantly higher in IBS (both p<0.001). Ratios for 5-HIAA/5-HT, assumed to reflect 5-HT turnover, were significantly lower in IBS compared to controls $(3.3 \pm 1.0$ vs $0.95 \pm 0.28$, healthy vs IBS, $\mathrm{p}=0.01$ ). This potentially reflects impaired uptake of $5-\mathrm{HT}$ into platelets, as proposed by Foley et al. ${ }^{4}$

\section{5-HTP INDUCES A LARGER INCREASE IN SYSTEMIC 5-HIAA LEVELS IN IBS}

Next, we examined the effects of 5-HTP administration healthy controls and IBS patients. In healthy controls, administration of 5-HTP led to significantly increased levels of platelet poor plasma 5 -HTP ( $54 \pm 8$ vs $0.9 \pm 0.1 \mathrm{nmoV}$, 5 -HTP vs placebo, 60 minutes after intake, p<0.000 1), but did not affect $5-H T$ levels $(7.3 \pm 3.7$ vs $5.0 \pm 2.6 \mathrm{nmol} /, 5-H T P$ vs placebo $\mathrm{p}=0.64$ ), whereas 5 -HIAA levels showed a significant 100 -fold increase $(316 \pm 28$ versus $5 \pm 1 \mathrm{nmoV}, 5-\mathrm{HTP}$ vs placebo, $\mathrm{p}<0.0001$ ), Figure 2 . Therefore, $5-\mathrm{HIAA} / 5-\mathrm{HT}$ ratio was significantly increased following 5 -HTP intake $(192 \pm 56$ vs $4.6 \pm 1.4 \mathrm{nmol}$, $5-\mathrm{HTP}$ vs placebo, $\mathrm{p}<0.0001)$, indicative of increased $5-\mathrm{HT}$ turnover.

In IBS patients, more pronounced effects were observed on systemic serotonin metabolites following 5-HTP intake; a significant increase in 5-HTP concentrations ( $252 \pm 30$ vs $1.5 \pm 0.3 \mathrm{nmoVl}, 5$-HTP vs placebo, 60 minutes after intake, $p<0.0001)$, as well as a nearly 1000 -fold increase in 5 -HIAA concentrations ( $1081 \pm 127$ vs $14 \pm 2 \mathrm{nmol} / \mathrm{l}, 5-$ HTP vs placebo, p<0.0001) was observed. $5-H T$ levels remained unaffected $(32.4 \pm 10.3$ vs $27.3 \pm 3.5 \mathrm{nmoV} /, 5-\mathrm{HTP}$ vs placebo, $\mathrm{p}=0.52$ ). Hence, 5 -HIAA/5-HT ratios were significantly higher following $5-\mathrm{HTP}$ intake in IBS $(99 \pm 27$ vs $0.73 \pm 0.17,5-\mathrm{HTP}$ vs placebo, $p=0.005$ ).

The magnitude of the 5-HTP-induced increase in the concentration of the metabolites was significantly higher in IBS patients than in healthy controls; two-way analysis of ANOVA of the 5-HTP and 5-HIAA concentrations at 60 minutes showed a significant effect of the condition (IBS or healthy), treatment (5-HTP or placebo), as well as the interaction (all $p<0.001$ ). This suggests the unexpectedly high 5 -HIAA increase to be at 


\section{Chapter 8}

least in part due to increased baseline 5-HT turnover in IBS.

\section{BASELINE MUCOSAL TURNOVER OF 5-HT IN INCREASED IN IBS}

Baseline mucosal concentrations of $5-\mathrm{HT}$ were significantly lower in IBS $(60 \pm 21 \mathrm{pmol} / \mathrm{mg}$ vs $13 \pm 2$, healthy vs IBS, $p=0.004$ ), while baseline 5 -HIAA concentrations were significantly higher $(2.5 \pm 0.7 \mathrm{pmol} / \mathrm{mg}$ vs $8.1 \pm 1.3$ vs, healthy vs IBS, $p=0.001)$. This may reflect increased mucosal conversion of 5-HT to 5-HIAA, as also reflected by significantly higher mucosal 5-HIAA/5-HT ratios in IBS $(0.31 \pm 0.2$ vs $2.0 \pm 0.5$, healthy vs IBS, p<0.005).

\section{EXOGENOUS 5-HTP DOES NOT FURTHER ENHANCE MUCOSAL 5-HT TURNOVER IN IBS}

After 5-HTP administration, mucosal 5-HTP concentrations in healthy volunteers were significantly higher compared to placebo $(3.7 \pm 1.8$ vs $0.09 \pm 0.03 \mathrm{pmol} / \mathrm{mg}, 5-\mathrm{HTP}$ vs placebo, $p=0.005)$. Such effect was not seen in IBS patients $(1.1 \pm 0.9$ vs $0.09 \pm 0.03$ pmol/mg, $\mathrm{p}=0.26$ ). 5-HTP intake did not affect mucosal 5-HT concentrations in neither healthy volunteers $(60 \pm 21$ vs $52 \pm 18 \mathrm{pmol} / \mathrm{mg}, 5-\mathrm{HTP}$ vs placebo, $\mathrm{p}=0.68)$, nor IBS patients ( $13 \pm 2$ vs $13 \pm 3 \mathrm{pmol} / \mathrm{mg}, 5-\mathrm{HTP}$ vs placebo $\mathrm{p}=0.90)$. Mucosal concentrations of 5-HIAA were significantly increased in healthy volunteers after 5-HTP compared to placebo (7.1 \pm 1.7 versus $2.5 \pm 0.7 \mathrm{pmol} / \mathrm{mg}, 5-\mathrm{HTP}$ vs placebo, $\mathrm{p}=0.02)$. Similar changes were observed in IBS (20.0 \pm 4.8 versus $8.1 \pm 1.3 \mathrm{pmoV} / \mathrm{mg}, 5$-HTP vs placebo, $\mathrm{p}=0.02$; Figure 2).

Interestingly, mucosal 5-HIAA/5-HT ratios remained similar following 5-HTP in both healthy controls $(0.47 \pm 0.3$ vs $0.31 \pm 0.2,5-H T P$ vs placebo, p>0.05) and IBS patients (3.2 \pm 1.1 vs $2.0 \pm 0.5,5$-HTP vs placebo, p>0.05). This implies that the condition and the treatment both significantly affect 5-HIAA concentrations, while there is no additive effect of the concomitant presence of both factors. Indeed, mucosal 5-HIAA levels observed in healthy controls after 5-HTP were comparable to those seen in IBS following placebo. 


\section{MUCOSAL 5-HIAA IS RAPIDLY CLEARED TO THE SYSTEMIC COMPARTMENT}

To gain further insight on 5-HT metabolism, we examined the relation between mucosal and systemic concentrations of 5-HT and 5-HIAA. Mucosal 5-HT concentrations were negatively correlated with mucosal 5-HIAA concentrations following placebo $(r=-0.50$, $p=0.01)$ and $5-H T P(r=-0.56, p=0.003)$, when both groups were analyzed together, suggesting an inverse relation between the two. Mucosal 5-HIAA concentrations correlated significantly with systemic 5-HIAA levels $(r=0.60, p=0.003)$, suggesting that 5HIAA produced in the mucosa is rapidly taken up by the systemic compartment, resulting in increased plasma 5-HIAA concentrations.

Taken together, data on mucosal serotonin metabolism suggest that 1) administration of 5-HTP results in increased production of 5-HIAA through 5-HT, 2) exogenous 5-HTP does not per se induce further acceleration of mucosal 5-HT turnover and 3) rapid clearance of 5-HIAA from the gut results in high systemic concentrations of 5-HIAA.

5-HTP INDUCES A PRO-NOCICEPTIVE RESPONSE TO RECTAL DISTENSION IN HYPERSENSITIVE IBS PATIENTS

We assessed visceral perception in healthy controls and IBS, in particular the potential effects of 5-HTP. IBS patients are known to have lower thresholds to rectal distension than healthy controls. Indeed, when comparing baseline conditions, IBS patients had significantly lower pain thresholds and significantly higher VAS scores than healthy controls ( $47 \pm 2 \mathrm{mmHg}$ vs $34 \pm 5 \mathrm{mmHg}, p=0.02 ; 14.2 \pm 4.0$ vs $3.5 \pm 0.7 \mathrm{~mm}$, healthy vs IBS, $p=0.01$, Figure 3). Hypersensitive subjects were only detected among patients $(0 \%$ vs $46.6 \%, p=0.002$, healthy vs IBS). The proportion of hypersensitive patients was comparable to literature data. ${ }^{18}$

None of the healthy volunteers became hypersensitive following placebo intake. Six healthy volunteers showed a lower threshold for pain perception after 5-HTP (an allodynic reaction), 4 of which became hypersensitive $\left(x^{2}=4.5, p=0.03,5-H T P\right.$ vs placebo; Figure 3). On a group level, pressure thresholds for pain perception were therefore 


\section{Chapter 8}

significantly lower after 5-HTP (38 \pm 4 versus $47 \pm 2 \mathrm{mmHg}, 5$-HTP vs placebo, $\mathrm{p}=0.042$ ). VAS scores for pain perception were significantly higher after 5-HTP, but only in the pressure range of $35-50 \mathrm{mmHg}(10.8 \pm 2$ versus $6.8 \pm 1 \mathrm{~mm} ; \mathrm{p}=0.025)$.

Regarding 5-HTP and visceroperception in IBS, the proportion of hypersensitive IBS patients as well as pain thresholds remained unaltered by $5-\mathrm{HTP}(31 \pm 5$ vs $34 \pm 5 \mathrm{mmHg}$ on a group level, 5-HTP vs placebo, $\mathrm{p}=0.12$ ). On the other hand, mean VAS scores for pain were significantly higher following 5 -HTP in IBS patients $(17.2 \pm 4$ vs $14.2 \pm 4 \mathrm{~mm}$, $p=0.04$; Figure 3). The increased VAS score was significantly higher in hypersensitive IBS patients compared to normosensitive patients (increase in mean VAS $6.7 \pm 2$ vs $0.1 \pm 0.7$ mm, $\mathrm{p}=0.02$, see Figure 4 and supplementary Figure 2). Thus, already hypersensitive IBS patients became even more hypersensitive while normosensitive IBS patients did not become hypersensitive after 5-HTP intake.

\section{5-HTP ENHANCES INTESTINAL BARRIER FUNCTION IN HEALTHY CONTROLS BUT NOT IN} IBS

Previous studies have reported that $5-\mathrm{HT}$ affects intestinal epithelial permeability ${ }^{19}$ and that intestinal permeability is increased in IBS. Therefore, small intestinal barrier function was evaluated by administering a dual-sugar test.

Two-factor ANOVA analysis showed a significant effect of the condition (IBS or healthy, p<0.05), but not for the treatment or interaction, indicating that, as a group, IBS patients had increased L/R permeability compared to controls. In healthy controls, ratios for lactulose/L-rhamnose (L/R) were significantly lower following 5-HTP administration compared to placebo in controls $\left(4.9 \times 10^{-3} \pm 0.0005\right.$ vs $6.3 \times 10^{-3} \pm 0.0005, p=0.01$; Figure 5). This difference results from a decrease in lactulose concentration after 5-HTP vs placebo ( $1.3 \pm 0.5$ vs $1.7 \pm 0.6 \mu \mathrm{moV}, \mathrm{l}=0.035$ ), as no changes were observed in the L-rhamnose concentration ( $91 \pm 6$ vs $97 \pm 5 \mu \mathrm{moV}, \mathrm{p}=0.41$ ). In IBS patients, no significant change was observed in L/R ratios following 5-HTP administration (20x $10^{-3} \pm 0.009$ vs $12 \times 10^{-3} \pm 0.003$, 5-HTP vs placebo, $\mathrm{p}=0.89$ ), see Figure 5. 


\section{5-HTP INDUCES ZO-1 TRANSCRIPTION IN HEALTHY CONTROLS BUT NOT IN IBS}

To further characterize intestinal integrity, transcription of the tight junction proteins ZO-1 and occludin was examined in duodenal mucosa. No significant difference was observed in ZO-1 transcription between IBS and healthy controls in the placebo conditions ( $\mathrm{p}=0.24$; Figure $6 \mathrm{~A})$. Interestingly, 5-HTP administration resulted in significantly higher transcription of ZO-1 in healthy volunteers (normalized expression ratios $1.27 \pm 0.24$ vs $0.87 \pm 0.12,5$-HTP vs placebo, $p=0.04$ ), while in IBS patients, such increase in ZO- 1 transcription was not seen $(0.75 \pm 0.16$ vs $1.25 \pm 0.23,5$-HTP vs placebo, $\mathrm{p}=0.06$ ). Two-way ANOVA analysis showed a significant effect for the concomitant presence of treatment (5-HTP or placebo) and condition (IBS or healthy) $(\mathrm{p}=0.03)$, but not for treatment or condition alone ( $\mathrm{p}=0.81$ and 0.70 , respectively).

Transcription of the transmembrane tight junction protein occludin was significantly lower in IBS than in healthy controls on both conditions ( $p=0.006$ for placebo and $p=0.001$ for 5-HTP; Figure 6B). Administration of 5-HTP did not affect transcription of occludin neither in healthy controls nor in IBS patients (normalized expression ratios $1.43 \pm 0.14$ vs $1.39 \pm 0.12,5$-HTP vs placebo, $p=1.0 ; 0.57 \pm 0.14$ vs $0.80 \pm 0.15,5$-HTP vs placebo, $p=0.20$, respectively).

\section{5-HTP ALTERS THE INTRACELLULAR DISTRIBUTION OF ZO-1 PROTEIN}

In the placebo condition, expression of both ZO-1 and occludin, expressed as area under curve (AUC), was significantly lower in IBS patients compared to healthy controls. Administration of 5-HTP induced a decrease in the AUC of ZO-1 expression in healthy controls (see table insert in Figure 1). However, a significant shift in peak intensity of ZO-1 was observed in the direction of the apical surface of the cell, indicating increased staining intensity in this region, which corresponds to increased amounts of protein in the proximity of the tight junctions. In IBS, following 5-HTP, an apical shift in peak intensity for Z0-1 was observed similarly to that seen in healthy controls.

The expression of the transmembrane tight junction protein occludin was found to be 
statistically significantly lower following 5-HTP in healthy controls. However, given the minute magnitude of the change observed, the biological effect is most probably not essential. Also, a very similar curve shape was observed, indicating unaltered protein distribution.

In IBS, occludin was more diffusely distributed in the cytosol in the placebo condition, i.e. a substantial amount of the protein was located outside the direct proximity of the tight junction region. Remarkably, 5-HTP administration led to a decrease in overall occludin expression, with the majority of the protein localized to the tight junction region.

Taken together, the intestinal permeability data indicate an increased intestinal permeability compared in IBS to healthy controls. This appears to be the result of a primary defect of the intestinal barrier as reflected by decreased transcription and expression of tight junction proteins. Following 5-HTP, healthy controls demonstrate an increased barrier function, accompanied by increased transcription and redistribution of ZO-1. In IBS patients, 5-HTP administration led to a similar change in ZO-1 distribution, however, without enhanced barrier function.

\section{HYPERSENSITIVE IBS PATIENTS DEMONSTRATE HIGHER PLASMA 5-HIAA CONCENTRATIONS}

Next, we examined the relationship between serotonin metabolism, visceral perception and barrier function. We first investigated the putative relation between alterations in serotonergic metabolism and visceral perception. Mean pain scores significantly correlated with systemic 5-HIAA concentrations at $\mathrm{t}=60$ minutes, both following placebo $(r=0.650, p<0.0001)$ and 5-HTP $(r=0.402, p=0.042)$ when IBS patients and healthy controls were analyzed together. Hypersensitive IBS patients had significantly higher plasma 5HIAA concentrations at $\mathrm{t}=60$ minutes than normosensitive IBS patients following placebo (19.1 \pm 2.3 vs $12.3 \pm 1.8 \mathrm{nmol}, \mathrm{p}=0.03$; Figure 7 ) but not following $5-\mathrm{HTP}$ ( $1102 \pm 166$ vs $1057 \pm 228 \mathrm{nmol} /$, hypersensitive vs normosensitive, $\mathrm{p}=0.85$ ). This implies that baseline 5-HIAA could potentially predict perceptional status in IBS. 
Normosensitive IBS patients, although comparable to healthy controls in terms of visceral perception, had higher levels of $5-\mathrm{HT}$ in both placebo and $5-\mathrm{HTP}$ conditions ( $23.0 \pm 6.6$ vs $2.0 \pm 0.5 \mathrm{nmoV} / 5-\mathrm{HT}$, normosensitive IBS vs healthy after placebo, $p<0.001 ; 28.6 \pm 12.1$ vs $6.34 \pm 4.13 \mathrm{nmoV} / \mathrm{I} 5-\mathrm{HT}$, normosensitive IBS vs healthy after $5-\mathrm{HTP}, \mathrm{p}=0.02)$. The same holds true for 5 -HIAA ( $12.3 \pm 1.8$ vs $4.84 \pm 0.41 \mathrm{nmol} / 5$-HIAA, normosensitive IBS vs healthy after placebo, $p=0.02 ; 1057 \pm 228$ vs $342 \pm 33 \mathrm{nmol} / \mathrm{L}$-HIAA; normosensitive IBS vs healthy after $5-H T P, p=0.004$, see Figure 7 ). This implies that changes in serotonergic metabolism are a characteristic property of all IBS patients while alterations in perceptional status is restricted to the hypersensitive group.

Next, we assessed the relation between serotonin metabolism and barrier function. Here, a positive correlation between mucosal levels of 5-HIAA and the expression of ZO-1 mRNA in healthy controls $(r=0.70, p=0.008)$ and in IBS patients $(r=0.60, p=0.04)$ was found following 5-HTP. This is in line with the hypothesis that serotonergic signaling plays a role in barrier function reinforcement. No significant correlations were found between other parameters.

\section{A PRONOCICEPTIVE RESPONSE TO 5-HTP IS ACCOMPANIED BY INCREASED L/R RATIO}

Finally, we examined the relation between visceral perception and barrier function. No significant changes were observed with regards to ZO-1 or occludin transcription, expression or L/R ratio between hypersensitive and normosensitive IBS patients following placebo. This suggests that there is no significant difference between hyper- en normosensitive patients in baseline barrier function.

Interestingly, after 5-HTP administration, hypersensitive IBS and allodynic healthy controls showed higher $L / R$ ratios compared to normosensitive patients and healthy controls $(0.007 \pm 0.01$ vs $0.02 \pm 0.03$, normo- vs hypersensitive, $p=0.03)$. This suggests an important role for increased intestinal permeability in the development of a nociceptive reaction to 5-HTP. 


\subsection{DISCUSSION}

Several hypotheses have been formulated in the past with respect to the pathogenesis of IBS and in particular visceral hypersensitivity. In this regard, impaired intestinal barrier function is increasingly being appreciated as a causative factor by allowing potential luminal content to sensitize visceral afferents resulting in increased peripheral discharge, contributing to visceral hypersensitivity.6,20 We here investigated a potential role for serotonin, a key sensory modulator in the gut, in relation to this hypothesis.

To our knowledge, this is the first study to simultaneously assess serotonergic metabolism, visceral perception and intestinal barrier function in IBS patients in comparison to healthy controls. Here, we demonstrate that increased serotonergic metabolism, following oral administration of the serotonin precursor 5-HTP, results in an increase in visceral perception and induces an increase in intestinal mucosal barrier function in healthy individuals. In IBS patients, an increased perceptive and metabolic response to 5-HTP accompanied a failure to reinforce intestinal integrity. This impairment of the intestinal barrier was seen in both normosensitive and hypersensitive IBS patients, whereas the nociceptive response was seen only in hypersensitive patients.

Modulation of serotonergic metabolism by administering the serotonin precursor 5-HTP led to a significant increase in systemic and mucosal 5-HTP and 5-HIAA levels in both healthy controls and IBS patients. This is in line with previous observations, ${ }^{21}$ and is in indicative of an increase in 5-HT metabolism. Remarkably, 5-HT concentrations remained unchanged. This can be explained by the action of the serotonin transporter (SERT) on platelet membranes and intestinal epithelial cells, aimed to maintain steady state of systemic and mucosal concentrations of $5-\mathrm{HT}$, respectively. ${ }^{22}$

Interestingly, the administration of 5-HTP resulted in an unexpectedly high increase in 5HIAA concentrations in IBS patients. We assume that this is due to enhanced baseline metabolism in IBS patients. This led to the hypothesis that the excess availability of the precursor leads to augmented release of $5-\mathrm{HT}$ from enterochromaffin cells, which is 
rapidly taken up by SERT and converted to 5-HIAA. As the gut is the principal source of 5-HIAA in the human body, ${ }^{23}$ it is tempting to assume that the increase in both plasma and mucosal 5-HIAA seen following 5-HTP administration results from increased mucosal production of 5-HIAA and rapid clearance from the intestinal to the systemic compartment. In support of this hypothesis, Cremon et al. recently demonstrated increased 5-HT release from enterochromaffin cells in IBS patients. ${ }^{24}$

It is important to note, however, that findings with regards serotonin metabolism in IBS have been discrepant and consensus is lacking., 25-28 For instance, Dunlop et. al demonstrated decreased rectal mucosal 5-HIAA/5-HT ratios in IBS-C and post-infectious IBS patients. ${ }^{29}$ Duodenal 5-HIAA/5-HT ratios have not been investigated previously. Furthermore, due to the relatively small sample size, we were unable to assess potential differences between IBS subtypes. Moreover, we did not assess serotonin metabolism in more detail, such as regional mucosal differences or the activity of specific enzymes, for instance SERT, in platelets or mucosa. Neither were mast cells, another potential source of $5-\mathrm{HT}$ in the mucosa, subject to analysis in the current study.

Nonetheless, increased release of $5-\mathrm{HT}$ in the mucosa could provide an explanation for the pronociceptive effect of 5-HTP observed in this study. Previously, it was demonstrated that administration of 5-HTP caused an increase in 5-HT content in the intestine ${ }^{30}$ and a lowering of the threshold to noxious colorectal distension in rats through $5 \mathrm{HT}_{3}$ receptors. ${ }^{31}$ In line with the hypothesis on the pro-nociceptive effect of 5-HTP, Cremon et al. demonstrated in patients with IBS that mucosal release of 5-HT correlated with the intensity of perceived abdominal pain/discomfort. ${ }^{24}$

With regards to visceral perception, we observed that administration of 5-HTP resulted in increased pain perception (hyperalgesia) in hypersensitive IBS patients, but not in normosensitive patients. A similar hyperalgesic reaction, although of minor intensity, was seen in a significant number of healthy controls, none of whom were hypersensitive in the placebo condition. Also, a significant number of healthy volunteers showed lowered thresholds for pain perception after 5-HTP, indicative of an allodynic response. Pain 
thresholds of these healthy controls after 5-HTP were comparable to those seen in the hypersensitive IBS patients. These healthy volunteers may represent an 'IBS-prone' phenoptype or, more specifically, a 'hypersensitivity prone' phenotype, that only become manifestly hypersensitive when interfering with its serotonergic metabolism.

Remarkably, healthy controls and IBS patients demonstrating a pronociceptive response (hyperalgesia and allodynia) to 5-HTP also showed increased intestinal permeability, supporting the hypothesis that impaired barrier function may play an important role in visceral hypersensitivity. More interestingly, 5-HTP induced a reinforcement of the intestinal barrier function in healthy controls. This may in fact represent a protective mechanism related to increased serotonergic signaling in the mucosa to prevent penetration of potentially noxious luminal substances into the lamina propria. Luminal actors or agents such as potentially noxious food allergens can stimulate $5-\mathrm{HT}$ release from enterochromaffin cells, ${ }^{32}$ triggering reinforcement of the intestinal barrier. Previous studies have provided evidence for a role of serotonin in modulating barrier function. ${ }^{19}$, ${ }^{33} \mathrm{~A}$ potential role for serotonergic signaling in regulation of the intestinal barrier is supported by our observation that expression of ZO-1 mRNA positively correlates with mucosal 5-HIAA levels.

In IBS patients, on the contrary to healthy controls, 5-HTP administration did not result in a reinforcement of the barrier function. Even though a similar shift in the distribution of tight junction protein ZO-1 protein was seen in IBS patients as in healthy controls, as seen in Figure 1, this was not accompanied by either an increase in ZO-1 mRNA or a decrease in the sugar permeability. The inability to enhance barrier function in IBS may be related to the fact that the expression and transcription of tight junction proteins was perturbed. This may be indicative of a primary defect in the intestinal barrier, in line with other recent findings. ${ }^{7,} 34$ Failure to reinforce barrier function in response to potentially noxious stimuli, signaled through serotonin, may represent an important pathophysiological mechanism in IBS and, in particular, visceral hypersensitivity.

It appears, therefore, that the same $5-\mathrm{HT}$-mediated mechanisms aimed to reinforce the 
intestinal barrier exist in both controls and in IBS patients. However, these mechanisms malfunction in the IBS group possibly resulting from a primary defect of the intestinal tight junctions. Increased baseline 5-HT turnover in IBS could also, in fact, reflect an attempt to reinforce the intestinal barrier. However, whether alterations in serotonergic metabolism are a cause or a consequence of impaired barrier function is yet to be established.

Interestingly, the impaired intestinal barrier and the failure to reinforce the barrier following the serotonergic challenge was observed regardless whether IBS patients were hypersensitive. We therefore postulate that hypersensitivity in IBS develops as a consequence of impaired barrier function. This impairment could potentially result in an augmented viscero-sensory response to increased serotonergic signaling. Indeed, normoen hypersensitive patients both exhibit impaired barrier functions and an increased 5-HT metabolism, whereas only hypersensitive patients demonstrate a pro-nociceptive response to increased serotonergic signaling caused by oral 5-HTP administration. The increased baseline plasma levels of 5-HIAA also reflect the enhanced in serotonergic metabolism in hypersensitive IBS patients.

This study is the first to report on the effect of serotonergic modulation on intestinal permeability in IBS in relation to visceral perception. Our data contribute to a better understanding of serotonergic signaling, in particular in relation to changes in intestinal integrity and visceral hypersensitivity in the pathophysiology of IBS and may pave ways to develop novel therapeutic modalities in functional gastrointestinal disorders such as IBS. 
8.5. FIGURE LEGENDS

\section{A}

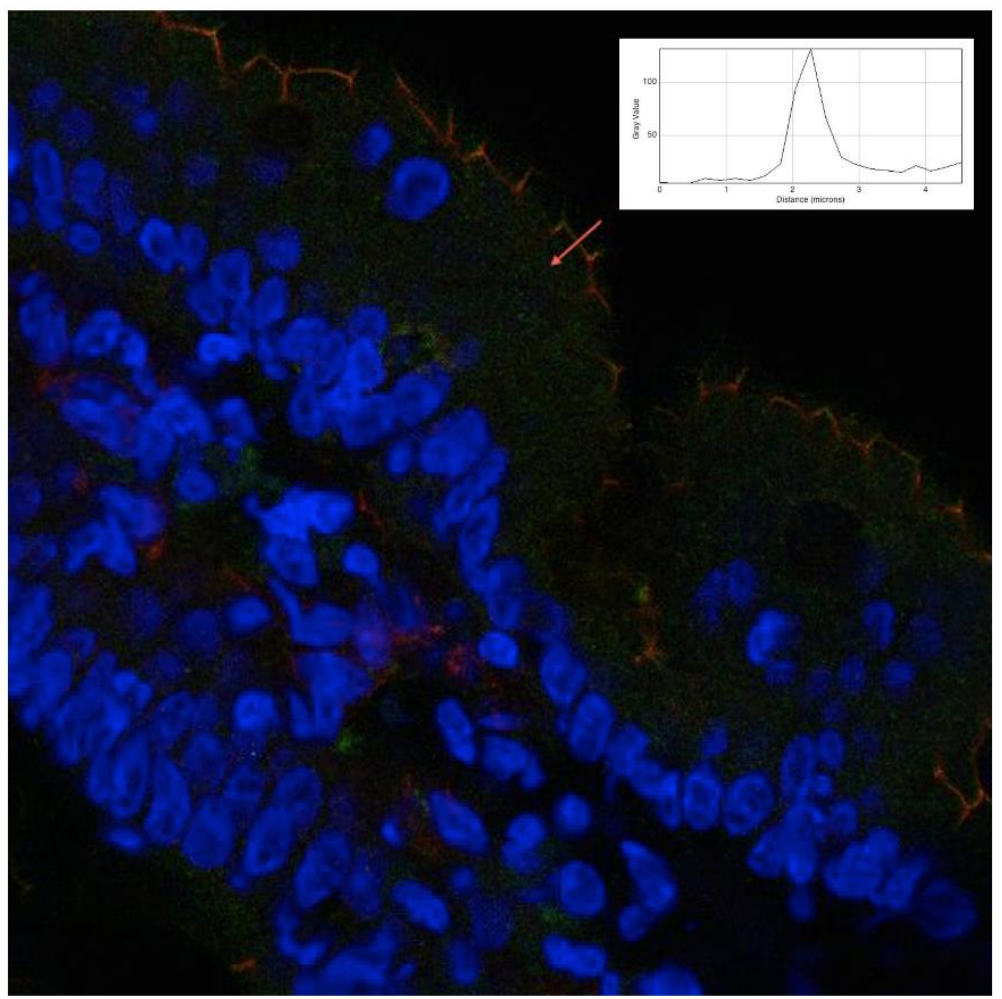

\begin{tabular}{lllllll}
\hline AUCs & IBS & IBS & Confidence & $\begin{array}{l}\text { Healthy } \\
\text { (placebo) }\end{array}$ & $\begin{array}{l}\text { Healthy } \\
\text { (5-HTP) }\end{array}$ & $\begin{array}{l}\text { Confidence } \\
\text { interval }\end{array}$ \\
& (placebo) & $\mathbf{( 5 - H T P )}$ & interval & 247.61 & 216.8 & $215.6-217.5$ \\
ZO-1 & 133.4 & 110.0 & $103.1-116.6$ & 247.6 & 134.1 & $133.9-134.3$ \\
\hline
\end{tabular}


B

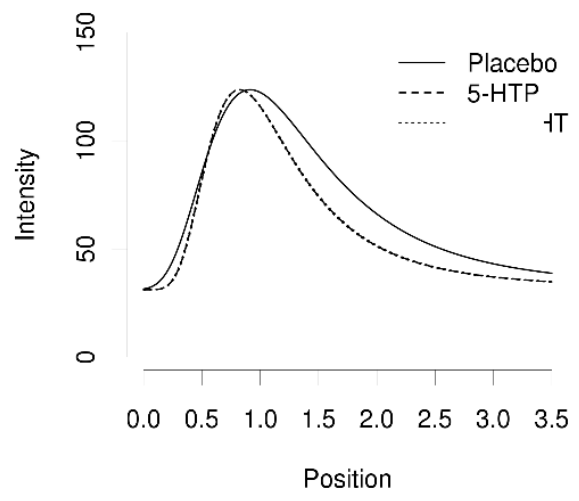

\section{Healthy Occludin}

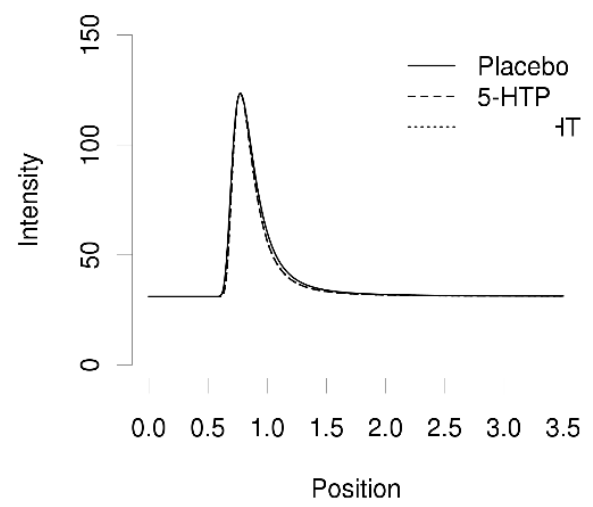

IBS ZO-1

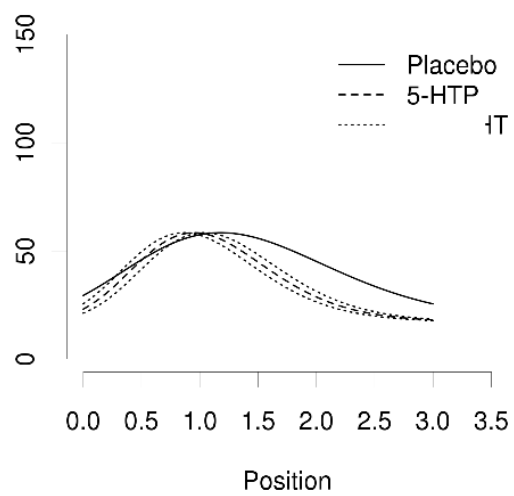

IBS Occludin

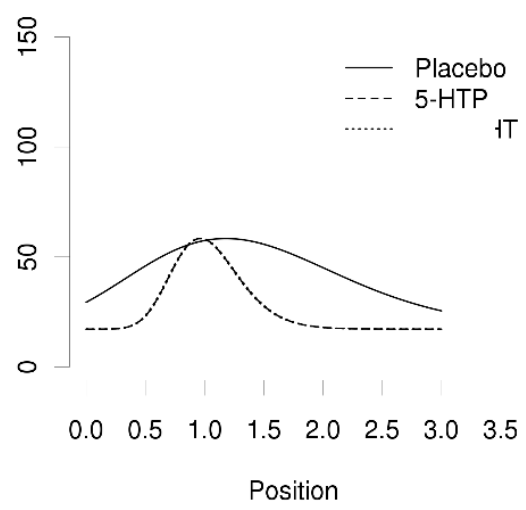

Figure 1: Immunohistochemical staining for zonula occludens-1 (ZO-1) and occludin proteins. Panel A depicts a representative microscopic image of a villus stained for tight junctions (green: ZO-1, red: occludin) and nuclei (blue). The arrow depicts the perpendicular line drawn to assess staining intensity. The insert demonstrates the generated plot profile of the staining intensity. Panel B demonstrates staining intensity is plotted against location within the cell. Zero indicates the luminal side of the cell. Lines indicate the distribution of staining intensity as a function of the cellular location in the regions investigated (apical and cytosolic). The dotted line indicates the confidence interval (Cl) of the minimal and maximal difference in staining intensity for 5-hydroxytryptopan (5HTP) as compared to the placebo condition. The table insert demonstrates corresponding values for area under the curves (AUCs). 
A

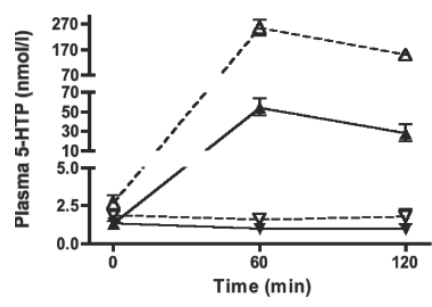

C
B

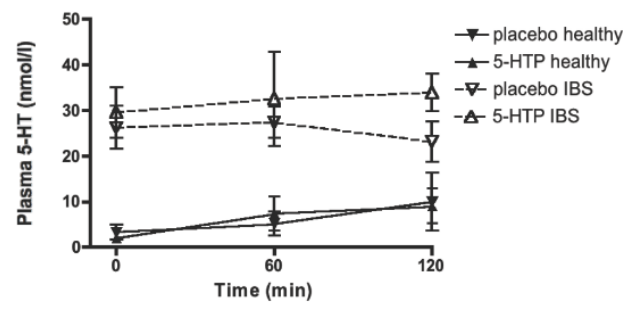

D

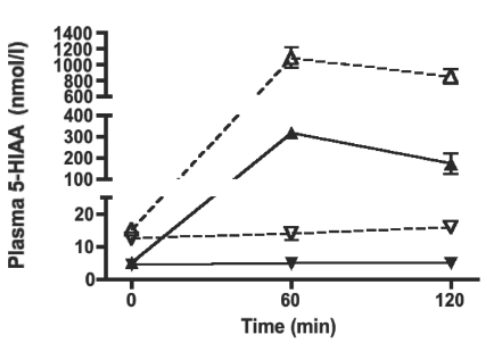

E

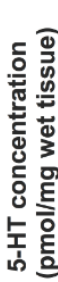

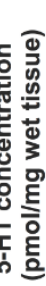
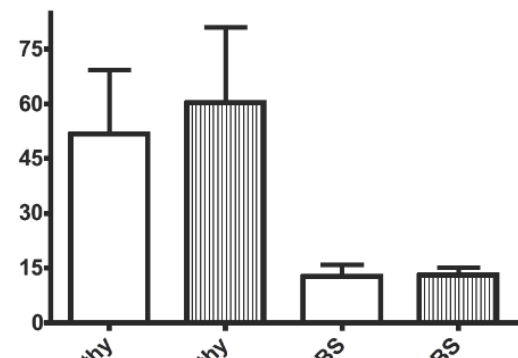

- placebo healthy

- $\boldsymbol{\nabla}$ - placebo IBS

-A- 5-HTP IBS

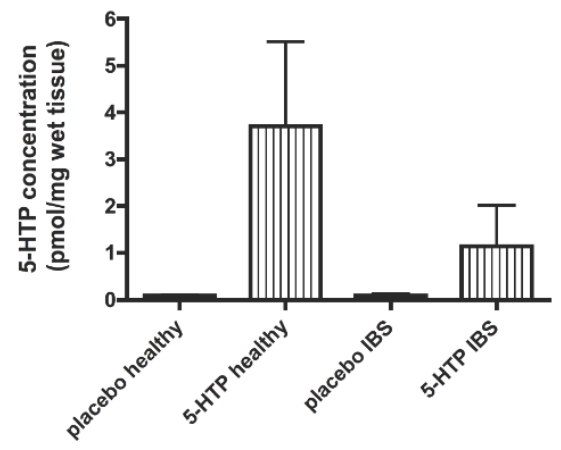

$\mathrm{F}$
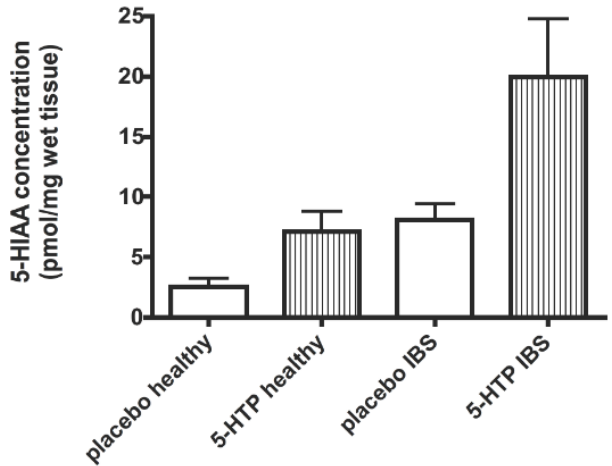

Figure 2: Concentration of serotonin metabolites in healthy controls and IBS patients as 
measured in plasma (panels A, B and C) and duodenal mucosa (panels D, E and F). Concentrations of 5-hydroxytryptophan (5-HTP), serotonin (5-HT) and 5hydroxyindoleacetic acid (5-HIAA) were measured following 5-HTP and placebo intake.

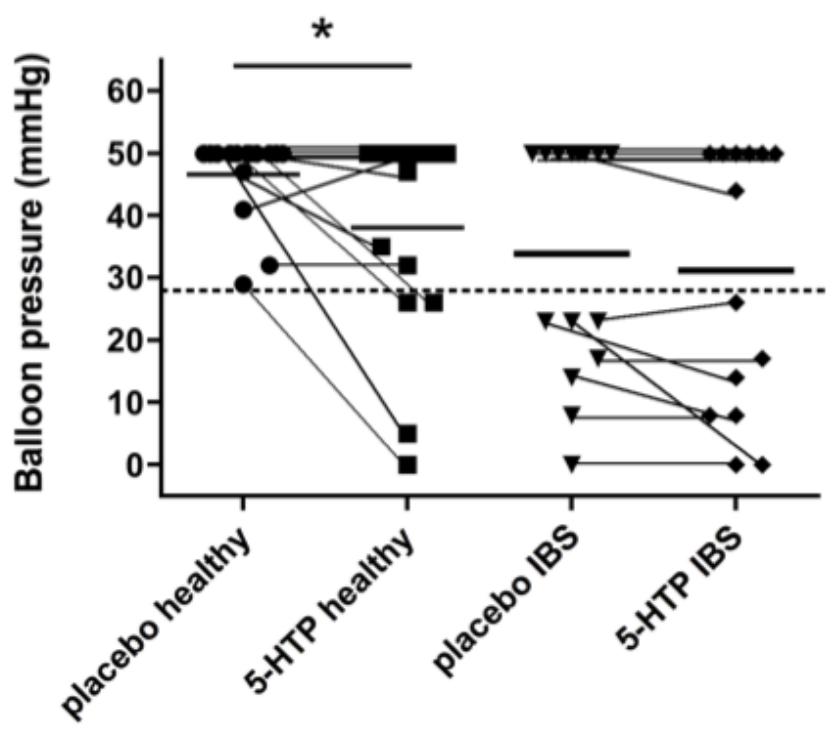

Figure 3: Pressure thresholds for pain in healthy controls and IBS patients following placebo and 5-hydroxytryptopan (5-HTP) intake. The dashed line represents the pressure level used for defining hypersensitivity (26 mmHg). ${ }^{*} \mathrm{p}<0.05$
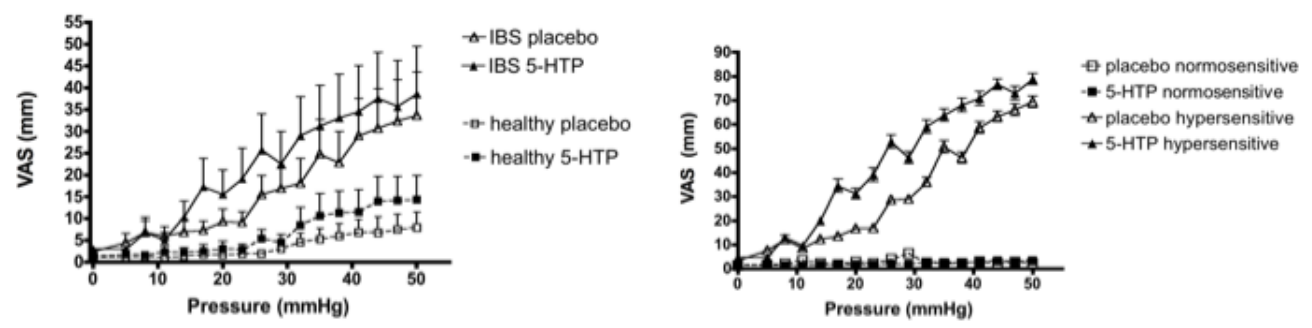

Figure 4: Visual analogue scale (VAS) scores for pain intensity during rectal distension following 5-hydroxytryptopan (5-HTP) and placebo intake in healthy controls versus IBS patients (panel A) and in normosensitive versus hypersensitive IBS patients (panel B). 


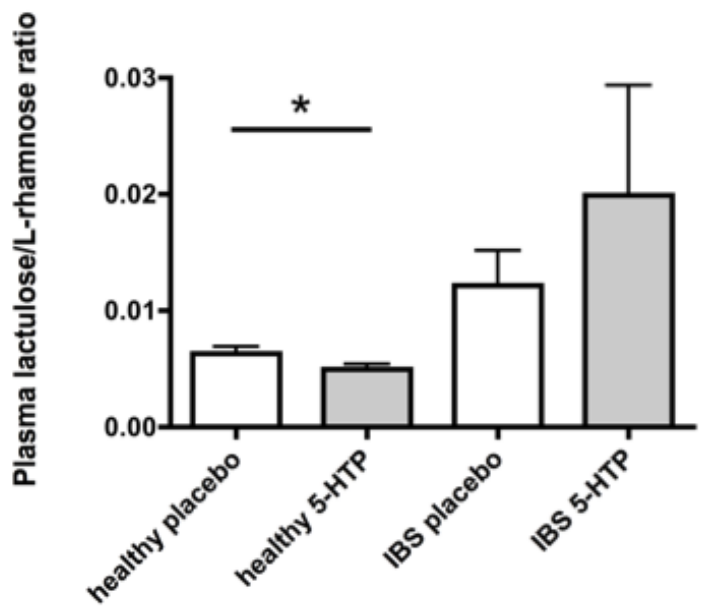

Figure 5: Plasma lactulose/rhamnose ratio in healthy controls and patients with irritable bowel syndrome (IBS) after placebo and 5-hydroxytryptopan (5-HTP). " $0<0.05$
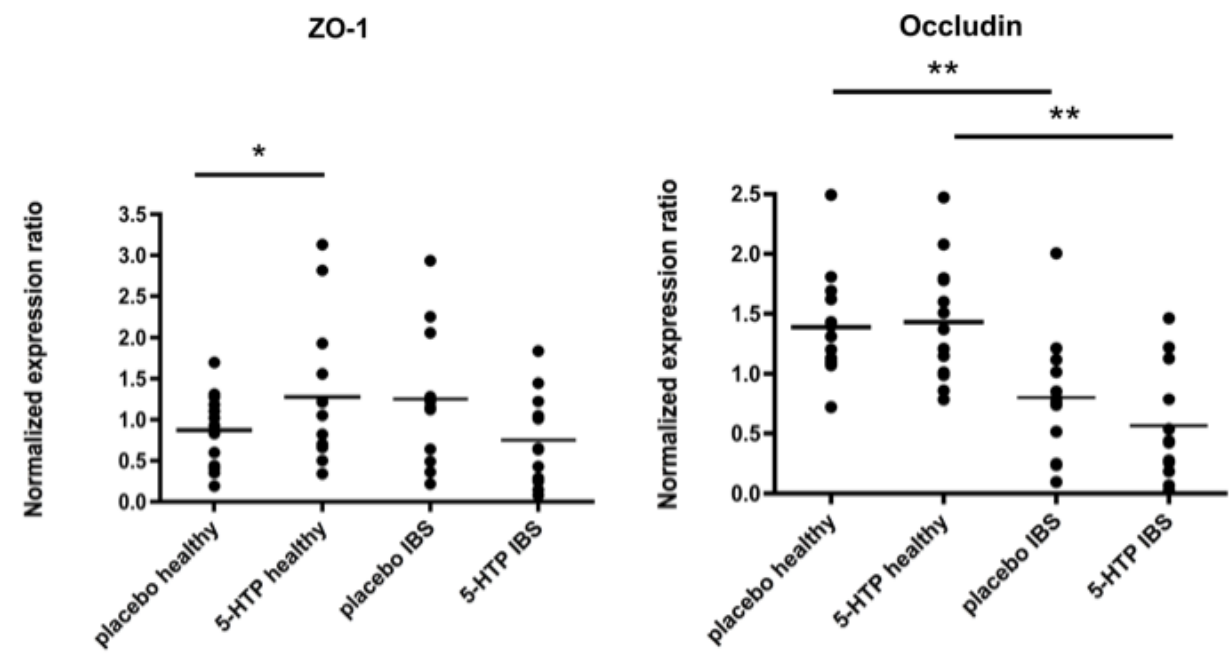

Figure 6: Transcription of tight junction proteins zonula occludens-1 (ZO-1, panel A) and occludin (panel B) in duodenal mucosal samples of healthy controls and IBS patients. mRNA concentrations were measured following 5-hydroxytryptophan (5-HTP) and placebo intake and are expressed as normalized expression ratio. ${ }^{*} \mathrm{p}<0.05,{ }^{* *} \mathrm{p}<0.01$ 


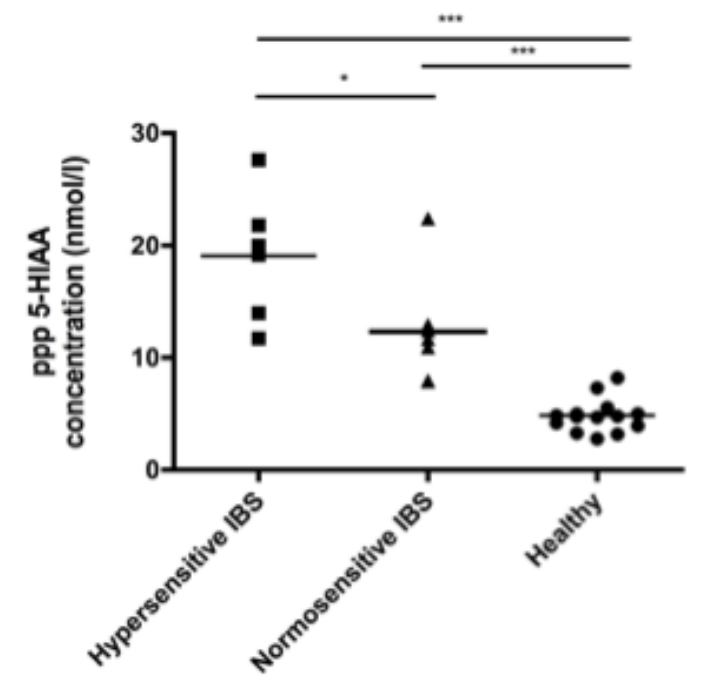

Figure 7: Platelet poor plasma (ppp) levels of 5-hydroxyindoleacetic acid (5-HIAA) following placebo intake in hypersensitive, normosensitive IBS patients and healthy controls. ${ }^{*} p<0.05 ;{ }^{* * *} p<0.001$. 


\section{6.}

\section{REFERENCES}

1. Drossman DA, Camilleri M, Mayer EA, et al. AGA technical review on irritable bowel syndrome. Gastroenterology 2002;123:2108-31.

2. Camilleri M. Serotonin in the gastrointestinal tract. Curr Opin Endocrinol Diabetes Obes 2009; 16:53-9.

3. Keszthelyi D, Troost FJ, Masclee AA. Understanding the role of tryptophan and serotonin metabolism in gastrointestinal function. Neurogastroenterol Motil 2009;21:1239-49.

4. Foley S, Garsed K, Singh G, et al. Impaired uptake of serotonin by platelets from patients with irritable bowel syndrome correlates with duodenal immune activation. Gastroenterology 2011;140:1434-43 e 1.

5. Wilder-Smith $\mathrm{CH}$. The balancing act: endogenous modulation of pain in functional gastrointestinal disorders. Gut 2011;60:1589-99.

6. Camilleri M, Lasch K, Zhou W. Irritable bowel syndrome: methods, mechanisms, and pathophysiology. The confluence of increased permeability, inflammation, and pain in irritable bowel syndrome. Am J Physiol Gastrointest Liver Physiol 2012;303:G775-85.

7. Bertiaux-Vandaele N, Youmba SB, Belmonte L, et al. The expression and the cellular distribution of the tight junction proteins are altered in irritable bowel syndrome patients with differences according to the disease subtype. Am J Gastroenterol 2011;106:2165-73.

8. Martinez C, Lobo B, Pigrau M, et al. Diarrhoea-predominant irritable bowel syndrome: an organic disorder with structural abnormalities in the jejunal epithelial barrier. Gut 2013;62(8): 1160-8.

9. Martinez C, Vicario M, Ramos L, et al. The jejunum of diarrhea-predominant irritable bowel syndrome shows molecular alterations in the tight junction signaling pathway that are associated with mucosal pathobiology and clinical manifestations. Am J Gastroenterol 2012;107:736-46.

10. Ait-Belgnaoui A, Bradesi S, Fioramonti J, et al. Acute stress-induced hypersensitivity to colonic distension depends upon increase in paracellular permeability: role of myosin light chain kinase. Pain 2005;113:141-7.

11. Zhou Q, Zhang B, Verne GN. Intestinal membrane permeability and hypersensitivity in the irritable bowel syndrome. Pain 2009;146:41-6.

12. Gijsman HJ, van Gerven JM, de Kam ML, et al. Placebo-controlled comparison of three dose-regimens of 5-hydroxytryptophan challenge test in healthy volunteers. $\mathrm{J}$ Clin Psychopharmacol 2002;22:183-9.

13. Ludidi S, Conchillo JM, Keszthelyi D, et al. Does meal ingestion enhance sensitivity of visceroperception assessment in irritable bowel syndrome? Neurogastroenterol Motil 2012;24:47-53, e3.

14. van Wijck K, Verlinden TJ, van Eijk HM, et al. Novel multi-sugar assay for sitespecific gastrointestinal permeability analysis: A randomized controlled crossover trial. Clin Nutr 2013;32(2):245-51.

15. Keszthelyi D, Troost FJ, Jonkers DM, et al. Does acute tryptophan depletion affect peripheral serotonin metabolism in the intestine? Am J Clin Nutr 2012;95:603-8. 
16. Lindsey JK. Nonlinear Models in Medical Statistics. Oxford University Press, 2001.

17. Ludidi S, Conchillo JM, Keszthelyi D, et al. Rectal hypersensitivity as hallmark for irritable bowel syndrome: defining the optimal cutoff. Neurogastroenterol Motil 2012;24:729-e346.

18. van der Veek PP, Van Rood YR, Masclee AA. Symptom severity but not psychopathology predicts visceral hypersensitivity in irritable bowel syndrome. Clin Gastroenterol Hepatol 2008;6:321-8.

19. Haub S, Kanuri G, Volynets V, et al. Serotonin reuptake transporter (SERT) plays a critical role in the onset of fructose-induced hepatic steatosis in mice. Am J Physiol Gastrointest Liver Physiol 2010;298:G335-44.

20. Keszthelyi D, Troost FJ, Simren M, et al. Revisiting concepts of visceral nociception in irritable bowel syndrome. Eur J Pain 2012;16:1444-54.

21. Magnussen I, Jensen TS, Rand JH, et al. Plasma accumulation of metabolism of orally administered single dose L-5-hydroxytryptophan in man. Acta Pharmacol Toxicol (Copenh) 1981:49:184-9.

22. Gill RK, Pant N, Saksena S, et al. Function, expression, and characterization of the serotonin transporter in the native human intestine. Am J Physiol Gastrointest Liver Physiol 2008;294:G254-62.

23. Lambert GW, Kaye DM, Cox HS, et al. Regional 5-hydroxyindoleacetic acid production in humans. Life Sci 1995;57:255-67.

24. Cremon C, Carini G, Wang B, et al. Intestinal serotonin release, sensory neuron activation, and abdominal pain in irritable bowel syndrome. Am $\mathrm{J}$ Gastroenterol 2011;106:1290-8.

25. Kerckhoffs AP, Ter Linde JJ, Akkermans LM, et al. Trypsinogen IV, serotonin transporter transcript levels and serotonin content are increased in small intestine of irritable bowel syndrome patients. Neurogastroenterol Motil 2008;20:900-7.

26. Coates MD, Mahoney CR, Linden DR, et al. Molecular defects in mucosal serotonin content and decreased serotonin reuptake transporter in ulcerative colitis and irritable bowel syndrome. Gastroenterology 2004;126:1657-64.

27. Atkinson W, Lockhart S, Whorwell PJ, et al. Altered 5-hydroxytryptamine signaling in patients with constipation- and diarrhea-predominant irritable bowel syndrome. Gastroenterology 2006; 130:34-43.

28. Wang SH, Dong L, Luo JY, et al. Decreased expression of serotonin in the jejunum and increased numbers of mast cells in the terminal ileum in patients with irritable bowel syndrome. World J Gastroenterol 2007;13:6041-7.

29. Dunlop SP, Coleman NS, Blackshaw E, et al. Abnormalities of 5-hydroxytryptamine metabolism in irritable bowel syndrome. Clin Gastroenterol Hepatol 2005;3:349-57.

30. Udenfriend $\mathrm{S}$, Titus $\mathrm{E}$, Weissbach $\mathrm{H}$, et al. Biogenesis and metabolism of 5hydroxyindole compounds. J Biol Chem 1956;219:335-44.

31. Banner SE, Sanger GJ. Differences between 5-HT3 receptor antagonists in modulation of visceral hypersensitivity. Br J Pharmacol 1995;1 14:558-62.

32. Grundy D. 5-HT system in the gut: roles in the regulation of visceral sensitivity and motor functions. Eur Rev Med Pharmacol Sci 2008;12 Suppl 1:63-7.

33. Pai VP, Horseman ND. Multiple cellular responses to serotonin contribute to epithelial homeostasis. PLoS One 2011;6:e 17028. 
34. Coeffier M, Gloro R, Boukhettala N, et al. Increased proteasome-mediated degradation of occludin in irritable bowel syndrome. Am $\mathrm{J}$ Gastroenterol 2010;105:1181-8. 
Mechanistic insight using the serotonin precursor 5-hydroxytryptophan 
Systematic review: Is microscopic colitis a drug-induced disease?

D. Keszthelyi, J. Penders, AA. Masclee, M. Pierik

Journal of Clinical Gastroenterology 2012 Nov-Dec;46(10):811-22

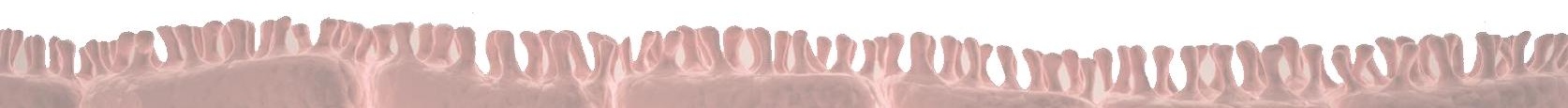




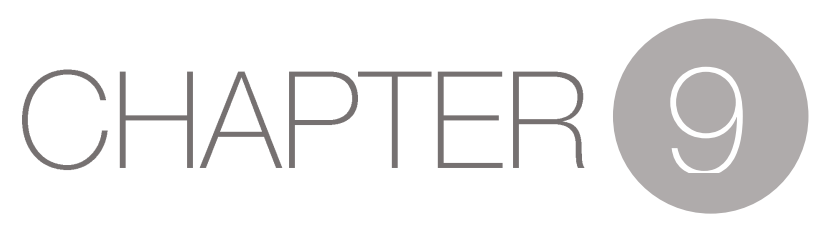

Systematic review on the role of drugs in microscopic colitis 


\section{ABSTRACT}

Microscopic colitis (MC) is the diagnosis that accounts for up to $13 \%$ of patients investigated for chronic diarrhea, particularly in middle-aged and elderly patients. Recent studies have suggested an etiological role for various drugs, including non-steroidal antiinflammatory drugs (NSAIDs) and proton pump inhibitors (PPIs). To ascertain the potential role for drug exposure in the development of $M C$, we performed a systematic review based on a MEDLINE search and conducted a meta-analyses on the available data. We also give an overview of the case reports and studies illustrating the role of drugs in inducing microscopic colitis. A number of hypotheses are formulated with regards to potential pathophysiological mechanisms in drug-induced MC. However, confirmative evidence is still largely lacking. Considering the high number of drug users and the relatively low incidence of $M C$, it is more likely that drug-induced cases of $M C$ are the result of an idiosyncratic reaction. 


\subsection{INTRODUCTION}

Microscopic colitis (MC) is an umbrella term for collagenous colitis (CC) and lymphocytic colitis (LC). MC is characterized by the combined presence of watery diarrhea, a macroscopically normal ileocolonoscopy and typical microscopic findings.' The histological changes of microscopic colitis comprise an increased number of intraepithelial CD8+ T-lymphocytes, exceeding 20 per 100 surface epithelial cells, accompanied by superficial epithelial damage and a variable inflammatory infiltrate in the lamina propria. In addition, in CC, a thickened subepithelial collagen band, which exceeds $10 \mu \mathrm{m}$, is present. These histological characteristics are not pathognomic as they may occur in various inflammatory conditions in the colon. ${ }^{2}$

MC can be diagnosed in patients of any age, but primarily affects the elderly. The average age of patients diagnosed with LC or CC ranges from 53 to 69 years. ${ }^{3}$ Also, MC appears to be more frequent in women than in men. In the past, MC was thought to be a rare disorder. However, it has recently become apparent that MC is diagnosed in up to $13 \%$ of patients investigated for chronic diarrhea, particularly if middle-aged or elderly.2, 4 Pardi et al. reported an increasing annual incidence rate up to 7.1 for collagenous colitis and up to 12.6 for lymphocytic colitis per 100,000 person-years in the period of 1998 to 2001.4 Along the same line, Scandinavian studies have also shown increasing incidences for both LC and CC (see Table 1).

\begin{tabular}{|c|c|c|c|c|}
\hline & $\begin{array}{l}\text { Period } \\
\text { investigated }\end{array}$ & $\mathrm{CC}$ & LC & Reference \\
\hline \multirow[t]{2}{*}{ USA } & $1985-1997$ & 1.6 & 2.7 & Pardi 2007 (4) \\
\hline & $1998-2001$ & 7.1 & 12.6 & \\
\hline \multirow[t]{2}{*}{ Sweden } & 1984-1993 & 1.8 & No data & Bohr $1995(73)$ \\
\hline & 1993-1998 & 4.9 & 4.4 & Olesen 2004 (8) \\
\hline \multirow{3}{*}{$\begin{array}{c}\text { Iceland } \\
\text { Spain }\end{array}$} & $1995-1998$ & 5.2 & 4.0 & Agnarsdottir 2002 (74) \\
\hline & 1993-1997 & 2.3 & 3.7 & Fernandez-Banares 1999 (75) \\
\hline & $2002-2004$ & 4.6 & 5.4 & Williams 2008 (28) \\
\hline
\end{tabular}

Table 1: Incidence rates (per 100,0000) of collagenous colitis (CC) and lymphocytic colitis 
It is apparent that the induction of inflammation in the lamina propria is a key pathogenetic factor in MC. However, the exact mechanisms involved in the initiation and development of $\mathrm{MC}$ remain to be elucidated and several pathophysiological mechanisms have been proposed. Genetic susceptibility was suggested because of reports of familial clustering of the disease. 5,6 Secondly, the reported seasonal variation in incidence indicates an infectious cause. ${ }^{7}$ Up to now, no causal enteropathogen has been identified and antibiotics are not effective in the treatment of MC. Over $40 \%$ of the patients with MC have co-existing autoimmune diseases such as celiac disease or thyroiditis pointing to potential involvement of autoimmune mechanisms. ${ }^{2}, 8$, 9 Furthermore, for CC, myofibroblast dysfunction has been described. ${ }^{10-12}$ Finally, noxious luminal substances such as drugs and smoking may trigger the chronic inflammation seen in MC and one postulated hypothesis points to increased colonic permeability in MC patients, thereby allowing luminal antigens to enter the lamina propria and elicit an immune and inflammatory reaction. ${ }^{13}$

The increasing incidence of MC may partially be explained by an increased awareness of the condition among clinicians and histopathologists. Moreover, a variety of drugs has been associated with MC and has even been suggested to induce microscopic colitis. The increased use of medications especially in older people might explain the reported increased incidence of microscopic colitis. ${ }^{3}$ To shed more light with regards to current evidence regarding the role of drug exposure in microscopic colitis and the mechanisms involved we performed a systematic review of the literature.

\subsection{MATERIALS AND METHODS}

\section{LITERATURE SEARCH}

We executed a MEDLINE search using the terms: 'microscopic colitis' or 'lymphocytic colitis' or 'collagenous colitis.' The language restrictions were English, French, German or Dutch. The initial search resulted in 529 hits. The abstract, or when available, the full 
paper was reviewed by 2 reviewers independently and papers were excluded if not reporting on drugs and microscopic colitis. For 13 hits, no abstract or paper was available and were therefore excluded. After initial review, 26 papers were found eligible for further review. Review of the references of the included papers resulted in 2 additional case reports. See Figure 1 for PRISMA flowchart.

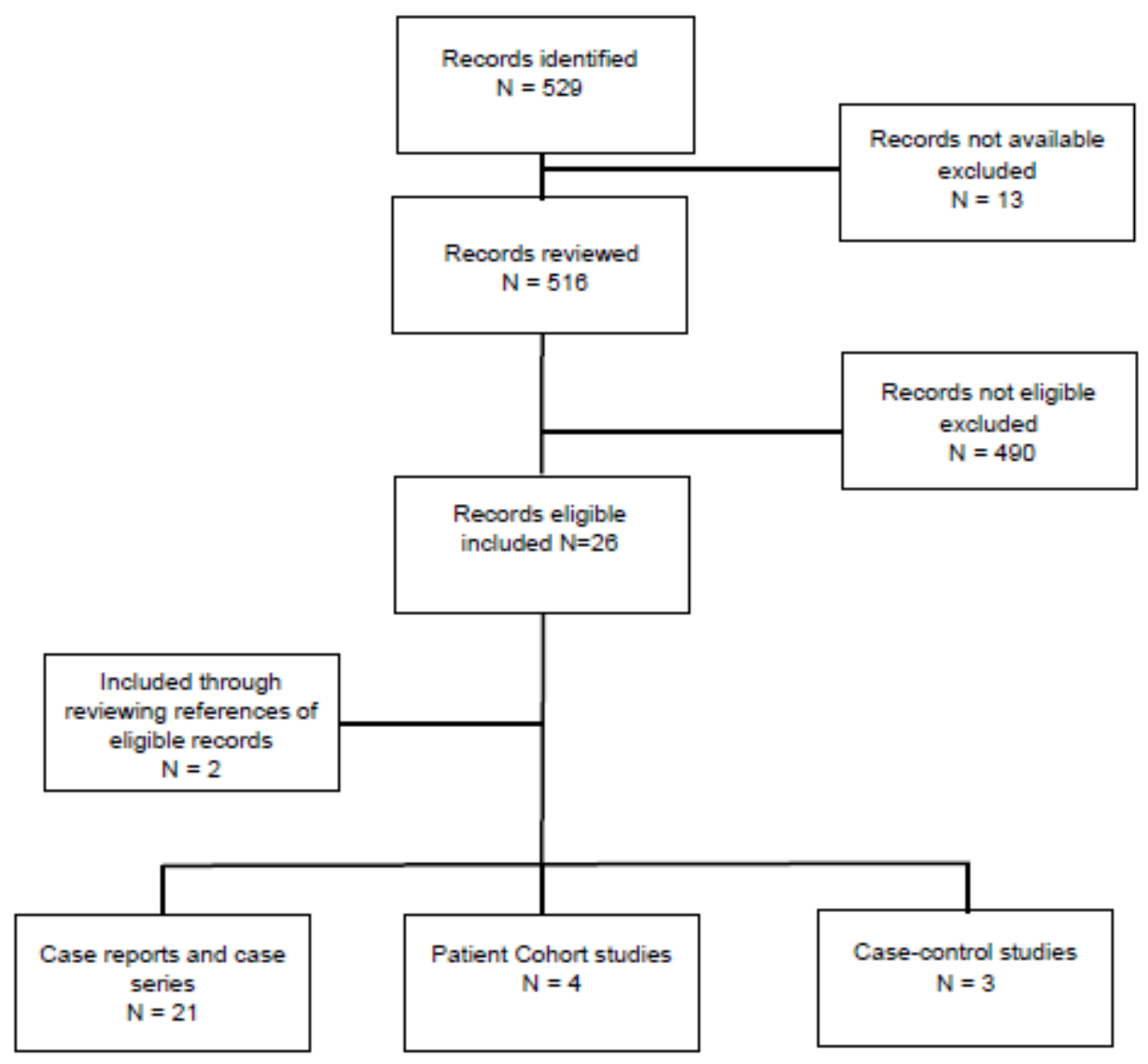

Figure 1: PRISMA flowchart for literature search.

\section{META-ANALYSIS}

Eventually, the meta-analysis was performed using data from the only three case-control studies assessing exposure to medication in microscopic colitis ${ }^{14-16}$. These three studies 


\section{Chapter 9}

all reported on non-steroidal anti-inflammatory drugs (NSAIDs) and only two of them on proton pump inhibitors (PPIs). Therefore, only data on NSAID exposure were considered for meta-analysis. Also, the patient populations examined were not entirely homogenous, some examining CC and LC separately, for instance. For this reason, in case of the study of Fernandez-Banares et al., ${ }^{15}$ we pooled the drug exposure rates from CC and LC cases prior to the meta-analysis.

For each of the included studies, Odds Ratios (ORs) and their 95\% confidence intervals were calculated based upon the reported drug exposure rates in $\mathrm{MC}$ patients and controls. To derive a pooled OR from individual studies, we used a random-effects meta-analysis model. It is important to note, that we were not able to correct ORs for age, gender and other confounders and therefore only report crude ORs. Heterogeneity was quantified with the I-square index, which describes the proportion of total variation in study estimates due to heterogeneity. ${ }^{17}$

Statistical analyses were conducted using the "metan" command within Stata version 11 (STATA Corp, College Station, Texas, USA). The results are displayed in a forest plot.

\subsection{RESULTS AND DISCUSSION}

\section{NSAIDS}

Of all drug groups suggested to play an etiological role in microscopic colitis, the most convincing evidence exists for NSAIDs. A summary of case reports of NSAID-induced microscopic colitis is given in table 2. We also give here a summary of cases reporting on the association with the exposure to Cyclo 3 forte, a venotonic drug used in France, also containing aspirin (see Table ). It is, however, important to acknowledge that it is unclear whether the association is related to aspirin or the other components on Cyclo 3 forte. 


\begin{tabular}{|c|c|c|c|c|c|c|}
\hline Treatment & 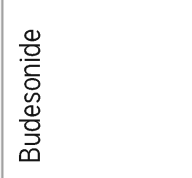 & 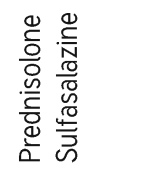 & 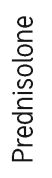 & 1 & 1 & \\
\hline No re-occurrence of diarrhea > $18 \mathrm{~m}$ & ஜ & $\bar{c}$ & , & 오 & 우 & \\
\hline Time to disappearance diarrhea & I & 로 & 호 & 모 & 호 & \\
\hline Time to re-appearance diarrhea & i & ? & gृ & ge & $\frac{3}{4}$ & \\
\hline Rechallenge & + & 옫 & 묻 & 혿 & 粋 & \\
\hline Histologic normalization & ఫृ & gृ & gृ & + & 후 & \\
\hline Time disappearance diarrhea & 옫 & 옫 & $\overline{\underline{c}}$ & 혿 & 혿 & \\
\hline Dechallenge & + & , & $\bar{c}$ & + & + & \\
\hline Time start drug to start diarrhea & D্ল & 옫 & ह్ & 옫 & 3 & \\
\hline Co-morbidities & 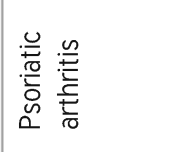 & 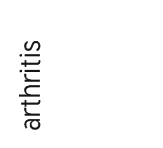 & $\begin{array}{l}\frac{\omega}{\underline{\underline{\underline{E}}}} \\
\dot{\underline{\underline{0}}} \\
\dot{\vec{U}}\end{array}$ & 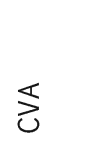 & 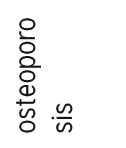 & \\
\hline Other Drugs & 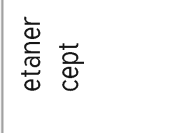 & 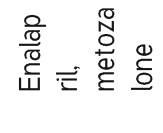 & $\mathrm{q}$ & २ & $\frac{5}{\frac{5}{\frac{5}{0}}}$ & 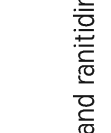 \\
\hline Gender & 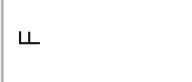 & $\Sigma$ & $\Sigma$ & 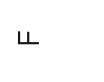 & 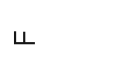 & $\begin{array}{l}. \overline{0} \\
\overline{0} \\
.0\end{array}$ \\
\hline Age & $\bar{\gamma}$ & 임 & 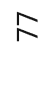 & & $\infty$ & 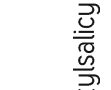 \\
\hline$\angle C$ or CC & $\breve{s}$ & u & u & U & u & $\begin{array}{l}\tilde{U} \\
\tilde{0} \\
\tilde{\Phi}\end{array}$ \\
\hline Drug & 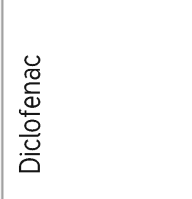 & 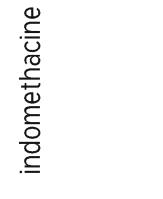 & 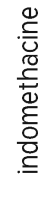 & 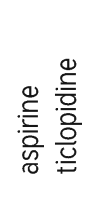 & 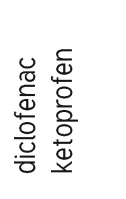 & 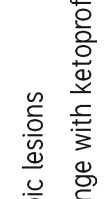 \\
\hline study & 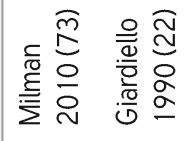 & $\begin{array}{l}\bar{\Phi} \\
\stackrel{8}{0} \\
\text { J }\end{array}$ & $\begin{array}{l}\text { N } \\
\text { 岁 } \\
\text { 心 }\end{array}$ & 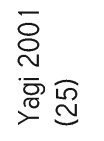 & 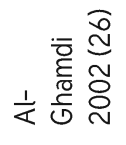 & 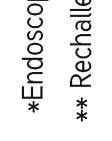 \\
\hline
\end{tabular}




\section{Chapter 9}

Treatment

No re-occurrence of diarrhea $>18 \mathrm{~m}$

Time to disappearance diarrhea

Time to re-appearance diarrhea

Rechallenge

Histologic normalization

Time disappearance diarrhea

Dechallenge

Time start drug to start diarrhea

Co-morbidities

Other Drugs

Gender

Age

LC or CC

Drug

study

\begin{tabular}{|c|c|c|c|c|c|}
\hline I & & 1 & 1 & I & 1 \\
\hline 무 & & 두 & 으 & 무 & + \\
\hline 오 & & 오 & 읃 & 모 & 으 \\
\hline 므 & & 으 & 읃 & 무 & 므 \\
\hline 모 & & 혼 & 으 & 모 & 모 \\
\hline 모 & & 모 & 모 & 모 & + \\
\hline D্ & & 모 & 모 & 모 & 모 \\
\hline+ & & 모 & 믇 & 므 & + \\
\hline$\underset{4}{3}$ & & 모 & 으 & 모 & $\stackrel{\Sigma}{\sim}$ \\
\hline $\begin{array}{l}\frac{0}{0} \\
\frac{0}{0} \\
\frac{0}{0}\end{array}$ & & 오 & 읃 & 므 & 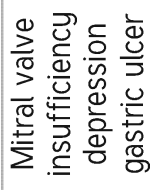 \\
\hline 1 & & 으 & 믇 & 믇 & 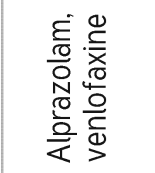 \\
\hline レ & & レ & Ш & レ & $\Sigma$ \\
\hline$\hat{m}$ & & 무 & 을 & 흘 & চ \\
\hline 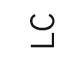 & & $\cup$ & 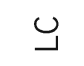 & U & U \\
\hline m & & m & 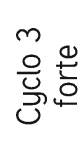 & 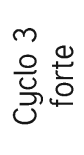 & 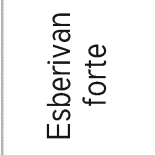 \\
\hline 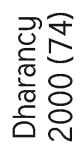 & 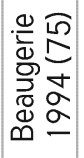 & $\begin{array}{l}-\bar{d} \\
心 \\
0 \\
0\end{array}$ & $\begin{array}{l}\sim \\
\mathbb{\Delta} \\
\ddot{U} \\
\tilde{U}\end{array}$ & $\begin{array}{l}m \\
\mathbb{D} \\
心 \\
\tilde{J}\end{array}$ & 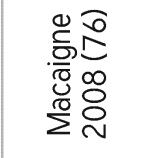 \\
\hline
\end{tabular}


Systematic review on the role of drugs in microscopic colitis

\begin{tabular}{|c|c|c|c|c|}
\hline & \multicolumn{4}{|c|}{ Study } \\
\hline & $\begin{array}{l}\text { Sveinsson } \\
2008(77)\end{array}$ & Pardi 2002 (78) & Goff 1997 (20) & Bohr 1996 (23) \\
\hline Study & Nation-wide & Single center & Single center & Registry 25 \\
\hline design & $\begin{array}{lr}\text { cohort } & \text { new } \\
\text { diagnosed } & \mathrm{MC} \\
\text { patients } & \end{array}$ & Mayo Clinic & $\begin{array}{l}\text { University } \\
\text { Michigan }\end{array}$ & Swedish hospitals \\
\hline $\begin{array}{l}\text { Inclusion } \\
\text { period }\end{array}$ & 1995-1999 & 1997-1999 & Before 1992 & 1989-1995 \\
\hline$M C$ & 125 & 189 & 31 & 163 \\
\hline$L C$ & 71 & 176 & - & - \\
\hline$C C$ & 54 & 6 & 31 & 163 \\
\hline Method & $\begin{array}{l}\text { Patient } \\
\text { Questionnaire }\end{array}$ & $\begin{array}{l}\text { Retrospective } \\
\text { chart review }\end{array}$ & $\begin{array}{l}\text { Retrospective } \\
\text { chart review and } \\
\text { Telephone } \\
\text { interview }\end{array}$ & $\begin{array}{l}\text { Retrospective } \\
\text { Chart review }\end{array}$ \\
\hline
\end{tabular}

NSAIDs at diagnosis

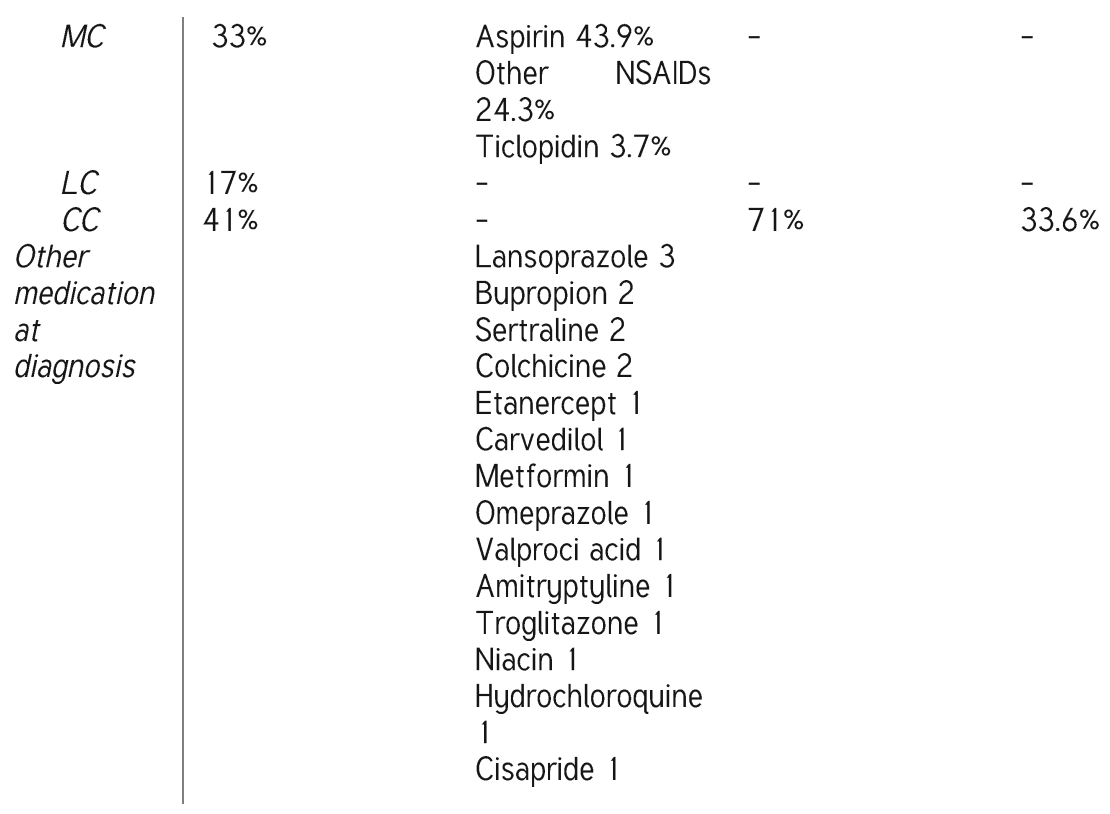

Table 4: Cohort studies assessing medication use in microscopic colitis.

* for 2 patients on Ticlopidin dechallenge resulted in disappearance of diarrhea

In an early prospective study, NSAIDs were implicated in 10\% of newly diagnosed cases of colonic inflammation, and patients taking NSAIDs had a 5-fold risk for developing a 
colonic disease..$^{18}$ In later years, a substantial number of studies have reported on a positive association with NSAID exposure and microscopic colitis ${ }^{16,19-22}$ (see Table 4 for cohort studies). This association seems more apparent with CC. ${ }^{15}$ Numerous studies have reported high use of NSAIDs among MC patients. In a retrospective review of 163 patients in Sweden with collagenous colitis, 34\% were taking NSAIDs on a regular or sporadic basis. ${ }^{23}$ Another Scandinavian study showed that $35 \%$ of the 104 patients with MC examined used NSAIDs. ${ }^{24}$ In a case control study by Riddell, 31 patients with collagenous colitis were compared to 31 controls with irritable bowel syndrome or diverticulosis. Longterm use of NSAIDs (for more than 6 months) was reported in 19 of 31 subjects with microscopic colitis, compared to only 4 of 31 controls. The case for an etiological role for NSAID use and microscopic colitis was strengthened by reports of clinical and histologic improvement among NSAID users when the medication was discontinued. $16,22,25,26$ In the controlled study of 31 patients with collagenous colitis described previously, three subjects reported reduction in diarrhea after they stopped taking NSAIDs. One subject, who later resumed taking an NSAID, developed recurrent diarrhea that resolved when the NSAID was again discontinued. ${ }^{16}$

Given this apparent role for NSAIDs in MC, we performed a meta-analyses based on the three case-controls studies published to date (pooled OR 3.25 [1.1-9.5], see Figure 2 for forest plot). Although we did calculate the pooled OR for NSAID exposure, it is important to note that this should be interpreted with caution for the following reasons. First, the number of published studies available for analysis is limited. Second, no correction was possible for other confounders, since the Riddell et al. ${ }^{16}$ did not present adjusted analyses, while we had to pool the drug exposure rates of $L C$ and CC for a the study by FernandezBanares et al. ${ }^{15}$ prior to meta-analyses. Third, the studies report on different patients populations (MC pooled together or LC and CC separately) as well as control population (general population, patients controls or a mixture of both). We therefore consider the findings of this meta-analyses indicative rather than conclusive regarding a possible etiological role for NSAIDs. 
NSAID use and Microscopic Colitis

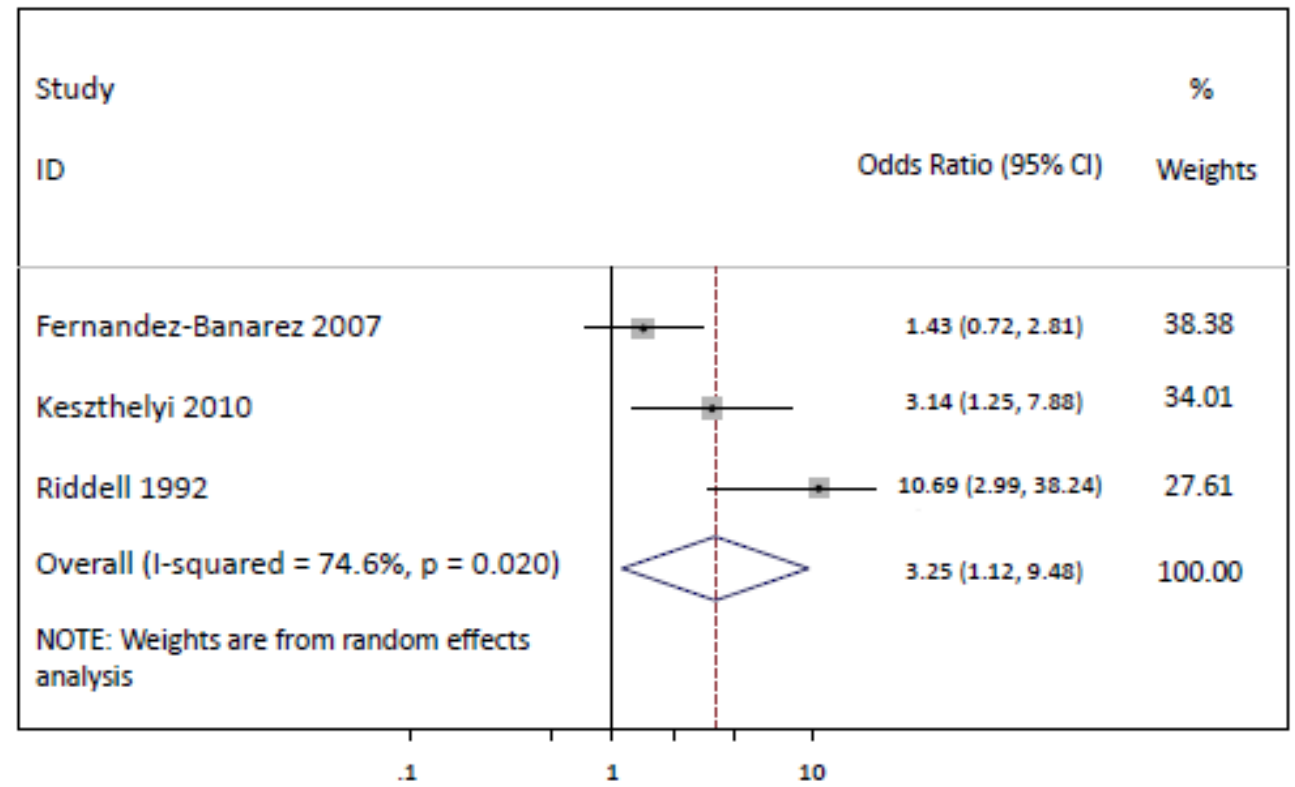

Figure 2: Forest plots for meta-analysis of non-steroidal anti-inflammatory drug (NSAID) use in microscopic colitis.

A potential confounding factor is the presence of coexisting arthralgia in patients with MC, which could increase their use of NSAIDs. ${ }^{27}$ A study reported that 18 of 31 (56\%) patients with collagenous colitis had some form of arthritis; and $71 \%$ of those were using NSAIDs regularly at the time of diagnosis. ${ }^{28}$ To establish here a cause-effect relationship is exceptionally challenging and cannot be performed based on the currently available datasets. Nevertheless, several hypotheses can be postulated with regard to potential pathophysiological mechanisms. Intestinal side effects of NSAID have been documented extensively. NSAIDs induce small intestinal and colonic injury and inflammation and possibly may exacerbate IBD. ${ }^{21}$ Furthermore, $60-70 \%$ of patients on long-term NSAID may have asymptomatic enteropathy. ${ }^{21}$ NSAID intake has in fact been associated with increased risk for acute diarrhea. ${ }^{29}$ More recently, Gleeson et al. reported that $74 \%$ of new cases presenting with colitis had been using NSAIDs prior to the development of their disease. ${ }^{30}$

Therefore, the alleged ability of NSAIDs to cause or at least exacerbate microscopic colitis 
is not altogether unexpected. NSAID-related erosions and ulcers are most commonly seen in the distal ileum and rectum. Small intestinal injury due to NSAIDs differs pathophysiologically from that found in the stomach, as prostaglandin suppression does not play an important role. ${ }^{31}$ Rather, NSAIDs and bile have synergistic effects with respect to injury of the small intestine by contact irritation of the mucosa. The enterohepatic circulation appears to be crucial in this regard, by allowing repeated exposure to the offending chemicals. In the small intestine, NSAIDs uncouple mitochondrial oxidative phosphorylation leading to reduced intracellular ATP levels. This, in turn, leads to loss of cytoskeletal control over tight junctions and increased paracellular permeability. ${ }^{32}$ This increased permeability may allow passage of certain, yet to be identified luminal antigens, which can elicit a malicious immune response, which, provided such mechanism is also present in the colon, may potentially result in clinically manifest microscopic colitis.

\section{PPIS}

Recent studies have emphasized the association between exposure to PPIs and occurrence of MC. Many case reports have been published which further highlight the association between PPI use and development of $M C^{33-36}$ see Table 5. Initially, lansoprazole has been associated with a high likelihood of inducing MC. ${ }^{37}$ In most published cases, symptoms started within few weeks after start of lansoprazole therapy (median 21.5 days), and complete resolution after lansoprazole discontinuation was observed within a few days (median 7 days), without need for further therapy. ${ }^{38}$ Another recent study also pointed to similar effects in case of exposure to omeprazole and esomeprazole. ${ }^{36}$ PPIs are one of the most frequently prescribed classes of medications worldwide because they combine a high level of efficacy with low toxicity. In 2006, expenditure on these drugs was 10 billion USD globally. ${ }^{39}$ In the five years since the introduction of esomeprazole in 2001, prescriptions for PPIs have doubled. ${ }^{39}$ It is noteworthy, that the increasing incidence of $\mathrm{MC}^{4}$ seems to parallel the rising use of PPIs.

Besides a number of case reports and case series, only a very limited number of controlled studies have examined the role of PPI exposure in MC, ${ }^{14,15}$ of which only one found a 
positive association. ${ }^{14}$ More comprehensive studies are still lacking for PPIs to support an etiological role for PPIs in MC.

The potential pathophysiological mechanisms underlying PPI-related induction of MC are poorly understood. When patients are prescribed PPIs to treat upper gastrointestinal disorders, it is important to recognize that PPIs are interacting at multiple targets. Proton pumps $\left(\mathrm{H}^{+} / \mathrm{K}^{+}\right.$ATPases) are present not only on gastric epithelium but also on colonic epithelium where they contribute to whole body potassium homeostasis. ${ }^{40}$ Inhibition of the colonic proton pumps may therefore affect local electrolyte balance and compromise fluid acidification, which can possibly affect immune reactions in the colonic mucosa. Autoradiographic studies using ${ }^{3} \mathrm{H}$-lansoprazole in animal models have shown uptake of the drug in the colonic mucosa, both in upper colonic epithelial cells as well as in inflammatory cells. ${ }^{41}$

Proteins other than $\mathrm{H}^{+} / \mathrm{K}^{+}$ATPase have also been reported as targets for PPIs. Omeprazole and lansoprazole have been observed to induce smooth muscle relaxation and to inhibit contractile activity. ${ }^{40}$ This effect on contractile systems may also affect tight junction functionality since tight junction proteins are directly linked to the actinomyosin cytoskeleton. Therefore, conformational changes in the cytoskeleton of epithelial cells may result in alterations in the function of the tight junction, which leads to increased paracellular permeability. As a result, luminal contents can more easily penetrate the lamina propria causing an immune and/or inflammatory reaction. Esomeprazole has been shown to increase paracellular permeability in the upper gastrointestinal tract in vitro ${ }^{42}$ and in vivo in humans. ${ }^{43}$ Increased paracellular permeability has been observed in $\mathrm{MC}$ and is considered a key component in the induction of diarrhea. ${ }^{44}$ Accordingly, expression of the tight junction proteins occludin, claudin-4 and zonula occludens-1 (ZO-1) was found to be decreased in mucosal biopsy specimens from MC patients. ${ }^{44,45}$ We speculate that a direct or indirect effect of PPIs on colonic tight junctions and paracellular permeability, similarly to that of NSAIDs, may be a potential mechanism in the development of MC. 


\begin{tabular}{|c|c|c|c|c|c|c|c|c|}
\hline Treatment & ' & ' & & ' & 1 & 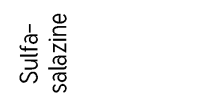 & ' & ' \\
\hline $\begin{array}{l}\text { No re-occurrence of } \\
\text { diarrhea > } 18 \mathrm{~m}\end{array}$ & ह్ & 후 & & 호 & $\bar{c}$ & gृ & $\bar{c}$ & $\bar{c}$ \\
\hline $\begin{array}{l}\text { Time to disappearance } \\
\text { diarrhea }\end{array}$ & 욷 & 훋 & & 몸 & g & 욷 & 후다 & 홍 \\
\hline $\begin{array}{l}\text { Time to re-appearance } \\
\text { diarrhea }\end{array}$ & 욷 & 후 & & 혿 & 혿 & হृ & $\underline{3}$ & 호 \\
\hline Rechallenge & 후 & 후 & & 혿 & 혿 & হृ & + & 핟 \\
\hline Histologic normalization & + & + & & + & + & 후 & + & 하 \\
\hline $\begin{array}{l}\text { Time disappearance } \\
\text { diarrhea }\end{array}$ & $\bar{x}$ & ్ㅗ & & ’ & 3 & 일 & $\bar{c}$ & $\stackrel{3}{N}$ \\
\hline Dechallenge & + & + & & + & + & + & + & + \\
\hline $\begin{array}{l}\text { Time start drug to start } \\
\text { diarrhea }\end{array}$ & 3 & ૩े & & 3 & ’ & 3 & $\bar{x}$ & ह్ \\
\hline Co-morbidities & 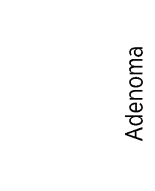 & 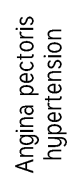 & & 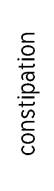 & 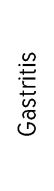 & 岧 & 苞 & 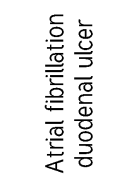 \\
\hline Other Drugs & 1 & 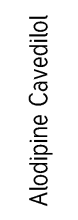 & & 호 & 호 & ' & 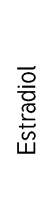 & 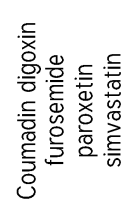 \\
\hline Gender & $\Sigma$ & $\Sigma$ & & \llcorner & \llcorner & $\Sigma$ & \llcorner & $\Sigma$ \\
\hline Age & 8 & 吕 & & $\stackrel{\infty}{\sim}$ & ڤ̊ & 우 & $\bar{n}$ & œ \\
\hline$\angle C$ or $C C$ & 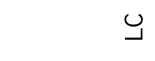 & 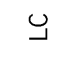 & & $\dot{u}$ & U & $\bigcup^{*} *$ & U & $\cup$ \\
\hline Drug & 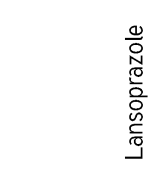 & 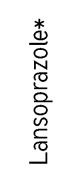 & & 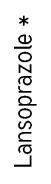 & 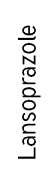 & 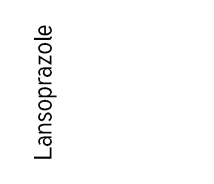 & 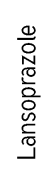 & 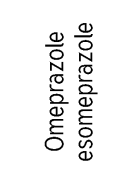 \\
\hline study & 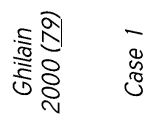 & $\begin{array}{l}\sim \\
\ddot{8} \\
\delta\end{array}$ & 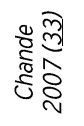 & - & 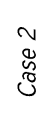 & 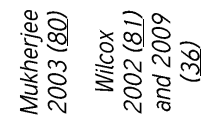 & $\bar{\Phi}$ & 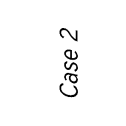 \\
\hline
\end{tabular}




\begin{tabular}{|c|c|c|c|c|c|c|c|}
\hline+ & + & + & 후 & $\bar{c}$ & $\bar{c}$ & $\bar{c}$ & $\bar{c}$ \\
\hline 든 & I & q & 무 & g & i & q & 후 \\
\hline 호 & $\underline{3}$ & E్ & 후 & $\bar{c}$ & হ & 호 & $\bar{c}$ \\
\hline g & + & + & 음 & $\bar{q}$ & 모 & 음 & 후 \\
\hline+ & + & 호 & + & + & + & + & + \\
\hline 혿 & 함 & 호 & 맞 & $\bar{x}_{x}$ & $\underline{\underline{O}}$ & 후 & D \\
\hline+ & + & + & + & + & + & + & + \\
\hline$\vec{\curvearrowleft}$ & $\vec{f}$ & $\supseteq$ & $\underline{\xi}$ & $\bar{c}$ & 흉 & $\bar{x}$ & $\underline{z}$ \\
\hline
\end{tabular}
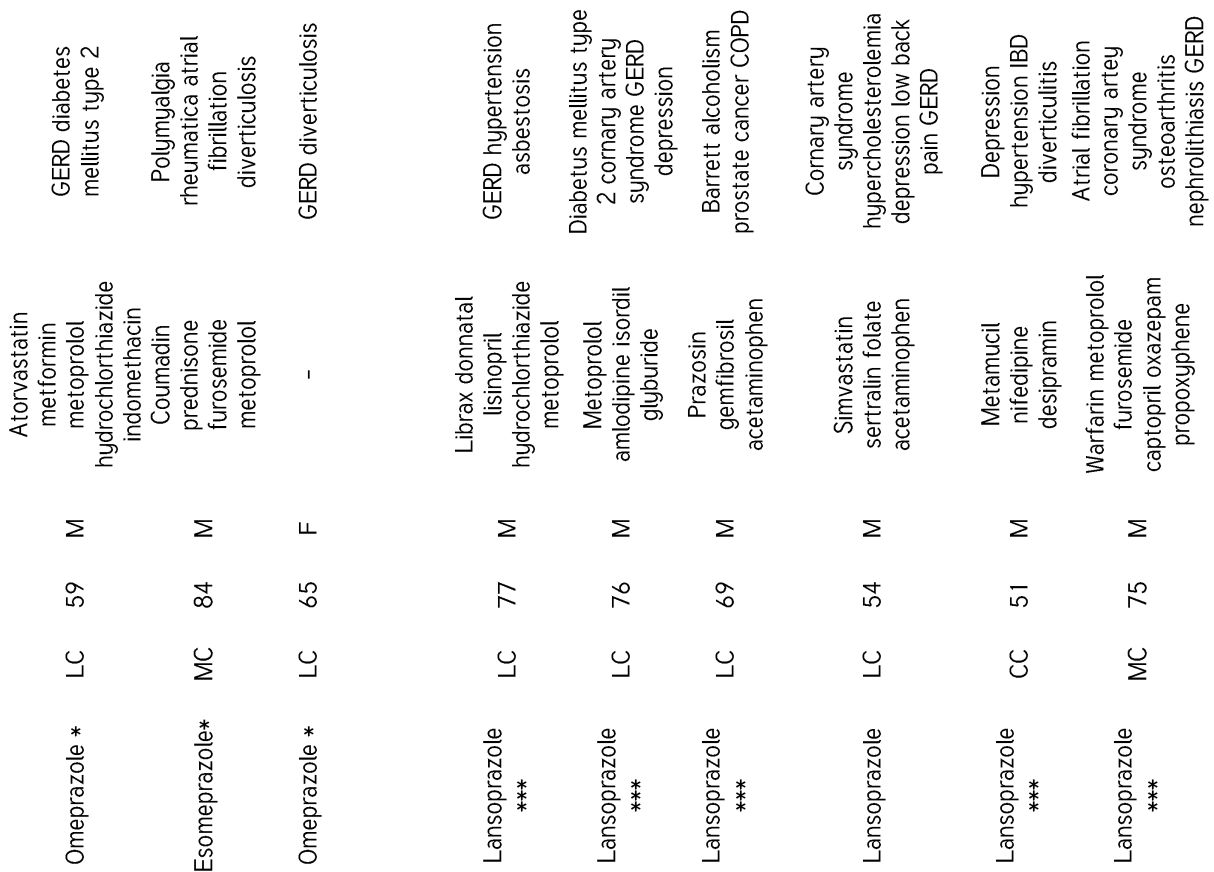

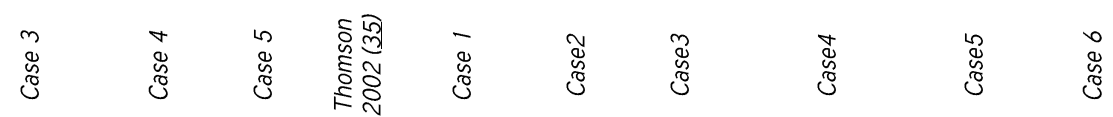




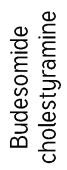

\begin{tabular}{|c|c|c|}
\hline ¿ & 호 & q \\
\hline 무 & 무 & g \\
\hline 읃 & 흗 & 등 \\
\hline ¿ & $\bar{c}$ & 후 \\
\hline+ & $\bar{c}$ & $\bar{c}$ \\
\hline 욷 & రृ & हु \\
\hline
\end{tabular}

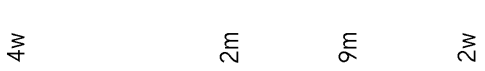

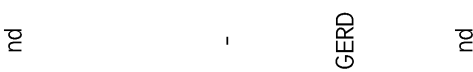

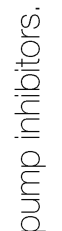

호
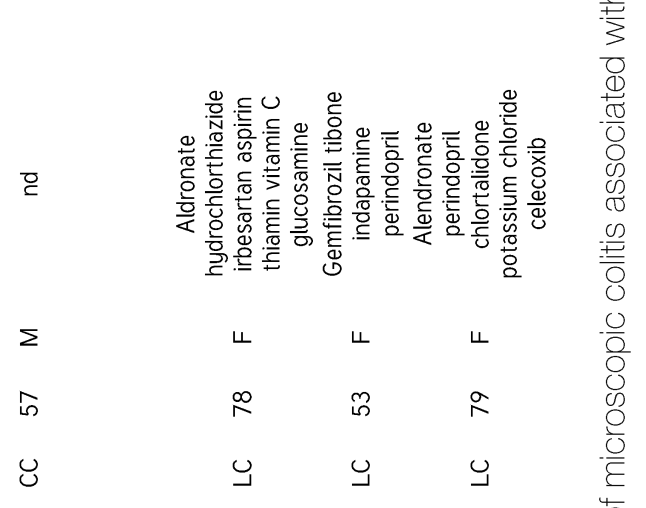

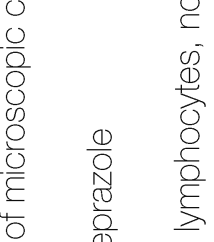
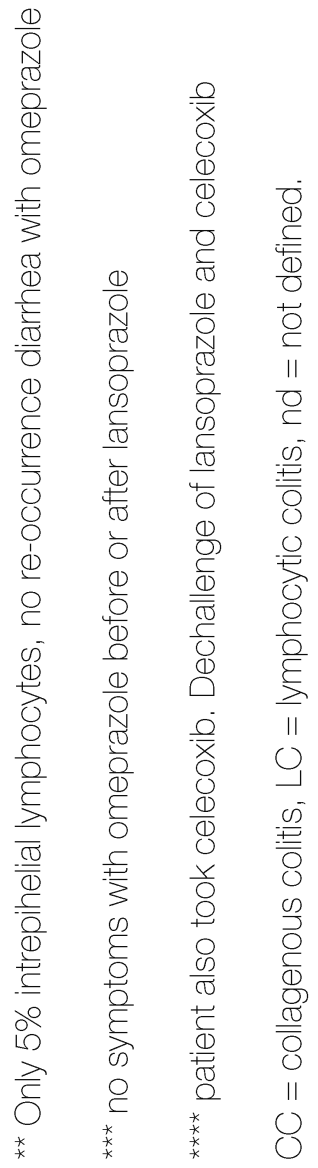
Recent case reports have related the occurrence of severe hypomagnesemia to long-time PPI exposure.46, 47 This effect has been described for omeprazole, esomeprazole, pantoprazole and lansoprazole. ${ }^{46}$ It has been proposed that PPIs induce a defect in the active absorption of magnesium in the intestine. This may result from an effect of PPIs on the tight junction proteins or on the TRPM6 and 7 channels (transient receptor potential melastin 6 and 7). The latter are key molecules involved in active magnesium absorption. Either changes in intestinal pH induced by PPIs may affect channel functions, or subjects who are heterozygous carriers of TRPM6/7 mutations are more susceptible to developing adverse reactions to PPIs. ${ }^{47}$ Presently, we cannot exclude that effects of PPIs on magnesium absorption have a role in the pathogenesis of MC. The fact that distinct genetic profiles can be of superior importance with regards to the development of side effects during PPI therapy is further supported by reports describing agranulocytosis and neutropenia induced by $\mathrm{PPI} .{ }^{48} \mathrm{~A}$ recent report demonstrated that this is caused by a mutation of the CYP2C19*17, the enzyme responsible for PPI metabolism. 49

In addition, it is well known that PPI therapy affects intestinal microbial profiles.50-53 Several bacteria, including $H$. pylori and $S$. pneumoniae, as well as fungi such as $C$. albicans, contain $\mathrm{H}^{+} / \mathrm{K}^{+}$ATPase in their plasma membranes which are highly homologous to their human counterparts. ${ }^{54}$ PPIs can therefore directly influence microbial growth by inhibition of the $\mathrm{H}^{+} / \mathrm{K}^{+}$ATPase. On the other hand, increase of intestinal $\mathrm{pH}$ can result in a diminished host defense against certain bacteria. Profound acid suppression increases the risk of enteric infections in susceptible individuals caused by Shigella, Salmonella, Yersinia or Clostridium difficile. 55 The use of PPIs may also promote the expansion and colonization of $C$. difficile by its recognized potential to induce small bowel bacterial overgrowth with anaerobic colonic organisms. ${ }^{50}$ Many clinicians believe that PPI use may therefore directly contribute to $C$. difficile and other bacterial infections. The FDA has in fact recently issued a safety announcement that PPI use may be associated with $C$. difficile infection. ${ }^{56} \mathrm{~A}$ recent study by Lombardo et al. also suggested that PPI therapy in humans may potentially result in small intestinal bacterial overgrowth (SIBO). ${ }^{57}$ Whether 


\section{Chapter 9}

these changes in intestinal microbiota induced by PPI therapy are truly responsible for the development of symptoms and clinical conditions remains subject to considerable discussion. ${ }^{58}$ Further research is needed to clarify the exact effects of PPIs on human intestinal microbiota.

Nevertheless, alterations in intestinal microbiota should also be considered as a possible pathogenetic factor in MC. The role of microbiota in regulating intestinal function has increasingly been appreciated and perturbation of intestinal microbiota seems plausible in microscopic colitis. ${ }^{59}$ The anatomic sites of highest bacterial concentration in the gut (cecal and right colon) are the sites most frequently affected by inflammation. ${ }^{19}$ There is also some, albeit not robust, evidence that CC patients may benefit from treatment with probiotics. 60

\section{CO-EXPOSURE TO NSAIDS AND PPIS}

PPIs and NSAIDs are often used simultaneously, with the former frequently co-prescribed to reduce gastrointestinal injury due to the latter. Recent video capsule studies suggest ${ }^{6} 1$, 62 a very high incidence (55-70\%) of intestinal damage in healthy humans taking both NSAIDs and PPIs for 2 weeks. A more recent study performed in rats demonstrates that PPIs induced a marked exacerbation of small intestinal ulceration induced by NSAIDs, which was transferable to germ-free mice via microbiota isolated from the PPI-treated rats, suggesting an important role for microbial alterations. When PPIs were administered alone, significant changes in intestinal microbiota were observed, with $80 \%$ reduction in the levels of the beneficial Bifidobacteria spp, whereas little morphological effect was detected on the intestinal mucosa. ${ }^{63}$

Overall, it is tempting to assume that PPIs can potentially induce alterations in intestinal microbiota, albeit not to a clinically significant degree, which can in turn impair the capacity of the intestine to respond to potentially noxious agents, such as NSAIDs, known also to affect intestinal barrier function. Such a 'two-hit' theory could provide an explanation for the relevance of co-exposure to PPIs and NSAIDs in the development of

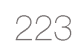


microscopic colitis. Recent results suggest a higher intake of PPIs with NSAIDs in patients with $\mathrm{MC}$ compared to controls from the general population. ${ }^{14}$ However, in the published case reports, only 2 of 20 patients were using PPI's and NSAIDs simultaneously. Further studies are warranted to provide confirmative evidence on this potential additive effect of co-ingestion.

\section{OTHER DRUGS}

A number of other drugs have been proposed to be associated with MC. Ticlopidine, for instance, has also been reported in a number of cases to be the cause of MC. The possible underlying mechanism was suggested to be related to induction of apoptosis of epithelial cells in the colonic crypts. ${ }^{64}$ Other drugs that have been associated with the induction of $\mathrm{MC}$ include ranitidine, ${ }^{65}$ acarbose, ${ }^{66}$ the venotonic drug Cyclo 3 Fort, flutamide, ${ }^{19}$ beta blockers $^{15}$ and statins. ${ }^{67}$ The association of statins with colitis has been based on an ischemic pathophysiology. ${ }^{68}$

\subsection{CONCLUSION}

Diarrhea is a frequent adverse event induced by drugs, accounting for about $7 \%$ of all adverse effects of drug therapy and over 700 drugs have been claimed to cause diarrhea. ${ }^{69}$ Drugs or their metabolites may cause diarrhea directly through their pharmacological properties or through idiosyncratic hypersensitivity reactions. Furthermore, drugs can alter the colonic microbiota and subsequently cause diarrhea.

The concept that some drugs may cause or worsen MC was first proposed in the $1990 \mathrm{~s}^{16}$, 22 Olesen et al. estimated $10 \%$ of all MC cases to be induced by drugs. ${ }^{70}$ The low frequency of $M C$ associated with drugs of different pharmacodynamics profile, on the other hand, favors the pathophysiological mechanism to be of idiosyncratic nature. Resolving of symptoms after discontinuation of a drug and reoccurrence of diarrhea after rechallenge is a strong argument for such idiosyncratic drug reaction. In a recent literature review, Beaugerie et al. suggested the use of a scoring system to determine 
the strength of evidence that associated individual drugs or drug classes with microscopic colitis. ${ }^{37}$ NSAIDs, aspirin, PPIs, ranitidine, selective serotonin reuptake inhibitors (SSRIs), ticlopidine, acarbose, and statins had high or intermediate levels of association with the disease. Carbamazepine, flutamide, and paroxetine had less well-established associations with microscopic colitis. A more recent study indeed confirmed a role for NSAIDs, SSRIs, statins and PPIs, but also bisphosphonates and beta-blockers. ${ }^{15}$ Reports have also proposed that aspirin, sertraline, simvastatin and lanzoprazole may be more likely to be associated with MC than other medications in the corresponding classes. ${ }^{15,} 37$

In principle, the causative role of a certain drug can be proven by demonstrating that a) intake of the drug precedes the manifestation of disease, b) discontinuation of a certain drug results in symptom resolution (dechallenge), c) recurrence of the disease when medication is resumed (rechallenge) and $d$ ) other causes of disease have been excluded. However, for most drugs having been associated with MC, rechallenge has not been performed or reported and the number of cases is small, such that an association by chance cannot be ruled out. Apart from case reports, the very limited number of retrospective studies assessing medication use and presence of disease generally do not allow to establish a cause-effect relationship as such studies are largely confounded by other factors, such as comorbid conditions, for which the use of certain pharmacological therapy is indicated. Furthermore, depending on the methods used for data acquisition, recall bias can largely influence drug exposure rates. Performing such studies aimed at defining certain associations in $\mathrm{MC}$ is challenging considering the higher age of patients affected and thereby frequent medication use. Such approaches should, however, be able to shed light on a positive association between the use of certain drugs and the disease condition. This enables the formulation of working hypotheses, provided that biological plausibility to ascertain a legitimate causative role of a drug is present.

Several factors can be found to explain a positive association with drug exposure and MC, apart form a cause-and-effect relationship. First, as for NSAIDs, many drugs that are associated with MC, including PPIs, induce watery diarrhea as common adverse effect. 
Thus, by causing or worsening diarrhea, use of these drugs can result in identification of $\mathrm{MC}$, rather than being the primary etiological factor.

Second, one should consider that greater awareness of physicians and subsequent increase in exposure to colonoscopy with biopsy among patients receiving these medications may merely lead to an apparent increase in the diagnosis of MC.

Third, the growing list of distinct drugs and drug classes potentially associated with MC, and largely variable time interval between start of drug use and onset of microscopic colitis symptoms, indicate the great difficulty in establishing an association between specific agents and MC.23 Nevertheless, because of the associations between $M C$ and NSAIDs, PPIs, and other medications, in individual cases, drug use and recent history should be carefully reviewed in patients with watery diarrhea or newly diagnosed MC. Agents that cause symptoms might be identified based on the temporal relationship between the timing of drug use and onset of symptoms.

The fact that most case reports of drug-induced MC concern suspected adverse drug reactions remains an inherent problem in pharmacovigilance. Adverse reactions are rarely specific for the drug and a rechallenge, which can add suggestive evidence for a causative role, is rarely ethically justified. In an attempt to solve this problem many systems have been developed for a structured and harmonized assessment of causality, the most commonly used being the WHO-Uppsala Monitoring Center ${ }^{71}$ and the Naranjo criteria. ${ }^{72}$ None of these systems, however, have been shown to produce a precise and reliable quantitative estimation of relationship likelihood resulting in a lack of international consensus regarding causality assessment.

Nevertheless, valid arguments for causality have been provided by studies showing marked improvement in symptoms or histology after stopping the drug and in some cases rechallenge resulting in symptom relapse. Overall, evidence exists to support the role of certain drugs, in particular NSAIDs and PPIs, are able to contribute to the development of MC. In terms of disease development, it is tempting to assume that $\mathrm{MC}$ induced by a 
heterogeneous group of drugs possibly shares a common multi-step pathophysiology related to the impairment of the intestinal epithelial barrier. Drug-induced impairment of the barrier may set the stage for further, as yet undetermined, insults through a luminal antigen that precipitates the disease. By virtue of interfering with intestinal homeostasis, they may potentially initiate or exacerbate ongoing unfavorable mucosal immune activation resulting in clinically manifest MC. Even though accumulating evidence suggests a role for certain drugs in disease development, it is obviously not the sole factor in MC.

In conclusion, we systematically reviewed the literature on medication use and microscopic colitis.. In individual cases, drugs such as NSAIDs and PPIs should be considered as potential etiological factors. Patients taking these drugs may therefore warrant investigation for $\mathrm{MC}$ when developing watery diarrhea. It is crucial to ascertain the temporal relationship between exposure and symptom onset to support a causative role. Following diagnosis, attempt should be made to discontinue the suspected drug. Although a number of hypotheses have been formulated with regards to potential pathophysiological mechanisms in drug-induced MC, confirmative evidence is still largely lacking. Given the wide use of these drugs among the general population and the relative rarity of the condition, drug-induced $\mathrm{MC}$ seems to be an uncommon complication, which most probably simply reflects an unfortunate idiosyncratic reaction to the particular drug. 


\section{5.}

1. Pardi DS. Microscopic colitis: an update. Inflamm Bowel Dis 2004;10:860-70.

2. Pardi DS, Smyrk TC, Tremaine WJ, Sandborn WJ. Microscopic colitis: a review. Am J Gastroenterol 2002;97:794-802.

3. Pardi DS, Kelly CP. Microscopic colitis. Gastroenterology 2011;140:1 155-65.

4. Pardi DS, Loftus EV, Jr., Smyrk TC, Kammer PP, Tremaine WJ, Schleck CD, Harmsen WS, Zinsmeister AR, Melton LJ, 3rd, Sandborn WJ. The epidemiology of microscopic colitis: a population based study in Olmsted County, Minnesota. Gut 2007;56:5048.

5. Jarnerot G, Hertervig E, Granno C, Thorhallsson E, Eriksson S, Tysk C, Hansson I, Bjorknas H, Bohr J, Olesen M, Willen R, Kagevi I, Danielsson A. Familial occurrence of microscopic colitis: a report on five families. Scand J Gastroenterol 200 1;36:95962.

6. Abdo AA, Zetler PJ, Halparin LS. Familial microscopic colitis. Can J Gastroenterol 2001;15:341-3.

7. LaSala PR, Chodosh AB, Vecchio JA, Schned LM, Blaszyk H. Seasonal pattern of onset in lymphocytic colitis. J Clin Gastroenterol 2005;39:891-3.

8. Olesen M, Eriksson S, Bohr J, Jarnerot G, Tysk C. Microscopic colitis: a common diarrhoeal disease. An epidemiological study in Orebro, Sweden, 1993-1998. Gut 2004;53:346-50.

9. Kao KT, Pedraza BA, McClune AC, Rios DA, Mao YQ, Zuch RH, Kanter MH, Wirio S, Conteas CN. Microscopic colitis: a large retrospective analysis from a health maintenance organization experience. World J Gastroenterol 2009;15:3122-7.

10. Hwang WS, Kelly JK, Shaffer EA, Hershfield NB. Collagenous colitis: a disease of pericryptal fibroblast sheath? J Pathol 1986;149:33-40.

11. Widgren S, Jlidi R, Cox JN. Collagenous colitis: histologic, morphometric, immunohistochemical and ultrastructural studies. Report of 21 cases. Virchows Arch A Pathol Anat Histopathol 1988;413:287-96.

12. Wang KK, Perrault J, Carpenter HA, Schroeder KW, Tremaine WJ. Collagenous colitis: a clinicopathologic correlation. Mayo Clin Proc 1987;62:665-71.

13. Tangri V, Chande N. Microscopic colitis: an update. J Clin Gastroenterol 2009;43:293-6.

14. Keszthelyi D, Jansen SV, Schouten GA, de Kort S, Scholtes B, Engels LG, Masclee AA. Proton pump inhibitor use is associated with an increased risk for microscopic colitis: a case-control study. Aliment Pharmacol Ther 2010;32:1124-8.

15. Fernandez-Banares F, Esteve M, Espinos JC, Rosinach M, Forne M, Salas A, Viver JM. Drug consumption and the risk of microscopic colitis. Am J Gastroenterol 2007; 102:324-30.

16. Riddell RH, Tanaka M, Mazzoleni G. Non-steroidal anti-inflammatory drugs as a possible cause of collagenous colitis: a case-control study. Gut 1992;33:683-6.

17. Huedo-Medina TB, Sanchez-Meca J, Marin-Martinez F, Botella J. Assessing heterogeneity in meta-analysis: $Q$ statistic or 12 index? Psychol Methods 2006; 11:193-206.

18. Tanner AR, Raghunath AS. Colonic inflammation and nonsteroidal anti-inflammatory 
drug administration. An assessment of the frequency of the problem. Digestion 1988;41:116-20.

19. Baert F, Wouters K, D'Haens G, Hoang P, Naegels S, D'Heygere F, Holvoet J, Louis E, Devos M, Geboes K. Lymphocytic colitis: a distinct clinical entity? A clinicopathological confrontation of lymphocytic and collagenous colitis. Gut 1999;45:375-81.

20. Goff JS, Barnett JL, Pelke T, Appelman HD. Collagenous colitis: histopathology and clinical course. Am J Gastroenterol 1997;92:57-60.

21. Bjarnason I, Hayllar J, MacPherson AJ, Russell AS. Side effects of nonsteroidal antiinflammatory drugs on the small and large intestine in humans. Gastroenterology 1993; 104:1832-47.

22. Giardiello FM, Hansen FC, 3rd, Lazenby AJ, Hellman DB, Milligan FD, Bayless TM, Yardley JH. Collagenous colitis in setting of nonsteroidal antiinflammatory drugs and antibiotics. Dig Dis Sci 1990;35:257-60.

23. Bohr J, Tysk C, Eriksson S, Abrahamsson H, Jarnerot G. Collagenous colitis: a retrospective study of clinical presentation and treatment in 163 patients. Gut 1996;39:846-51.

24. Chande N, Driman DK, Reynolds RP. Collagenous colitis and lymphocytic colitis: patient characteristics and clinical presentation. Scand J Gastroenterol 2005;40:343-7.

25. Yagi K, Nakamura A, Sekine A, Watanabe H. Nonsteroidal anti-inflammatory drugassociated colitis with a histology of collagenous colitis. Endoscopy 2001;33:62932.

26. Al-Ghamdi MY, Malatjalian DA, Veldhuyzen van Zanten S. Causation: Recurrent collagenous colitis following repeated use of NSAIDs. Can J Gastroenterol 2002; 16:861-2.

27. Jessurun J, Yardley JH, Giardiello FM, Hamilton SR, Bayless TM. Chronic colitis with thickening of the subepithelial collagen layer (collagenous colitis): histopathologic findings in 15 patients. Hum Pathol 1987;18:839-48.

28. Williams JJ, Kaplan GG, Makhija S, Urbanski SJ, Dupre M, Panaccione R, Beck PL. Microscopic colitis-defining incidence rates and risk factors: a population-based study. Clin Gastroenterol Hepatol 2008;6:35-40.

29. Etienney I, Beaugerie L, Viboud C, Flahault A. Non-steroidal anti-inflammatory drugs as a risk factor for acute diarrhoea: a case crossover study. Gut 2003;52:260-3.

30. Gleeson MH, Davis AJ. Non-steroidal anti-inflammatory drugs, aspirin and newly diagnosed colitis: a case-control study. Aliment Pharmacol Ther 2003;17:817-25.

31. Fortun PJ, Hawkey CJ. Nonsteroidal antiinflammatory drugs and the small intestine. Curr Opin Gastroenterol 2005;21:169-75.

32. Mahmud T, Scott DL, Bjarnason I. A unifying hypothesis for the mechanism of NSAID related gastrointestinal toxicity. Ann Rheum Dis 1996;55:211-3.

33. Chande N, Driman DK. Microscopic colitis associated with lansoprazole: report of two cases and a review of the literature. Scand J Gastroenterol 2007;42:530-3.

34. Hilmer SN, Heap TR, Eckstein RP, Lauer CS, Shenfield GM. Microscopic colitis associated with exposure to lansoprazole. Med J Aust 2006;184:185-6.

35. Thomson RD, Lestina LS, Bensen SP, Toor A, Maheshwari Y, Ratcliffe NR. 
Lansoprazole-associated microscopic colitis: a case series. Am J Gastroenterol 2002;97:2908-13.

36. Wilcox GM, Mattia AR. Microscopic colitis associated with omeprazole and esomeprazole exposure. J Clin Gastroenterol 2009;43:551-3.

37. Beaugerie L, Pardi DS. Review article: drug-induced microscopic colitis - proposal for a scoring system and review of the literature. Aliment Pharmacol Ther 2005;22:27784.

38. Capurso G, Marignani M, Attilia F, Milione M, Colarossi C, Zampaletta C, Di Giulio E, Delle Fave G. Lansoprazole-induced microscopic colitis: an increasing problem? Results of a prospecive case-series and systematic review of the literature. Dig Liver Dis 2011;43:380-5.

39. Forgacs I, Loganayagam A. Overprescribing proton pump inhibitors. BMJ 2008;336:2-3.

40. Mullin JM, Gabello M, Murray LJ, Farrell CP, Bellows J, Wolov KR, Kearney KR, Rudolph D, Thornton JJ. Proton pump inhibitors: actions and reactions. Drug Discov Today 2009; 14:647-60.

41. Nakamura M, Matsui H, Serizawa H, Tsuchimoto K. Lansoprazole Novel Effector Sites Revealed by Autoradiography: Relation to Helicobacter pylori, Colon, Esophagus and Others. J Clin Biochem Nutr 2007;41:154-9.

42. Murray LJ, Gabello M, Rudolph DS, Farrell CP, Morgan M, Martin AP, Underwood JC, Valenzano MC, Mullin JM. Transmucosal gastric leak induced by proton pump inhibitors. Dig Dis Sci 2009;54:1408-17.

43. Mullin JM, Valenzano MC, Whitby M, Lurie D, Schmidt JD, Jain V, Tully O, Kearney K, Lazowick D, Mercogliano G, Thornton JJ. Esomeprazole induces upper gastrointestinal tract transmucosal permeability increase. Aliment Pharmacol Ther 2008;28:1317-25.

44. Burgel N, Bojarski C, Mankertz J, Zeitz M, Fromm M, Schulzke JD. Mechanisms of diarrhea in collagenous colitis. Gastroenterology 2002;123:433-43.

45. Tagkalidis PP, Gibson PR, Bhathal PS. Microscopic colitis demonstrates a T helper cell type 1 mucosal cytokine profile. J Clin Pathol 2007;60:382-7.

46. Broeren MA, Geerdink EA, Vader HL, van den Wall Bake AW. Hypomagnesemia induced by several proton-pump inhibitors. Ann Intern Med 2009;151:755-6.

47. Cundy T, Dissanayake A. Severe hypomagnesaemia in long-term users of protonpump inhibitors. Clin Endocrinol (Oxf) 2008;69:338-41.

48. Gouraud A, Vochelle V, Descotes J, Vial T. Proton pump inhibitor-induced neutropenia: possible cross-reactivity between omeprazole and pantoprazole. Clin Drug Investig 2010;30:559-63.

49. Dury S, Nardi J, Gozalo C, Lebargy F, Deslee G. Agranulocytosis Induced by Proton Pump Inhibitors. J Clin Gastroenterol 2012.

50. Lewis SJ, Franco S, Young G, O'Keefe SJ. Altered bowel function and duodenal bacterial overgrowth in patients treated with omeprazole. Aliment Pharmacol Ther 1996; 10:557-61.

51. Williams C, McColl KE. Review article: proton pump inhibitors and bacterial overgrowth. Aliment Pharmacol Ther 2006;23:3-10.

52. Thorens J, Froehlich F, Schwizer W, Saraga E, Bille J, Gyr K, Duroux P, Nicolet M, 
Pignatelli B, Blum AL, Gonvers JJ, Fried M. Bacterial overgrowth during treatment with omeprazole compared with cimetidine: a prospective randomised double blind study. Gut 1996;39:54-9.

53. Fried M, Siegrist H, Frei R, Froehlich F, Duroux P, Thorens J, Blum A, Bille J, Gonvers JJ, Gyr K. Duodenal bacterial overgrowth during treatment in outpatients with omeprazole. Gut 1994;35:23-6.

54. Vesper BJ, Jawdi A, Altman KW, Haines GK, 3rd, Tao L, Radosevich JA. The effect of proton pump inhibitors on the human microbiota. Curr Drug Metab 2009; 10:849.

55. Spiegel BM, Chey WD, Chang L. Bacterial overgrowth and irritable bowel syndrome: unifying hypothesis or a spurious consequence of proton pump inhibitors? Am J Gastroenterol 2008;103:2972-6.

56. Drug Safety FDA. Communication: Clostridium difficile-associated diarrhea can be associated with stomach acid drugs known as proton pump inhibitors (PPIs). February 8, $2012 . \quad$ Available at:http://www.fda.gov/Drugs/DrugSafety/ucm290510.htm. Accessed May 12, 2012.

57. Lombardo L, Foti M, Ruggia O, Chiecchio A. Increased incidence of small intestinal bacterial overgrowth during proton pump inhibitor therapy. Clin Gastroenterol Hepatol 2010;8:504-8.

58. Spiegel BM. Questioning the bacterial overgrowth hypothesis of irritable bowel syndrome: an epidemiologic and evolutionary perspective. Clin Gastroenterol Hepatol 2011;9:461-9; quiz e59.

59. Gustafsson RJ, Ohlsson B, Benoni C, Jeppsson B, Olsson C. Mucosa-associated bacteria in two middle-aged women diagnosed with collagenous colitis. World $\mathrm{J}$ Gastroenterol 2012;18:1628-34.

60. Wildt S, Munck LK, Vinter-Jensen L, Hanse BF, Nordgaard-Lassen I, Christensen S, Avnstroem S, Rasmussen SN, Rumessen JJ. Probiotic treatment of collagenous colitis: a randomized, double-blind, placebo-controlled trial with Lactobacillus acidophilus and Bifidobacterium animalis subsp. Lactis. Inflamm Bowel Dis 2006; 12:395-401.

61. Maiden L, Thjodleifsson B, Theodors A, Gonzalez J, Bjarnason I. A quantitative analysis of NSAID-induced small bowel pathology by capsule enteroscopy. Gastroenterology 2005; 128:1172-8.

62. Fujimori S, Gudis K, Takahashi Y, Seo T, Yamada Y, Ehara A, Kobayashi T, Mitsui K, Yonezawa M, Tanaka S, Tatsuguchi A, Sakamoto C. Distribution of small intestinal mucosal injuries as a result of NSAID administration. Eur J Clin Invest 2010;40:50410.

63. Wallace JL, Syer S, Denou E, de Palma G, Vong L, McKnight W, Jury J, Bolla M, Bercik P, Collins SM, Verdu E, Ongini E. Proton pump inhibitors exacerbate NSAIDinduced small intestinal injury by inducing dysbiosis. Gastroenterology 2011;141:1314-22, 1322 e 1-5.

64. Berrebi D, Sautet A, Flejou JF, Dauge MC, Peuchmaur M, Potet F. Ticlopidine induced colitis: a histopathological study including apoptosis. J Clin Pathol 1998;51:280-3.

65. Beaugerie L, Patey N, Brousse N. Ranitidine, diarrhoea, and lymphocytic colitis. Gut 1995;37:708-11. 
66. Piche T, Raimondi V, Schneider S, Hebuterne X, Rampal P. Acarbose and lymphocytic colitis. Lancet 2000;356:1246.

67. Mukhopadhya A, Gilmour H, Plevris J. Pravastatin-induced colitis. Eur J Gastroenterol Hepatol 2008;20:810-2.

68. Leung FW, Lieberman J, Fagen N, Kasimian D, Wick R. Colonoscopic features of simvastatin-induced colitis suggest ischemia as an etiologic mechanism. Gastrointest Endosc 2005;62:175-8.

69. Chassany O, Michaux A, Bergmann JF. Drug-induced diarrhoea. Drug Saf 2000;22:53-72.

70. Olesen M, Eriksson S, Bohr J, Jarnerot G, Tysk C. Lymphocytic colitis: a retrospective clinical study of 199 Swedish patients. Gut 2004;53:536-41.

71. Centre TUM. The use of the WHO-UMC system for standardised case causality assessment.

72. Naranjo CA, Busto U, Sellers EM, Sandor P, Ruiz I, Roberts EA, Janecek E, Domecq C, Greenblatt DJ. A method for estimating the probability of adverse drug reactions. Clin Pharmacol Ther 1981;30:239-45. 
Proton pump inhibitor use is associated with an increased risk for microscopic colitis: a case-control study

D. Keszthelyi, SV. Jansen, GA. Schouten, S. de Kort, B. Scholtes, LG. Engels, AAM. Masclee

Alimentary Pharmacology and Therapeutics 2010 Nov;32(9):1124-8

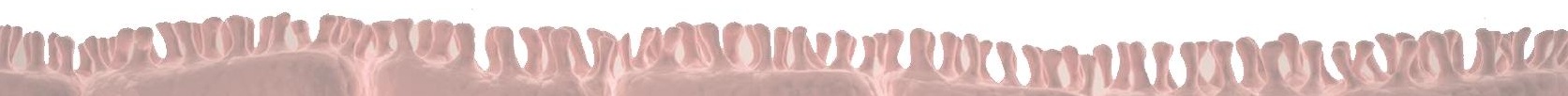




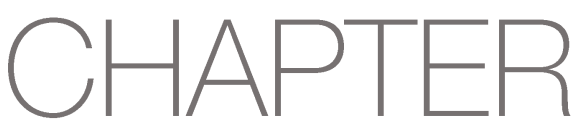

Drug exposure in microscopic colitis 


\section{ABSTRACT}

Background: Microscopic colitis (MC) causes chronic watery diarrhea. Recent studies have suggested an etiological role for various medications, including proton pump inhibitors, in the pathogenesis of MC.

Aim: To determine whether an association exists between microscopic colitis and proton pump inhibitor use in patients with documented microscopic colitis versus age and sexmatched controls.

Methods: In this retrospective case-control study. Cases of microscopic colitis from a secondary and tertiary referral medical center diagnosed in the last 5 years were reviewed. Demographic characteristics, clinical, histological and endoscopic records, as well as exposure to PPIs and NSAIDs were assessed. Controls from the population were matched to cases by gender and by age.

Results: During the investigated period, 136 cases were identified in both hospitals. Of these, 95 cases of MC were retrieved for detailed analysis. Exposure to proton pump inhibitors at the time of the histological diagnosis was significantly higher in MC patients than in controls (38\% vs 13\%, p<0.001; adjusted OR of 4.5 [95\% Cl 2.0-9.5]).

Conclusion: This observation confirms the presumed association between microscopic colitis and PPI use and supports the possible etiological role of PPI exposure in the development of microscopic colitis. 


\subsection{INTRODUCTION}

The term 'microscopic colitis' (MC) was introduced in 1980 to describe patients with chronic watery diarrhea characterized by normal radiological and endoscopic mucosal appearance but microscopic abnormalities in the colonic mucosa. ' Currently, 'microscopic colitis' is used as an umbrella term for two separate entities: collagenous colitis and lymphocytic colitis. The number of intraepithelial CD8+ T-lymphocytes is increased in both collagenous and lymphocytic colitis, exceeding $20 \%$ of the surface epithelial cells. This is accompanied by superficial epithelial damage and a variable inflammatory infiltrate in the lamina propria. In addition, collagenous colitis is also characterized by a thickened subepithelial collagen band, which exceeds $10 \mu \mathrm{m}$. These histological characteristics are not pathognomic as they may occur in various inflammatory conditions in the colon. ${ }^{2}$

In the past, $\mathrm{MC}$ was thought to be a rare disorder. However, it has recently become apparent that MC accounts for up to $13 \%$ of patients investigated for chronic diarrhea, particularly in middle-aged and elderly patients. ${ }^{2}, 3$ Pardi et al. reported an increasing annual incidence rate of 7.1 for collagenous colitis and of 12.6 for lymphocytic colitis per 1000000 in the period of 1998 to 2001 . $^{3}$ The cause of MC remains unknown. One postulated hypothesis points to increased colonic permeability in MC patients, thereby allowing luminal antigens to enter the lamina propria and elicit an immune and inflammatory reaction. Environmental risk factors, such as drugs, have been suggested as causes or triggers of this process. ${ }^{4}$ These drugs include non-steroidal antiinflammatory drugs (NSAIDs), selective serotonin reuptake inhibitors (SSRIs), biphosphonates, beta-blockers, statins and proton pump inhibitors (PPIs). ${ }^{5}$

Recently, several case reports have been published which further highlight the association between PPI use and development of MC.6-9 Initially, lansoprazole has been found to be associated with a high likelihood of inducing $M C .^{10} \mathrm{~A}$ recent study also points to similar effects in case of exposure to omeprazole and esomeprazole. ${ }^{9}$ PPIs are one of the most frequently prescribed classes of medications worldwide because they combine a high level of efficacy with low toxicity. In 2006, expenditure on these drugs was 10 billion USD 


\section{Chapter 10}

globally. ${ }^{11}$ In the five years since the introduction of esomeprazole in 2001, prescriptions for PPIs have doubled. ${ }^{11}$ A recent socio-demographic study from The Netherlands found that $11.8 \%$ of the population had at least one prescription for a PPI in the year $2006 .^{12}$ It is noteworthy, that the increasing incidence of $\mathrm{MC}^{3}$ seems to parallel the rising use of PPIs. In this report, we analyze the possible association between MC and PPI use in patients with documented $\mathrm{MC}$ versus age and sex-matched controls.

\subsection{METHODS}

\section{PATIENTS}

We performed a retrospective, two-center, observational, case-control study. Patients with $\mathrm{MC}$ were identified by reviewing the pathology database and medical records at the Maastricht University Medical Center, The Netherlands, a tertiary medical center, and at Orbis Medical Center, Sittard, The Netherlands, a secondary medical center, from November 2005 to November 2009. Diagnosis was based on clinical symptoms, data from histology and endoscopy. Following identification, written informed consent was obtained from the patients to assess medical and pharmacy records. Information on prescribed medication for the period of two years prior to the histological diagnosis was obtained from the pharmacy database. All patients having had at least one prescription for a PPI (esomeprazole, omeprazole, lansoprazole, pantoprazole or rabeprazole) in 180 days prior to the time of histological diagnosis were considered exposed to PPIs. Similarly, we investigated exposure to NSAIDs, a known risk factor for MC, as well as exposure to benzodiazepines (BDZs), diuretics and ACE inhibitors, which have not been associated with $\mathrm{MC}$, as control medication. Use of low-dose aspirin (up to $300 \mathrm{mg} /$ day) was not considered as NSAID exposure. The reason for selecting a time window of 180 days between drug exposure and diagnosis of microscopic colitis was the considerable delay that can occur between initial symptom onset and eventual endoscopic examination with mucosal biopsy sampling. Also, comorbidity was also assessed by reviewing medical records. 


\section{CONTROLS}

The control group consisted of 355 individuals randomly selected from the general population in Maastricht, The Netherlands, derived from the municipal residential database. Controls received questionnaires regarding their current medical status and drug exposure. Ninety-five controls were matched randomly to cases by gender and by age (within 1 year). Also, comorbidity was assessed by reviewing medical records.

\section{STATISTICAL ANALYSIS}

Statistical analysis was done by performing $X^{2}$ test and generalized linear model for binomial regression to calculate ORs and $95 \%$ Cls using SPSS, version 17.0 (Chicago, Illinois). Statistical significance was predetermined as $p<0.05$.

\subsection{RESULTS}

During the investigated period, 136 cases were identified in both hospitals. Of these, 95 cases of MC were retrieved for detailed analysis. No detailed pharmacy records were available for the remaining 41 cases. Mean age of MC patients was $58 \pm 1$ yrs, $66 \%$ were women. We observed a 1:1.07 ratio for lymphocytic vs collagenous colitis. Cases did not differ significantly in BMl from controls ( $25 \pm 5 \mathrm{vs}$. $26 \pm 4 \mathrm{~kg} / \mathrm{m}^{2}$, mean $\pm \mathrm{SD}$ ). Exposure to both PPIs and NSAIDs at the time of the histological diagnosis was significantly higher in patients than in controls (PPI 38\% vs $13 \%, p<0.001$ and NSAIDs $20 \%$ vs $5 \%, p=0.002$ vs control, respectively). Co-exposure to both PPIs and NSAIDs was also significantly higher in $\mathrm{MC}$ patients compared to controls ( $8 \%$ vs $0 \%, p=0.007$ ). After adjustment for concomitant exposure to NSAIDs, BDZs, diuretics and ACE inhibitors as covariates, an adjusted OR of 4.5 [95\% $\mathrm{Cl}$ 2.0-9.5] was found for PPI exposure (see Table 1). There were no differences observed in exposure to PPIs or NSAIDs with regard to the histological subtype (collagenous or lymphocytic). No significant association was found between gender and histological subtype or drug exposure. 


\begin{tabular}{|c|c|c|c|}
\hline Drug & MC patients & Controls & OR $[95 \% \mathrm{Cl}]$ \\
\hline $\mathrm{PPI}$ & $37.9 \%$ & $12.6 \%$ & $4.5[2.0-9.5]$ \\
\hline NSAID & $20.4 \%$ & $5.3 \%$ & $2.3[0.8-6.5]$ \\
\hline $\mathrm{BDZ}$ & $12.8 \%$ & $8.4 \%$ & $1.2[0.4-3.7]$ \\
\hline Diuretics & $12.8 \%$ & $8.4 \%$ & $1.4[0.5-4.4]$ \\
\hline ACE-I & $7.7 \%$ & $5.3 \%$ & $1.8[0.5-4.4]$ \\
\hline PPI+NSAID & $8.0 \%$ & $0 \%$ & N.A. \\
\hline
\end{tabular}

Table 1: Exposure to PPIs and NSAIDs in microscopic colitis (MC) patients and controls.

Considering the fact that the pharmacy records were not available or obtainable in case of 41 patients, calculations have been done according to the 'worst-case scenario.' This would mean that of the 41 patients, from which no data on medication was obtainable or available, the assumption was made that none were taking PPIs prior to the histological diagnosis ( 0 out of 41 , overall 36 out of 136). As for the control population for these additional 41 patients, we presumed that the consumption of PPIs would be equal to what we observed in the control population (13\%, approximately 5 out of 41 , overall 17 out of 136). Repeating the binary logistic regression analysis with these fictive numbers results in an OR of 2.5 [Cl 1.3-4.7] for PPI exposure.

Regarding the type of PPI patients were exposed to, we found the following distribution: $40 \%$ omeprazole, $23 \%$ esomeprazole, $28 \%$ pantoprazole, $6 \%$ rabeprazole, $3 \%$ patients used lansoprazole (see Table 3).

\begin{tabular}{r|ccc} 
& MC patients & Controls & $p$ value $\left(x^{2}\right)$ \\
\hline Celiac disease & $3.2 \%$ & $0 \%$ & 0.25 \\
Rheumatoid arthritis & $6.3 \%$ & $0 \%$ & 0.03 \\
Hypothyroidism & $5.2 \%$ & $0 \%$ & 0.06 \\
Irritable bowel syndrome & $8.4 \%$ & $6.3 \%$ & 0.78
\end{tabular}

Table 2: Comorbidities among microscopic colitis (MC) patients and controls. 
Celiac disease and autoimmune diseases have been reported to be associated with MC. ${ }^{4}$ In our patient population, celiac disease was diagnosed in $3 \%$ of patients with MC. This rate is comparable to previous reports. ${ }^{3}$ Rheumatoid arthritis was found in $6 \%$, hypothyroidism in $5 \%$ of MC patients (see Table 2). Multivariate logistic regression analyses with comorbidities as covariates resulted in an OR of 5.8 [2.5-13.5] for PPI exposure. This implies that patients with comorbidity have an even higher risk for developing MC upon exposure to PPIs.

\begin{tabular}{r|cc} 
Type of PPI & MC patients & Controls \\
\hline Omeprazole & $40.0 \%$ & $50.0 \%$ \\
Esomeprazole & $22.8 \%$ & $30.0 \%$ \\
Pantoprazole & $28.6 \%$ & $20.0 \%$ \\
Rabeprazole & $5.7 \%$ & $0 \%$ \\
Lansoprazole & $2.8 \%$ & $0 \%$
\end{tabular}

Table 3: Types of PPls used among microscopic (MC) patients and controls.

\subsection{DISCUSSION}

Recent studies have emphasized the association between exposure to PPIs and occurrence of MC. Our findings confirm the importance of considering PPI use as a possible etiological factor for MC. However, case-control studies generally do not allow interpretation with respect to a causal relation. It should be noticed that our study has a number of limitations. First, the population in this study is relatively small and pharmacy records were not available or obtainable in about $30 \%$ of the cases in the period reviewed. Nevertheless, calculations according to a 'worst-case scenario' in which none of the remaining $41 \mathrm{MC}$ patients were exposed to PPIs in the studied period still show a significant risk for developing MC. Second, use of over-the-counter medication could not be assessed. Third, the time window between symptom onset and histological diagnosis may vary largely between $\mathrm{MC}$ patients, which makes assessment of drug exposure more difficult. For that reason, we assessed drug intake for a longer time window preceding the diagnosis. Fourth, for similar reasons, history of gastrointestinal symptoms could 
neither be assessed in this time period. Further studies, when possible on larger samples of patients, are needed to confirm our observations.

The pathophysiological mechanisms underlying PPI-related induction of MC are poorly understood. Proton pumps ( $\mathrm{H}^{+} / \mathrm{K}^{+}$ATPases) are present not only on gastric epithelium but also on colonic epithelium where they contribute to whole body potassium homeostasis. ${ }^{13}$ Inhibition of the colonic proton pumps may therefore affect local electrolyte balance and compromise fluid acidification, which can possibly affect immune reactions in the colonic mucosa.

Proteins other than $\mathrm{H}^{+} / \mathrm{K}^{+}$ATPase have also been reported as targets for PPIs. Omeprazole and lansoprazole have been observed to induce smooth muscle relaxation and to inhibit contractile activity. ${ }^{13}$ This effect on contractile systems could also affect tight junction functionality since tight junction proteins are directly linked to the actinomyosin cytoskeleton. Therefore, conformational changes in the cytoskeleton of epithelial cells may result in alterations in the function of the tight junction, which leads to increased paracellular permeability. As a result, luminal contents can more easily penetrate the lamina propria causing an immune and/or inflammatory reaction. Esomeprazole has been shown to increase paracellular permeability in the upper gastrointestinal tract in vitro $^{14}$ and in vivo in humans. ${ }^{15}$ Increased paracellular permeability has been observed in $\mathrm{MC}$ and is considered a key component in the induction of diarrhea. ${ }^{16}$ Accordingly, expression of the tight junction proteins occludin, claudin-4 and zonuline-1 (ZO-1) was found to be decreased in mucosal biopsy specimens from MC patients. 16, 17 We speculate that a direct or indirect effect of PPIs on colonic tight junctions and paracellular permeability may explain the association between PPI use and $\mathrm{MC}$ observed in this study.

Although not directly linked to the pathogenesis of $\mathrm{MC}$, recent case reports have related long-time PPI exposure to severe hypomagnesemia. ${ }^{18,19}$ This effect has been described for omeprazole, esomeprazole, pantoprazole and lansoprazole as well. ${ }^{18}$ It has been proposed that PPIs induce a defect in the active absorption of magnesium in the intestine. 
This may result from an effect of PPIs on the tight junction proteins or on the TRPM6 and 7 channels (transient receptor potential melastin 6 and 7). The latter are key molecules involved in active magnesium absorption. Either changes in intestinal $\mathrm{pH}$ induced by PPIs may affect channel functions, or subjects who are heterozygous carriers of TRPM6/7 mutations are more susceptible to developing adverse reactions to PPIs. ${ }^{19}$ Presently, we cannot exclude that effects of PPIs on magnesium absorption also play a role in the pathogenesis of $M C$.

In addition, it has long been established that PPI therapy can alter intestinal microbial profiles. ${ }^{20}$ PPIs can directly affect intestinal microbiota through inhibition of the $\mathrm{H}^{+} / \mathrm{K}^{+}$ ATPase which can inhibit microbial growth. Several bacteria, including $H$. pylori and $S$. pneumoniae, as well as fungi such as $C$. albicans, contain $\mathrm{H}^{+} / \mathrm{K}^{+}$ATPase in their plasma membranes which are highly homologous to their human counterparts. ${ }^{21}$ On the other hand, increase of intestinal pH can result in a diminished host defence against certain bacteria. Profound acid suppression increases the risk of enteric infections in susceptible individuals caused by Shigella, Salmonella, Yersinia or Clostridium difficile.22 The use of PPIs may also promote the expansion and colonization of $C$. difficile by its recognized potential to induce small bowel bacterial overgrowth with anaerobic colonic organisms. ${ }^{20}$ Many believe that PPI use may therefore directly contribute to $C$. difficile and other bacterial infections. Certainly, further research is needed to clarify the exact effects of PPIs on human intestinal microbiota. Nevertheless, alterations in intestinal microbiota should also be considered as a possible pathogenetic aspect in MC.

In conclusion, the observations of our case-control study confirm the presumed association between MC and PPI use and support the possible etiological role of PPI exposure in the development of MC. When patients are prescribed PPIs to treat upper gastrointestinal disorders, it is important to recognize that PPIs are interacting at multiple targets. As a result, MC may occur as an adverse reaction of PPIs and PPIs should be considered as a possible cause of diarrhea in this population. 


\subsection{REFERENCES}

1. Read NW, Krejs GJ, Read MG, Santa Ana CA, Morawski SG, Fordtran JS. Chronic diarrhea of unknown origin. Gastroenterology 1980;78:264-71.

2. Pardi DS, Smyrk TC, Tremaine WJ, Sandborn WJ. Microscopic colitis: a review. Am J Gastroenterol 2002;97:794-802.

3. Pardi DS, Loftus EV, Jr., Smyrk TC, Kammer PP, Tremaine WJ, Schleck CD, Harmsen WS, Zinsmeister AR, Melton LJ, 3rd, Sandborn WJ. The epidemiology of microscopic colitis: a population based study in Olmsted County, Minnesota. Gut 2007;56:5048.

4. Tangri V, Chande N. Microscopic colitis: an update. J Clin Gastroenterol 2009;43:293-6.

5. Fernandez-Banares F, Esteve M, Espinos JC, Rosinach M, Forne M, Salas A, Viver JM. Drug consumption and the risk of microscopic colitis. Am J Gastroenterol 2007; 102:324-30.

6. Chande N, Driman DK. Microscopic colitis associated with lansoprazole: report of two cases and a review of the literature. Scand J Gastroenterol 2007;42:530-3.

7. Hilmer SN, Heap TR, Eckstein RP, Lauer CS, Shenfield GM. Microscopic colitis associated with exposure to lansoprazole. Med J Aust 2006;184:185-6.

8. Thomson RD, Lestina LS, Bensen SP, Toor A, Maheshwari Y, Ratcliffe NR. Lansoprazole-associated microscopic colitis: a case series. Am J Gastroenterol 2002;97:2908-13.

9. Wilcox GM, Mattia AR. Microscopic colitis associated with omeprazole and esomeprazole exposure. J Clin Gastroenterol 2009;43:551-3.

10. Beaugerie L, Pardi DS. Review article: drug-induced microscopic colitis - proposal for a scoring system and review of the literature. Aliment Pharmacol Ther 2005;22:27784.

11. Forgacs I, Loganayagam A. Overprescribing proton pump inhibitors. BMJ 2008;336:2-3.

12. van Boxel OS, Hagenaars MP, Smout AJ, Siersema PD. Socio-demographic factors influence chronic proton pump inhibitor use by a large population in the Netherlands. Aliment Pharmacol Ther 2009;29:571-9.

13. Mullin JM, Gabello M, Murray LJ, Farrell CP, Bellows J, Wolov KR, Kearney KR, Rudolph D, Thornton JJ. Proton pump inhibitors: actions and reactions. Drug Discov Today 2009; 14:647-60.

14. Murray LJ, Gabello M, Rudolph DS, Farrell CP, Morgan M, Martin AP, Underwood JC, Valenzano MC, Mullin JM. Transmucosal gastric leak induced by proton pump inhibitors. Dig Dis Sci 2009;54:1408-17.

15. Mullin JM, Valenzano MC, Whitby M, Lurie D, Schmidt JD, Jain V, Tully O, Kearney K, Lazowick D, Mercogliano G, Thornton JJ. Esomeprazole induces upper gastrointestinal tract transmucosal permeability increase. Aliment Pharmacol Ther 2008;28:1317-25.

16. Burgel N, Bojarski C, Mankertz J, Zeitz M, Fromm M, Schulzke JD. Mechanisms of diarrhea in collagenous colitis. Gastroenterology 2002;123:433-43. 
17. Tagkalidis PP, Gibson PR, Bhathal PS. Microscopic colitis demonstrates a T helper cell type 1 mucosal cytokine profile. J Clin Pathol 2007;60:382-7.

18. Broeren MA, Geerdink EA, Vader HL, van den Wall Bake AW. Hypomagnesemia induced by several proton-pump inhibitors. Ann Intern Med 2009;151:755-6.

19. Cundy T, Dissanayake A. Severe hypomagnesaemia in long-term users of protonpump inhibitors. Clin Endocrinol (Oxf) 2008;69:338-41.

20. Lewis SJ, Franco S, Young G, O'Keefe SJ. Altered bowel function and duodenal bacterial overgrowth in patients treated with omeprazole. Aliment Pharmacol Ther 1996; 10:557-61.

21. Vesper BJ, Jawdi A, Altman KW, Haines GK, 3rd, Tao L, Radosevich JA. The effect of proton pump inhibitors on the human microbiota. Curr Drug Metab 2009;10:849.

22. Spiegel BM, Chey WD, Chang L. Bacterial overgrowth and irritable bowel syndrome: unifying hypothesis or a spurious consequence of proton pump inhibitors? Am J Gastroenterol 2008; 103:2972-6. 
Increased proton pump inhibitor and NSAID exposure in irritable bowel syndrome: results from a case-control study

D. Keszthelyi, GH. Dackus, GM. Masclee, JW. Kruimel, AAM. Masclee BMC Gastroenterology 2012 Sep 5;12:121

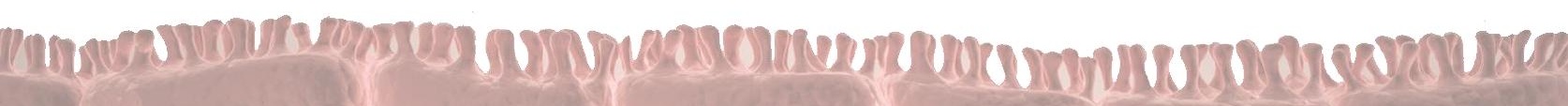


Part IV. Intestinal barrier function: effect of drugs in disease development

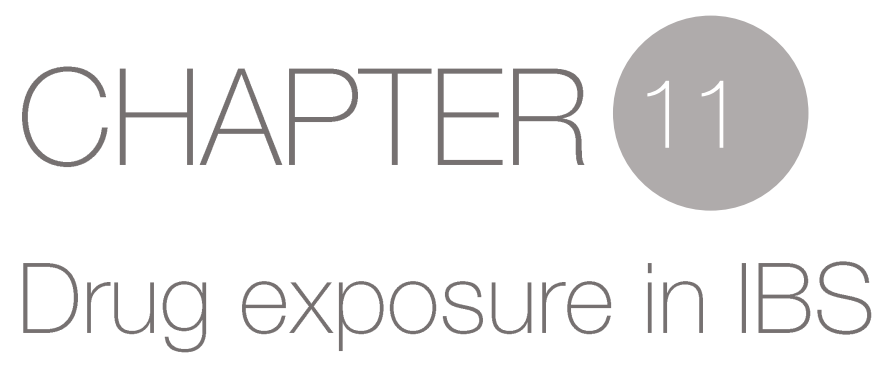

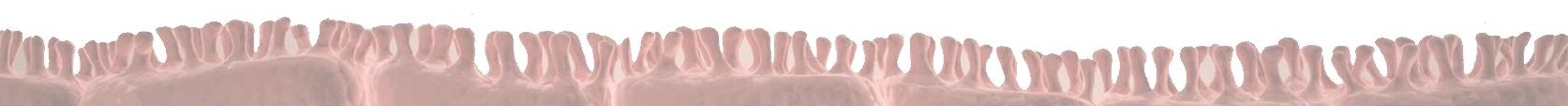




\section{ABSTRACT}

Background: Patients with irritable bowel syndrome (IBS) seen by a gastroenterologist often utilize medications that may alter intestinal homeostasis. The question arises whether exposure to these drugs is associated with the development of IBS symptoms. Aim of this study was therefore to assess the use of PPIs and NSAIDs in patients with IBS versus controls.

Methods: Cases of IBS from the last 5 years were reviewed. All patients having had at least one prescription for a particular drug (PPIs, NSAIDs, SSRIs, diuretics, ACE inhibitors) in the 6 months prior to the time of initial symptom onset were considered exposed. The control group consisted of individuals randomly selected from the general population.

Results: 287 cases of IBS were retrieved for analysis together with 287 age and sexmatched controls. Exposure to PPIs and NSAIDs was significantly higher in IBS patients, whereas no association between ACE inhibitor use and IBS was found. PPIs were not significantly associated when excluding patients with gastrointestinal reflux disease or functional dyspepsia. Exposure to SSRIs was also positively associated with IBS, but only when patients with psychiatric comorbidity were included in the analyses.

Conclusions: Medications that may alter intestinal homeostasis such as NSAIDs and PPIs were more frequently used in IBS patients compared to controls. This association might be relevant for everyday clinical practice, but it is remains to be elucidated whether this association is of etiological nature. 


\subsection{BACKGROUND}

Irritable bowel syndrome (IBS) is a condition characterized by recurrent abdominal pain or discomfort accompanied by abnormalities in bowel habits. ${ }^{1}$ It leads to considerable morbidity as it is associated with tripling of missed work-days and doubling of illnessrelated costs. ${ }^{1}$ National Health Service costs in the UK are 60\% higher in IBS patients than in non-IBS control patients. ${ }^{2}$ As care-seeking behavior can be characteristic for IBS patients, these patients are also prone to suffer from polyphragmasia and polypharmacia.

Since the pathogenesis of IBS is still poorly understood, the question arises whether the intake of medication can contribute to or trigger onset of symptoms in IBS patients, as these patients often utilize medications that may potentially alter intestinal homeostasis. One postulated hypothesis points to increased intestinal permeability in IBS patients induced by exposure to certain medication, such as non-steroidal anti-inflammatory drugs (NSAIDs), thereby allowing luminal antigens to enter the lamina propria and eliciting an immune and inflammatory reaction. ${ }^{3}$ In particular, proton pump inhibitors (PPIs) have also been suggested to be involved in the pathogenesis of IBS, primarily through alteration of intestinal microbiota composition. ${ }^{4}$ PPIs are one of the most frequently prescribed classes of medication worldwide because they combine a high level of efficacy with low toxicity. In 2009, expenses for omeprazole and esomeprazole only reached $€ 150$ million in the Netherlands. ${ }^{5}$ In the five years since the introduction of esomeprazole in 2001 , prescriptions for PPIs have doubled. ${ }^{6}$ With 5 million prescriptions, omeprazole became the second most frequently prescribed medication in the Netherlands in 2009.5 A recent socio-demographic study from the Netherlands found that $11.8 \%$ of the general population had at least one prescription for a PPI in the year 2006.7 It has also been demonstrated that in IBS patients, the use of PPIs generates an excess health cost of $€ 80$ per case per year. 8

We have recently demonstrated that exposure to PPIs was associated with increased risk for developing microscopic colitis, a condition known to share symptoms and clinical features with IBS ${ }^{10}$ and to be associated with changes in intestinal permeability. ${ }^{11}$ PPIs 


\section{Chapter 11}

have not only been demonstrated to be able to alter intestinal barrier function, ${ }^{12}$ but also to induce profound changes in intestinal microbiota composition, ${ }^{13}$ which in turn may also lead to secondary changes in epithelial integrity. ${ }^{14}$ It has therefore also been hypothesized that PPIs use is associated with IBS through causing small intestinal bacterial overgrowth (SIBO).. ${ }^{4}$ Due to these presumed alterations in intestinal physiology induced by PPI and/or NSAID use, we postulate that PPIs and NSAIDs may play an etiological role in the development of IBS. More specifically, we hypothesize that patients with IBS will be more often exposed to drugs affecting intestinal homeostasis (PPIs and NSAIDs) at time of presentation with symptoms. In this study, we aimed to establish a relationship between PPI or NSAID use and IBS by comparing exposure these drugs in comparison to control group from the general population.

\subsection{METHODS}

The study was approved by the Medical Ethics Committee of the Maastricht University Medical Centre+ (MUMC), Maastricht, the Netherlands (reference number 0904015), and performed in full accordance with the European directive 91/507/EEG, and the Declaration of Helsinki (as amended in Tokyo, Venice, Hong Kong, Somerset West and Edinburgh. Note of clarification added in Washington and Tokyo).

\section{PATIENTS}

We performed a retrospective, observational, case-control study at the Division of Gastroenterology-Hepatology at the Maastricht University Medical Center, the Netherlands, a tertiary and regionally also secondary referral medical center. Patients presenting with symptoms characteristic for IBS who had been referred to a gastroenterologist at our outpatient clinic by general practitioners or other medical specialists for diagnostic work up and therapy were identified by reviewing medical records in the period from May 2006 to November 2010. Symptoms included abdominal pain and discomfort associated with diarrhea or constipation, bloating and abdominal distension. At the time of the index visit to our outpatient clinic, the duration of the 
symptoms was assessed. In case of patients having had symptoms for at least 6 months, the diagnosis of IBS was made based on Rome III criteria at the time of the index visit. In case of patients having IBS symptoms for less than 6 months, diagnosis according to Rome III criteria was confirmed using questionnaires sent to patients to assess symptom duration following initial symptom onset. Only patients fulfilling Rome III criteria were included in the investigation.

Written informed consent was obtained from patients to obtain detailed information of their pharmacy records. Their medical records were reviewed to assess medical history and comorbidities. Information on prescribed medication for the period of two years prior to the index visit was obtained from the pharmacy database. All patients having had at least one prescription for a period of four weeks for a PPI (esomeprazole, omeprazole, pantoprazole, lansoprazole or rabeprazole) in a dose of $20 \mathrm{mg}$ (for lansoprazole $15 \mathrm{mg}$ ) or more in the 180 days prior to the time of the initial onset of symptoms were considered exposed to PPIs. Patients exposed to PPIs only in the period after initial onset of symptoms were not included in the analyses. Similarly, we investigated exposure to NSAIDs (as drugs known to alter intestinal barrier function). The following doses were considered as minimal drug exposure: diclofenac $12.5 \mathrm{mg}$, ibuprofen $200 \mathrm{mg}$, ketoprofen $100 \mathrm{mg}$, indomethacin $25 \mathrm{mg}$, aceclofenac $100 \mathrm{mg}$, nabumetone $500 \mathrm{mg}$, naproxen 250 $\mathrm{mg}$, aspirin $500 \mathrm{mg}$. COX-2 inhibitors were not included in the analyses. Also, exposure to selective serotonin reuptake inhibitors (SSRIs, drugs frequently prescribed to IBS patients) and as control medication exposure to diuretics and angiotensin converting enzyme (ACE) inhibitors, drugs that have not been associated with IBS, was investigated. The rationale for selecting a time window of 180 days between drug exposure and diagnosis of IBS was the minimum of six months duration of symptoms needed for IBS diagnosis (Rome III criteria) and the considerable delay that may occur between initial symptom onset and referral with eventual diagnosis by a gastroenterologist. Psychiatric comorbidity was defined as evidence of depression or anxiety disorder in medical history as diagnosed according to the DSM IV. 


\section{CONTROLS}

The control group consisted of 408 individuals randomly selected from the general population in Maastricht, the Netherlands. Residents with a permanent address in Maastricht were eligible for selection. Potential controls were selected by a random computerized selection from the municipality residential register. Controls received questionnaires regarding their current medical status and drug exposure. Individuals who self-admitted to being diagnosed with IBS were excluded. Controls for analyses $(n=287)$ were selected from this group and matched to IBS cases by age (within 1 year) and gender.

\section{STATISTICAL ANALYSIS}

Statistical analyses were performed using $X^{2}$ test to compare gender and comorbidities. Independent Student's t test was used to compare age and BMI. Generalized linear model for binomial regression also adjusted for comorbidities (psychiatric, gastrointestinal reflux disease [GERD], functional dyspepsia [FD], rheumatoid disorders fibromyalgia) was used to calculate ORs and $95 \%$ Cls using SPSS, version 20.0 (Chicago, IL). Statistical significance was predetermined as $p<0.05$.

\subsection{RESULtS}

During the investigated period, 521 cases were identified as having IBS according the Rome III criteria. From these, a total of 287 cases gave informed consent to assess their pharmacy records. These cases of IBS along with 287 randomly selected age and sexmatched controls were identified and retrieved for detailed analysis. For demographic characteristics, see Table 1. Distribution of IBS subtypes was as follows: $38 \%$ diarrheapredominant (IBS-D), 30\% constipation-predominant (IBS-C) and 32\% mixed subtype (IBSM). A significantly higher numer of IBS had comorbid conditions (FD or GERD, pyschatric condition, fibromyalgia, rheumatoid arthritis), compared to controls (see Table 2, all p $<0.0011)$. 
Exposure to PPIs and NSAIDs was significantly higher in IBS patients (see Table 2). Coexposure to PPIs and NSAIDs was also significantly higher in IBS $\left(7.6 \%\right.$ vs $0 \%, X^{2}=17.4$, p<0.001). Of the patients using NSAIDs (59/287), $37 \%$ also used PPIs at the same time (22/287). See Table 3 for a summary of the type of PPIs and NSAIDs patients were exposed to.

\begin{tabular}{r|lll} 
& IBS patients & Controls & P value \\
& $\mathrm{N}=287$ & $\mathrm{~N}=287$ & \\
\hline Age (years) & $36.7 \pm 16$ & $37.4 \pm 16$ & 0.64 \\
Gender & $75 \%$ female & $75 \%$ female & 1.00 \\
BMI $\left(\mathrm{kg} / \mathrm{m}^{2}\right)$ & $25.8 \pm 3.4$ & $24.7 \pm 5.1$ & 0.06
\end{tabular}

Table 1: Demographic characteristics of irritable bowel syndrome (IBS) patients and controls.

IBS patients more frequently used SSRIs (Table 2). However, no association with SSRI use was found when correcting for psychiatric comorbidity. When patients with psychiatric co-morbidity were excluded from analysis, ORs for PPIs, NSAIDs diuretics and ACE inhibitors remained unchanged, whereas SSRI exposure proved not to be associated with IBS (Table 2).

No association between ACE inhibitor or diuretic use and IBS was found (Table 2). No significant association was found with regards to IBS-subtype (diarrhea, constipation or mixed) and drug exposure.

As PPIs are most frequently prescribed for upper $\mathrm{GI}$ tract complaints originating primarily from FD or GERD, analyses were repeated with exclusion of patients and controls with documented comorbid GERD or dyspepsia. These analyses showed similar ORs for NSAIDs, SSRIs, diuretics and ACE inhibitors. However, PPI exposure proved not be associated with the disorder in the group of IBS patients without comorbid GERD or dyspepsia (OR 2.1 [0.8-5.4]). Similarly, as NSAIDs are often prescribed for rheumatic disorders, analyses were also conducted with the exclusion of patients and controls with 


\section{Chapter 11}

documented rheumatoid arthritis. These analyses showed similar ORs to the analysis with the inclusion of all subjects.

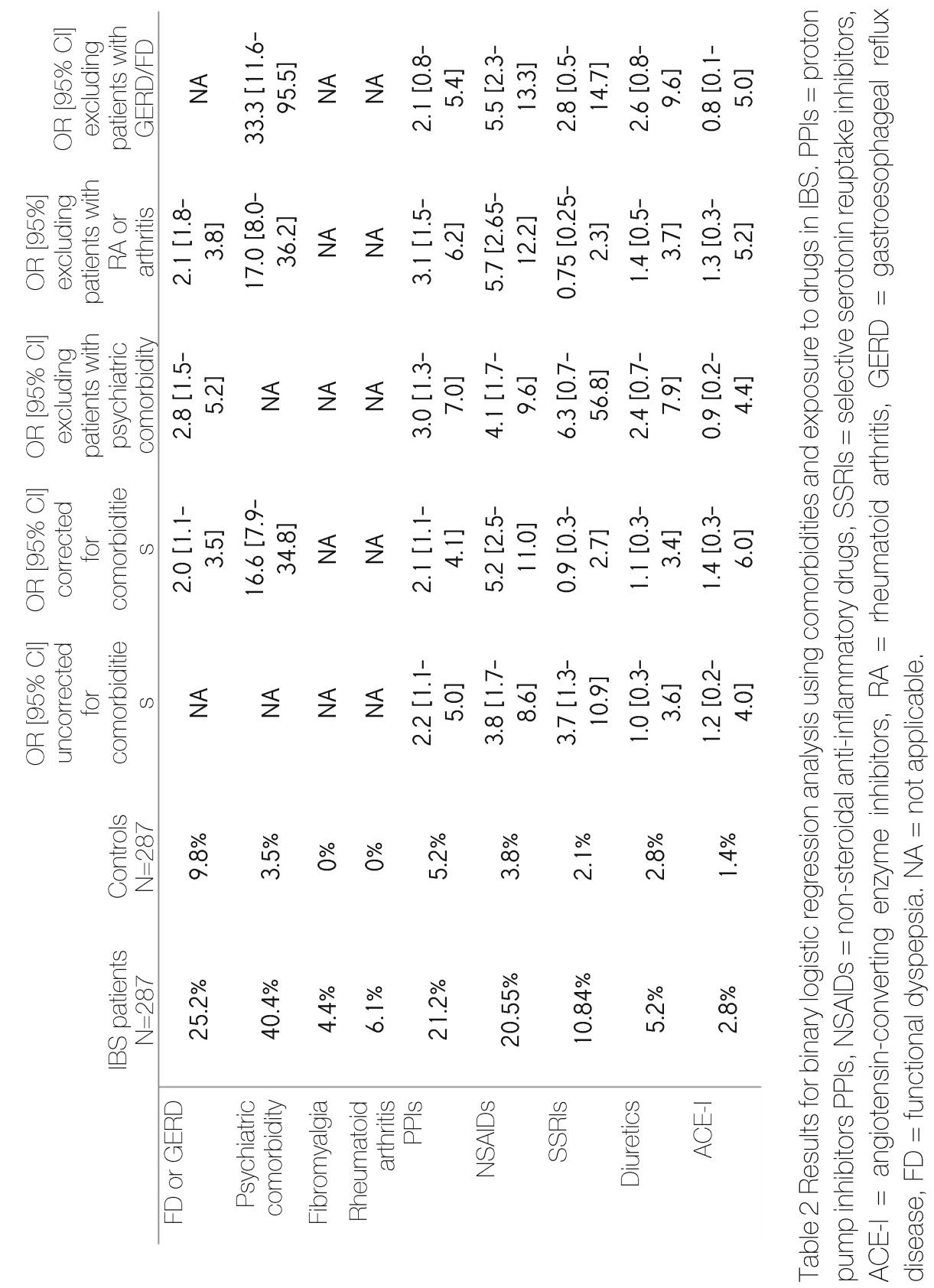




\begin{tabular}{r|ccc}
\multicolumn{3}{c}{ Type of PPI1 } & \multicolumn{3}{c}{ Type of NSAID2 } \\
\hline Pantoprazole & $44.0 \%$ & Diclofenac & $47.0 \%$ \\
Omeprazole & $28.8 \%$ & lbuprofen & $41.2 \%$ \\
Esomeprazole & $27.1 \%$ & Naproxen & $21.6 \%$ \\
Rabeprazole & $6.7 \%$ & Aceclofenac & $3.9 \%$
\end{tabular}

Table 3: Types of drugs used by irritable bowel syndrome patients. PPI = proton pump inhibitor. NSAID = non steroidal anti-inflammatory drug.

16 patients used multiple PPIs,

27 patients used multiple NSAIDs.

\subsection{Discussion}

In this study, we found that a significantly higher number of IBS patients use certain medications that can potentially alter intestinal homeostasis, such as PPIs and NSAIDs, in comparison to controls. Medication use of patients was examined in the period preceding the time of presentation at our clinic with their IBS symptoms, aiming to establish a temporal relationship between medication use and onset of symptoms.

We here demonstrate in our study population of IBS patients a positive association with IBS and exposure to PPIs and NSAIDs, but also to SSRIs. The former two are associated with alterations of intestinal physiology, whereas the latter is often prescribed in IBS due to concomitant psychiatric comorbidity. In order to delineate between a potential causal effect and mere therapeutic utilization of these drugs, we conducted analysis with the exclusion of IBS patients with certain comorbid conditions. For instance, IBS patients often suffer from functional dyspepsia; the degree of overlap varies between 15 and $42 \%$. 15,16 Because patients with IBS are more likely to have GERD and dyspepsia in comparison to controls, they are also more likely to receive PPI therapy. Therefore, following initial analyses, we conducted additional analyses excluding the cases and controls exposed to PPIs due to therapy for concomitant upper GI complaints based on functional dyspepsia or GERD. In this case, PPI exposure was not associated with IBS. We therefore presume 


\section{Chapter 11}

that the exposure to PPIs can be explained by the therapeutic use of PPIs for upper GI complaints.

We also found a positive association with the exposure to NSAIDs, reproducing previous literature findings. ${ }^{17}$ Pain-related symptoms of extragastrointestinal origin frequently observed in IBS could be the explanation for the increased use of NSAIDs in this population. ${ }^{18}$ Moreover, as IBS patients tend to suffer from recurring and therapyrefractory abdominal pain, overuse of NSAIDs in IBS patients can be extremely common in everyday clinical practice. Therefore, despite the fact that NSAIDs are known to alter intestinal physiology 19 and in particular barrier function, previous studies suggest that the association with NSAIDs is due to the tendency of patients with IBS having pain complaints rather than analgesics being a causative factor. ${ }^{17}$ We therefore conducted analyses with the exclusion of subjects with rheumatoid disorders, as a common source of extragastrointestinal pain. Results of these analyses still showed a significant association between NSAIDs use and IBS. This could be explained by the fact that patients were taking NSAIDs due to pain symptoms related to conditions other than rheumatoid disorders. On the other hand, recent findings have shown that NSAIDs can compromise intestinal permeability in IBS patients to a greater extent than in healthy subjects. ${ }^{3}$ Furthermore, another epidemiological study demonstrated that IBS patients using NSAIDs were also more likely to have persistent irritable bowel syndrome. ${ }^{20}$ This appears to be in line with the hypothesis that NSAID therapy affects intestinal permeability resulting in sustained low-grade mucosal inflammation. ${ }^{21}$ This implies that even if NSAIDs do not necessarily trigger IBS symptoms, they may be able to sustain the condition by altering intestinal physiology and in particular by impairing intestinal permeability.

PPIs and NSAIDs are often used simultaneously, with the former frequently co-prescribed to reduce gastrointestinal injury due to the latter. In our study, over a third of the patients using NSAIDs also used PPIs - presumably for gastroprotection. Recent video capsule studies suggest 22,23 a very high incidence (55-70\%) of intestinal damage in healthy humans taking both NSAIDs and PPIs for 2 weeks. A more recent study performed in rats 
demonstrated that PPIs lead to a marked exacerbation of small intestinal ulceration induced by NSAIDs, which was transferable to germ-free mice via microbiota isolated from the PPI-treated rats. This observation points to an important role for microbial alterations. When PPIs were administered alone, significant changes in intestinal microbiota were observed, with $80 \%$ reduction in the levels of the beneficial Bifidobacteria spp, whereas little effect was detected on the morphology of the intestinal mucosa. ${ }^{24}$

It is generally accepted that PPI therapy can alter intestinal microbial profiles by inducing hypochlorhydria resulting in a diminished host defense against certain bacteria. ${ }^{25-28} \mathrm{~A}$ recent study by Lombardo et al. indeed suggested that PPI therapy in humans may potentially result in small intestinal bacterial overgrowth (SIBO). ${ }^{13}$ It is not known whether the changes in intestinal microbiota induced by PPI therapy contribute to the development of symptoms and clinical conditions such as IBS. ${ }^{29}$ In another recent study using duodenal aspirates, no clear association was found between SIBO with IBS or PPI use. ${ }^{30}$ It therefore still remains unclear whether SIBO, if at all present in IBS, is a cause or merely an epiphenomenon of IBS, as microbial alterations most probably are not the sole explanation for symptom development in IBS. ${ }^{29}$ Overall, it is tempting to assume that PPIs can potentially induce alterations in intestinal microbiota, albeit not to a clinically significant degree, which can in turn impair the capacity of the intestine to respond to noxious agents, such as NSAIDs. Such 'two-hit' theory could provide an explanation for the relevance of co-exposure to PPIs and NSAIDs in the onset of IBS symptoms.

Besides PPIs and NSAIDs, the use of SSRIs was also associated with IBS in our study, but only when patients with psychiatric comorbidity were included in the analyses. Excluding IBS patients with psychiatric comorbidities from the analyses, accounting for $38 \%$ of our study population, resulted in a loss of positive association with exposure to SSRIs. This may be due to the high prevalence of psychiatric comorbidities in this population for which IBS patients receive SSRI therapy. This observation therefore suggests that the association of IBS and SSRI use is probably due to the therapeutic application of SSRIs for preexistent psychiatric conditions. Although no data were available on the duration of 


\section{Chapter 11}

psychiatric comorbid conditions, we postulate that these may have been present prior to the onset of the gastrointestinal symptoms and are therefore less likely to be involved in triggering IBS symptoms.

Although we were able to demonstrate a positive association with IBS and the use of drugs known to alter intestinal physiology, our study is hypothesis generating rather than proving an etiological relationship due to a number of limitations. While it is apparent that IBS patients utilize medication more often than controls, case-control studies generally do not allow interpretation with respect to a causal relation. In an attempt to establish a temporal relationship, we aimed to assess exposure to medication prior to onset of symptoms, implicating that this exposure can potentially trigger symptoms characteristic for IBS. However, we were not able to report on exact symptom history in the 180-day period investigated for drug exposure in relation to drug intake, which hinders the establishment of a true cause-effect relationship. We were also unable to report on a potential relationship between drug use and the severity of IBS-related symptoms. Furthermore, we could not take into account the use of over-the-counter medication. Also, our patient population is possibly a selected population consisting of patient presenting to our secondary/tertiary referral, which may not represent the IBS population as a whole.

\subsection{CONCLUSION}

Our case-control study points to an increased use of certain drugs prior to consultation for symptoms in IBS patients, in particular PPIs and NSAIDs. Both PPIs and NSAIDs are frequently prescribed drugs and IBS patients are prone to over-utilize drugs. Therefore, one should be aware that prescribing PPIs for upper GI complaints or NSAIDs for pain relief may potentially trigger mechanisms resulting in symptom generation representative for IBS. Animal data suggest that the combination of PPIs and NSAIDs, in particular, is able to induce profound alterations in intestinal homeostasis. As case-control studies generally do not allow interpretation of a cause-effect relationship, further research should include prospective evaluation of PPI users and NSAIDs users monitoring the 
development of IBS-symptoms in relation to drug exposure to ascertain whether this increased exposure to PPIs and NSAIDs should be considered as legitimate etiological factors in IBS. 


\subsection{REFERENCES}

1. Drossman DA, Camilleri M, Mayer EA, Whitehead WE. AGA technical review on irritable bowel syndrome. Gastroenterology 2002;123:2108-31.

2. Akehurst RL, Brazier JE, Mathers N, O'Keefe C, Kaltenthaler E, Morgan A, Platts M, Walters SJ. Health-related quality of life and cost impact of irritable bowel syndrome in a UK primary care setting. Pharmacoeconomics 2002;20:455-62.

3. Kerckhoffs AP, Akkermans LM, de Smet MB, Besselink MG, Hietbrink F, Bartelink IH, Busschers WB, Samsom M, Renooij W. Intestinal permeability in irritable bowel syndrome patients: effects of NSAIDs. Dig Dis Sci 2010;55:716-23.

4. Spiegel BM, Chey WD, Chang L. Bacterial overgrowth and irritable bowel syndrome: unifying hypothesis or a spurious consequence of proton pump inhibitors? Am J Gastroenterol 2008; 103:2972-6.

5. Facts and figures 2009. Volume 2010, 2010.

6. Forgacs I, Loganayagam A. Overprescribing proton pump inhibitors. BMJ 2008;336:2-3.

7. van Boxel OS, Hagenaars MP, Smout AJ, Siersema PD. Socio-demographic factors influence chronic proton pump inhibitor use by a large population in the Netherlands. Aliment Pharmacol Ther 2009;29:571-9.

8. Goettsch WG, van den Boom G, Breekveldt-Postma NS, Smout AJ, Herings RM. Treatment patterns and health care costs of mebeverine-treated IBS patients: a case-control study. Pharmacoepidemiol Drug Saf 2004;13:803-10.

9. Keszthelyi D, Jansen SV, Schouten GA, de Kort S, Scholtes B, Engels LG, Masclee AA. Proton pump inhibitor use is associated with an increased risk for microscopic colitis: a case-control study. Aliment Pharmacol Ther 2010;32:1124-8.

10. Limsui D, Pardi DS, Camilleri M, Loftus EV, Jr., Kammer PP, Tremaine WJ, Sandborn WJ. Symptomatic overlap between irritable bowel syndrome and microscopic colitis. Inflamm Bowel Dis 2007; 13:175-81.

11. Burgel N, Bojarski C, Mankertz J, Zeitz M, Fromm M, Schulzke JD. Mechanisms of diarrhea in collagenous colitis. Gastroenterology 2002;123:433-43.

12. Mullin JM, Valenzano MC, Whitby M, Lurie D, Schmidt JD, Jain V, Tully O, Kearney K, Lazowick D, Mercogliano G, Thornton JJ. Esomeprazole induces upper gastrointestinal tract transmucosal permeability increase. Aliment Pharmacol Ther 2008;28:1317-25.

13. Lombardo L, Foti M, Ruggia O, Chiecchio A. Increased incidence of small intestinal bacterial overgrowth during proton pump inhibitor therapy. Clin Gastroenterol Hepatol 2010;8:504-8.

14. Ulluwishewa D, Anderson RC, McNabb WC, Moughan PJ, Wells JM, Roy NC. Regulation of tight junction permeability by intestinal bacteria and dietary components. J Nutr 2011;141:769-76.

15. Talley NJ, Dennis EH, Schettler-Duncan VA, Lacy BE, Olden KW, Crowell MD. Overlapping upper and lower gastrointestinal symptoms in irritable bowel syndrome patients with constipation or diarrhea. Am J Gastroenterol 2003;98:2454-9. 
16. Ford AC, Marwaha A, Lim A, Moayyedi P. Systematic review and meta-analysis of the prevalence of irritable bowel syndrome in individuals with dyspepsia. Clin Gastroenterol Hepatol 2010;8:401-9.

17. Locke GR, 3rd, Zinsmeister AR, Talley NJ, Fett SL, Melton LJ. Risk factors for irritable bowel syndrome: role of analgesics and food sensitivities. Am $\mathrm{J}$ Gastroenterol 2000;95:157-65.

18. Talley NJ, Zinsmeister AR, Melton LJ, 3rd. Irritable bowel syndrome in a community: symptom subgroups, risk factors, and health care utilization. Am J Epidemiol 1995; 142:76-83.

19. Bjarnason I, MacPherson A, Hollander D. Intestinal permeability: an overview. Gastroenterology 1995;108:1566-81.

20. Kalantar JS, Locke GR, 3rd, Talley NJ, Zinsmeister AR, Fett SL, Melton LJ, 3rd. Is irritable bowel syndrome more likely to be persistent in those with relatives who suffer from gastrointestinal symptoms? A population-based study at three time points. Aliment Pharmacol Ther 2003;17:1389-97.

21. Bjarnason I, Hayllar J, MacPherson AJ, Russell AS. Side effects of nonsteroidal antiinflammatory drugs on the small and large intestine in humans. Gastroenterology 1993; 104:1832-47.

22. Maiden L, Thjodleifsson B, Theodors A, Gonzalez J, Bjarnason I. A quantitative analysis of NSAID-induced small bowel pathology by capsule enteroscopy. Gastroenterology 2005; 128:1172-8.

23. Fujimori S, Gudis K, Takahashi Y, Seo T, Yamada Y, Ehara A, Kobayashi T, Mitsui K, Yonezawa M, Tanaka S, Tatsuguchi A, Sakamoto C. Distribution of small intestinal mucosal injuries as a result of NSAID administration. Eur J Clin Invest 2010;40:50410.

24. Wallace JL, Syer S, Denou E, de Palma G, Vong L, McKnight W, Jury J, Bolla M, Bercik P, Collins SM, Verdu E, Ongini E. Proton pump inhibitors exacerbate NSAIDinduced small intestinal injury by inducing dysbiosis. Gastroenterology 2011;141:1314-22, 1322 e 1-5.

25. Lewis SJ, Franco S, Young G, O'Keefe SJ. Altered bowel function and duodenal bacterial overgrowth in patients treated with omeprazole. Aliment Pharmacol Ther 1996; 10:557-61.

26. Williams C, McColl KE. Review article: proton pump inhibitors and bacterial overgrowth. Aliment Pharmacol Ther 2006;23:3-10.

27. Thorens J, Froehlich F, Schwizer W, Saraga E, Bille J, Gyr K, Duroux P, Nicolet M, Pignatelli B, Blum AL, Gonvers JJ, Fried M. Bacterial overgrowth during treatment with omeprazole compared with cimetidine: a prospective randomised double blind study. Gut 1996;39:54-9.

28. Fried M, Siegrist H, Frei R, Froehlich F, Duroux P, Thorens J, Blum A, Bille J, Gonvers JJ, Gyr K. Duodenal bacterial overgrowth during treatment in outpatients with omeprazole. Gut 1994;35:23-6.

29. Spiegel BM. Questioning the bacterial overgrowth hypothesis of irritable bowel syndrome: an epidemiologic and evolutionary perspective. Clin Gastroenterol Hepatol 2011;9:461-9; quiz e59. 
30. Choung RS, Ruff KC, Malhotra A, Herrick L, Locke GR, 3rd, Harmsen WS, Zinsmeister AR, Talley NJ, Saito YA. Clinical predictors of small intestinal bacterial overgrowth by duodenal aspirate culture. Aliment Pharmacol Ther 2011;33:1059-67. 
Drug exposure in IBS 
General discussion

D. Keszthelyi

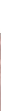


Part IV. Intestinal barrier function: effect of drugs in disease development

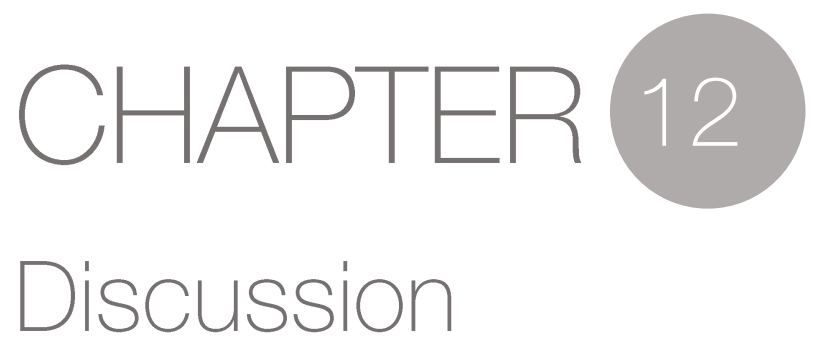

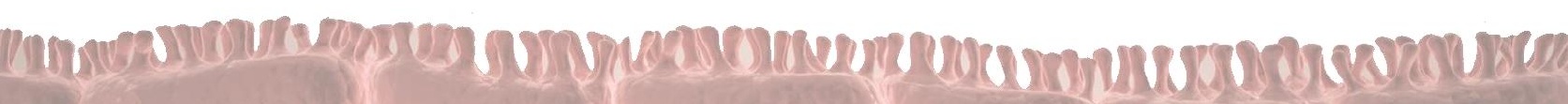




\subsection{THE INTESTINAL BARRIER FUNCTION: THE KEY TO HOMEOSTATIO CONTROL}

'All Disease Begins in the Gut' - a statement, which has been attributed to Hippocrates and was allegedly made over 2000 years ago. It is needless to say that many would consider such hypothesis to be an oversimplification of pathophysiology. Nevertheless, accumulating evidence suggests an important role for a dysregulation in intestinal barrier function in a wide array of chronic inflammatory or degenerative disease in many organs systems, including the heart (chronic heart failure) ${ }^{1}$, airways (bronchial asthma, ${ }^{2}$ chronic obstructive pulmonary disease), ${ }^{3}$ locomotor apparatus (rheumatoid disorders), ${ }^{4}$ skin (dermatitis herpetiformis), ${ }^{5}$ pancreas (type 1 diabetes), ${ }^{6}$ liver (non-alcoholic fatty liver disease), ${ }^{7}$ the intestine (inflammatory bowel disease, ${ }^{8}$ celiac disease ${ }^{9}$ ) but also obesity. ${ }^{10}$

In the past few decades, the concept of 'the leaky gut' has received increased attention in both layman media as well as in the scientific community. More importantly, current medical approaches focus more and more on aiming to maintain and preserve optimal health and overall well-being in individuals, with particular emphasis on gut health through optimal diet, of which barrier function is a cardinal element.

In terms of maintenance of homeostasis, continuous interaction between the milieu intérieur, as first described by Claude Bernard, and the external environment is an important determinant of physiological and behavioral adaptation to external stimuli. This interaction is anatomically mainly localized to the epithelia of skin, airways and the gastrointestinal tract. Of these three interfaces, the gastrointestinal tract has by far the largest surface area (almost 200 square meters, some 100 times the surface area of the exterior of the body) and is the only epithelial surface, in principle, that interacts with substances that are orally ingested.

This unique characteristic of the gut necessitates a selective discriminatory function, which enables adequate distinction between beneficial and potentially noxious luminal elements. A malfunction in this discriminatory capacity can result in inadequate 
physiological intestinal responses such as a failure to take up nutrients or a facilitation of entrance of pathogens or toxic agents. ${ }^{2}$

\section{THE INTESTINAL EPITHELIUM: IMPORTANT SENSORY FUNCTION}

Interaction of the gastrointestinal epithelium with luminal antigens generates a number of physiological responses, including sensory information. In this respect, enterochromaffin cells appear to play an important role in serving as sensory transducers. Enterochromaffin cells are a specialized type of epithelial cells, which are able to synthesize a number of signaling molecules, including serotonin. Certain luminal antigens (chemical stimuli) but also mechanical stimuli can activate these enterochromaffin cells to release serotonin from the basolateral membrane. This released serotonin triggers mucosal nerve endings of enteric, spinal or vagal nerve cells, generating a sensory signal. Furness and Clerc $^{11}$ refer to this as 'neural message.' In addition, these authors also distinguish two other functions of the epithelium: an immune and endocrine function. The immune function, mainly dependent on M-cells, mucosal dendritic cells and other immunocytes, is important in terms of determining the adequate reaction to certain luminal antigens (tolerance or defense type immune reaction). The endocrine function relates to the great number of hormones and peptides secreted by enteroendocrine cells in response to certain nutrients, including cholecystokinin, peptide YY (PYY), glucagonlike peptide 1 (GLP-1), which play a cardinal role in signaling satiety.

\section{PARACRINE SIGNALING FUNCTION OF THE EPITHELIUM}

Based on the findings in this thesis, we postulate that the epithelium has an additional function, which can be termed as 'paracrine message' in the line of Furness and Clerc (see Figure 1). ${ }^{11}$ In the study described in chapter 8 , we have demonstrated that released serotonin is able to reinforce intestinal barrier function. We hypothesized that this phenomenon may in fact represent a protective mechanism related to increased serotonergic signaling in the mucosa, presumably originating from enterochromaffin cells, to prevent penetration of potentially noxious luminal substances into the lamina propria. A more profound understanding of this proactive mechanism would allow the 


\section{Chapter 12}

development of therapeutic strategies specifically aimed at the reinforcement of the intestinal barrier against potentially noxious substances.

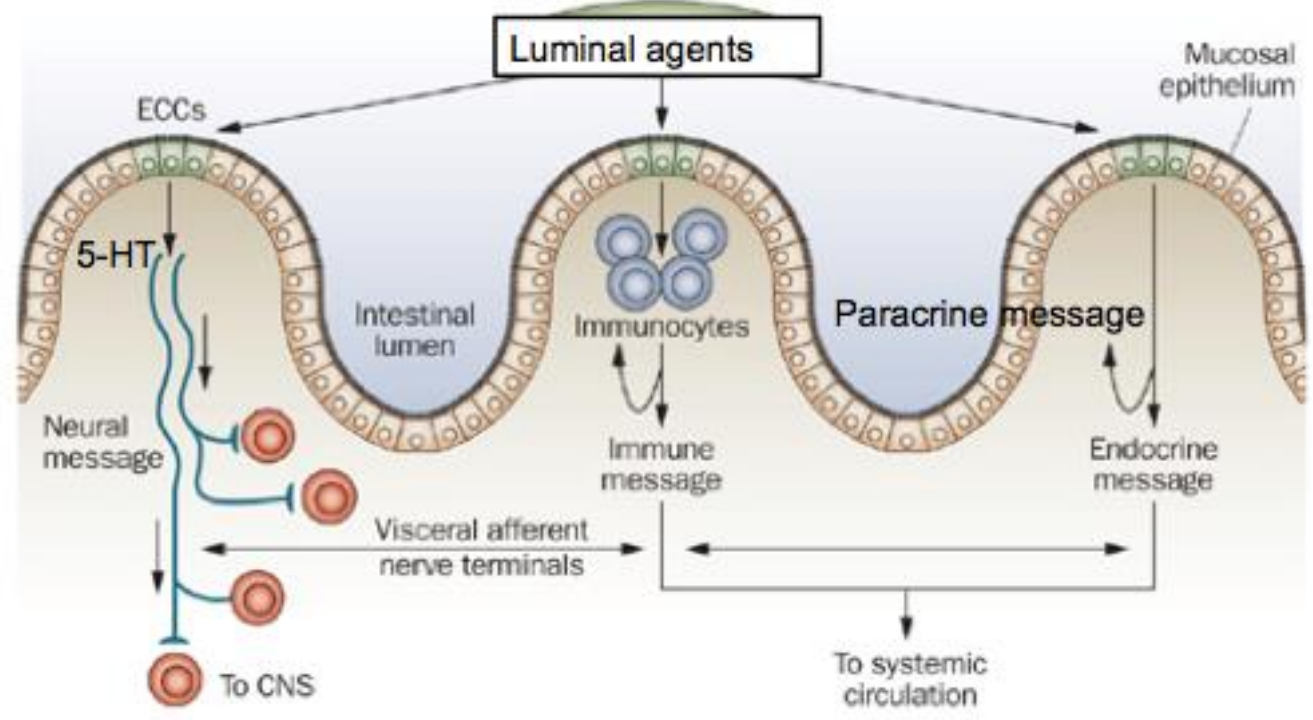

Figure 1: Schematic figure representing functions of the intestinal epithelium (neural, endocrine, immune and paracrine). Adapted from Furness and Clerc (ref 11.)

Previous work by Pai and Horseman ${ }^{12}$ performed in cell cultures of mammary epithelial cells suggest the involvement of the $5-\mathrm{HT}_{7}$ receptor located on the basolateral side of epithelial cells in the regulation of tight junctions. Activation of the $5-\mathrm{HT}_{7}$ receptor has been shown to promote intestinal integrity through protein kinase $A$ (PKA) activation. ${ }^{12}$ Future work will need to identity which molecules and pathways are involved in this serotonergic-modulated increase in barrier function in the intestinal epithelium. Assuming 5- $\mathrm{HT}_{7}$ receptors are at least in part involved in this mechanism, stimulation of these receptors could offer an attractive intervention to reinforce intestinal barrier function.

This thesis has provided evidence for another unique characteristic of the intestinal epithelium aimed at maintenance of homeostasis. In chapter 3, we describe a study in which we used acute tryptophan depletion to influence mucosal serotonergic mechanism 
by altering precursor availability. Mucosal serotonin metabolism, as illustrated above, is paramount in terms of orchestration of intestinal function. Quite interestingly, in that study we demonstrated that the machinery responsible for maintaining serotonergic homeostasis in the intestinal mucosa has the capacity to compensate for an acute decrease in the availability of its precursor, tryptophan. Absence of such mechanism would potentially result in derailment of intestinal function following the ingestion of food poor in tryptophan, which would be biologically highly undesirable. Such mechanism seems, therefore, exceptionally relevant given the important signaling function of serotonin in the intestinal mucosa.

\subsection{NOVEL INSIGHT INTO THE PATHOGENESIS OF VISCERAL HYPERSENSITIVITY IN IBS: THE ROLE FOR SEROTONIN-MEDIATED INTESTINAL BARRIER FUNCTION}

The gastrointestinal tract, under normal physiological conditions, is not a source of conscious sensory experiences apart from registration of physiological sensations such as fullness, satiety, hunger and urgency. Conscious sensations of abdominal pain, cramps and bloating are therefore considered abnormal. Nearly all individuals will experience such pathological sensations during a lifetime. Experiencing such unpleasant sensations not related to specific disease condition on a regular basis refers to functional disorders, among them irritable bowel syndrome (IBS).

According to epidemiological data, one to two in every ten people in the Western world is affected by IBS, i.e. suffers from abdominal pain and/or discomfort for over 3 months accompanied by an alteration of bowel habits, as diagnosed by the Rome III criteria, in the absence of organic disease. ${ }^{13}$ One third of this population is bothered by symptoms to such degree that results in consulting a general physician or hospital physician. ${ }^{13,14}$ Up to $60 \%$ of IBS patients also exhibit increased sensory responses to mechanical distension of a hollow organ, commonly referred to as visceral hypersensitivity. Visceral hypersensitivity is therefore considered by many clinicians and scientists as a biological 


\section{Chapter 12}

'hallmark' of IBS. ${ }^{15}$ Furthermore, visceral hypersensitivity is believed to be an important contributing factor to pain symptom generation in IBS. ${ }^{16}$

The following questions therefore arise:

$>$ 1. Assuming that exposure to exogenous (luminal) antigens is similar in a given population, why do only certain individuals develop IBS?

> 2. What are the reasons that only about half of the IBS patients exhibit visceral hypersensitivity?

This thesis has sought the answers to these questions. In the past few decades, a number of hypotheses have been formulated on the pathogenesis of IBS. In this thesis, we provide evidence in support of the hypothesis that considers impaired barrier function as the key pathogenetic factor in IBS. In chapter 8, we demonstrate that IBS patients, compared to healthy controls, have impaired barrier function due to a primary defect in the expression of tight junction proteins. A number of studies have previously investigated the possible explanations for this defect. First, impaired barrier function in IBS has been related to increased luminal protease activity. ${ }^{17,18}$ This has been shown, on one hand, to result in activation of protease activated receptor 2 (PAR-2), which can lead to increased intestinal permeability. ${ }^{19}$ Serine proteases, such as pancreatic tryptase, are potent activators of PAR-2 receptors.20 On the other hand, cysteine proteases, found at increased concentration in fecal samples of constipation-predominant IBS patients, have been demonstrated to degrade occludin, a key transmembrane component of tight junctions, resulting in impairment of barrier function. ${ }^{21}$ It has been suggested that mast cells and microbiota constitute potential sources of proteases affecting barrier function. ${ }^{18,22}$

Another theory suggests that increased proteasomic degradation ${ }^{23}$ and/or internalization of tight junction proteins, in particular occludin, ${ }^{24}$ are the mechanism behind impairment of the barrier function in IBS. 
In this thesis, we provided further evidence for an intracellular maldistribution of occludin in the duodenal mucosa of IBS patients, which possibly reflects occludin internalization. Furthermore, we demonstrated in IBS patients a failure to reinforce the intestinal barrier following increased serotonergic signaling. In other words, in case the intestinal barrier is defect, built-in protective mechanisms will also fail to reinforce this barrier. Strategies in these patients with increased intestinal permeability will need to be designed and developed aiming at restoring the defects by eliminating factors leading to increased tight junction protein breakdown (i.e. protease inhibition) or malicious intracellular redistribution.

Our currently rapidly increasing understanding of tight junction physiology can also provide an elegant explanation for the fact that symptoms, in particular pain, predominantly occur predominantly in the post-prandial setting in IBS patients. Nutrient uptake, as described in the introduction of this thesis, leads to physiological and functionally relevant increments in intestinal permeability, facilitating rapid nutrient absorption. In case the intestinal barrier is defect, such physiological post-prandial increase in permeability may be abnormal in intensity or duration and lead to increased passage of noxious molecules able to trigger nerve endings to generate pain. This increased influx of luminal substances can hence result in increased pain symptom generation by excitation of visceral afferents. Such phenomenon could be defined as 'increased or nociceptive nutrient sensing.' On the other hand, postprandial increase in pain symptoms may also be independent of increased intestinal permeability, for instance due to hypervigilance and anticipatory fear of developing symptoms following food intake. Conversely, as pointed out in the introduction of this thesis, stress is known to increase intestinal permeability, allowing the generation of a vicious circle. More comprehensive analysis of neurobiological responses, and in particular pain processing following nutritional stimulation could allow further pathophysiological characterization of such underlying mechanisms. The availability of high resolution brain imaging techniques offers an unparalleled opportunity to investigate these mechanisms. 
Pain symptoms in IBS are assumed to be related to visceral hypersensitivity. Seeking the potential causes of visceral hypersensitivity in IBS is as complex as the pathophysiology of IBS itself. Current hypotheses consider visceral hypersensitivity as being the result of alterations in both peripheral and central nociceptive processes. ${ }^{25}$ As pain is a conscious sensation, afferent signals originating in the intestinal mucosa need to reach the neocortex and pass several levels of nociceptive processing, including the spinal cord, the brainstem and the thalamus.

Accordingly, visceral hypersensitivity can either originate from increased peripheral discharge of visceral afferents or from central augmentation of peripheral input. In this thesis, we investigated two potential mechanisms for peripheral nociceptive sensitization:

> 1. Impaired intestinal barrier function allows the passage of luminal substances that have the potential to excite visceral afferents, which would normally not occur (or at least not occur to a more pronounced, pathological extent) in case the barrier is intact. Such stimulation of visceral afferents may result in increased peripheral discharge and subsequent pain symptom generation. In this thesis, we have provided evidence that increased nociceptive response to mechanical stimuli in IBS is associated with an increased intestinal permeability (chapter 8).

> 2. Visceral afferents can become sensitized to nociceptive stimuli, for instance following inflammation, i.e. activation can occur at a lower stimulation threshold or the intensity of nociceptive signaling can be increased. ${ }^{26}$ Such sensitization can persist even when the initial mucosal inflammation has ceded. We provide evidence for this postulate in chapter 7 , in which we show that transcription of the pain signaling molecule TRPV 1 is increased in mucosal samples of IBS patients and that this correlates with pain symptom severity. Increased expression of TRPV1 receptor on mucosal nerve endings allows mucosal afferents to be excited by stimuli that normally do not cause pain (allodynia) or cause increased nociceptive signaling following painful stimuli (hyperalgesia). Such sustained alterations in the mucosal 
neurobiology can also provide an explanation for the chronic and recurrent nature of pain complaints in IBS patients.

In this thesis, we also investigated why merely a certain proportion instead of all IBS patients become hypersensitive. Interestingly, we found that only hypersensitive patients reacted to a serotonergic challenge with a pro-nociceptive response, even though both normo- and hypersensitive IBS patients had impaired barrier function (see chapter 8). This suggests that a disturbance in serotonergic signaling has an important role in the development of augmented sensory responses in hypersensitive patients.

Remarkably, a number of healthy subjects also became manifestly hypersensitive upon interference with their serotonergic metabolism. This group could in fact represent a 'hypersensitivity prone' group of healthy individuals. Taken together, these data suggest a role for serotonin, in addition to an impaired barrier function, in the development of visceral hypersensitivity.

\section{SEROTONERGIC INTERVENTIONS: DIFFERENTIAL EFFECTS ON VISCERAL PERCEPTION}

As pointed out previously, the pathogenesis of visceral hypersensitivity in IBS is influenced by peripheral and central mechanisms. This also holds true for serotonergic metabolism.

The role of serotonin in visceral perception in IBS has been previously investigated employing the acute tryptophan depletion (ATD) method, which we also used in this thesis to investigate its effects on mucosal serotonin metabolism (chapter 3). Kilkens et al. found that ATD, aimed to decrease serotonergic metabolism, significantly decreased pain perceptual thresholds to rectal distension in IBS-D patients. ${ }^{27}$ In addition, treatment with ATD was associated with increased overall pain scores compared to placebo. These observations appear to be in contrast with findings in this thesis, i.e. that positive modulation of serotonergic modulation by 5 -HTP also increases pain perception during rectal distension. 


\section{Chapter 12}

How is it possible that positive and negative modulation of serotonergic modulation both result in increased nociception? We believe that this apparent discrepancy can be explained as follows. Decreasing tryptophan availability by ATD has been demonstrated to decrease brain $5-\mathrm{HT}$ synthesis throughout much of the cerebral cortex in healthy volunteers. ${ }^{28}$ Furthermore, a recent study by Labus et al. showed that in healthy controls, ATD causes a disinhibition of the entire emotional arousal network during rectal distension. ${ }^{29}$ This loss of negative inhibition can lead to altered processing of visceral afferent signals. In support of this observation, animal studies have also shown that an acute decrease in central serotonergic activity is associated with increased sensory information processing. ${ }^{30}$ Kilkens et al. also described that ATD induces a depression-like affective memory bias in IBS patients. ${ }^{27}$ Furthermore, the effect of ATD treatment on rectal perception was not pressure-dependent and mechanoelastic characteristics of the rectum were not affected by ATD. In chapter 3 of this thesis, we demonstrate that ATD does not induce alterations in mucosal serotonin metabolism. It is therefore tempting to assume that the increase in visceral perception by ATD is primarily caused by changes induced in the central nervous system.

Administration of 5-HTP, on the other hand, appears to have an effect on mucosal serotonin metabolism, as shown in chapter 8 . We postulate that increase in mucosal serotonergic metabolism induces increased afferent nociceptive discharge resulting in increased perception, as discussed in chapter 8 . Therefore, the discrepancy with regards to viscero-nociceptive response following ATD and 5-HTP administration is related to the different, functionally distinct serotonergic systems (central vs. mucosal) primarily affected by the intervention. This way, negative modulation of the central serotonergic system through ATD as well as the positive modulation of the mucosal serotonergic system both result in increased visceral perception. 
INTEGRATIVE HYPOTHESIS ON VISCERAL HYPERSENSITIVITY: MODULATORY BALANCE OF NOCICEPTIVE PROCESSING

Central nociceptive mechanisms appear to have profound influence on pain processing. Accordingly, peripheral and/or central sensitization alters the perception of peripheral sensory input from the gastrointestinal tract and summation of peripheral sensory input and central modulatory processing determines the overall sensory perception of an individual. Alterations in the modulatory balance of pro- and anti-nociceptive central processing of peripheral input have received increasing attention in serving as an integrative mechanism for visceral hypersensitivity. This hypothesis could also provide another explanation for the fact that not all IBS patients exhibit this hypersensitivity: intact central (spinal and/or cortical) nociceptive control has the capacity to suppress noxious peripheral input from afferents, regardless whether these are sensitized, hereby ensuring that these sensations remain below a conscious level.

Novel integrative approaches, in which both central and peripheral nociception can be examined, could possibly provide a more detailed understanding of pain symptom generation in IBS, which will allow the development of effective analgesic therapeutic entities.

\section{RECTAL BALLOON DISTENSIONS: A LEGITIMATE MARKER FOR A PERIPHERAL CONTRIBUTION TO VISCERAL HYPERSENSITIVITY?}

In chapters 5 and 6 , we describe extensively the scientific and diagnostic value of rectal mechanical balloon distension in IBS, commonly known as the barostat method. Despite the fact that not all patients exhibit hypersensitivity to rectal distensions, the barostat has been shown by several studies to be a reliable, reproducible and valid approach to assess visceral perception (chapter 6).

However, a number of recent observations have implicated that subjective reporting (for instance using visual analogue scale scores) during rectal distension can largely be influenced by psychological factors, such as vigilance and anxiety - again representing 


\section{Chapter 12}

the importance of central modulatory effects in visceral perception. IBS patients have in fact been shown to have a psychological tendency to report urge and pain rather than increased neurosensory sensitivity. ${ }^{31}$ This makes the identification of patients who have true visceral hypersensitivity, without the interference of subjective reporting, especially challenging. This is particularly true in a patient group with a high incidence (50-80\%) of psychological disorders such as heightened anxiety, depression, somatization, dysthymia, and panic disorders. ${ }^{13}$

Another important challenge is the observation that responses to rectal balloon distension only poorly correlate to symptoms, ${ }^{32}$ i.e. the patients who are most hypersensitive to balloon distension are not necessarily those who experience the highest intensity of chronic abdominal pain symptoms in daily life. Further optimization and standardization of test protocols and conditions might overcome these challenges.

In this respect, there are a number of relevant considerations.

> 1. A key theoretical consideration is that visceral pain can occur even in the absence of an adequate peripheral stimulus. ${ }^{33}$ Alternatively, the same brain areas can be activated by administration of a certain stimulus, as well as by anticipation of the stimulus itself. Furthermore, patients generally experience chronic pain, which cannot be related to ongoing luminal distensions at pressures exceeding $50 \mathrm{mmHg}^{34}$ Therefore, attempts should be made to take into account emotional-cognitive influences on perceptive responses and hypervigilance and anticipatory anxiety should be minimized during testing.

> 2. Distension protocols can be designed to specifically stimulate serosal, i.e. high threshold visceral afferents. The latter appears to be particularly interesting as recent evidence suggests that these afferents are sensitized in IBS and are responsible for increased peripheral discharge contributing to visceral hypersensitivity (chapter 6). Therefore, selective stimulation of these afferents may identify an evident peripheral component in visceral hypersensivity. 
> 3. A major technical consideration with respect to the relevance of the rectal distension method for symptom generation is the fact that mechanical distension of the rectum is a rather a-physiological stimulus. It appears highly unlikely that chronic pain generation in IBS is related to supraphysiological pressures due to distension of the rectum. However, it is generally accepted that hypersensitivity to mechanical distension when measured in the rectum is a characteristic of the entire intestinal tract in IBS. On the other hand, considering the fact that pain symptoms in IBS often occur postprandially, chemical (nutrient) stimulation of the small intestine with subsequent recordings of the physiological responses (by verbal rating, brain imaging and/or neurophysiological methods) appears to provide an additional and more complete understanding of pain symptom generation in IBS.

\section{PLASMA 5-HIAA: A POTENTIAL NOVEL BIOMARKER FOR VISCERAL HYPERSENSITIVTY}

Several research groups have attempted to identify a biomarker for IBS and in particular visceral hypersensitivity. In this thesis, we provide preliminary evidence for 5-HIAA, the main metabolic breakdowm product of 5-HT, as a marker for visceral hypersensitivity: hypersensitive IBS patients demonstrate increased baseline plasma levels of 5-HIAA compared to normosensitive IBS patients or healthy controls. 5-HIAA is produced primarily in the gut and is believed therefore to reflect intestinal serotonin metabolism. An analytical advantage of $5-$ HIAA is that it is not stored by platelets, like serotonin, therefore, destruction of platelets during sample processing does not influence 5-HIAA levels (provided the analysis is conducted in the presence of a reductive substance, such as ascorbic acid, preventing oxidative breakdown of serotonin). Measurement of fasting plasma 5-HIAA may therefore easily identify hypersensitive IBS patients. Furthermore, taking a single fasting plasma sample represents considerable less test burden compared to introduction and distension of a rectal balloon. However, given the small number of patients examined in this thesis, further, larger scale studies will be needed to establish whether plasma 5-HIAA will prove to be a legitimate biomarker of hypersensitivity. 


\section{FUTURE DIRECTIONS FOR IBS THERAPY}

Findings described in this thesis add to a body of evidence suggesting that impairment of the intestinal barrier plays a key role in the pathogenesis of IBS. It appears that this impairment is characteristic to both normo- en hypersensitive IBS patients. In addition, serotonergic signaling plays an important role in the generation of pro-nociceptive signals resulting in visceral hypersensitivity.

Restoration of the intestinal barrier therefore offers a tempting alternative to counteract pathophysiological processes contributing to the development of IBS and visceral hypersensitivity in particular. Assuming that proteases play a role in the impairment of the intestinal barrier, therapeutic approaches could be aimed at decreasing luminal protease activity. For instance, administration of a certain soy-derived ingredient has been demonstrated to prevent stress-induced increase in intestinal permeability and visceral hypersensitivty in rats. This effect was shown to be related to inhibition of luminal proteolytic activity and in increase in tight junction protein expression. ${ }^{35}$

Also, elimination of certain dietary components can prevent damage to the intestinal barrier. Dietary modification, through for instance the avoidance of gluten, known to impair barrier function, has been suggested to be highly effective in a substantial group of IBS patients. ${ }^{36} 37$ Such nutritional therapeutic entities, acting through enhancement of the intestinal barrier function, appear to be very promising and may open a new horizon in the treatment of irritable bowel syndrome.

\subsection{POTENTIAL ROLE FOR DRUGS IN THE PATHOGENESIS OF GASTROINTESTINAL DISORDERS}

Drugs are an important group of luminal agents able to affect the intestinal barrier. Nonsteroidal anti-inflammatory drugs (NSAIDs) have long been known to increase intestinal permeability. The question therefore arises, whether drug expose, in fact, plays a role in 
the development of certain gastrointestinal disorders by virtue of impairing the intestinal barrier.

We conducted a systemic review and a case-control study to investigate the potential pathogenetic role of drugs, in particular NSAIDs and proton pump inhibitors (PPIS) in two distinct populations: IBS patients and patients with microscopic colitis. Similarly to IBS, patient with microscopic colitis are characterized by increased intestinal permeability. In our case-control studies, we found an increased use of NSAIDs and PPIs in both patient groups. This led us to hypothesize that exposure to certain drugs may trigger certain mechanisms, potentially through alterations in barrier function, which may lead to symptom generation. It is however important to note, that associations found in casecontrol studies not necessarily are reflecting a cause-effect relationship. Furthermore, given the wide use of these drugs among the general population, we believe that such association most probably simply reflects an unfortunate idiosyncratic reaction to the particular drug in question (chapter 9).

Our preliminary experimental data suggest, however, that the PPI esomeprazole has direct effects on intestinal epithelial cells: esomeprazol treatment was found to increase transepithelial electrical resistance in vitro in Caco-2 cells (see Figure 2, unpublished data). This may reflect an inhibitory affect on ion transport across the epithelial cell layer. Further studies will be necessary to ascertain whether this direct effect on epithelial cells has any relevance to influence intestinal permeability and disease development.

Besides affecting ion transport and electrolyte balance, PPIs are known to be able to cause profound alterations in intestinal homeostasis. Figure 3 shows a hypothetic overview of potential mechanisms by which PPIs carry the capacity to impair intestinal barrier function. 


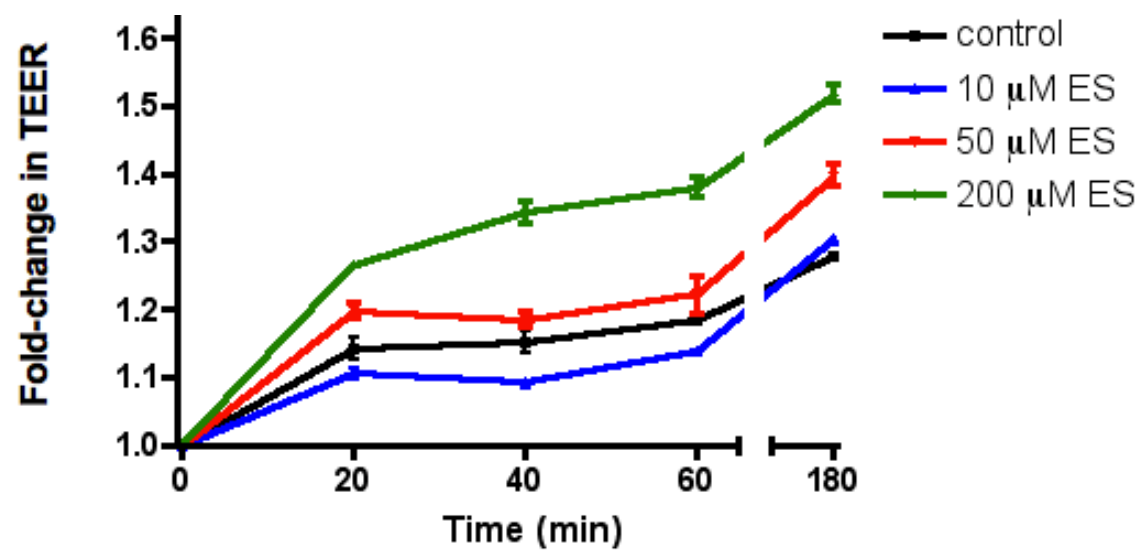

Figure 2: Effect of esomeprazole treatment on the transepithelial electrical resistance of Caco-2 monolayers. Data expressed as mean change compared to baseline measurement_SEM. ES= esomeprazole. TEER = transepithelial electrical resistance.

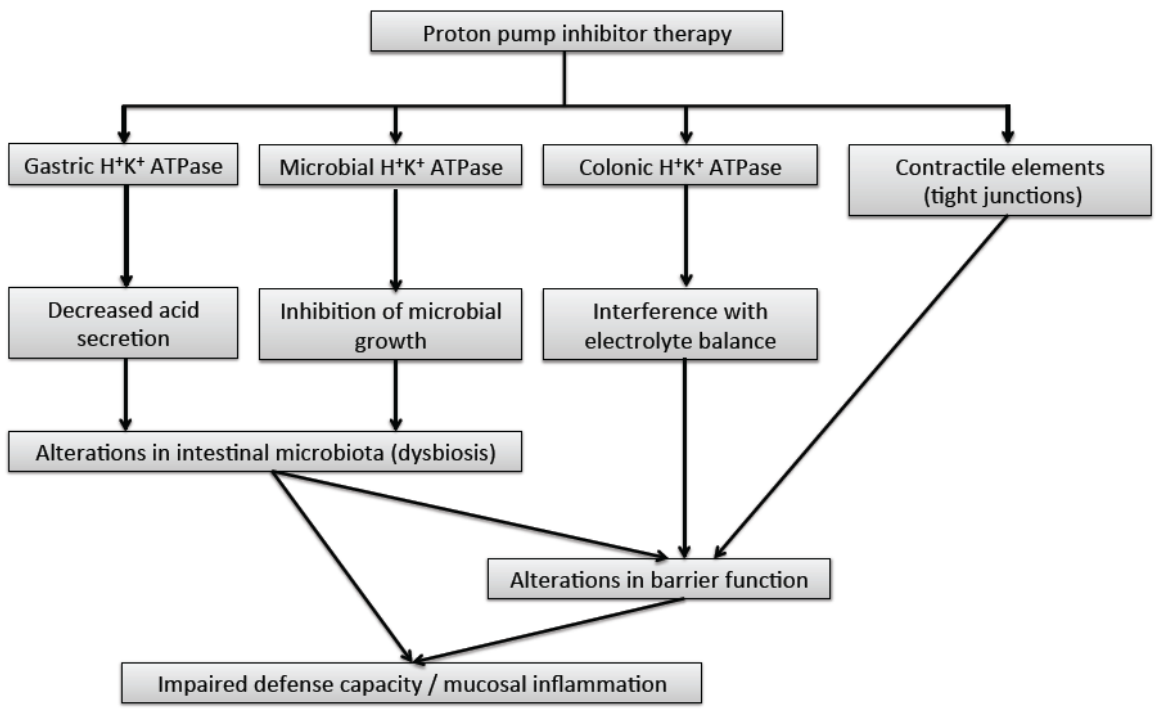

Figure 3: Schematic overview of potential mechanisms involved in proton pump inhibitorinduced increase in intestinal permeability.

With regard to the pathogenetic role of drugs, and in particular PPIs, we have formulated a 'two-hit' hypothesis. One might postulate that PPIs may induce alterations in intestinal microbiota and/or in intestinal barrier function, albeit not to a clinically significant degree, 
which can in turn impair the capacity of the intestine to respond to potentially noxious luminal agents, which thereby can provide the 'second hit' and lead to gastrointestinal disorders. By virtue of interfering with intestinal homeostasis, PPIs may potentially initiate or exacerbate ongoing unfavorable mucosal immune activation. Such PPI-induced impairment in defense capacity, provided there is a genetic susceptibility present, may therefore lead to the development of a manifest clinical condition. ${ }^{38}$

Given the idiosyncratic nature of drug-induced microscopic colitis (see chapter 9), we consider pharmacogenetic factors to be important determinants in such conditions. Cytochrome P450 (CYP) 2C19 mediates the major metabolic transformations of the PPIs omeprazole, pantoprazole, lansoprazole and esomeprazole. Genetic polymorphism of CYP2C19 can lead to significant phenotypic variation in the activity of this isoenzyme and thus in the metabolism of PPIs. Therefore, CYP2C19 genotyping may be warranted in PPI-related adverse reactions, such as microscopic colitis. ${ }^{39}$

Most probably however, considering the wide use of drugs such as PPIs and NSAIDs in the general population, exposure to these drugs is not the sole factor involved in the pathogenesis of either IBS or microscopic colitis. On the other hand, it is important to recognize that patients taking these drugs may warrant investigation for $\mathrm{MC}$ when developing watery diarrhea. It is crucial to ascertain the temporal relationship between exposure and symptom onset to support a causative role. After diagnosis, attempt should be made to discontinue the suspected drug.

Future studies will need to ascertain the exact pathomechanistic role of these drugs in gastrointestinal disorders and whether this is related to an effect on intestinal barrier function. In particular, further research should also include prospective evaluation of PPI users and NSAIDs users monitoring the development of gastrointestinal symptoms in relation to drug exposure to ascertain whether this increased exposure to PPIs and NSAIDs should be considered as legitimate etiological factors in IBS. 


\subsection{REFERENCES}

1. Sandek A, Anker SD, von Haehling S. The gut and intestinal bacteria in chronic heart failure. Curr Drug Metab 2009;10:22-8.

2. Groschwitz KR, Hogan SP. Intestinal barrier function: molecular regulation and disease pathogenesis. J Allergy Clin Immunol 2009;124:3-20; quiz 21-2.

3. Rutten EP, Lenaerts K, Buurman WA, Wouters EF. Disturbed intestinal integrity in patients with COPD; effects of activities of daily living. Chest 2013.

4. Morris AJ, Howden CW, Robertson C, Duncan A, Torley H, Sturrock RD, Russell RI. Increased intestinal permeability in ankylosing spondylitis--primary lesion or drug effect? Gut 1991;32:1470-2.

5. Smecuol E, Sugai E, Niveloni S, Vazquez H, Pedreira S, Mazure R, Moreno ML, Label M, Maurino E, Fasano A, Meddings J, Bai JC. Permeability, zonulin production, and enteropathy in dermatitis herpetiformis. Clin Gastroenterol Hepatol 2005;3:335-41.

6. de Kort S, Keszthelyi D, Masclee AA. Leaky gut and diabetes mellitus: what is the link? Obes Rev 2011;12:449-58.

7. Compare D, Coccoli P, Rocco A, Nardone OM, De Maria S, Carteni M, Nardone G. Gut-liver axis: the impact of gut microbiota on non alcoholic fatty liver disease. Nutr Metab Cardiovasc Dis 2012;22:471-6.

8. Vivinus-Nebot M, Frin-Mathy G, Bzioueche H, Dainese R, Bernard G, Anty R, Filippi J, Saint-Paul MC, Tulic MK, Verhasselt V, Hebuterne X, Piche T. Functional bowel symptoms in quiescent inflammatory bowel diseases: role of epithelial barrier disruption and low-grade inflammation. Gut 2013.

9. Duerksen DR, Wilhelm-Boyles C, Parry DM. Intestinal permeability in long-term follow-up of patients with celiac disease on a gluten-free diet. Dig Dis Sci 2005;50:785-90.

10. Teixeira TF, Collado MC, Ferreira CL, Bressan J, Peluzio Mdo C. Potential mechanisms for the emerging link between obesity and increased intestinal permeability. Nutr Res 2012;32:637-47.

11. Furness JB, Clerc N. Responses of afferent neurons to the contents of the digestive tract, and their relation to endocrine and immune responses. Biological Basis for Mind Body Interactions 2000;122:159-172.

12. Pai VP, Horseman ND. Biphasic regulation of mammary epithelial resistance by serotonin through activation of multiple pathways. J Biol Chem 2008;283:3090110.

13. Drossman DA, Camilleri M, Mayer EA, Whitehead WE. AGA technical review on irritable bowel syndrome. Gastroenterology 2002;123:2108-31.

14. Spiller R, Aziz Q, Creed F, Emmanuel A, Houghton L, Hungin P, Jones R, Kumar D, Rubin G, Trudgill N, Whorwell P. Guidelines on the irritable bowel syndrome: mechanisms and practical management. Gut 2007;56:1770-98.

15. Mertz H, Naliboff B, Munakata J, Niazi N, Mayer EA. Altered rectal perception is a biological marker of patients with irritable bowel syndrome. Gastroenterology 1995; 109:40-52. 
16. Azpiroz F, Bouin M, Camilleri M, Mayer EA, Poitras P, Serra J, Spiller RC. Mechanisms of hypersensitivity in IBS and functional disorders. Neurogastroenterol Motil 2007; 19:62-88.

17. Gecse K, Roka R, Ferrier L, Leveque M, Eutamene H, Cartier C, Ait-Belgnaoui A, Rosztoczy A, Izbeki F, Fioramonti J, Wittmann T, Bueno L. Increased faecal serine protease activity in diarrhoeic IBS patients: a colonic lumenal factor impairing colonic permeability and sensitivity. Gut 2008;57:591-9.

18. Tooth D, Garsed K, Singh G, Marciani L, Lam C, Fordham I, Fields A, Banwait R, Lingaya M, Layfield R, Hastings M, Whorwell P, Spiller R. Characterisation of faecal protease activity in irritable bowel syndrome with diarrhoea: origin and effect of gut transit. Gut 2013.

19. Bueno L, Fioramonti J. Protease-activated receptor 2 and gut permeability: a review. Neurogastroenterol Motil 2008;20:580-7.

20. Cottrell GS, Amadesi S, Grady EF, Bunnett NW. Trypsin IV, a novel agonist of protease-activated receptors 2 and 4. J Biol Chem 2004;279:13532-9.

21. Annahazi A, Ferrier L, Bezirard V, Leveque M, Eutamene H, Ait-Belgnaoui A, Coeffier M, Ducrotte P, Roka R, Inczefi O, Gecse K, Rosztoczy A, Molnar T, Ringel-Kulka T, Ringel Y, Piche T, Theodorou V, Wittmann T, Bueno L. Luminal Cysteine-Proteases Degrade Colonic Tight Junction Structure and Are Responsible for Abdominal Pain in Constipation-Predominant IBS. Am J Gastroenterol 2013;108:1322-31.

22. Wilcz-Villega EM, McClean S, O'Sullivan MA. Mast cell tryptase reduces junctional adhesion molecule-A (JAM-A) expression in intestinal epithelial cells: implications for the mechanisms of barrier dysfunction in irritable bowel syndrome. Am J Gastroenterol 2013;108:1140-51.

23. Coeffier M, Gloro R, Boukhettala N, Aziz M, Lecleire S, Vandaele N, Antonietti M, Savoye G, Bole-Feysot C, Dechelotte P, Reimund JM, Ducrotte P. Increased proteasome-mediated degradation of occludin in irritable bowel syndrome. Am $\mathrm{J}$ Gastroenterol 2010;105:1181-8.

24. Bertiaux-Vandaele N, Youmba SB, Belmonte L, Lecleire S, Antonietti M, Gourcerol G, Leroi AM, Dechelotte P, Menard JF, Ducrotte P, Coeffier M. The expression and the cellular distribution of the tight junction proteins are altered in irritable bowel syndrome patients with differences according to the disease subtype. Am J Gastroenterol 2011;106:2165-73.

25. Wilder-Smith $\mathrm{CH}$. The balancing act: endogenous modulation of pain in functional gastrointestinal disorders. Gut 2011;60:1589-99.

26. Vergnolle N. Visceral afferents: what role in post-inflammatory pain? Auton Neurosci 2010;153:79-83.

27. Kilkens TO, Honig A, van Nieuwenhoven MA, Riedel WJ, Brummer RJ. Acute tryptophan depletion affects brain-gut responses in irritable bowel syndrome patients and controls. Gut 2004;53:1794-800.

28. Nishizawa S, Benkelfat C, Young SN, Leyton M, Mzengeza S, de Montigny C, Blier P, Diksic M. Differences between males and females in rates of serotonin synthesis in human brain. Proc Natl Acad Sci U S A 1997;94:5308-13.

29. Labus JS, Mayer EA, Jarcho J, Kilpatrick LA, Kilkens TO, Evers EA, Backes WH, Brummer RJ, van Nieuwenhoven MA. Acute tryptophan depletion alters the effective 
connectivity of emotional arousal circuitry during visceral stimuli in healthy women. Gut 2011;60:1196-203.

30. Jacobs BL, Fornal CA. Activity of serotonergic neurons in behaving animals. Neuropsychopharmacology 1999;21:9S-15S.

31. Dorn SD, Palsson OS, Thiwan SI, Kanazawa M, Clark WC, van Tilburg MA, Drossman DA, Scarlett Y, Levy RL, Ringel Y, Crowell MD, Olden KW, Whitehead WE. Increased colonic pain sensitivity in irritable bowel syndrome is the result of an increased tendency to report pain rather than increased neurosensory sensitivity. Gut 2007;56:1202-9.

32. Posserud I, Syrous A, Lindstrom L, Tack J, Abrahamsson H, Simren M. Altered rectal perception in irritable bowel syndrome is associated with symptom severity. Gastroenterology 2007; 133:1113-23.

33. Song GH, Venkatraman V, Ho KY, Chee MW, Yeoh KG, Wilder-Smith CH. Cortical effects of anticipation and endogenous modulation of visceral pain assessed by functional brain MRI in irritable bowel syndrome patients and healthy controls. Pain 2006; 126:79-90.

34. Levinthal DJ, Bielefeldt K. Differences in post-inflammatory hypersensitivity between splanchnic and pelvic afferents: mechanisms and implications for human disease. Gut 2009;58:1317-8.

35. Moussa L, Bezirard V, Salvador-Cartier C, Bacquie V, Houdeau E, Theodorou V. A new soy germ fermented ingredient displays estrogenic and protease inhibitor activities able to prevent irritable bowel syndrome-like symptoms in stressed female rats. Clin Nutr 2013;32:51-8.

36. Eswaran S, Goel A, Chey WD. What role does wheat play in the symptoms of irritable bowel syndrome? Gastroenterol Hepatol (N Y) 2013;9:85-91.

37. Vazquez-Roque MI, Camilleri M, Smyrk T, Murray JA, Marietta E, O'Neill J, Carlson P, Lamsam J, Janzow D, Eckert D, Burton D, Zinsmeister AR. A controlled trial of gluten-free diet in patients with irritable bowel syndrome-diarrhea: effects on bowel frequency and intestinal function. Gastroenterology 2013;144:903-911 e3.

38. Keszthelyi D, Masclee AA. Effects of proton pump inhibitor therapy in the distal gut: putting the pieces together. Dig Dis Sci 2012;57:2487-9.

39. Keszthelyi D, Masclee AA. Letter: lansoprazole consumption is more common in Japanese patients with collagenous colitis--authors' reply. Aliment Pharmacol Ther 2013;38:209-10. 
Discussion 
D. Keszthelyi

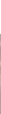


13

Appendices

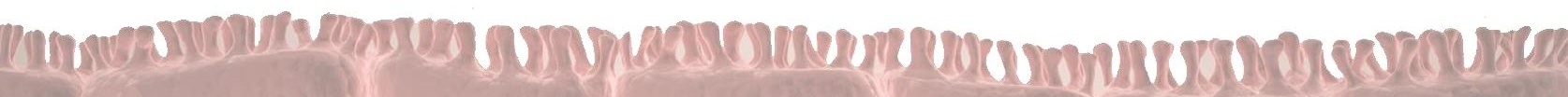




\subsection{SUMMARY}

This thesis investigated the (patho)physiology of intestinal barrier function. Previous studies indicate that an impairment of the intestinal barrier appears to play a key role in the development of several diseases. In this thesis, we investigated specific aspects of intestinal barrier function, with particular emphasis on two distinct gastrointestinal disorders, irritable bowel syndrome and microscopic colitis.

In Part I of this thesis, we examine the role of tryptophan metabolites in intestinal physiology, and in particular intestinal barrier function. Chapter 2 gives an overview of the current knowledge on this subject. Of the tryptophan metabolites, serotonin is the most well-described. It has a cardinal function in the orchestration of intestinal function. However, merely $1 \%$ of the ingested tryptophan is converted to serotonin. The overwhelming majority is subject to catabolism along the kynurenine pathway, which can give rise to a wide array of metabolites. Recent evidence suggests that these metabolites also play an important role in gastrointestinal function. Increasing attention has also been given to microbial degradation products of tryptophan breakdown, which is also discussed in this chapter.

In Chapter 3 of this thesis, we conducted a study using acute tryptophan depletion. This method is known to decrease serotonin synthesis in the central nervous system by decreasing the availability of the precursor tryptophan. We were interested whether a) such decrease in serotonin synthesis applying acute tryptophan depletion can also be achieved in the intestine and b) whether this can result in alterations in intestinal barrier function. Interestingly, we found that an acute decrease in the availability of the precursor of serotonin, tryptophan, did not result in a decrease in mucosal serotonin levels, suggesting that the intestine has a unique capacity to compensate for such changes. Hence, we observed no alterations in intestinal barrier function.

On the other hand, we provide evidence in this study for a competition between the two metabolic pathways, serotonin and kynurenine, for their mutual precursor, tryptophan. 
Following the acute stress response caused by the endoscopic examination applied in this study, we observed an increase in plasma serotonin levels, which was accompanied by a simultaneous decrease in kynurenic acid levels.

Such metabolic imbalance between the serotonin and kynurenine pathways has been suggested to play a role in disorders associated with serotonergic dysfunction. We were interested to find evidence whether such mechanism is also present in IBS. Therefore, in the study described in Chapter 4, we measured levels of serotonin and kynurenic acid in healthy controls and IBS. In IBS patients, we found decreased mucosal and increased systemic levels of both metabolites, suggesting an enhanced release of these substances from the intestine to the systemic compartment. This finding does not support the hypothetic role of a metabolic imbalance in the pathogenesis of IBS. On the other, it indicates that such a decrease in mucosal serotonin and kynurenic acid levels may result in disturbance of intestinal homeostasis, which can be of relevance to the pathogenesis of IBS.

Part II of this thesis focused on visceral hypersensitivity. Visceral hypersensitivity is a phenomenon frequently observed in patients with irritable bowel syndrome (IBS), and refers to augmented sensory responses to certain stimuli. Most often, rectal balloon distensions are used to assess visceral perception. An overview, including advantages and disadvantages of current techniques is given in Chapter 5 , with particular emphasis on novel techniques, such as brain imaging and neurophysiological recordings. Recent years have witnessed an increase on scientific knowledge on visceral perception through novel brain imaging modalities. However, a great number of the current techniques are largely influenced by less well-controlled or yet unknown psychological factors, which poses a challenge to investigating visceral nociception. Functional brain imaging studies are in need of well described and defined test paradigms and should be validated. In this chapter, we summarize recommendations in order to optimize future testing paradigms.

In Chapter 6, we discuss the different mechanisms involved in the pathogenesis of visceral hypersensitivity in IBS. Particular emphasis is given to the putative role of the 
impairment of the intestinal barrier, serotonin and microbial factors. Many authors consider low-grade inflammation as being an important pathophysiological factor in IBS. In chapter 6, we also provide evidence to demonstrate that this low-grade inflammation in fact can also be considered a consequence of increased barrier permeability and may therefore represent an epiphenomenon hereof.

With regards to a potential role of inflammation, postinflammatory visceral hyperalgesia is well-known phenomenon. However, the underlying mechanisms have not been fully elucidated. In Chapter 7, we provide evidence that patients with IBS and ulcerative colitis in remission exhibit increased transcription of TRPV 1, an important molecular integrator of nociceptive stimuli. Such upregulation of the TRPV 1 receptor can be in fact the result of previous inflammation. Increased TRPV 1 transcription and expression may result in increased peripheral discharge from visceral afferents, contributing to visceral hypersensitivity and chronic pain symptom generation, even in the absence of an apparent inflammatory reaction.

Part III of this thesis consists of Chapter 8. In this chapter, we aimed to integrate three different pathophysiological aspects of IBS: intestinal permeability, visceral perception and serotonin metabolism. Here, we demonstrated that increased serotonergic metabolism, following oral administration of the serotonin precursor 5hydroxytryptophan, resulted in an increase in visceral perception and induces an increase in intestinal mucosal barrier function in healthy individuals. We hypothesized that this reaction potentially represents a protective reflex to prevent penetration of noxious substances into the lamina propria. In IBS patients, an increased perceptive and metabolic response to 5-HTP accompanied a failure to reinforce intestinal integrity. This impairment of the intestinal barrier was seen in both normosensitive and hypersensitive IBS patients, whereas the nociceptive response was seen only in hypersensitive patients. Failure to reinforce barrier function in response to potentially noxious stimuli, signaled through serotonin, may therefore represent an important pathophysiological mechanism in IBS and, in particular, visceral hypersensitivity. 
Part IV of this thesis investigated the pathogenetic role of drugs in the development of IBS and microscopic colitis, a disease related to IBS in terms of symptomatology and pathogenesis. Both disorders are known to be associated with increased intestinal permeability. On the other hand, it has also been described that certain drugs, most importantly NSAIDs, have the potential to increase intestinal permeability and by virtue of acting so, can theoretically play a pathogenetic role in both IBS and microscopic colitis.

Chapter 9 describes a systematic review in which we investigate drug-induced microscopic colitis. Exposure to NSAIDs indeed appears to be significantly associated with the development of microscopic colitis. However, given the wide use of these drugs among the general population and the relative rarity of the condition, drug-induced $M C$ seems to be an uncommon complication, which most probably simply reflects an unfortunate idiosyncratic reaction to the particular drug. Nevertheless, a potential effect on the intestinal barrier may represent an important mechanism in such idiosyncratic reaction, which warrants further investigation.

In Chapter 10, we describe a case-control study, which demonstrates that the use of proton pump inhibitors (PPIs) is associated with microscopic colitis. These findings confirm the importance of considering PPI use as a possible etiological factor for microscopic colitis. Microscopic colitis may occur as an adverse reaction of PPIs and PPIs should be considered as a possible cause of diarrhea in patients exposed to these drugs.

In Chapter 11, we describe a similar case-control study conducted in patients with IBS. We found in study population of IBS patients a positive association with IBS and exposure to PPIs and NSAIDs, but also to SSRIs. The former two are associated with alterations of intestinal physiology, whereas the latter is often prescribed in IBS due to concomitant psychiatric comorbidity. While it is apparent that IBS patients utilize medication more often than controls, case-control studies generally do not allow interpretation with respect to a causal relation. Therefore, we considered this study as being hypothesis generating. Further research should include prospective evaluation of PPI users and NSAIDs users monitoring the development of IBS-symptoms in relation to drug exposure 
to ascertain whether this increased exposure to PPIs and NSAIDs should be considered as legitimate etiological factors in IBS.

Chapter 12 discusses the major findings of this thesis. Here, we also formulated some unifying hypotheses with regards to the pathogenetic role of the intestinal barrier function, the pathogenesis of IBS and the possible causes of visceral hypersensivity. Some future directions with regards to the potential role of drug exposure in microscopic colitis and IBS are also discussed. 


\subsection{SAMENVATTING}

In dit proefschrift werd de (patho)fysiologie van de intestinale barrièrefunctie onderzocht. Eerdere studies tonen aan dat een verstoring van de barrièrefunctie een essentiële rol kan spelen in de ontwikkeling van verschillende ziekten. In dit proefschrift onderzochten we specifieke aspecten van intestinale barrièrefunctie, met bijzondere nadruk op twee verschillende gastro-intestinale stoornissen, prikkelbare darmsyndroom (irritable bowel syndrome, IBS) en microscopische colitis.

In deel I van dit proefschrift onderzoeken we de rol van tryptofaan metabolieten in intestinale fysiologie, met name de intestinale barrièrefunctie. Hoofdstuk 2 geeft een overzicht van de huidige kennis over dit onderwerp. Serotonine is de meest bekende metaboliet van tryptofaan en heeft een belangrijke functie in de regulatie van darmfunctie. Echter, slechts $1 \%$ van de ingenomen tryptofaan wordt in serotonine omgezet. De overgrote meerderheid is onderworpen aan katabolisme langs de kynurenine route, waardoor een breed scala aan metabolieten kan worden aangemaakt. Recent onderzoek suggereert dat deze metabolieten ook een belangrijke rol spelen bij darmfunctie. Met name is er tegenwoordig veel interesse in de rol van microbiële afbraakproducten van tryptofaan, wat ook in dit hoofdstuk besproken wordt.

In Hoofdstuk 3 van dit proefschrift hebben beschrijven we een studie waarin gebruik is gemaakt van acute tryptofaan depletie. Deze methode is in het verleden vaak toegepast in de psychiatrie omdat het een daling van serotonine synthese veroorzaakt in het centrale zenuwstelsel door verlaging van de beschikbaarheid van de precursor van serotonine, tryptofaan. We waren geïnteresseerd of een dergelijke daling van de serotonine synthese na acute tryptofaan depletie ook in de darm bereikt kan woorden en of dit kan leiden tot veranderingen in de intestinale barrièrefunctie. Interessant genoeg vonden we dat een afname van de beschikbaarheid van de precursor van serotonine, tryptofaan, niet tot een afname van het mucosale serotonine gehalte heeft geleid. Dit suggereert dat de darm een uniek vermogen heeft om te compenseren voor acute veranderingen in het aanbod aan de precursor. Waarschijnlijk is dit ook de oorzaak voor 
het feit dat we ook geen veranderingen in intestinale barrièrefunctie hebben waargenomen.

Anderzijds tonen we aan in deze studie dat er een competitie bestaat tussen de twee routes van tryptofaan metabolisme, serotonine en kynurenine. Na de acute stressreactie, veroorzaakt door het endoscopisch onderzoek toegepast in deze studie, zagen wij een toename in plasma serotonine gehalte, die gepaard ging met een gelijktijdige vermindering in de plasmaconcentraties van kynureninezuur, een van de metabolieten van de kynurenine route.

Eerder onderzoek heeft gesuggereerd dat een metabool dysbalans tussen de serotonine en kynurenine routes een rol kan spelen bij aandoeningen geassocieerd met serotonerge dysfunctie. Verhoogde activiteit langs de kynurenine metabole route zou theoretisch kunnen leiden tot een tekort aan serotonine, en andersom. We waren geïnteresseerd of er aanwijzingen zijn voor de aanwezigheid van een dergelijk mechanisme bij prikkelbare darmsydnroom. In Hoofdstuk 4 beschrijven we een studie, waarbij we de concentraties van serotonine en kynureninezuur hebben gemeten in gezonde controles en patiënten met IBS. We vonden in patiënten met IBS verminderde mucosale en verhoogde plasmaconcentraties van zowel serotonine als kynureninezuur. Dit suggereert een verhoogde afgifte van deze stoffen uit de darm naar het systemische compartiment. Hierdoor lijkt een metabole competitie tussen de twee routes minder waarschijnlijk. Anderzijds betekent dit dat een daling van mucosale serotonine en kynureninezuur gehalte kan leiden tot een verstoring van intestinale homeostase, die relevant kan zijn voor de pathogenese van IBS.

Deel II van dit proefschrift richt zich op viscerale overgevoeligheid, oftewel hypersensitiviteit. Viscerale overgevoeligheid is een fenomeen die vaak gezien wordt bij patiënten met prikkelbare darmsyndroom. Viscerale overgevoeligheid betekent een versterkt pijnrespons op bepaalde prikkels. In de klinische praktijk wordt dit getest middels de zogeheten rectale barostat methode, waarbij een ballon rectaal wordt ingebracht en tot bepaalde drukken opgeblazen. Hierbij wordt het gevoel van de patiënt geregistreerd. 
In Hoofdstuk 5 geven we een overzicht van de huidige technieken om viscerale overgevoeligheid te onderzoeken. De afgelopen jaren zagen we een enorme toename van wetenschappelijke kennis op gebied van viscerale perceptie dankzij de introductie van nieuwe imaging technologieën, zoals de fMRI. Ondanks de initiële enthousiasme zijn er echter nog een aantal uitdagingen op dit gebied. Met name moeten deze nieuwe methoden nog goed gevalideerd worden, vooral omdat de uitkomstparameters zich sterk laten beïnvloeden door psychologische factoren. In dit hoofdstuk geven hoe de methodologie rondom viscerale hypersensitiviteit verder geoptimaliseerd kan worden.

In Hoofdstuk 6 bespreken we de verschillende mechanismen die betrokken zijn bij de pathogenese van viscerale hypersensitiviteit bij IBS. Een bijzondere aandacht wordt besteed aan de mogelijke rol van de een verstoring van de intestinale barrière, serotonine en microbiële factoren. Velen beschouwen laaggradige ontsteking in de mucosa als een belangrijke pathofysiologische factor bij IBS. In hoofdstuk 6 bespreken we dat laaggradige ontsteking in feite ook kan worden beschouwd als een gevolg van de toegenomen permeabiliteit. Hierdoor blijkt de barrièrefunctie een centrale rol te spelen bij de pathogenese van IBS.

Mucosale ontsteking kan ook leiden tot verhoogde sensitisatie van pijnreceptoren in de mucosa, waardoor er meer pijnsignalen gegenereerd kunnen worden, resulterend in een toename van subjectieve pijnsensaties. Dit fenomeen is bekend als post-inflammatoire viscerale hyperalgesie. Echter, de onderliggende mechanismen zijn nog niet volledig opgehelderd. In Hoofdstuk 7 laten we zien dat patiënten met IBS en colitis ulcerosa in remissie (dus patiënten zonder evidente tekenen van een actieve mucosale ontstekingsreactie) een verhoogde mucosale transcriptie van het molecuul TRPV1 vertonen. TRPV 1 is een belangrijke moleculaire integrator van perifere nociceptieve stimuli, activatie hiervan lijdt tot het genereren van een pijnsignaal. Een dergelijke upregulatie van TRPV1 kan in feite het resultaat zijn van een eerdere ontsteking. Persisterende toename van TRPV 1 transcriptie en expressie kan leiden tot verhoogde activiteit van perifere viscerale afferenten, ook al is er geen sprake meer van een actieve 
mucosale ontsteking. Dit zou ook het chronische karakter van de pijnklachten in deze groep patiënten kunnen verklaren.

Deel III van dit proefschrift bestaat uit Hoofdstuk 8. In dit hoofdstuk hebben we ons gericht op drie verschillende pathofysiologische aspecten van IBS: darmpermeabiliteit, viscerale perceptie en serotonine metabolisme. Hier toonden we aan dat verhoogde serotonerge metabolisme na orale toediening van de serotonine precursor 5hydroxytryptofaan (5-HTP), resulteerde in een verhoging van viscerale perceptie en induceert een toename van intestinale mucosale barrièrefunctie bij gezonde individuen. Onze hypothese was dat deze reactie geschiedt in het kader van een protectieve reflex om het binnendringen van schadelijke stoffen in de lamina propria te voorkomen. Bij IBSpatiënten zagen we een verhoogde pijnreactie en metabole respons op 5-HTP, waarbij er geen versterking van de intestinale integriteit gezien werd. Het onvermogen om de intestinale barrière te versterken, als reactie op de luminale aanwezigheid van een potentieel schadelijke stof kan een belangrijke pathofysiologische mechanisme in IBS vertegenwoordigen.

Deel IV van het proefschrift onderzochten de pathogenetische rol van geneesmiddelen in de ontwikkeling van IBS en microscopische colitis, een aandoening die gerelateerd is aan IBS wat betreft symptomatologie en pathogenese. Beide aandoeningen zijn geassocieerd met verhoogde intestinale permeabiliteit. Anderzijds is het ook bekend dat bepaalde medicijnen, vooral NSAIDs, de intestinale permeabiliteit kunnen verhogen en hierdoor theoretisch een pathogenetische rol kunnen spelen bij IBS en microscopische colitis.

Hoofdstuk 9 beschrijft een systematische review waarin we kijken naar het gebruik van geneesmiddelen als mogelijke oorzaak voor microscopische colitis. Blootstelling aan NSAIDs lijkt inderdaad significant geassocieerd met de ontwikkeling van microscopische colitis. Echter, gezien het uitgebreide gebruik van deze geneesmiddelen onder de algemene bevolking en de relatieve zeldzaamheid van de aandoening, lijkt geneesmiddelgeïnduceerde microscopische colitis een ongewone complicatie, die waarschijnlijk berust op een idiosyncratische reactie op het specifieke geneesmiddel. Niettemin kan een 
potentieel effect op de darmwand een belangrijke rol spelen in een dergelijke idiosyncratische reactie, die verder onderzoek rechtvaardigt.

In Hoofdstuk 10 beschrijven we een case-control studie, waaruit blijkt dat het gebruik van protonpompremmers (PPI's) geassocieerd is met microscopische colitis. Indien er een patiënt met microscopische colitis gezien wordt, moet dus blootstelling aan PPIs overwogen worden als mogelijke uitlokkende factor en een poging gedaan worden om deze medicatie te staken.

In Hoofdstuk 11 beschrijven we een soortgelijke case-control studie uitgevoerd bij patiënten met IBS. We vonden in onze populatie van IBS-patiënten een positieve associatie met IBS en blootstelling aan de geneesmiddelen PPI en NSAIDs, maar ook bepaalde antidepressiva (SSRIs). De eerste twee groepen geneesmiddelen worden geassocieerd met veranderingen van intestinale fysiologie, terwijl de laatste vaak wordt voorgeschreven bij IBS wegens gelijktijdige psychiatrische comorbiditeit. Hoewel het duidelijk is dat patiënten met IBS vaker geneesmiddelen gebruiken dan controles, zijn case-control studies niet geschikt om een causaal verband te bevestigen tussen een geneesmiddel en een bepaalde aandoening. Daarom beschouwen we deze studie vooral als hypothese genererend. Verder prospectief onderzoek van IBS patienten moet aantonen of het gebruik van PPIs of NSAIDs de symptomen van IBS kan uitlokken en of een verhoogde blootstelling aan protonpompremmers en NSAIDs kan worden beschouwd als legitieme etiologische factoren in IBS.

Hoofdstuk 12 bespreekt de belangrijkste bevindingen van dit proefschrift. Hier hebben we ook enkele overkoepelende hypotheses geformuleerd met betrekking tot de pathogenetische rol van de intestinale barrièrefunctie, de pathogenese van IBS en de mogelijke oorzaken van viscerale overgevoeligheid. Mogelijke richtingen voor vervolgonderzoek met betrekking tot de potentiele rol van blootstelling aan bepaalde geneesmiddel in microscopische colitis en IBS worden ook besproken. 


\subsection{VALORISATION ADDENDUM}

The economic and cultural pressure on healthcare caused by an ageing population and higher expectations of the quality and accessibility of care can only increase. The healthcare sector in particular has to deal with the rising demand for services in addition to limited budgetary conditions. Innovative approaches in healthcare are needed to ensure providing universal high quality healthcare remains sustainable.

This is the most important incentive to perform research in medicine. Even basic research has the potential to alter clinical practice, resulting in greater efficacy, better outcomes and fewer side effects, even though the translation of such findings to everyday life may take longer than policy makers and research funders might wish for.

In this thesis, we explored a number of scientific questions in an attempt to provide answers for clinical problems derived from everyday practice. We can summarize these as follows.

1. In this thesis, we have examined the potential mechanisms underlying irritable bowel syndrome (IBS). IBS is characterized by chronic recurrent abdominal pain and disturbed defecation in absence of structural or biochemical abnormalities that could explain the symptoms. IBS affects $15-20 \%$ of women and $5-20 \%$ of men in the Netherlands ${ }^{1}-$ these $^{2}$ figures are comparable to those from other Westerns countries. This means that there are approximately 120 thousand patients in the Netherlands who suffer from IBS. Currently, a very limited number of effective therapeutic entities are available for IBS. Due to the lack of effective therapies and the relatively high rates of prevalence, direct healthcare related costs as well as indirect costs related to work-absenteeism and presenteeism are considerable. IBS patients often utilize multiple drugs over longer periods of time, resulting in high costs for medicine use. Furthermore, about one-third of the patients also suffer from psychiatric comorbidity often needing pharmacological or psychological interventions. 
Overall, health care seeking is greater in IBS patients than in non-IBS patients. ${ }^{2}$ Nevertheless, only approximately $30 \%$ of IBS patients consult a physician. ${ }^{3}$ Of these patients, $80 \%$ is seen only by general practitioners, $20 \%$ also by secondary care specialists. ${ }^{4}$ Annual direct costs associated with healthcare for IBS patients in the Netherlands are estimated to be $€ 1648$ in primary care and $€ 2003$ for patients seen in secondary care (personal communication, C. Flik and W. Laan, Julius Center, Utrecht). Physician costs account for $60 \%$ of the costs, medication for $20 \%$ and hospital stay for $20 \% .^{5}$ On average, 1.6 contacts each year per patient are made by GPs and 2.14 contacts for gastroenterologist every year. ${ }^{5}$ Only a small minority of $2.5 \%$ will be admitted for an average stay of 5 days. $^{6}$

Besides direct healthcare costs, indirect costs from IBS might have an even higher economic impact. With regards to societal costs, IBS is associated with average 8 days of absenteeism annually. ${ }^{7}$ In addition, the disease burden causes $21 \%$ reduction in work productivity, equivalent to working less than 4 days in a 5 -day workweek. ${ }^{8}$ Considering an average of 228 working days per year, absenteeism and presenteeism accounts for 54 working day losses (equals $€ 7344$ annually assuming an 8-hour work day and an average hourly wage of $€ 17$ ). The NHG guideline estimates up to 48 days of annual absenteeism based on European data that does not account for presenteeism. ${ }^{1}$ Considering 120 thousand IBS patients and 30\% healthcare utilization, adding up direct and indirect costs of IBS results in an estimate of over $€ 940$ million per year.

These figures illustrate that further research in IBS is not only necessary from a patients' point of view by providing therapies aimed to decrease disease burden, but also has the potential to result in an economic and societal benefit. We therefore consider the findings in this thesis as a step forward in developing efficacious therapeutic entities for IBS.

2. One of these potential novel therapeutic entities is related to restoration of the impaired barrier function. This thesis has focused on the role of the intestinal barrier in the development of gastrointestinal disorders, such as IBS. However, as explained in this thesis, impaired barrier function has been associated with a wide array of diseases, such 
as diabetes and metabolic syndrome. It is generally appreciated that these diseases represent an enormous burden on the healthcare budget and that every effort to decrease the morbidity caused by these diseases will be applauded by patients and policy makers alike.

One might consider the reinforcement of the intestinal barrier function as an entity which may not carry a high therapeutic potential. However, very recently, the results of the first trial using a novel class of agents targeting tight-junction regulation was presented at the Digestive Disease Week in $2014 .{ }^{9}$ Investigators treated celiac disease patients with larazotide acetate, a first-in-class oral peptide, which prevents tight junction opening and reduces gluten uptake, inhibiting gluten- and cytokine-induced intestinal permeability and inflammation in vivo. The treatment resulted in a significant reduction of symptoms.

We therefore anticipate that future research will further substantiate the evidence for targeting tight junctions as a legitimate therapeutic entity in the treatment of a wide spectrum of disorders.

3. A substantial part of this thesis concerns the potential etiological role for drug exposure in two distinct gastrointestinal disorders, IBS and microscopic colitis (MC). Both patient groups are prone to over-utilization of drugs; IBS patients are characterized by healthcare-seeking behavior and MC patients are generally elderly. Although the results provided in this thesis do not allow the establishment of a cause-effect relationship, it is of particular importance to critically review the necessity of prescribing certain medications in these particular patients.

Adverse drug events are a burden on healthcare systems because of the resources needed to diagnose and treat the symptoms and diseases caused by them. From the perspective of society, these costs are unnecessary, regardless of the point of origin. A recent study performed at our hospital showed that over $40 \%$ of direct healthcare costs of hospital admissions were due to adverse events related to medication. ${ }^{10}$ Another recent population-based study from Sweden demonstrates that adverse drug events 
resulted in direct healthcare cost of USD 445 per patient in period of three-months, with $44 \%$ of these direct costs occurring outside inpatient care settings. In addition, a patient with adverse drug events resulted in an additional USD 6235 of total societal cost in the period examined. ${ }^{11}$ Although these studies also include adverse events related to medication other than NSAIDs and PPIs - the drugs found to be positively associated with IBS and MC, they are indicative of the budgetary impact of adverse drug reactions on healthcare.

\section{REFERENCES}

1. IBS guidelines of the Dutch College of General Practitioners (NHG), Huisarts Wet 2012;55:204-9.

2. Spiller R, Aziz Q, Creed F et al. Clinical Services Committee of the British Society of Gastroenterology. Guidelines on the irritable bowel syndrome: mechanisms and practical management. Gut 2007;56:1770-98.

3. Drossman DA, Camilleri M, Mayer EA, Whitehead WE. AGA technical review on irritable bowel syndrome. Gastroenterol 2002;123:2 108-31.

4. Thomson WG, Heaton KW, Smyth GT, Smyth C. Irritable bowel syndrome in general practice: prevalence, characteristics, and referral. Gut 2000; 46:78-82.

5. Delvaux. Functional bowel disorders and irritable bowel syndrome in Europe. Aliment Pharm Therap 2003; 18 Suppl 3:75-9.

6. Wells NE, Hahn BA, Whorwell PJ. Clinical economics review: irritable bowel syndrome. Aliment Pharm Therap 1997;11:1019-30.

7. Cash B, Sullivan S, Barghout V. Total costs of IBS: employer and managed care perspective. Am J Manag Care 2005; 1 (1 Suppl):S7-16.

8. Leong SA, Barghout V, Birnbaum HG, Thibeault CE, Ben-Hamadi R, Frech F, Ofman JJ. The economic consequences of irritable bowel syndrome: a US employer perspective. Arch Intern Med 2003;28;163:929-35.

9. Wang C, Rasmussen H, Perrow W, Kelly C, Leffler D, Green O, Fedorak RN, DiMarino AJ, Bercik P, Murray JA, Bachir NM. Larazotide Acetate, a First In-Class, Novel Tight Junction Regulator, Meets Primary Endpoint and Significantly Reduces Signs and Symptoms of Celiac Disease in Patients on a Gluten-Free Diet: Results of a Multicenter, Randomized, Placebo Controlled Trial. Gastroenterol 2014;146:Suppl 1:S-159.

10. Magdelijns FJ, Stassen PM, Stehouwer CD, Pijpers E. Direct health care costs of hospital admissions due to adverse events in the Netherlands. Eur J Public Health. 2014 Apr 2. [Epub ahead of print]

11. Gyllensten H, Hakkarainen KM, Hagg S, Carlsten A, Petzold M, Rehnberg C, Jönsson AK. Economic impact of adverse drug events--a retrospective population-based cohort study of 4970 adults. PLoS One 2014;9:e92061. 


\subsection{DANKWOORD}

Mijn sollicitatiegesprek kan ik me nog heel goed herinneren. Ik zat aan tafel met Prof. Masclee en Prof. Dejong. Het was ergens eind maart 2008 en ik moest toen nog mijn artsenbul halen. Prof. Dejong vroeg of ik wel zeker wist óf ik het ging halen. En van Prof. Masclee moest ik toch aan mijn algemeen beschaafd Nederlands werken, want er zat nog te veel Limburgs tussen. Dat eerste is me in ieder geval inmiddels gelukt. Vervolgens moest ik me voorstellen bij Prof. Buurman. Toen kreeg ik het verhaal voor het eerst te horen dat hij in de jaren "60 over de grens moest tussen Hongarije en toenmalig Joegoslavië en dat hij van de douane alle spullen uit de tassen moest halen van tandenborstel tot vuil ondergoed.

In een zekere zin wist toen niemand hoe het zal aflopen met de 'gekke Hongaar,' om maar eens de woorden van Prof. Dejong te gebruiken. Of naar collega's Karen Koning en Jeroen Maljaars: het VOB'ertje (voormalig Oost-blokker). Zoals de titel van het nieuwe boek van Hillary Clinton, hier voor me op tafel, aangeeft, gaat het vaak in het leven om het maken van 'cruciale keuzes.' Zes jaar na dato kan ik met volle overtuiging zeggen dat de 'gekke Hongaar' en het MUMC+ toch wel een goede match zijn geworden. Bovenal heb ik in tijdens met promotie een toptijd gehad en genoten van de gezelschap van collega's en vrienden waar ik mee samen mocht werken. Hiervoor ben ik velen dank verschuldigd.

Allereerst mijn dank aan mijn promotor Prof. Ad Masclee. Dank voor het vertrouwen, de onvoorwaardelijke ondersteuning. Ik bewonder uw gedrevenheid en gevoel voor diplomatiek. Om toch maar weer een anekdote te noemen vond ik ons tripje naar mijn geboortestad Pécs samen met Daisy en Samefko één van de hoogtepunten van mijn promotietijd. Wij stonden op het vliegveld van Boedapest, u vloog met Daisy terug naar Nederland, Samefko en ik gingen nog een paar dagen Boedapest onveilig maken. Uw 'vaderlijke' woorden van afscheid waren toen: 'Daniel, denk aan twee dingen: kwaliteit en veiligheid. Doen we ook in het ziekenhuis.' 
Ik ben heel enthousiast over de onderzoeksplannen die we samen hebben gemaakt. Ik hoop dat onze vakgroep in Maastricht een leidende positie gaat innemen in de wereldtop van neurogastroenterologie.

Freddy. Jouw wegwijs in de wereld van onderzoek, en met name regelgeving, logistiek en organisatie was vooral in het begin van mijn promotie onmisbaar. We hebben frustraties samen gedeeld maar uiteindelijk is het ons gelukt om alles op de rails te zetten. En het resultaat mag er ook zijn. Bedankt voor het feit dat je me de vrijheid gaf om mijn eigen wegen te bewandelen. Bedankt voor de gezelligheid tijdens tripjes naar congressen en andere uitstapjes. Ik zal onze shoppingtour (lees: congresbezoek) naar Boston nooit vergeten.

Prof. Buurman. Wat heb ik veel van u geleerd. Ik sta vaak met verbazing te kijken naar de enorm brede kennis en ervaring die u heeft. Ik denk graag terug aan de vele uren die we hebben doorgebracht om mijn manuscripten te verbeteren (de eerste versies waren veelal waardeloos) met ertussen nog vele verhalen rondom de thema's Oostblok, paarden en politiek binnen en buiten het MUMC. Ik hoop dat ik in de toekomst nog vaak beroep kan doen op uw expertise.

Daisy. Ook al was je officieel niet mijn co-promotor, heb ik ook ontzettend veel aan jou te danken. Mede door het feit dat jouw kamer naast de mijne was kon ik altijd even bij je binnenlopen als ik wat hulp nodig had, en je was altijd behulpzaam. Bedankt ook voor de gezellige avonden met Karel erbij! Ik hoop dat we in de toekomst nog hele leuke dingen samen kunnen bereiken.

De leden van de beoordelingscommissie wil ik graag bedanken voor de tijd die ze hebben genomen voor het doorlezen en beoordelen van mijn proefschrift. Naast voorzitter Prof. Schols, bestond de commissie uit Prof. Dejong, Prof. De Jonge, Prof. Steinbusch en Prof. Aziz. 
Mijn paranimfen. Allereerst Samefko; Batman and Robin, Pinky and the Brain, Jut en Juul (Annemiek Thijssen), the 'Gl twins' (Guy Vijgen) - zo werden wij wel eens aangeduid. Als iemand één van ons zag lopen, kon je de andere binnen $10 \mathrm{~m}$ afstand ook verwachten. Dankzij jou heb ik hier mijn draai kunnen vinden in Maastricht. Ik ben je daar zeer dankbaar voor. Mark, jij maakt ons team van de drie musketiers compleet. Datgene wat we samen hebben meegemaakt in die paar jaar tijd verdient bijna ook een apart hoofdstuk, zo niet een heel boek.

Dank aan mijn eerste kamergenote Karen. Wat fijn dat je me in het begin onder je vleugels hebt genomen. Dank aan de overige collega onderzoekers van de MDL: Jeroen, Jeoffrey, Carolina, Henrike, Steven, Andrea, Maartje, Tessa, Martine, Suzanne, Annemiek, Eveline, Kirsten, Esther, Bouke, Mariëlle, Elhaseen, Harm-Jan, Montserrat, Zlatan, Chantal, Paul, Renske, Corinne, Steven, Bas, Kirsten, Fedde, Fabienne, Tim, Ellen. Dank aan de studenten die ook voor een groot gedeelte hebben bijgedragen aan het onderzoek: Ronald, Timo, Tijs, Thomas, Jordi, Gwen D, Gwen M, Anna, Lieneke, Nathalie. Dank aan de collega's van de afdeling chirurgie, in het bijzonder Kim, Kaatje, Joep, Hans, Babs, Tim, Mo, Marlou, Nina, Froukje en Charlotte. En collega's van het lab interne geneeskunde (Casper, Jean, Olaf, Dennis, Nordin) en pulmonologie (Alex en Koen).

Dank aan alle proefpersonen, die mee hebben gedaan aan dit onderzoek. Zonder hen was dit allemaal onmogelijk geweest.

Dank aan de afdeling endoscopie, Ton en alle endoscopie-verpleegkundigen en dank aan alle MDL-artsen en arts-assistenten, die mij zo vaak hebben geholpen op de scopiekamer. Dank aan de medewerkers van de functiekamer, Pierre, Helene, Ria, Andrea en Nicole. Dank aan het secretariaat MDL, Mietsie en Elly. Jullie zijn top!

Dank aan TIFN, die mijn promotie mogelijk heeft gemaakt en onze projectleider Jan Dekker. Ook dank aan de andere TIFN A 1001 collega's uit Wageningen en Groningen voor de waardevolle bijeenkomsten en discussies. Dank eveneens aan de onderzoeksschool NUTRIM, en in het bijzonder Sef Janssen. Dank aan alle co-auteurs voor de constructieve 
samenwerking, in het bijzonder Carsten Leue, Patrick Lindsey, John Penders, Sita Jansen, Joanna Kruimel en Marieke Pierik.

Thanks to Michel Neunlist of the University of Nantes, for allowing me to work in your lab. I enjoyed my stay very much and hope we can continue working together in the future!

My special thanks to Prof. Zsuzsanna Helyes of the Department of Pharmacology, University of Pécs. I have had the honour of doing research as a student under your supervision. I admire your energetic and ever-enthusiastic character. I hope our collaborative efforts will result in many more outstanding publications. I would also like to express my gratitude to Prof. Szolcsányi, Prof. Pintér and other co-workers of the department. I hope this thesis may carry on the tradition of the outstanding Hungarian scientists in the field of pain research.

Khaya, bedankt voor de nachtelijke uren die je hebt gespendeerd aan de layout van dit proefschrift.

Dank aan al mijn vrienden. In het bijzonder Sander, Faro, Bart, Stef en Dalilah.

Bijzonder dank aan Gerda. Wie had zich kunnen voorstellen dat ik hier zou komen te staan toen ik bij Jo en Gerda in huis kwam als 16-jarig jongetje. Dankzij jullie is dit allemaal mogelijk geworden. De warmte die ik bij jullie in huis voelde heeft Stein voor me een tweede thuis gemaakt zoals voor nog velen. Ik voel me geprezen dat ik me ook een van jullie kinderen mag noemen. Dank aan mijn twee grote broers Hélder en Ruben, en hun partners Nikki en Nina. Dank aan Marion en Frank, Roger en Bianca, Stef en Niek (met name ook voor het meedoen aan mijn onderzoek) en de rest van mijn tweede familie in Stein.

I owe much gratitude to my parents and in particular to my mother. Mother, I am grateful for all you have done for me. All I have achieved in life would not have been possible without you. You have also made 'hard choices' in life. Choices, which proved to be the 
right ones and from which I still benefit every day of my life. I am glad to see that your grandson makes you so happy.

I am grateful to my grandparents, who have always surrounded me with so much love and affection, and especially my grandfather, who would have been the happiest man on earth if he could have lived to this day.

Rilana, jij bent de liefde van mijn leven. Ik ben heel gelukkig met jou en met onze prachtige zoon Valentijn. 


\subsection{LIST OF PUBLICATIONS}

Keszthelyi D, van Avesaat M, Knol D, Troost FJ, Ludidi S, Sleijpen R, Gribnau M, Foltz M, Masclee AAM. Effect of meal ingestion on pharmacokinetics of acetaminophen administered in a liquid formulation: revisiting concepts of gastric emptying. In preparation

Keszthelyi D, Troost FJ, Jonkers DM, van Eijk HM, Dekker J, Buurman WA, Masclee AAM. Visceral hypersensitivity in irritable bowel syndrome: evidence for involvement of serotonin metabolism. Submitted

Mujagic Z, Keszthelyi D, Aziz Q, Reinisch W, Quetglas EG, Segerdahl M, De Leonardis F, Masclee AAM. An extensive review on instruments to assess chronic abdominal pain in IBS and IBD: reflections on questions and quality. Submitted

Verhaegh B, Jonkers DM, Driessen A, Zegers M, Keszthelyi D, Masclee AAM, Pierik M. Incidence of Microscopic Colitis in the Netherlands. A Nationwide Population-Based Study from 2000-2012. Submitted

Smeets F, Conchillo JM, Keszthelyi D, Bouvy N, Masclee AAM. Preoperative Esophagogastric Junction (EGJ) Distensibility predicts treatment outcome after endoluminal fundoplication in GERD patients. Submitted

Keszthelyi D, Masclee AAM. Letter: Capsaicin stimulation in irritable bowel syndrome: towards understanding visceral perception and pain symptom generation. American Journal of Gastroenterology 2014 Aug; 109(8):1286

Keszthelyi D, Troost FJ, Jonkers DM, van Eijk H, Lindsey P, Dekker J, Buurman WA, Masclee AAM. Serotonergic reinforcement of intestinal barrier function is impaired in irritable bowel syndrome. Alimentary Pharmacology and Therapeutics 2014 Aug;40(4):392-402. 
Mujagic Z, Ludidi S, Keszthelyi D, Hesselink M, Kruimel JW, Lenaerts K, Hanssen NM, Conchillo J, Jonkers DM, Masclee AAM. Small intestinal permeability is increased in diarrhoea predominant IBS, while alterations in gastroduodenal permeability in all IBS subtypes are largely attributable to confounders. Alimentary Pharmacology and Therapeutics 2014 Aug;40(3):288-97.

Ludidi S, Mujagic Z, Jonkers DM, Keszthelyi D, Hesselink M, Kruimel JW, Conchillo JM, Masclee AAM. Markers for visceral hypersensitivity in patients with irritable bowel syndrome. Neurogastroenterology and Motility 2014 Aug;26(8):1 104-11.

Keszthelyi D, Jonkers DM, Hamer HM, Masclee AAM. Letter: The role of sub-clinical inflammation and TRPV 1 in the development of IBS-like symptoms in ulcerative colitis in remission. Alimentary Pharmacology and Therapeutics 2013 Sep;38(5):560-1

Keszthelyi D, Masclee AAM. Letter: Lansoprazole consumption is more common in Japanese patients with collagenous colitis - authors' reply. Alimentary Pharmacology and Therapeutics 2013 Jul;38(2):209-10

Keszthelyi D, Troost FJ, Jonkers D, Kruimel JW, Leue C, Masclee AAM. Decreased levels of kynurenic acid in the intestinal mucosa of IBS patients: relation to serotonin and psychological state Journal of Psychosomatic Research 2013 Jun;74(6):50 1-4

Keszthelyi D, Troost FJ, Jonkers D, Helyes Z, Hamer HM, Ludidi S, Vanhoutvin S, Venema K, Dekker J, Szolcsanyi J, Masclee AAM. Alterations in mucosal neuropeptides in irritable bowel syndrome: a role in pain symptom generation? European Journal of Pain 2013 Oct; 17(9): 1299-306

Keszthelyi D, Penders J, Masclee AAM, Pierik M. Is microscopic colitis a drug-induced disease? Journal of Clinical Gastroenterology 2012 Nov-Dec;46(10):811-22. 
Keszthelyi D, Dackus G, Masclee GM, Kruimel JW, Masclee AAM. Increased proton pump inhibitor and NSAID exposure in irritable bowel syndrome: results from a case-control study. BMC Gastroenterology 2012 Sep 5;12(1):121.

Keszthelyi D, Knol D, Troost FJ, van Avesaat M, Foltz M, Masclee AA. Time of ingestion relative to meal intake determines gastrointestinal responses to a plant sterol containing yoghurt drink. European Journal of Nutrition 2013 Jun;52(4):1417-20.

Keszthelyi D, Masclee AAM. Effects of proton pump inhibitor therapy in the distal gut: putting the pieces together. Digestive Diseases and Sciences 2012 Oct;57(10):2487-9.

Keszthelyi D, Troost FJ, Masclee AAM. Methods to assess visceral hypersensitivity in irritable bowel syndrome. American Journal of Physiology-Gastrointestinal Liver Physiology 2012 Jul;303(2):G141-54.

Peeters M, Troost FJ, van Grinsven B, Horemans F, Alenus J, Murib MS, Keszthelyi D, Ethirajan A, Thoelen R, Cleij TJ, Wagner P. MIP-based biomimetic sensor for the electronic detection of serotonin in human blood plasma. Sensors and Actuators B, Chemical 2012 Aug-Sept(171-172):602-610.

Ludidi S, Conchillo J, Keszthelyi D, van Avesaat M, Kruimel JW, Jonkers DM, Masclee AAM. Rectal hypersensitivity as hallmark for irritable bowel syndrome: defining the optimal cut-off. Neurogastroenterology and Motility 2012 Aug;24(8):729-e346.

Keszthelyi D, Troost FJ, Simrén M, Ludidi S, Kruimel JW, Conchillo J, Masclee AAM. Revisiting concepts of visceral nociception in irritable bowel syndrome. European Journal of Pain 2012 Nov; 16(10): 1444-54.

Keszthelyi D, Troost FJ, Jonkers DM, van Donkelaar EL, Dekker J, Buurman WA, Masclee AAM. Does acute tryptophan depletion affect peripheral serotonin metabolism in the intestine? American Journal of Clinical Nutrition 2012 Mar;95(3):603-8. 
van Avesaat M, Keszthelyi D, Masclee AAM. The gut: key to treating obesity in adolescents? Journal of Pediatric Gastroenterology and Nutrition 2011 Dec;53(S2):S2526.

Ludidi S, Conchillo JM, Keszthelyi D, Koning CJ, Vanhoutvin SA, Lindsey PJ, Leufkens AM, Kruimel JW, Jonkers D, Masclee AA. Does meal ingestion enhance sensitivity of visceroperception assessment in irritable bowel syndrome? Neurogastroenterology and Motility 2012 Jan;24(1):47-53, e3.

Wen LS, Greysen R, Keszthelyi D, Bracero J, de Roos PDG. Letter: The Need for Social Accountability in Health Professional Education: A Call to Service from Young Doctors. Lancet 2011 Dec 3;378(9807):e12-3.

de Kort S, Keszthelyi D, Masclee AAM. Leaky gut and diabetes mellitus: What is the link? Obesity Reviews 2011 Jun;12(6):449-58.

Keszthelyi D, Jansen SV, Schouten GA, de Kort S, Scholtes B, Engels LG, Masclee AAM. Proton pump inhibitor use is associated with an increased risk for microscopic colitis: a case-control study. Alimentary Pharmacology and Therapeutics 2010 Nov;32(9):1124-8.

Maljaars PW, Keszthelyi D, Masclee AAM. An ileal break-through? American Journal of Clinical Nutrition 2010 Sep;92(3):467-8.

Varecza Z, Elekes K, Laszlo T, Perkecz A, Pinter E, Sandor Z, Szolcsanyi J, Keszthelyi D, Szabo A, Sandor K, Molnar FT, Szanto Z, Pongracz J, Helyes Zs. Expression of the somatostatin receptor subtype 4 in intact and inflamed pulmonary tissues. Journal of Histochemistry \& Cytochemistry 2009 Dec;57(12):1127-37.

Keszthelyi D, Troost FJ, Masclee AAM. Understanding the role of tryptophan and serotonin metabolism in gastrointestinal function. Neurogastroenterology and Motility 2009 Dec;21(12):1239-49. 
Helyes Zs, Pintér E, Sandor K, Elekes K, Banvolgyi A, Keszthelyi D, Szoke E, Toth D, Sandor Z, Kereskai L, Pozsgai G, Jeremy A, Piers C, Markovics A, Szolcsanyi J. Impaired defense mechanism against inflammation, hyperalgesia and airway hyperreactivity in somatostatin 4 receptor gene-deleted mice. Proceedings of the National Academy of Sciences USA 2009 Aug 4;106(31):13088-93.

Helyes Zs, Pintér E, Németh J, Sándor K, Elekes K, Szabó Á, Pozsgai G, Keszthelyi D, Kereskai L, Engström M, Würster S, Szolcsanyi J. Effects of the Somatostatin Receptor Subtype 4 Selective Agonist J-2156 on Sensory Neuropeptide Release and Inflammatory Reactions in Rodents. British Journal of Pharmacology 2006 Oct;149(4):405-15.

Jakab B, Helyes Zs, Varga A, Bölcskei K, Szabó Á, Sándor K, Elekes K, Börzsei R, Keszthelyi D, Pintér E, Pethő G, Németh J, Szolcsányi J. Examination of the novel TRPV 1 receptor antagonist JYL 1421 (SC0030) in vitro and in vivo in the rat. European Journal of Pharmacology 2005 Jul 4;517(1-2):35-44.

\section{BOOK CHAPTERS}

Keszthelyi D. Health Benefits from Tryptophan Supplementation in Humans: Is there Sufficient Scientific Evidence? in Whitley B and Thornton S (eds.), Tryptophan: Dietary Sources, Functions and Health Benefits. Hauppauge, NY: Nova Science Publishers, 2011 (NLM ID: 101567578, ISBN: 9781621004455$)$

\section{CONFERENCE PROCEEDINGS}

Keszthelyi D. 'All Disease Begins in the Gut': Elucidating Disease Mechanism Related to Intestinal Barrier Dysfunction. Nestlé Purina CAN Summit, The Gastrointestinal Tract in Health and Disease, Lisbon, Portugal, March 2012. 


\subsection{CURRICULUM VITAE}

Daniel Keszthelyi was born in Pécs, Hungary on the $12^{\text {th }}$ February 1984. He attended primary and secondary education in Hungary, the United States and the Netherlands. He obtained his medical degree summa cum laude from the University of Pécs, Hungary in July 2008, having spent one semester at the University of Münster, Germany. He completed clinical rotations in Spain, Austria, Germany, the Netherlands and Sweden. He also served as the president of the European Medical Students' Association in the academic year of 2006/2007. In May 2008, he started working as a PhD fellow at the Division of Gastroenterology-Hepatology, Department of Internal Medicine, Maastricht University Medical Center, under the supervision of Prof. Masclee. The research was funded by the Top Institute Food and Nutrition and was part of the project 'Nutritional modulation of epithelial permeability.' The research performed in this period is presented in the present thesis. During the last year of the $\mathrm{PhD}$ period, he also worked at the laboratory of Dr. Neunlist at the University of Nantes, France. On $1^{\text {st }}$ of September 2012 , he started his training to become a gastroenterologist under the supervision of Prof. Stehouwer (internal medicine) and Prof. Masclee (gastroenterology) at the Maastricht University Medical Center, where he is working currently. Daniel lives together with Rilana and their son, Valentijn. 